LA-10731-T

Thesis

LA- $-10731-T$

DE86 012738

UC-20f

Issued: June 1986

\title{
Far-Infrared Polarimetry/Interferometry for Poloidal Magnetic Field Measurement on ZT-40M
}

Randall Mark Erickson

\section{DISCLAIMER}

\begin{abstract}
This report was prepared as an a
Government. prepared as an account of work

employees, makes any the United States Government nor by an agency of the United States bility for the accuracy, completentess or implied, or assumes any thereof, nor any of their process disclosed, or represents that, or usefulness of any informational liability or responsiits use would not infringe privately owned rights. Refer-
.
\end{abstract}

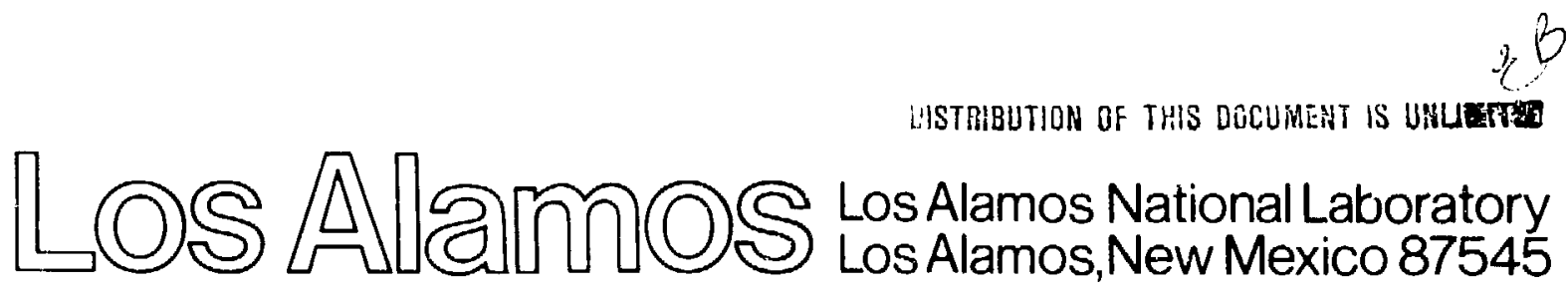




\title{
FAR-INFRARED POLARIMETRY/INTERFEROMETRY FOR POLOIDAL MAGNETIC FIELD MEASUR.EMENT \\ ON 2T-40M
}

by

\author{
RANDALL MARK ERICKSON
}

\begin{abstract}
The measurement of internal magnetic field profiles may be a very important step in the understanding of magnetic confinement physics issues. The measurement of plasma-induced Faraday rotation is one of the more promising internal magnetic field diagnostics. This thesis describes the development of a heterodyne polarimeter/interferometer for internal poloidal magnetic field measurement on ZT40M. Heterodyne technisins were empioyed because of the insensitivity to spurious signal amplitude changes that cause errors in other methods.

Initial problems in polarimetric sensitivity were observed that were ultimately found to be related to discharge-induced motions of the constrained diagnostic access on ZT-40M. Grazing incidence reflections on metallic surfaces of the diagnostic ports causs ? polarization changes that affected the measurement accuracy. Installation of internally threaded sleeves to baffle the reflections eliminated the sensitivity problem, and allowed useful Faraday rotation measurements to be made. Simultaneous polarimetric and interferometric measurements have also been demonstrated.

The ability to assemble a working heterodyne polarimeter/interferometer is no longer in question. The extension of the present system to multichord operation requires increased laser power and efficiency.

Thesis Supervisor: Dr. Franz C. Jahoda Title: CTR-8 Group Leader, Los Alamos National Laboratory

Thesis Supervisor: Dr. Lawrence M. Lidsky

Title: Professor of Nuclear Engineering

Thesis Supervisor: Dr. Daniel R. Cohn

Title: Senior Scientist, Plasma Fusion Center
\end{abstract}




\section{ACKNOWLEDGMENTS}

I would like to take this opportunity to thark my advisers, Dr. Franz C. Jahoda, Professor Lawrence M. Lidsky, and Dr. Daniel R. Cohn, for their guidance in the course of this thesis. Franz in particular offered keen insight at critical points in the thesis research. I thank Dr. Richard Peterson. for his guidance in my undergraduate years and for his role in providing an opportunity to perform research at Los Alamos National Laboratory. I am also grateful for the efforts of all of my professors, for their contributions to my education.

I would like to thank Dr. Peter Forman for his extensive contributions to the development of the FIR system. Without his effort, Faraday rotation measurements on ZT-40M may not have been successful. I also appreciate the initial work of Marvin Bausman, which made this research possible in the scope of a thesis. Discussions with Dr. Paul Weber about the FIR system and on magnetic field measurements in general were also helpful.

I am indebted to Biob Carpenter and Richard Howell for their dedicated technical support, Dr. Ken Klare for his help with data reduction, and Roger Hall for his help with electronics.

I am grateful to the ZT-40M program maragement for the contribution of machine access (as poor as it was) and funding, and for their patience during the diagnostic development. I would also like to thank the ZT-40M team for their interest, and for suppiying sorne of the data that is presented in this thesis. I appreciate the friendship and support of the people I have come to know at Los Alamos.

Finally, I would like to thank my wife, Laura, for typing the text of this thesis, and Dr. Cris W. Barnes for his help in the preparation of this document. 


\section{Contents}

1 Introduction $\quad 10$

1.1 Motivation for Internal Magnetic Field Measurements . . . . . . 13

1.1.1 Interest in Magnetic Field Determination in Tokamak Plas-

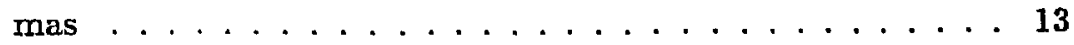

1.1.2 Interest in Magnetic Field Determination in a Reversed Field Pinch ......................

1.2 Possible Techniques for Measuring Internal Magnetic Fields . . . . 17

1.2.1 Passive Techniques for Magnetic Field Measurement . . . . 19

1.2.2 Active Techniques for Magnetic Field Measurement . . . . 21

1.2.3 Relative Merits of Measurement Techniques . . . . . . . 35

2 Theory $\quad 38$

2.1 Propagation of Electromagnetic Waves in a Magnetized Plasma: Appleton's Equation . . . . . . . . . . . . . . . . 39

2.1.1 Ohm's Law from Maxwell's Equations . . . . . . . . . 40

2.1.2 Plasma Resistivity from Fluid Theory . . . . . . . . . . 42

2.1.3 Appleton's Equation . . . . . . . . . . . . . 44

2.2 Limiting Cases of Appleton's Equation . . . . . . . . . . . 45

2.2.1 Leading order clepends only on density . . . . . . . . 45

2.2.2 Propagation along the magnetic field lines: Faraday Rotation 47

2.2.3 Propagation transverse to magnetic field: Cotton-Mouton

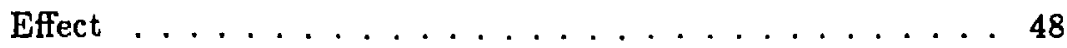

2.3 Inverting Chord-Averaged Measurements $\ldots \ldots \ldots \ldots$

2.3.1 Abel Inversion Methods . . . . . . . . . . . . . 51

2.3.2 Forward Optimization Methods for Inferring the Spatial Profiles . . . . . . . . . . . . . . . 54 
2.4 Error Propagation in the Inversion Process $\ldots \ldots \ldots \ldots$

2.4.1 Techniques used to Study Error Propagation ... . . . 56

2.4.2 Conclusions based on Simple Error Analysis . . . . . . . 58

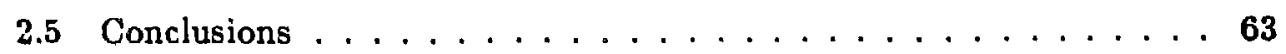

3 EM Wave Probing Tecinniques $\quad 64$

3.1 Techniques for Measurement of Plasma Density through EM Wave Probing of the Plasma Refractive Index . . . . . . . . 64

3.1.1 Measurement of Plasma Density by EM Wave Cut-off . . . 64

3.1.2 Measurement of Plasma Density by Interferometry . . . . . 65

3.1.3 Measurement of Plasma Density by Polarimetry . . . . . 70

3.2 Techniques for Measurement of the Internal Field via $E M$ Wave

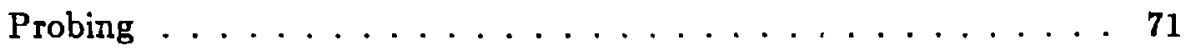

3.2.1 Amplitude Polarimetry . . . . . . . . . 73

3.2.2 Heterodyne Phase Shift Polarimetry . . . . . . . . 76

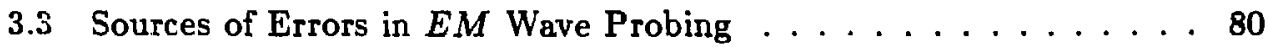

3.3.1 Possible Sources of Errors in a Heterodyne Interferometer . . 80

3.3.2 Sources of Errors in a Heterodyne Polarimeter . . . . . . 82

4 Description of System Employed on ZT-40M 93

4.1 Far-Infrared Laser as a Radiation Source . . . . . . . . . . . . . 94

4.2 Possible Heterodyne Techniques . . . . . . . . . . . . . 99

4.2.1 Electro-optic Rotation of the Pump Polarization . . . . . 100

4.2 .2 Twin FIR Lasers . . . . . . . . . . . . . . . 101

4.2.3 Rotating Grating . . . . . . . . . . . . . 103

4.2.4 Rotating Grating used for Measurements on ZT-40M . . . . 105

4.3 Waveguide Beam Propagation . . . . . . . . . . 112

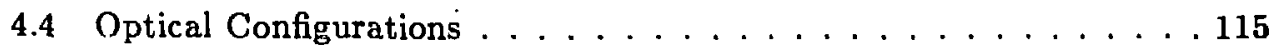

4.4.1 Simple Polarimeter . . . . . . . . . . . . . 115

4.4.2 Simple Interferometer $\ldots \ldots \ldots \ldots \ldots \ldots \ldots$

4.4.3 Simultaneous Polarimetry and Interferometry . . . . . . 117

4.4.4 Multichord Configurations . . . . . . . . . . 121

4.5 Detectors . . . . . . . . . . . . . . . . 124

4.5.1 GaAs Photoconductors . . . . . . . . . . 124

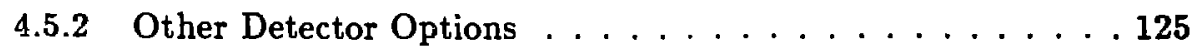


4.6 Signal Processing $\ldots \ldots \ldots \ldots \ldots \ldots \ldots \ldots \ldots \ldots \ldots$

5 Development Required to Obtain Sufficient Sensitivity 129

5.1 Indications of Problems in Polarimetric Sensitivity . . . . . . 132

5.1.1 Anomalous Faraday Rotation Caused by Machine Vibrations 132

5.1.2 Possible Causes of Vibration-Induced Anomaly . . . . . . 133

5.2 Studies of Beam Propagation for the Geometry of ZT-40M . . . 136

5.2.1 Computer Calculations . . . . . . . . . . . 138

5.2.2 Experimental Measurements of Beam Propagation . . . . 138

5.3 Measurement of Tubulation Birefringence . . . . . . . . . 142

5.4 Explanation of Tubulation Birefringence . . . . . . . . . . 143

5.5 Modifications to ZT-40M to Reduce Sensitivity to Vibrations . . . 147

5.5.1 Quartz Inserts on ZT-40M . . . . . . . . . . . . 149

5.5.2 Threaded Inserts on $\mathrm{ZT}-40 \mathrm{M} \ldots \ldots \ldots \ldots \ldots$

6 Measurements on $\mathrm{Z}$ T-40M 154

6.1 Interferometric Measurements . . . . . . . . . . 155

6.1.1 Observation of Refractive-Bending Effects . . . . . . . 156

6.1.2 Time Evolution of Plasma Density on ZT-40M . . . . . 159

6.1.3 Particular Measurements by the Far-Infrared Interferometer . 161

6.2 Polarimetric Measurements . . . . . . . . . . . . 166

6.3 Simultaneous Polarimetry and Interferometry $\ldots \ldots \ldots \ldots \ldots$

7 Summary, Conclusions, and Recommended Future Work 177

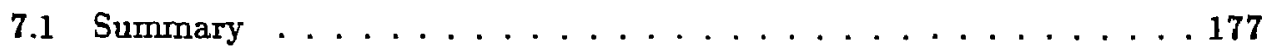

7.2 Conclusions and Recommendations $\ldots \ldots \ldots \ldots \ldots \ldots$

A Definitions in Toroidal Coordinates 186

B Mathematical Methods for Calculating Polarization Effects 188

B.1 The Crystal Optic Analogy of a Magnetized Plasma . . . . . 188

B.2 Polarization Description and Evolution: The Poincaré Sphere . . . 190

B.3 Jones Matrix Calculus for EMr Wave Propagation . . . . . . . 195

$\begin{array}{ll}\text { C Description of Far-Infrared Optical Components } & 198\end{array}$ 


\section{List of Figures}

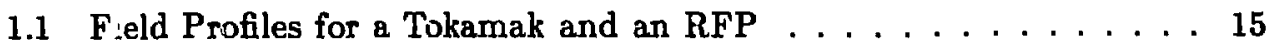

1.2 Zeeman Splitting of the Lithium $2 p-2 s$ Transition . . . . . . . 30

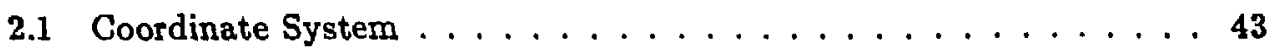

2.2 Poloidal Cross-Section Showing Geometry for Abel Inversion . . . 52

2.3 Two Possible Profiles for Minimum Norrn Criterion . . . . . . . . . 55

2.4 Density and Field Profiles for $250 \mathrm{kA}$ Plasma, $3 \mathrm{mrad}$ Resolution . . 60

2.5 Density and Field Profiles for $120 \mathrm{kA}$ Plasma, 3 mrad Resolution . . 61

2.6 Poloidal Field Profile for $120 \mathrm{kA}$ Plasma, 0.5 mrad Resolution . . 62

3.1 Michelson Configuration . . . . . . . . . . . . 68

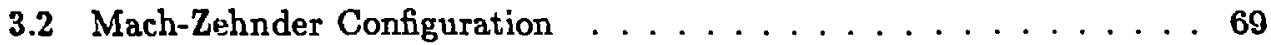

3.3 Simple Polarimeter . . . . . . . . . . . . . . . . 74

3.4 Soltwiscin Configuration $\ldots \ldots \ldots \ldots \ldots \ldots \ldots \ldots$

3.5 Ferrite Rotator Configuration . . . . . . . . . . . . . 77

3.6 Heterodyne Polarimeter . . . . . . . . . . . . . . 79

3.7 Component System Used for Error Analysis . . . . . . . . . . . 82

3.8 Example of Faraday Rotation Enhancement . . . . . . . . . . 90

4.1 Schematic of FIR Laser $\ldots \ldots \ldots \ldots \ldots$

4.2 Heterodyning by Rotating Pump Polarization . . . . . . . . . 101

4.3 Heterodyning by Using Frequency-Offset Lasers . . . . . . . . . . . 102

4.4 Heterodyning by Using Rotating Grating . . . . . . . . . . . 103

4.5 Grating Geometry . . . . . . . . . . . . . . . 104

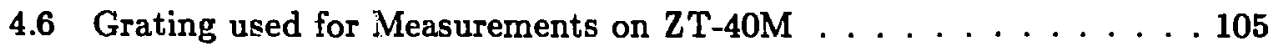

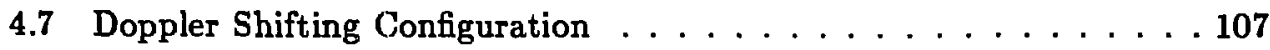

4.8 Phase of a Typical Reference Signal . . . . . . . . . . . . . . 109

4.9 Density Information Superimposed on Baseline Drift . . . . . . . 109 
4.10 Grating Ghosts and Mechanical Vibrations . . . . . . . . . 111

4.11 Baseline with Four-Signal Technique . . . . . . . . . . . 112

4.12 Heterodyne Polarimeter on ZT-40M . . . . . . . . . . 116

4.13 2T-40M Interferometer . . . . . . . . . . . . . 118

4.14 Simultaneous Configuration . . . . . . . . . . . . 119

4.15 Revised Simultaneous Configuration . . . . . . . . . . . . . 122

4.16 Two-Chord Simultaneous Configuration . . . . . . . . . . . 123

4.17 Preamplifier Circuit for GaAs Photoconductor . . . . . . . . . . 124

4.18 Method for Calculating Carrier-Signal Phase . . . . . . . . 128

5.1 Polarimetric Measurements Indicating Sensitivity Problems . . . . . 134

5.2 Theoretical Beam Profiles for Geometry of ZT-40M . . . . . . . . . 139

5.3 Set-up for Beam Propagation Measurements . . . . . . . . . . . . . 140

5.4 Measured Intensity Profiles for Geometry of ZT-40M . . . . . . . . 141

5.5 Crossed Polarizer Test Set-up . . . . . . . . . . . . . . . 142

5.6 Polarization Azirnuth Rotation for Bare Metal, Quartz, and Threaded Tubulations ....................... 144

5.7 Reflectivities and Phase Difference for $s$ and $p$ Polarizations for Grazing Angles of Incidence . . . . . . . . . . . . . . . . . 146

5.8 Polarization Rotation vs. Incident Azimuth . . . . . . . . . 148

5.9 Quartz Inserts after Removal from ZT-40M . . . . . . . . . . . 150

5.10 Profile of Metallic Deposition on Quartz . . . . . . . . . . . . 151

5.11 Polarimetric Phase for ZT-40M Discharges with Bare Tubulations, Bypassing Torus, and with Threaded Inserts .......... 153

6.1 Interferometric Measurement with Two-Signal Technique . . . . . 157

6.2 Example of Interferometric Measurement in Simultaneous Configu-

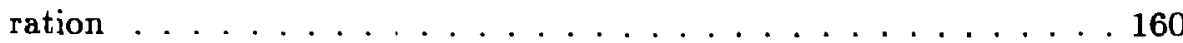

6.3 Central- and Edge-chord-averaøed Densities . . . . . . . . . 162

6.4 Examples of Density Sawtooth Oscillations . . . . . . . . . . . . 164

6.5 Density of Ramped (70 $k A$ to $170 k A$ ) Discharge Compared to Standard $170 k A$ Discharge . . . . . . . . . . . . . . . . 165

6.6 Carrier Signal Measured by the Polarimeter . . . . . . . . . . 166

6.7 Toroidal Current and Faraday Rotation for a Design-Mode Dis-

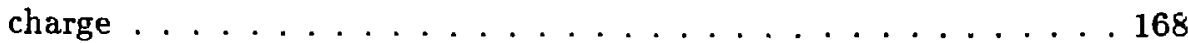


6.8 Current, Density, and Faraday Rotation for $300 k A$ Discharges . . 170

6.9 Faraday Rotation Normalized by Density and $I_{\phi} \ldots \ldots \ldots \ldots 1$

6.10 Simultaneous Measurement of Density and Faraday Rotation . . . 174

A.1 Coordinate System for Toroidal Geometry . . . . . . . . . . 186

B.1 Polarization Defined in Terms of Ellipticity and Azimuth . . . . 190

B.2 Description of Polarization on Poincaré Sphere . . . . . . . . 191

B.3 Operations Required to Determine Polarization Evolution . . . . . 193

B.4 Graphical Determination of Axis of Rotation for Elliptical Birefrin-

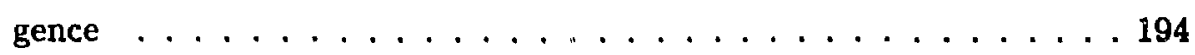




\section{List of Tables}

1.1 Relative Merits of Measurement Techniques . . . . . . . . . . 36

4.1 Laser Gases . . . . . . . . . . . . . . . . . . . 98

6.1 Calculated Faraday Rotation Levels for Various Profiles . . . . . 175 


\section{Chapter 1}

\section{Introduction}

Allowing that magnetic confinement of plasmas has been the leading approach to controlled fusion over the past twenty-five years, it is somewhat surprising that measurement techniques for local magnetic fields are not by now, well establighed and routine. Several methods, involving very different physical principles, have been attempted, with some measure of success in recent years; but it would seen that the quest for a simple, reliable and accurate technique is still very actively being pursued. [1]

This thesis research has been devoted to the pursuit of one such internal magnetic field measurement technique. The particular objective of this effort was to develop a capability for measuring the poloidal magnetic field $\left(B_{\theta}(r)\right)$ in ZT-40M - a Reversed-Field Pinch (RFP) plasma - by Faraday rotation (see Appendix A.1 for a definition of toroidal coordinates). Similar measurements have been developed for measurements on Tokamak plasmas, where a related quantity, the toroidal current distribution, is sought. The real interest in RFP physics is a knowledge of both magnetic field components $-B_{\theta}(r)$ is only part of the problem. (The methods adapted for measurements on ZT-40M could be used tor toroidal field measurements if the appropriate diagnostic access were available [2].)

There are many diagnostic techniques that have been considered and developed to various stages. None of the techniques have proven to be ideal in all respects. What is sought is a measurement system that is simple and reliable, accurate and unambiguously interpreted, and able to provide single-shot profile information - 
not only for the present laboratory plasmas, but for reactor-grade plasmas of the future.

For the purposes at hand, it is important to recognize that many of the $B_{\theta}(r)$ measurement techniques (that have been developed to the point that measurements on relevant plasmas are possible) are only applicable to tokamak plasmas. They require a priori knowledge of the $B_{\phi}(r)$ profile, making them unsuitable for measurements on other plassas (such as an RFP plasma). Some techniques have limitations in the ability to make measurements in a reactor-grade plasma, although measurements of present laboratory plasmas are possible. Others are difficult to implement on the present plasmas, but become much easier as reactor conditions are approached.

The various magnetic field measurement techniques are surveyed in the following sections. Faraday rotation is clearly one of the leading candidates for making magnetic field measurements on RFP plasmas. Briefly, the important featises of Faraday rotation measurements are:

- the ability to make single-shot determinations of the magnetic field profile,

- the ability to make such measurements on a wide variety of plasmas,

- the requirement of diagnostic access that is standard on most machines, and

- its close relationship in design and operation to interferometry - a well developed and widely used diagnostic technique.

Faraday rotation measurements are rather difficult on most machines today, because of the small rotations induced by present laboratory plasmas. However, the measurements will become rnuch essier as reactor conditions are approached.

Because Faraday 1 otation results from the interaction between and electromagnetie $(E M)$ probe vesm and the plasmas, the theory of $E M$ wave propagation is developed in Chapter 2, with the help of appendices. Density effects on $E M$ wave propagailo : are also considered because density measurements need to be made in conjunction with Faraday rotation measurements in order to extract the magnetic field information. Although the measurements can be made independently, there are schemes that allow both density and Faraday rotation measurements to be nade simultaneously. 
Various $E M$ wave probing techniques will be discussed in Chapter 3. There are a nurnher of techniques that can be used for making Faraday rotation measurements. Most techniques sense a change in signal amplitude generated by Faraday rotation. The implest example of such a technique is monitoring the changes in transmission through a linear polarizer that result from the Faraday rotation induced by the plasma. This elass of measurement technique (from this point on to be referred to as amplitude polarimetric technique) suffers from uncertainties caused by other physical effects that can alter the amplitude of the signal. The method for measuring Faraday rotation on ZT-40M is an alternative approach, one that is relatively insensitive to spurious amplitude changes. The concept is closely related to heterodyne interferometry, where the density alters the phase of a carrier signal, and the density information can be recovered even if large signal amplitude changes may occur. In heterodyne phase-shift polarimetry, the Faraday rotation alters the phase of a carrier signul, and by analyzing the time signature of the carrier signal, the magnetic field determination is independent of spurious amplitude changes.

The equipment that was used for measurements on $2 \mathrm{~T}-40 \mathrm{M}$ is described in Chapter 4. The system developinent described in Chapter 4 pertains to the implimentation of Faraday rotation measurements on any device. There was additional development required to obtain sufficient polarimetric sensitivity that was specific to the application to ZT-40M. An account of this work is relegated to Chapter 5. The problems were caused by the constrained access on ZT-40M, and may have relevance to applications of Faraday rotation where similar access characteristics are present.

One of the primary results of the thesis research is the demonstration that Faraday rotation measurements are indeed possible on RFP plasmas, although the problems encountered were found to be much more severe than similar experience in tokamak applications. Very interesting observations result just from the inspection of a polarimetric carrier signal, where spurious amplitude changes preclude the use of amplitude techniques for Faraday rotation measurements on ZT.40M. Some interesting conclusions can be drawn from the single-chord measurements on ZT-40M; how ver, a common theme of Chapter 6 is the need of multichord measurements to accurately understand many of the effects. As a result of this thesis research, there is very little doubt that a multi-chord system could be assembled 
and successfully operated. The extension of the techniques used for single-chord measurements to multi-chord operation is discussed in Chapter 7.

\subsection{Motivation for Internal Magnetic Field Mea- surements}

Although simple and reliable techniques for making routine internal magnetic field measurements are not yet available, it is commonly believed that such measurements would be extremely useful in the enhancement of urderstanding of many important processes in plasma confinement. The lack of detailed measurements has prompted various siudies on how to make inferences about internal fields from external magnetic measurements (e.g.,(3)), or from very minimal internal measurements[4].

There are a number of important issues that could be studied if internal magnetic field measurements were available. Some of the issues that are common to all confinement schemes are the magrietic field profile formation and sustainment, and the equilibrium, stability, and termination of the plasma. There are other measurements that may address these issues to some degree, and detailed theories have been formulated to explain observations. Magnetic field measurements could serve to augment or refute the present understanding of these and other issues.

\subsubsection{Interest in Magnetic Field Determination in Toka- mak Plasmas}

For a tokamak plasma, some of the information that could be obtained from an internal magnetic field measurements was listed by Équipe TFR in an excellent review of tokamak plasma diagnostics[5]. Information about the internal magnetic field in a tokamak would lead to a better understanding of skin effects, plasma stability, power balance, and runaway electrons. Skin effects are an important issue in a tokamak because classical magnetic field diffusion theory predicts that skin currents induced in the plasma formation phase do not have time to diffuse to the center[6]. Thomson scattering temperature measurements contradict this theory, indicating that the conductivity is peaked at the center on a relatively short time scale. Internal magnetic measurements could help in the understanding of this anomalous radial current transport observed in experiments. 
Basic MHD stability deperids on the magnetic field profile in the plasma. Although tokamak stability is relatively well understood, internal magnetic measurements could help test the theory. With the aid of magnetic measurements, effects important in determining the stability could be more clearly established, possibly resulting in our extension of the present stability regime.

Although the global power balance can be determincd by measuring the total toroidal current, knowledge of the current density profile is necessary to calculate the local power balance. It is required to determine the local ohmic input power.

Information about runaway electrons can be obtained from internal magnetic field measurements as long as there is independent information about the current generated by the buik electrons alone. This independent measurement could be made by measuring the shift in the electron velocity distribution via Thomson scattering, which will be discussed in Section 1.2. Measurements would need to be extremely accurate because a very small percentage of the current is generated by runaway electrons [7]. Runaway electrons were of interest in the early development of tokamaks because of severe wall damage that was caused by these electrons [7]. In recent years, a lower energy class of electrons, "slideaway" electrons, are the result of current drive schemes in tokamaks, where the tail of the electron velocity distribution is enhanced, and the stabilizing plasma current is generated by a minority of the electrons, rather than the bulk electrons.

In a tokamak plasma, the characteristically low $\beta^{1}$ is an advantage for internal magnetic field determination. This is becuuse the internal magnetic field does not deviate significantly from the vacuum magnetic field. The toroida! field is determined with adequate precision by the vacuum relation $B_{\phi}(R) \propto 1 ; R$, where $R$ is the major radial coordinate (see Appendix A. for a description of toridal coordinates). Internal magnetic field measurements on a tokamak are, therefore, directed toward determining the poloidal magnetic field profile, or equivalently, the toroidal current density distribution. Since the tokamak toroidal field is known, measurement techniques that indicate the magnetic field direction with adequate precision can directly lead to a determination of the poloidal field strength.

Although the low $\beta$ of the tokamak plasmis facilitates internal magnetic field measurements, it makes the tokamak less attractive as a fusion reactor. There are minimum requirements of plasma density and temperature for reactor operation,

\footnotetext{
${ }^{1} \beta \equiv(n k T) /\left(B^{2} / 2 \mu_{0}\right)$ is the ratio of the plasma pressure to the magnetic pressure.
} 

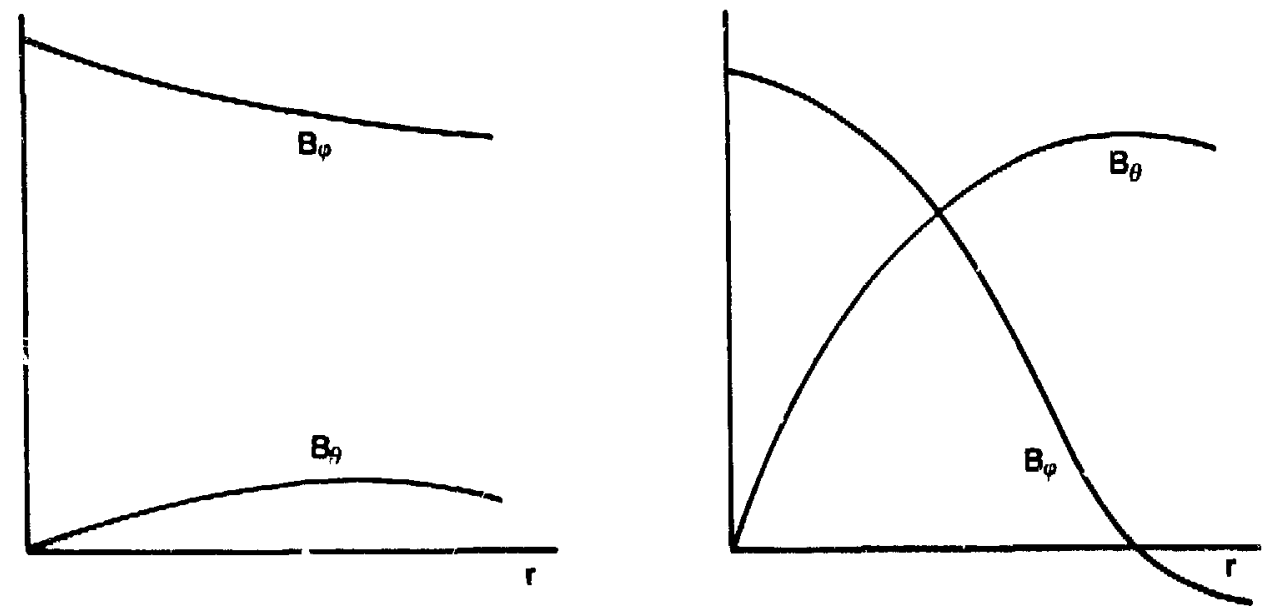

Figure 1.1: Field Profiles for a Tokamak and an RFP

and the parameter $\beta$ indicates the magnetic field strength necessary to support the plasma. Generating magnetic fields is a significant cost in a fusion reactor, both in magnet coil power consumption and in mechanical structure to support the forces on the coils. Therefore, low $\beta$ results in higher costs - an important concern for an economically marginal energy technology.

\subsubsection{Interest in Magnetic Field Determination in a Re- versed Field Pinch}

Many of the issues important to tokamak confinement are also important in a reversed-field pinch (RFP) [8]. However, in an RFP, there is a greater need for internal magnetic field measurement. Unlike a tokamak, there is as much uncertainty in the toroidal magnetic field as there is in the poloidal field. The RFP is a high $\beta$ device, which causes the internal fields to deviate extensively from vacuum fields. Theoretical magnetic field profiles for the RFP are compared to those of a tokamak in Figure 1.1. Note that the vertical scales are quite different (the $B_{\theta}$ values are comparable if the plasmas have the same toroidal current). The primary differences between the fields of the two confinement schemes are the much higher torsidal field in the tokamak, and the distinctive toroidal field profile of the RFP. The vacuum 
toroidal field on an RFP must also follow the relation $B_{\phi}(R) \propto 1 / R$, but the field in the presence of the plasma is grossly different. The actual mechanism for generating and sustaining such a reversed field configuration is not completely understood. Theoretical models of possible mechanisms have been proposed, (e.g.,[9][10]), however, detailed internal field measurements are required to substantiate the theory, and verify that the actual profiles do not deviate from these theoretical profiles. In addition to the lack of understanding of the magnetic field profile generation and sustainment, Gerwin [4] has outlined other important issues in RFP confinement physics that would benefit from a knowledge of the magnetic field profiles. These issues are reviewed in the following sections.

\section{Stability of RFP Plasmas}

Magnetic field profiles enter into the MHD stability of the RFP, where stability is achieved, in part, by the strong shear of the magnetic field. Measurement of the field profiles could be checked against ideal and resistive MHD stability criteria, although a measurement of the plasma pressure profile is required to complete the stability analysis. The magnetic field profiles could also be analyzed for susceptibility to micro-instabilities, and magnetic fluctuations could be correlated to fluctuations measured in other plasma parameters to give information on modes that might only be marginally stable.

\section{Transport Code Physics}

Transport codes are used extensively to explore transport phenomena in RFP plasmas [11][12][13]. Measured magnetic field profiles, along with an independent knowledge of the pressure profile, could be used for initial conditions for the codes; or used as running comparisons with the code results. Gerwin[4] considers that this sort of tandem investigation could be very valuable in providing information about the nature of effective transport coefficients.

\section{Measurement of other Plasma Parameters}

Knowledge of the magnetic field profiles, and therefore the current density profiles, can provide a measurement of the plasma resistivity on axis. Values of the resistivity on axis inferred from magnetic field measurements could be compared 
to the Spitzer resistivity on axis inferred from Thoinson scattering measurements, perhaps providing more information about the anomulous resistivity observed in experiments (this assumes the plasma is in steady state). Measurement of the internal magnetic field profilss could also provide indirect indications of other parameters. For example, the measured current profile could be used to derive a "conductivity temperature" profile that could be compared to other measurements that provide some information about the plasma temperature profile.

Th.e power local power balance in an RFP plasma requires more information than a tokamak plasma. Both poloidal and toroidal currents contribute to the ohmic input power. Therefore measurements of both internal field profiles are necessary to completely determine the local power balance.

Despite the lack of information about the internal magnetic field, the performance of magnetic confinement devices has advanced significantly in the past twenty years. Some of the improvements have resulted from improving the magnetic topology, in the form of alteration of the magnetic flux surfaces ard reduction of magnetic field errors. The advent of internal magnetic field diagnostics will undoubtedly contribute to future improvements in magnetically confined plasmas. The following section is devoted to a review of the various internal magnetic field measurement techniques that have been proposed.

\subsection{Possible Techniques for Measuring Internal Magnetic Fields}

Much of the work in diagnostic developinent has been performed on tokamak plasmas, so it should not come as a big surprise when it is found that some techniques are specific to tokamak applications. This is especially true for magnetic field measurement techniques, where unique characteristics of the tokamak field profiles allow the use of some techniques that would not be applicable to other plasmas. The toroidal field is monotonically decreasing with major radius, and is known with adequate precision due to its similarity to the vacuum toroidal field. Therefore, for example, techniques that measure the field-line direction provide the final piece of information about tokamak magnetic fields. The characteristics of the tokamak toroidal field profile also facilitate certain techniques, as will be discussed below. 
The standard method for making magnetic field measurements is to use some sort of probe[14]. An example of a magnetic field probe is a simple pick-up loop, where currents are generated by changing magnetic fields. Such probes are useful for pulsed magnetic fieids. For the measurement of DC magnetic fields, Hall effect probes are extensively used. These probes sense the magnetic field directly rather than measuring the rate of change of the field.

Although probes can provide extensive information about plasma magnetic fields [15], they cannot be used in plasmas of thermonuclear iaterest. Severe problems are found in probe durability in the harsh environment of the plasma, where common materials cannot withstand the heat loads. Even if this were not a problem, a probe is a perturbing element in the plasma, and alters the characteristics of the plasma that is being probed. In addition to the introduction of impurities in the plasma due to probe erosion, the probe can actually introduce distorticns to the magnetic field[14]. Thus for the measurement of the magnetic neld internal to the plasma, the common measurement techniques cannot be applied. Indirect and complicated techniques are required.

Theie are many indirect measurement techniques that have been proposed, based on plasma phenomena that depend in some way on the magnetic field. In most cases, the measurement of the external magnetic field will be ignored; however, such measuremerts can add to the other information obtained and contribute to the overall understanding of the plasma magnetics. The indirect methods for measuring the internal magnetic field fall into two categories: passive measurements and active measurements. The passive methods involve the measurement of chararteristics of the various types of plasma emissions, which exist whether or not any measurements are being made. Active methods involve the injection of non-perturbing "probe" (some form of wave or particle beam), and measuring the characteristics of the probe that are altered by the plasma. The active techniques are more complicated than the passive techniques, because the waves or particles need to be generated as well as detected. However, pasaive techniques often suffer from weak plasma emissions and sometimes produce ambiguous or underdetermined information, and in these cases, active measurements are required.

The choices between the many options for measuring the magnetic field are highly machine-dependent. Some methods require detailed independent knowledge of one of the magnetic field components, and therefore may only be useful on a low 
$\beta$ tokamak where the toroidal magnetic field is known. Other methods, which may be applicable to any plasma, require diagnostic access that may be impossible to obtain for certain confinement schemes. Some methods also may not be able to be expanded to allow measurement of magnetic profiles in a single plasma discharge.

In spite of the many options available for making measurements of the internal magnetic field, none of the options clearly stand out as having an advantage in simplicity, censitivity, and ease of interpretation. Most options are difficult to implement and/or difficult to unambiguously interpret, and the internal magnetic field is probably the most poorly diagnosed plasma characteristic at this point in time. The following sections outline the passive and active measurement techniques that have been proposed, the status of the development of the measurements, and the difficu'ities encountered or foreseen in the diagnostic development and imnlementation. it is not intended to be a complete treatment of these measurement techniques. The basic concepts and prospects of the various ideas will be reviewed; and the interested reader will be referred to the work of oihers for more details. (An excellent review of magnetic field diagnostics is presented by Peacock [1].)

\subsubsection{Passive Techniques for Magnetic Field Measurement}

\section{External Magnetics, MHD Activity}

The most common passive internal magnetic field estimate combines information obtained from the external magnetic field with measurements of internal plasma parameters other than the magnetic field. References describing the measurement technique in more detail are [16] and [17]. The information required for determining the current density distribution includes the pressure profile (from Thomson scattering), the $q=1$ surface (from soft $x$-ray sawteeth), the total current (from a Rogowski coil), the edge poloidal field (from a loop probe array), and the $Z_{\text {eff }}$ profile (by measurement of bremsstrahlung continuum, which is $\propto\left(Z^{2} n^{2} / \sqrt{T}\right) e^{-h \nu / T}$ ). This information is used to give a self-consistent solution to the Grad-Shafranov equation for the current density:

$$
j(R, \psi)=R \frac{d p}{d \psi}+R B_{\phi} \frac{\left(R B_{\phi}\right)^{\prime}}{\mu_{o} R},
$$

where $R$ is the major radial coordinate, $\psi$ is a flux coordinate, $B_{\phi}$ is the toroidal field, $p$ is the pressure and the prime denotes the partial derivative with respect 
to $\psi$. This technique is universally employed in tokamaks; however, it is only applicable when a $q$ value can be determined and the other measurements are available.

\section{Cyclotron Emission Polarimetry}

The second passive cyclotron s siation emitted from the plasma. The polarization of the radiation depends on the magnetic field direction at the point of origin. This technique would be particularly useful for a tokamak plasma, because the cyclotron frequency is determined primarily by the toroidal field strength, which is known to be $\propto 1 / R$. Therefore, the measurement of polarization as a function of frequency yield's a determination of the magnetic field line pitch as i function of major radius, from which the poloidal field component can be extracted (because the toroidal field strength is known). This method is not applicable to plasmas that don't have these special characteristics.

This technique was attempted on the TFR tokamak [18]. Experimental diffculties were encountered that were attributed to polarization scrambling by wall reflections and plasma turbulence. It is also possible that polarization changes resulting from propagation through the plasma might have made the polarization measurement difficult. ${ }^{2}$ Additional problems were seen with absorption of the emission by the plasma, and the competing effect of plasma frequency emission by a low density hot electron component of the plasma. As a result this attempted measurement of the internal magnetic field by cyclotron emission polarimetry was unsuccessful.

\section{Fusion Product Emission}

The ability to determine poloidal magnetic field profiles by measuring fusion product emission was studied by Heidbrink on the PLT tokamak [20]. Fusion products leave the plasma on trajectories that depend an the emission profile and magnetic field. For measurement of the emission profile, detectors are placed at positions that minimize sensitivity to magnetic field effects. For poloidal field measurements,

\footnotetext{
${ }^{2}$ Such effects are discussed in Reference [19]. Due to polarization changes in propagation, the emitted polarization depends on the edge magnetic field rather than the field at the point of origin.
} 
detectors are placed in positions where the fusion product emission depends sensitively on the magnetic field.

Although measurements on PLT indicated when the current distribution was more peaked or more broad than in standard operating conditions, there are problems in uniquely determining a poloidal field profile with this technique. This is especially true when there are uncertainties in the toroidal field profile - when the fusion product trajectories are not well known. However, the qualitative information that can be gained with relatively low complexity makes this technique attractive for plasmas of appropriate parameters for fusion product generation.

\subsubsection{Active Techniques for Magnetic Field Measurement}

\section{Low Freciuency Wave Probes}

One class of active magnetic field diagnostics involves the injection of various lowfrequency waves into the plasma. The resulting plasma-wave interactions can be monitored, which can lead to some level of understanding about the characteristics of the plasma. There are some waves that can interact with the plasma in ways that depend on the magnetic field.

Upper Hybrid Layer Harmonic Generation One such technique is described by Cano [21], where the poloidal field can be determined when the toroidal field is dominant and known, and the upper hybrid layer is accessible. In a tokamak, the method involves injecting a wave into the plasma from the inside major radius, with the frequency chosen to resonate with the upper hybrid frequency at some position in the plasma. The position of the upper hybrid resonance layer is determined by measuring the density profile (via Thomson scattering), and determining the position where the density and magnetic field parameters at a given position solve the resonance condition, $\omega=\omega_{U H}=\left(\omega_{p e}^{2}+\Omega^{2}\right)^{1 / 2}$, where $\omega_{p e}$ is the plasma frequency, $\Omega$ is the cyclotron frequency, and $\omega_{U H}$ is defined to be the upper hybrid frequency. At the resonant layer, the second harmonic is generated, and the polarization of the second harmonic is determined by the local field direction. Measurement of the polarization is then a determination of the pitch angle of the magnetic field at the resonance point, and the poloidal component can be found if the toroidal field is a known quantity. Independent knowledge of the toroidal field strength is required 
both for the determination of the spatial location of the resonant interaction and for the poloidal field determination from the pitch angle. Therefore, this technique is only applicabie to a tokamak plasma.

There is another reason why this method is only applicable to tokamaks. The accessibility oi the upper hybrid layer in a tokamak requires thsi the wave be injected from the inboard side of the plasma, approaching the resonant layer from the high field side. If the wave is injected from the outboard, or low field side, the wave is cut off (or reflected) before the resonant layer is reached. The fiell profiles of reversed field pinch are such that a wave injected from any location is cut off, and the upper hybrid resonance is inaccessible unless optical mixing techniques are employed (see the section on optical mixing below).

In many respects, this technique has many similarities to the cyclotron emissiun measurement discussed eariier, except in this case there is no reliance on plasma emission, which may not always be ideally suited for making the measurements of interest. This is usually due to emission levels that are lower than one would like. This illustrates clearly the virtue of an active technique over a passive technique, and in this case it has contributed to a modest success of upper hybrid layer harmonic generation as a poloidal field diagnostic. Cano [21] presents results of such measurements in the ST tokamak, where the poloidal field is found roughly consistent with the plasma current profile exhibiting a $T_{c}^{3 / 2}$ dependence (i.e., consistent with Spitzer resistivity). In order to obtain single shot field profiles by this technique, the wave probe frequency would need to be swept on a timescale that is much faster than the pulse length of the discharge.

Magneto-Acoustic Wave Propagation The concept of measuring the magnetic field profile by measuring the propagation velocity of an excited magnetoacoustic (compressional Alfvèn) wave is presented by Howell and Cayton [22). The idea is based on mugneto-acoustic waves that propagate normal to flux surfaces at a speed that is determined by the magnetic field, temperature, and density profiles of the plasma. With independent knowledge of the density and temperature profiles (from Thomson scattering), the magnetic field can be determined by measuring the wave propagation velocity. This could be done in a "time of flight" mode, where the waves are excited by external loops at one location and detected elsewhere.

This technique has the drawback of requiring almost the entire spectrum of 
the response in oider to adequately determine the magnetic field. No experiments have been attempted for measuring the field in this way. The inverse problem (measuring $B$ and determining the plasma pressure from the wave propagation) is much more sel, ; itive[23].

Alfvèn Wave Mode Conversion Another method for measuring $B_{\theta}(r)$ with low frequency waves was proposed by Mahajan [24]. A low frequency wave is launched from the plasma edge and propagated to the Alfvèn resonance layer where mode cuaversion to the kinetic shear Alfvèn wave takes place. The resonant frequency is given by:

$$
\omega=k_{\|} V_{A}=\frac{\left(\frac{m}{q}-\ell\right)}{R} \frac{B}{\left(\mu_{o} \rho\right)^{1 / 2}},
$$

where $m$ and $\ell$ are the poloidal and toroida! mode numbers, and $q$ is the safety factor (see Appendix A). Sizable density fluctuations result and the position of the resonance layer can be found by a $\mathrm{CO}_{2}$ laser scattering experiment. The poloidal field at that position $\left(r_{s}\right)$ is related to the resonant frequency by the following expression for the axisymmetric case $(\ell=o)$ :

$$
B_{\theta}\left(r_{s}\right)=\frac{\left(\mu_{o} m_{i}\right)^{1 / 2}}{m} \omega r_{s} n_{o}^{1 / 2}\left(r_{s}\right) .
$$

Such a diagnostic has been implemented on the tokamak Prete:.t; however, no results have been published. The problem with the technique is that the mode purity of the launched wave must be very good in order to adequately localize the density fluctua:ions. Also, the plasma must have an Alfvèn resonance that is spatially varying, and many probe frequencies need to be employed in order to get the full field profiles.

\section{Heavy Particle Beam Probes}

Particle beam probes can be used to obtain information about the internal magnetic field of a plasma. The primary effect that allows the field to be measured is the deflection of a charged particle trajectory due to the Lorentz force.

Heavy Ion Beam Probes The principle for magnetic field measurements using heavy ion beam probes is discussed by Jobes [25]. If the beam has sufficient energy, the Larmur radius of the particle will exceed the plasma radius, and the particle 
will pass through the plasma. The location of the deflected particles after passing through the plasma can be compared to the location of the particles after passing through the vacuum fields alone. This can give some information about the internal magnetic field; however, problerns in uniquely determining the internal field result from the many field profiles that could be consistent with the measured particle trajectories. The problem is so severely underdetermined that no unique solution can be found. Further uncertainties are caused by the possibilities of charge alteration within the plasma. Multiple processes can restore the original charge, but the trajectory may be altered to the point that erroneous information can be produced by the measurement. An additional problem is generated by the requirement of specialized diagnostic access that is generally not available. All of these factors have contributed to the lack of success of this technique.

Heavy Neutral Beam Probes A similar technique is based on Larmor trajectory to infer the magnetic nield, but uses a probe beam that is initially neutral [26]. The advantage of this method over that of the previous section is the ease of injecting the probe into the plasma. As neutrals, the particle trajectories are not bent by the external magnetic field or the plasma field until the particles are collisionally ionized. The plasma potential can also be measured with these particles, by comparing the final particle energy with the initial energy, which will differ by $q \Phi$, where $q$ is the charge of the particle and $\Phi$ is the potential of the plasma at the fuint of ionization. (The plasma potential can also be measured by the heavy ion beam probe discussed earlier if a second ionization takes place in the plasma.)

Again, even if the ionization location is accurately known, (in practice it is only approximate), the problem with this method is that the magnetic field effect is integrated along the path of the particle, and details of the magnetic field profile are difficult, if not impossible, to unfold. Added uncertainty is intrcduced by the possibility of multiple ionization processes within the plasma. Masamune, et ai.,[26] claimed that one should at least be able to distinguish between a flat and a parabolic current profile with this method. Even if more detailed measurements were possible with this method, it would not be useful for reactor-grade plasmas because of the beam energy required to have large Larmor orbits. 


\section{Light Particle Beam Probes}

Light particle beam probes can aiso be used to measure the magnetic field. Neutral beams are required in order to inject the particles into the plasma, because the Larmor radius of a light ion is too small for the ion to get through the magnetic field external to the plasma. It is this small Larmor radius in the plasma that gives light ion beams an advantage over hesvy particle beams in the ability to make local measurements of the magnetic field. The neutral particles are ionized or charge exchanged by collisions in the piasma, altered in some way by the magnetic field, and, to be detected, must neutralize by charge-exchange to escape from the plasma. Two measurement techniques have been proposed, although only one has been demonstrated. One technique requires tangential beam injection and the other requires perpendicular beam injection.

Tangential Injection - Guiding Center Drift Measurement A neutral beam can be tangentially injected into the plasma, where collisional ionization converts a fraction of the beam into ionized particles with mostly parallel energy. In a uniform magnetic field, the ions gyrate along the magnetic field lines. However, due to magnetic field gradients and field line curvature, the gyrating ions experience a guiding center drift. In a tokamak plasma the maximum deviation or displacement from a. Hux surface experienced by a circulating ion (as opposed to a "banana-trapped" ion) is given by:

$$
\Delta \simeq q_{s} v_{\|} / \Omega,
$$

where $q_{s}$ is the safety factor, $v_{\|}$is the parallel velocity of the particle, and .2 is the cyclotron frequency. The toroidal position where this maximum occurs can be roughly calculated, and a charge-exchange detector array can look for probe ions that charge-exchange and escape the plasma at this location.

A description of such a system, along with measurements that were made on the ACT tokamak are presented by Goldston [27]. In principle, the measurament could be made with a monoenergetic, single species beam by estimating the minor radial ionization position and comparing it to the minor radius where the charge-exchange neutral originated. In practice, it is found that, a multi-species, multi-energy beam aids in the guiding center displacement determination. The neutral beam used for meas: rements on ACT was composed of $D^{\circ}$ at $E_{o}, D^{\circ}$ at $E_{o} / 2, D^{\circ}$ at $E_{o} / 3, D_{2}^{\circ}$ 
at $E_{o}$, and $D_{2}^{\circ}$ at $2 E_{o} / 3$, with $E_{o}=30 \mathrm{keV} .^{3}$ Each of the components of the bean experiences a different guiding center drift, and if the difference in guiding center between the components is measured, the $q$, profile can be determined. For a tokamak where $B_{\phi}$ is known, this corresponds to a measurements of $B_{\theta}(r)$. On the ACT experiment, the beam was chopped to distinguish the probe particles from background particles, or particles that may have made several circuits around the torus. The plasma was translated in major radius on ACT in order to get spatial field profiles; however, this is not generally possible, and obtaining profiles with this technique is difficult unless many beams are used and many toroidal access ports to the plasma are available.

Perpendicular Injection - Pitch Angle Measurement If a neutral beam is injected along a major radius in the horizontal midplane, the ions generated by collisions will gyrate about a field line with almost no parallel velocity. The charge-exchange neutrals will fly off of the field lines in a direction tric. so related to the local pitch angle of the magnetic field. If a matrix of collimated chargeexchange neutral detectors are placed above or below the beam, the magnetic field line pitch profile can be determined. Jobes [28] proposed the use of a fast ion camera for imaging the fast neutrals emitted from the plasma; however, there is some question as to what one would use for "film" in the camera. Again, if $B_{\phi}$ is known, $B_{\theta}$ can be inferred. For a plasma with large shear of the magnetic field, it becomes difficult to adequately image the charge-exchange emission with standard machine access.

\section{High Frequency $E M$ Wave Probes}

Information about the magnetic field in the plasma can also be obtained with high frequency electromagnetic ( $E M)$ wave probing. Some of the techniques that have been proposed include optical mixing, optical tagging, laser scattering, laser fluorescence, and $E M$ wave polarimetry.

Optical Mixing Optical mixing is an extension of the low frequency wave probing discussed earlier, except that two high frequency waves are used. This technique

\footnotetext{
${ }^{3}$ This beam composition is due to the molecular origin of the particles and the various disassociation mechanisms that are possible. It was not as complex a beam as it might appear.
} 
has been proposed by Forman and Riesenfeld [29]. This is potentially useful for plasmas where resonances are inaccessible with low frequency waves. The method involves using two lasers whose frequencies exceed characteristic plasma frequency, but whose difference frequency matches some plasma resonance. If, for example, the upp : hybrid resonance is chosen, the appropriate laser combination can be used to excite upper hybrid oscillation at a location defined by the intersection of the lasers. The laser polarization effects are sensitive to the local magnetic field pitch angle in this case. There are no known experiments that have been performed to develop this technique; however, the electron plasma frequency has been excited by similar methods [30]. There is much more work required before the viability of this technique for magnetic field measurements can be established. The complete theory has not yet been done; the laser availability, in terms of wavelengths, coherence, and power, is still a question; and the plasma access required has not yet been determined.

Optical Tagging Stern, et al., $\{31]$ have proposed and developed a diagnostic technique where an ion can be "tagged" and the trajectory of that ion, or the magnetic field line on which it travels, can be determined. The method involves the use of a tunable dye laser to pump an ion into a metastable state at some location in the plasma. A second tunable laser is used to pump the ion from the metastable state to one which decays quickly. By scanning the second laser in space and time, the trajectory of the ion can be determined. This technique has been demonstrated on a $Q$-machine. For fusion plasmas there are problems finding an appropriate species, and problems with collisional excitation and deexcitation by the plasma. Also, a single-shot profile determination would require the use of several different species and laser combinations, and even finding one such combination is a challenge.

Laser Scattering There are two ways in which laser scattering can be used to obtain information about the internal magnetic field. The first is to make standard Thomson scattering measurements, aligning the differential scattering vector, $\vec{k}$, parallel to the local current. (For applications where the internal fields are not approximately known, this alignment is not possible.) The scattered spectrum is then shifted in wavelength due to the local electron drift velocity, from which the 
current density can be inferred. Although measurements have been made with this technique [32], there are very serious problems when the current carrying electrons are in the form of an enhanced tail of the distribution, as is the case with many current drive schemes, or in plasmas where there is a significant population of runaway electrons. If there is some assurance that the current is being carried by the thermal electrons, this technique may provide information about the plasma current density profiles and hence the internal magnetic field.

A second application of laser scattering for magnetic field measurements exploits the cyclotron modulation of the scattered light spectrum. When the differential scattering vector is nearly perpendicular to the field, and the wavelength is much less than the Larmor radius, then the spectrum is modulated by the cyclotron frequency due to the sinusoidal Doppler shift of the $E M$ wave scattered off the electrons executing Larmor orbits. The modulated spectrum may have very fine structure, imposing a requirement for an impractical number of wavelength channels to resolve the spectrum. Sheffield [33] has proposed the use of a Fabry-Perot etalon to transmit the component of the spectrum at the cyclotron frequency, eliminating the need to resolve the scattered spectrum in detail. Measurements of the magnetic field by this technique on the DITE tokamals are reported by Forrest, et al. [34].

One of the problems with this technique is the requirement for exceptional access to the plasma, unless the field lines are almost completely toroidal, as in a tokamak. Detailed knowledge of the magnetic field direction is required, because components of the field parallel to the differential wave vector allow electron thermal effects to smear the fine structure of the scattered spectrum. For plasmas with high shear, this is especially difficult. And, a priori knowledge of the field strength is required for setting the transmission frequency of the Falry-Perot etalon to the cyclotron frequency. All of these problems are accentuated if a multi-point system is considered for single-shot profile determination on a machine other than a tokamak.

Neutral Beam Laser Fluorescence Spectroscopy (Zeeman Splitting of Atomic Levels) This diagnostic technique uses both particle beam and EM wave probing. The principle on which this measurement is based was originally proposed as a passive diagnostic of the magnetic field. A particle in a magnetic 
field experiences Zeeman splitting of atomic levels. The wavelength shift from the levels in the absence of magnetic field is given by:

$$
\Delta \lambda \sim 5 \times 10^{-9} \lambda^{2} B
$$

with $B$ in Tesla and $\lambda$ in Angstroms. Therefore, by measuring the spectrum of line emission from a particle in a magnetic field, information about the field can be obtained.

A passive measurement that uses the Zeeman splitting of atomic levels was demonstrated by Wroblewski and Moos [35]. Line emission from magnetic dipole transitions in heavy plasma impurities was measured. These are long wavelength (visible) transitions, exploiting the $\lambda^{2}$ scaling of the Zeeman splitting. Inaccuracy in determining the spacial location of the impurities produces uncertainties in the measurements, and single-shot magnetic field profile measurements are not possible with this technique. Wroblewski and Moos [35] present measurements of the magnetic field on the TEXT tokamak to within $20 \%$ (the field is known to much better accuracy from the vacuum field relation).

Another particle that could be used for this measurement is lithium. This choice is motivated by the relatively simple emission spectrum of the $2 p-2 s$ transition of lithium, which is shown in Figure 1.2 as a function of applied magnetic field. The $\pi$ components are polarized parallel to the local magnetic field, therefore measurement of the polarization orientation of the $\pi$ emission indicates the magnetic field direction. The central $\pi$ components are particularly useful for this measurement because the wavelength of the emission is nearly independent of the magnetic field strength. This polarization measurement ( 7 .eeman polarimetry) is useful for a tokamak diagnostic where the field line pitch gives the pcloidal field strength; however, a more complete measurement of the emission spectrum (Zeeman spectroscopy) is also required for the determination of the magnetic field strength in more general plasmas.

In practice, it is necessary to impose active methods in order to generate enough emission to accurately resolve the spectrum, and to allow a determination of the spatial location of the emitting particles. A neutral lithium beam is injected into the plasma to provide a unique, spatially localized source. The measurement location is determined by the intersection of the beam and the detector line of sight. Although collisions could serve as the excitation mechanism, it is useful to optically excite the 


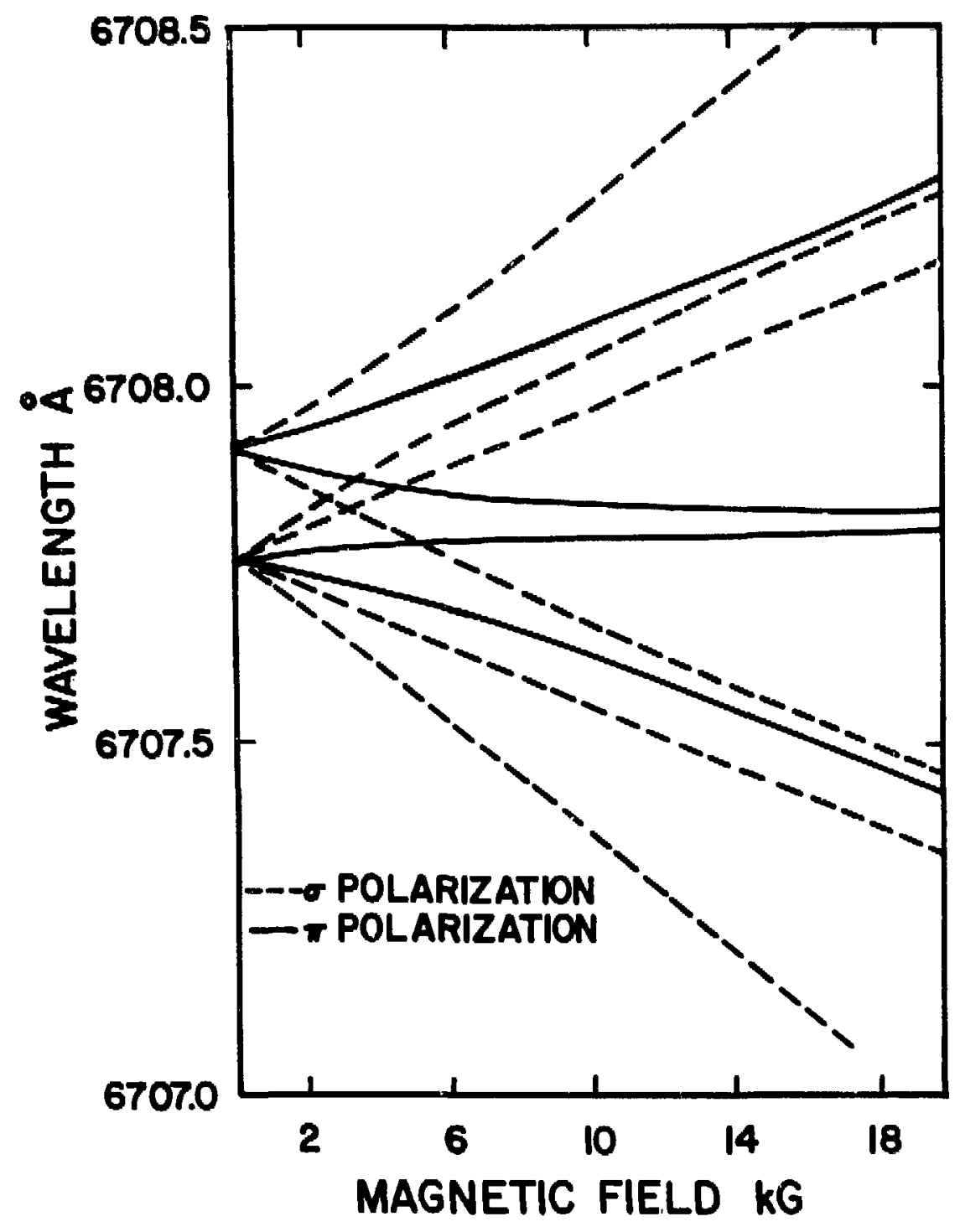

Figure 1.2: Zeeman Splitting of the Lithium $2 p-2 s$ Transition 
neutral lithium atoms with a tunable dye laser, to enhance the signal, and by laser modulation, also enhance the signal-to-noise. McCormick[36] has used collisional excitation for the system on ASDEX for making current density measurements, and although it is limited in comparison to dye laser enhanced systems, it is making routine measurements while the dye laser systems are being developed.

The primary issues in developing a magnetic field diagnostic that measures Zeeman splitting by neutral beam laser fluorescence spectroscopy are: 1) obtaining beam penetration to the central regions of the plasma, and 2) obtaining the cold, low divergence beam required to resolve the Zeeman spectrum. West, et al., [37] have calculated the beam requirements for Zeeman polarimetry to be a velocity spread $\Delta V \leq 100$ Volts and a beam divergence $\Delta \theta \leq 50 \mathrm{mrad}$. For a more complete measurement, Zeeman spectroscopy, the beam requirements were found to be more stringent, with $\Delta V \leq 1$ Volt and $\Delta \theta \leq 10 \mathrm{mrad}$. Even if this beam quality is achieved, Stark broadening of the spectral lines and stray light may smear or swamp the Zeeman spectrum, and make the determination of the magnetic field impossible. Also, like all other neuiral beam diagnostics, beam penetration problems in reactor grade plasmas may limit the ultimate use of this technique.

A Zeeman polarimetry experiment has been developed by West, et al., for a determination of magnetic field direction on TEXT, and some preliminary results have been published [38]. Due to neutral beam current limitations and beam attenuation in the plasma, only edge measurements were possible. The measurements closer to the center had poor signal-to-noise.

A more ambitious system is under development at Los Alamos [39] for magnetic field measurements on ZT-40M by Zeeman spectroscopy. Larger beam currents have been attained, which will allow measurements to be made in the central regions of the plasma. In addition, the system specifications are such that a measurement of the magnetic field strength should be possible, which is a requirement for magnetic field determination on a reversed field pinch.

Polarimetry The polarization of an $E M$ wave probe is altered upon propagation through the plasma in a way that depends on the magnetic field. Propagation along the field lines causes a rotation of the plane of polarization due to the Faraday effect, and propagation perpendicular to the field lines causes an elliptization of the polarization due to the Cotton-Mouton effect. The rotation induced by the 
plasma is given by:

$$
F=2.63 \times 10^{-25} \lambda^{2} \int_{L} n_{e} \vec{B} \cdot \overrightarrow{a l},
$$

and the elliptization is defined in terms of the angle of phase difference between the fast and slow components of the wave, which can be expressed as:

$$
\beta=2.45 \times 10^{-29} \lambda^{3} \int_{L} n_{c}|\vec{B} \times \widehat{d l}|^{2} d l,
$$

where the quantities $F$ and $\beta$ are in radians, and the other parameters are in MKS units, except for $\lambda$, which is in microns - reflecting the wavelength range that is commonly employed for magnetic field measurements based on polarimetry of $E M$ wave probes. Since the Faraday rotation and Cotton-Mouton effect also depend on plasma density, a simultaneous measurement of the density is required to extract the magnetic field information from the polarimetric measurements.

Conceivably, if the general changes in polarization of the $E M$ wave probe can be monitored, information about the chord-averaged parallel and transverse magnetic field can be obtained. Such a general polarization analyzer has not yet been developed, however the polarimetric techniques employed by Soltwisch [40] might be able to be extended to do this. Segre [41] has proposed a technique where the system has two distinct states - one state able to measure the rotation and the other able to measure the elliptization. If the state of the polarimeter is able to be changed back and forth on a timescale much faster than the plasma lifetime, both the parallel and perpendicular magnetic fields can be measured.

Efforts in developing a capability for polarinetric measurements of the magnetic field have focused primarily on the measurement of Faraday rotation. The main reason for this is that detectable Faraday rotation can be generated by the plasma on a wave probe of short enough wavelength that severe refractive bending can be avoided. Longer wavelengths are required to produce measurable ellipticity, where refractive bending can make the measurement impossible unless ample diagnostic access is available. Also, most of the polarimetry development has been done on tokamak plasmas, where a measurement of the toroidal field is not of interest, and standard diagnostic access is appropriate for Faraday rotation measurements of the poloidal magnetic field. In a reversed field pinch, measurement of the toroidal field would also be useful; however, due to the refractive effects mentioned above, techniques other than polarimetry need to be considered for the measurement of the toroidal field, unless toroidal access to the plasma is available. 
The simplest way to measure the Faraday rotation is to place a linear polarization analyzer in the path of a linearly polarized $E M$ wave probe after it has propagated through the plasma. If the analyzer is adjusted to achieve a null transmission in the absence of plasma, the Faraday rotation will generate a polarization component that will be transmitted by the analyzer. The $E M$ wave intensity measured by a detector beyond the analyzer is then related to the Faraday rotation by the following equation:

$$
I=I_{o} \sin ^{2} F,
$$

where $I_{o}$ is the original intensity of the $E M i$ wave, and $F$ is defined in Eq. 1.6. In practice, it is found useful to modulate the intensity sensed by the detector at some known frequency to facilitate detection and enhance signal-to-noise ratios. This is accomplished either by zodulating $I_{o}$ in time [40], or by rocking the polarization angle of the inciderit $E M$ wave, which generates a sinusoidal temporal modulation of the intensity transmitted by the polarization analyzer $[42],[43]$. Both of these concepts will be discussed in detail in Section 3.2.1.

A fundamental problem with these amplitude methods is that there are other effects that may alter the intensity of the $E M$ wave probe before it reaches the analyzer. This directly induces errors in a Faraday rotation measurement. Although absorption by the plasma is usually a small effect for conditions of interest, slight refractive bending can result in lost intensity on the detector. This fact was recognized early in the development of interferometry for plasma density measurements, and the implementation of heterodyne phase shift methods nearly eliminated the sensitivity to spurious changes in the $E M$ wave probe intensity. In heterodyne phase shift interferometry, the plasma density changes alter the time signature of the modulated intensity sensed by the detector, and the measurement is not affected by spurious changes in the signal amplitude. Heterodyne techniques can also be applied to polarimetry, and Faraday rotation measurements can be made even though there are effects that may alter the intensity of the $E M$ wave probe before it reaches the detector. The Faraday rotation appears as a phase shift of the modulated intensity sensed by the detector.

For any of the Faraday rotation measurement methods described above, there are optical configurations that allow a simultaneous measurement of the plasma density. (Even if amplitude polarimetry methods are used, the interferometry 
measurements are made with heterodyne phase-shift methods.) This allows $B_{\theta}$ to be measured with standard diagnostic access.

There have been several experimental programs with a goal of developing and implementing a Faraday rotation diagnostic for poloidal magnetic field measurement. The most impressive system to date is the polarimeter/interferometer on the TEXTOR tokamak developed by Soltwisch [40]. The system is based on a heterodyne amplitude polarimetry method (see Figure 3.4) rather than the more sophisticated heterodyne phase-shift techniques, but the reliable multichord system is actually used as the plasma position monitor for the feedback control of the plasma position [44]. A similar system is being implemented on the TFTR tokamak, where the extension of the FIR system to simultaneous polarimetry/interferometry is being pursued now that the primary task of assembling the interferometer has been completed [45].

The rocking polarization method (see Figure 3.5) was first demonstrated by Kunz [42]. Extensive systems based on this technique (and incorporating simultaneous interferometric measurements) have been assembled on the ISX-B tokamak [46][43] and on TEXT tokamak [47]. Initial measurements from these multichord polarimeter/interferometer indicated that the current density profiles were consistent with Spitzer resistivity, but they have not made routine measurements beyor.d that point.

Optical configurations that allow both polarimetry and interferometry measurements by heterodyne phase-shift methods have been proposed [48][49]. Heterodyne phase-shift polarimetry was first demonstrated by Fukuda, et ul.[50] on a fieldreversed theta pinch plasma. Although the initial measurements were successful from a diagnostic development standpoint, the Faraday rotation was dominated by unconfined plasma streaming out the ends of the device. Therefore, the measurements did not contribute to the understanding of confinement physics, and further measurements were not pursued.

A multichord heterodyne phase-shift polarimeter/interferometer was developed by Young, et al., at UCLA [51]. Although the density profile measurements were useful, some problems were found with the Faraday rotation measurements. The apparent rotation on adjacent channels did not correlate as one would predict. These problems were observed at levels well below the resolution of the Fukuda instrument. The cause of the problem was not well understood, though some con- 
jectures of possible causes were made. Gomez and Wolfe [52] proposed a heterodyne technique that was tested on ALCATOR C. Work was not continued beyond the initial test because of sensitivity problems that were observed.

This thesis describes work performed to develop such a measurement system on ZT-40M. As will be discussed, initial problems in polarimetric sensitivity were encountered, also below the resolution of the Fukuda instrument. Once the sensitivity problems were solved, useful Faraday rotation measurements were possible. The demonstration of simultaneous polarimetry and interferometry by heterodyne phase-shift methods was also successful. More details about the theory behind the measurement, and a desciption of the experimental development that was required to make the successful measurement will be presented in the remainder of this thesis.

\subsubsection{Relative Merits of Measurement Techniques}

Ideally, a diagnostic must provide a local measurement of the magnetic field, and provide accurate, single-shot profile information without a great deal of complexity. It should be able to make measurements on any plasma configuration because there is no real certainty as to which of the present confinement schemes might develop into an economically competitive reactor. (The next generation machines may not be tokamaks.) Also, the developmental status of the measurement technique needs to be assessed. Indeed, some techniques have not been demonstrated, and several issues need to be resolved before success can be predicted. It is important that the measurements be made with standard diagnostic access (or at least access that can be obtained without perturbation to the mechanical structure or plasma confinement). Consideration also needs to be given to the potential of each technique to make measurements of reactor-grade plasmas. This is not as serious a constraint as some of the others, due to the interest in magnetic field measurements in laboratory plasmas.

The techniques that have been proposed for the measurement of the magnetic field are listed in Table 1.1. Notations are made when a given technique has a problem meeting the constraints discussed above. Distinctions are made when the technique has a fundamental problem meeting a constraint, which may preclude the generation of accurate and useful information. It doesn't take any effort to 


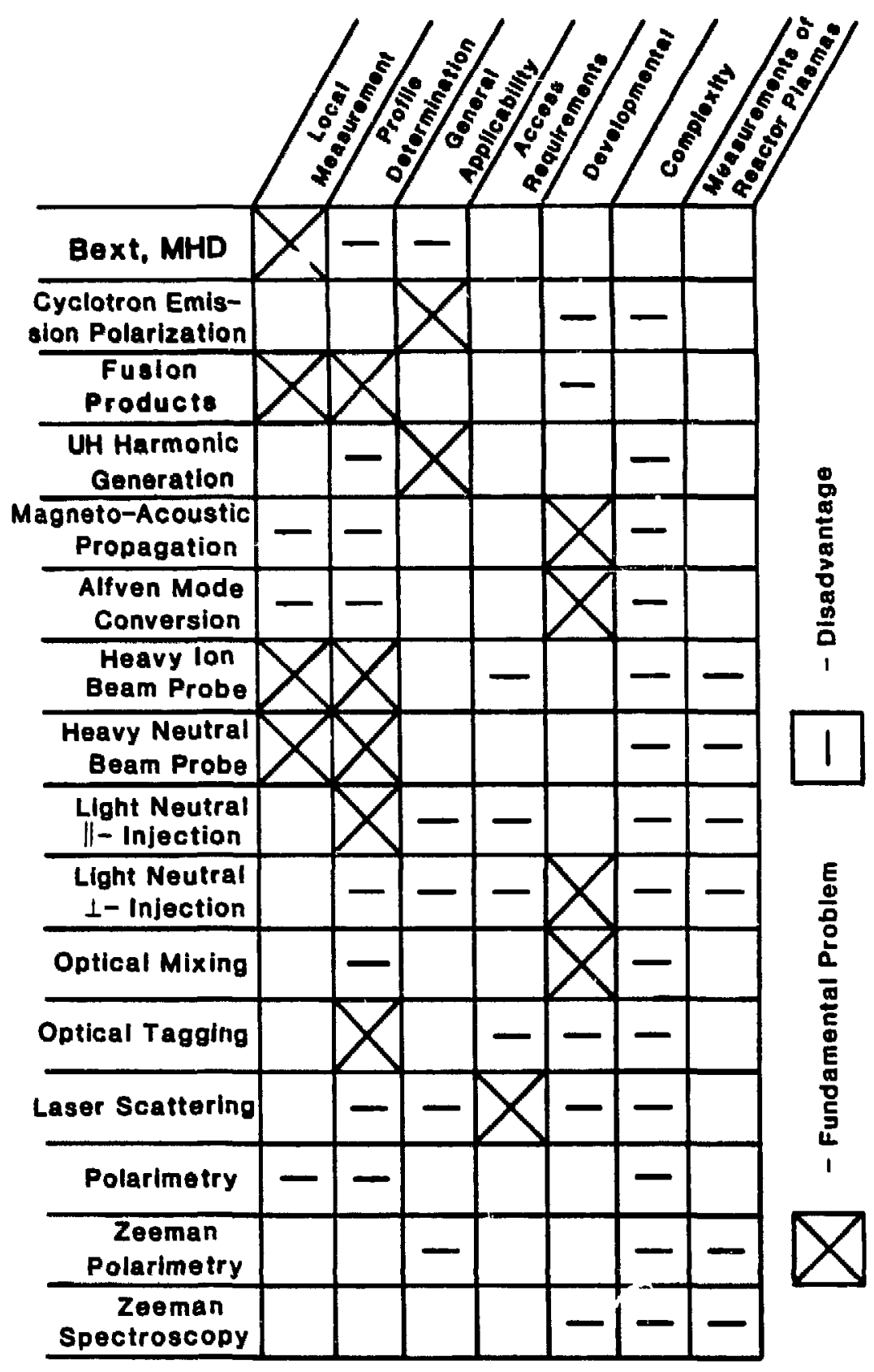

Table 1.1: Relative Merits of Measurement Techniques 
conclude that none of the options clearly stand out as being the optimal magnetic field diagnostic.

Perhaps the most promising options are Faraday rotation and Zeeman spectroscopy. Faraday rotation does have the disadvantage of requiring an inversion of the data to recover the magnetic field information, but it only requires diagnostic access that is standard on most machines (for a poloidal field measurement), and can be readily used in the next generation of plasmas. Zeeman spectroscopy is still developmental, but does have the potential of making unambiguous measurements at a well-defined point in space; therefore it is not prone to uncertainties in a complicated inversion process. Although it may have diffculties with beam penetration in fusion-reactor plasmas, it will probably be able to provide useful magnetic field measurements in the present generation of plasma machines. The measurement of the field direction by Zeeman polarimetry may be possible even if problems in Zeeman spectroscopy are encountered. In this case, Faraday rotation and Zeeman polarimetry are useful complementary diagnostics, with Faraday rotation measurements for poloidal field determination and Zeeman polarimetry for field direction, and the two together allowing a determination of the total internal magnetic field in a general plasma.

The following chapters will deal more fully with the theory behind Faraday rotation measurements, techniques for measuring the Faraday rotation and plasma density simultaneously, and potential sources of errors. Techniques for inverting Faraday rotation measurements will be discussed, and error propagation in the inversion will be studied. This defines the required system performance for adequate profile determination. The system used for making Faraday rotation measurements on the reversed-field pinch ZT-40M will be described, along with an account of the development that was required to obtain sufficient sensitivity with the system. Simultaneous Faraday rotation and density measurements will be presented, and recommendations for future work will be outlined. 


\section{Chapter 2}

\section{Theory}

Interferometry and polarimetry are techniques that measure relative changes in the phase and polarization of an electromagnetic $(E M)$ wave. To determine how these techniques can be used to measure plasma parameters, the effects that a plasma has on the phase and polarization of $E M$ waves need to be investigated. These effects are expressed by Appleton's equation, which is a dispersion relation for $E M$ waves in a magnetized plasma. The dominant effect of the plasma depends only on the plasma density, which alters the phase velocity of the $E M$ wave. This effect can be readily measured. Retention of higher order terms in Appleton's equation reveals that propagation along the magnetic field results in a rotation of polarization azimuth (Faraday effect), and propagation perpendicular to the magnetic field causes changes in the polarization ellipticity ${ }^{1}$ (Cotton-Mouton effect). Therefore, analysis of the polarization changes induced by the plasma (polarimetry) will give information on the magnetic field within the plasma.

For most fusion plasmas, the Cotton-Mouton effect is difficult to measure, because the effect is very small for radiation wavelengths that don't suffer severe refractive bending. Faraday rotation measurements are more likely to be successful, because measurable rotation is induced at wavelengths that are less sensitive to refractive bending. Techniques for measuring the plasma density a:d Faraday effect via $E M$ wave probing will be discussed in Chapter 3, alnng with sources of errors that may affect the accuracy of these measurements.

Measurements made by $E M$ wave probing are chord-averaged. ${ }^{2}$ Therefore, measurements need to be made on several chords, and these measurements need to

\footnotetext{
${ }^{1}$ This polarization terminology is defined in Appendix $\mathbf{B}$.

${ }^{2} \mathrm{~A}$ chord is a linear trajectory through the plasma.
} 
be inverted in order to obtain spatial profiles of the plasma parameters. Techniques for inverting chord-averaged data will be describeà below.

The measurement accuracy required for adequate profile determination depends on how errors propagate in the inversion process. Methods for studying error propagation will be described, and conclusions based on relatively simple techniques will be presented.

\subsection{Propagation of Electromagnetic Waves in a Magnetized Plasma: Appleton's Equation}

The derivation of Appleton's equation appears in many textbooks (e.g.,[53],[54]). The important aspects of the derivation will be presented here, primarily to state the assumptions that are commonly used, thereby establishing the region of validity of the theory.

There are two different approaches that can be taken to derive Appleton's equatior. The approach that perhaps offers more insight involves a derivation of the wave equation from Maxwell's equation, and a derivation of the dielectric response of the plasma from a set of equations that describe the plasma.

The other approach uses the same equation to derive the plasma response. However, rather than expressing the response in a dielectric tensor, it is represented by a conductivity tensor in a form of Ohm's law. A'so, rather than deriving the wave equation, Maxwell's equations are used to form another expression of Ohm's law. The statemait of equivalence of the two forms of Ohm's law then results in Appleton's equation. This second approach is entirely equivalent to the wave equation method; however, it involves less algebra, and is the method that will be used below. 


\subsubsection{Ohm's Law from Maxwell's Equations}

For problems in electromagnetic theory, it is conventional to write Maxwell's equations for the medium as follows:

$$
\begin{aligned}
\nabla \cdot \vec{D} & =\rho \\
\nabla \times \vec{E} & =-\frac{\partial \vec{B}}{\partial t} \\
\nabla \cdot \vec{B} & =0 \\
\nabla \times \vec{H} & =\vec{J}+\frac{\partial \vec{D}}{\partial t} \\
\vec{D} & =\overline{\bar{\epsilon}} \cdot \vec{E} \\
\vec{B} & =\overline{\bar{\mu}} \cdot \vec{H} \\
\vec{J} & =\overline{\bar{\sigma}} \cdot \vec{E},
\end{aligned}
$$

where $\rho$ and $\vec{D}$ represent unbound charge and current densities. The bound charge and current densities resulting from polarization and magnetization are embodied in the vector quantities $\vec{D}$ and $\vec{H}$, in terms of the constitutive parameters $\overline{\bar{\epsilon}}$ and $\overline{\bar{\mu}}$. In most media, the constitutive parameters can be empirically determined, and no detailed information is required concerning the effects on the microscopic scale of individual particles.

In a plasma, the constitutive parameters are not easily measured, nor are they simple constants, as is the case with most media that consist of neutral particles. Hence, it is more convenient to deal with a plasma with free and bound charge and eurrent densities included in the terms $\rho$ and $\vec{J}$. Detailed knowledge of the behavior of individual particles is then required in order to calculate the charge and current density through

$$
\rho=\sum_{i} n_{i} q_{i} \quad \text { and } \quad \vec{J}=\sum_{i} n_{i} q_{i} \vec{v}_{i},
$$

where $i$ denotes the species subscript, $n$ is the density, $q$ the charge, and $\vec{v}$ the velocity. In this case, the constitutive parameters take on the values they have in a vacuum, $\epsilon_{o}$ and $\mu_{o}$. Maxwell's equations can then be written:

$$
\begin{aligned}
\nabla \cdot \vec{E} & =\rho / \epsilon_{o} \\
\nabla \times \vec{E} & =-\frac{\partial \vec{B}}{\partial t} \\
\nabla \cdot \vec{B} & =0 \\
\nabla \times \vec{B} & =\mu_{o} \vec{J}+\mu_{o} \epsilon_{o} \frac{\partial \vec{E}}{\partial t} .
\end{aligned}
$$

Note that Maxwell's equations for a plasma are equivalent to the equations that would be written if the plasma were considered to be a vacuum where charges and currents could exist. 
Maxwell's equations will be used to describe the effects of waves in a plasma. Although there are usually equilibrium or static values of the fields and currents, Eq. 2.3 applies to the static values and wave values separifely due to superposition properties of Maxwell's equations, and the field quantities of Eq. 2.3 will be taken to represent the wave from now on.

Maxwell's equations can be converted from differential expressions to algebraic expressions if certain approximations are made. The plasma is assumed to be homogeneous and of infinite extent. It is further assumed that the perturbations associated with the wave are small. This allows the equations to be linearized, so that a sum of solutions is also a solution. This allows treatment of each wave separately, and solutions to Eq. 2.3 will be in the form of plane monochromatic waves, where the quantities can be written as

$$
\vec{E}(\bar{x}, t)=\vec{E}(\vec{k}, \omega) e^{i(\vec{k} \cdot \vec{x}-\omega t)}
$$

If it is assumed that $\vec{k}=k \hat{z}$, where $\hat{z}$ is the unit vector in the $z$-direction, then Faraday's law and Ampere's law in component form become

$$
\begin{aligned}
-i k E_{y} & =i \omega B_{x} \\
i k E_{x} & =i \omega B_{y} \\
0 & =i \omega B_{z} \\
-i k B_{y} & =\mu_{o} J_{x}-i \omega \mu_{o} \epsilon_{o} E_{x} \\
i k B_{x} & =\mu_{o} J_{z}-i \omega \mu_{o} \epsilon_{o} E_{y} \\
0 & =\mu_{o} J_{z}-i \omega \mu_{o} \epsilon_{o} E_{z} .
\end{aligned}
$$

Eliminating $B_{x}$ and $B_{y}$, with $c=1 / \sqrt{\mu_{o} \epsilon_{o}}$ being the speed of light in a vacuum and defining $N=k c / \omega$, Equation 2.5 becomes

$$
-i \omega \epsilon_{o}\left(\begin{array}{ccc}
N^{2}-1 & 0 & 0 \\
0 & N^{2}-1 & 0 \\
0 & 0 & N^{2}-1
\end{array}\right)\left(\begin{array}{c}
E_{x} \\
E_{y} \\
E_{z}
\end{array}\right)=\left(\begin{array}{c}
J_{x} \\
J_{y} \\
J_{z}
\end{array}\right) .
$$

This is seen to be a form of Ohm's law and can be written as

$$
\overline{\bar{\sigma}} \cdot \vec{E}=\vec{J} .
$$

It is now necessary to determine the relationship between the current and the electric field in the plasma. 


\subsubsection{Plasma Resistivity from Fluid Theory}

The set of equations that describe a plasma can contain a wide spectrum of complexity, from simple fluid equations with ion motion and collisions neglected to a formal kinetic description of the plasma through Boltzman's equation. The level of complexity required is governed by the effects that are being investigated. The simple set of fluid equations is adequate to describe the effects of the plasma on $E M$ wave propagation for the wave frequencies of interest. The ion response to the wave fields can be ignored due to their large inertia compared to electrons. Collisional effects can also be ignored when the collision frequency is much less than the $E M$ wave frequency. This condition is certainly valid for most fusion plasmas and relevant wave frequencies, and therefore collisional effects will be ignored for the derivation of Appleton's equation. The neglect of collisions is equivalent to setting the plasma temperature to zero in the fluid equations, and therefore this is commonly referred to as the cold plasma approximation. The neglect of temperature effects can be justified directly because the $E M$ wave phase velocity is much greater than the thermal velocities of the particles.

The important equation from fluid theory is the equation of motion for the electrons, which is given by

$$
\frac{\partial \vec{v}}{\partial t}=\frac{q}{m}(\vec{E}+\vec{v} \times \vec{B})
$$

where $q$ is the electronic charge, $m$ and $\vec{v}$ are the mass and velocity of the electron, and $\vec{E}$ and $\vec{B}$ represent the total fields in the plasma. The equation of motion can be linearized through perturbation analysis if it is assumed that the velocity and field quantities can be expressed as a summation of terms of successively higher order (i.e., $\vec{v}=\vec{v}_{0}+\vec{v}_{1}+\vec{v}_{2}+\cdots$ ). Here, the zero order represents the static quantity, and the first order represents the much smaller quartities associated with the wave. Equation 2.8 can then be separated into an equilibrium part (zeroth order terms) and a perturbed part (first order terms and terms containing products of zeroth and first order quantities). It is assumed that the plasma is at rest and there is no static electric field $\left(\vec{v}_{\circ}=0, \vec{E}_{\circ}=0\right)$. The equilibrium equation is then trivial, and the perturbation equation is:

$$
\frac{\partial \vec{v}_{1}}{\partial t}=-\frac{e}{m}\left(\vec{E}_{1}+\vec{v}_{1} \times \vec{B}_{o}\right) .
$$




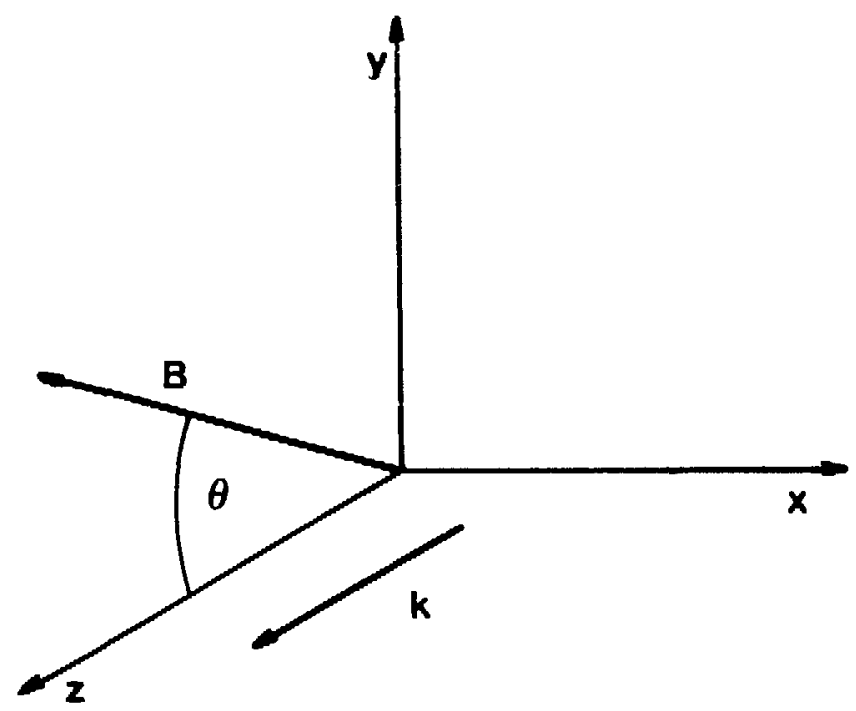

Figure 2.1: Coordinate System

If we orient = cartesian coordinate system such that $B_{0}$ lies in the $y-z$ plane, with $\vec{B}=B_{o}(U, \sin \theta, \cos \theta)$ (see Fig. 2.1), and assume plane monochromatic waves, Eq. 2.9 can be written in component form as follows

$$
\begin{aligned}
-i \omega v_{x}+\frac{e B_{0}}{m} \cos \theta v_{y}-\frac{e B_{o}}{m} \sin \theta v_{z} & =-\frac{e}{m} E_{x} \\
-\frac{e B_{0}}{m} \cos \theta v_{x}-i \omega v_{y} & =-\frac{e}{m} E_{y} \\
\frac{e B_{0}}{m} \sin \theta v_{x} &
\end{aligned}
$$

where the subscripts designating perturbed quantities have been dropped. Replacing the velocity with current density $\vec{J}=-n e \vec{v}$ and defining $\Omega=e B / m$, the electron cyclotron frequency, the equation of motion becomes:

$$
\frac{m}{n e^{2}}\left(\begin{array}{ccc}
-i \omega & \Omega \cos \theta & -\Omega \sin \theta \\
-\Omega \cos \theta & -i \omega & 0 \\
\Omega \sin \theta & 0 & -i \omega
\end{array}\right)\left(\begin{array}{c}
J_{x} \\
J_{y} \\
J_{z}
\end{array}\right)=\left(\begin{array}{c}
E_{x} \\
E_{y} \\
E_{z}
\end{array}\right)
$$

Equation 2.11 is another form of Ohm's law, where the tensor represents the plasma 
resistivity; therefore, Eq. 2.11 can be written in the abbreviated form:

$$
\overline{\bar{\eta}} \cdot \vec{J}=\vec{E} .
$$

The form of Ohm's law derived in Section 2.1.1 now needs to be coupled with this result. The statement of equivalence between Eq. 2.7 and Eq. 2.12 leads to an expression known as Appleton's equation.

\subsubsection{Appleton's Equation}

Maxwell's equations relate the fields to the charges and the currents in a plasma, and the equation of fluid theory relate the fields to the dynamical motion of the particles. This set of equations must be solved simultaneously for the self-consistent behavior of the particles. Only then can the constitutive parameters be derived.

Substituting Eq. 2.7 into Eq. 2.12, the following is obtained:

$$
\vec{E}=\overline{\bar{\eta}} \cdot \vec{J}=\overline{\bar{\eta}} \cdot \overline{\bar{\sigma}} \cdot \vec{E},
$$

or

$$
(\overline{\bar{\eta}} \cdot \overline{\bar{\sigma}}-\overline{\bar{I}}) \cdot \vec{E}=0,
$$

where $\overline{\bar{I}}$ represents the unit tensor. Equation 2.14 represents three simultaneous hornogeneous equations; therefore, the non-trivial solution requires that the determinant of the coefficient matrix be zero. Performing the matrix multiplication, and defining $\omega_{\mathrm{p}}^{2}=n e^{2} / \epsilon_{\mathrm{o}} m$, the determinantal equation becomes:

$$
\frac{-i \omega}{\omega_{p}^{2}}\left|\begin{array}{ccc}
-i \omega\left(N^{2}-1\right)-i \omega_{p}^{2} / \omega & \Omega\left(N^{2}-1\right) \cos \theta & \Omega \sin \theta \\
-\Omega\left(N^{2}-1\right) \cos \theta & -i \omega\left(N^{2}-1\right)-i \omega_{p}^{2} / \omega & 0 \\
\Omega\left(N^{2}-1\right) \sin \theta & 0 & i \omega-i \omega_{p}^{2} / \omega
\end{array}\right|=0 .
$$

Dimensionless parameters are conventionally defined in the following way to simplify the notation:

$$
M^{2}=N^{2}-1, Y=\frac{\Omega}{\omega}, Y_{L}=Y \cos \theta, Y_{T}=Y \sin \theta, X=\frac{\omega_{p}^{2}}{\omega^{2}} .
$$

Equation 2.15 then becomes:

$$
-\frac{M^{2}}{X}\left|\begin{array}{ccc}
1+X / M^{2} & i Y_{L} & i \frac{Y_{I}}{M^{2}} \\
-i Y_{L} & 1+X / M^{2} & 0 \\
i Y_{T} & 0 & -\frac{(1-X)}{M^{2}}
\end{array}\right|=0
$$


Evaluation of the determinant results in the following expression:

$$
(1+X / M)^{2}-Y_{L}^{2}-\frac{Y_{T}^{2}}{1-X}\left(1+X / M^{2}\right)=0 .
$$

Solving for $\left(1+X / M^{2}\right)$ yields:

$$
1+X / M^{2}=1 / 2-\frac{Y_{T}^{2}}{1-X} \pm\left[\frac{Y_{T}^{4}}{4(1-X)^{2}}+Y_{L}^{2}\right]^{1 / 2}
$$

and,

$$
M^{2}=-\frac{X}{\left[1-\frac{Y_{T}^{2}}{2(1-X)}\right] \pm\left[\frac{Y_{T}^{4}}{4(1-X)^{2}}+Y_{L}^{2}\right]^{1 / 2}}
$$

Returning to the original notation and solving for $N^{2}$ gives

$$
N^{2}=1-\frac{X}{\left[1-\frac{\left(\Omega^{2} / \omega^{2}\right) \sin ^{2} \theta}{2\left(1-\omega_{p}^{2} / \omega^{2}\right)}\right] \pm\left[\frac{\left(\Omega^{4} / \omega^{4}\right) \sin ^{4} \theta}{4\left(1-\omega_{p}^{2} / \omega^{2}\right)^{2}}+\frac{\Omega^{2}}{\omega^{2}} \operatorname{css}^{2} \theta\right]^{1 / 2}} .
$$

This result is known as Appleton's equation, which represents the dispersion relation for a cold, magnetized plasma. This equation contains the information about the effects the plasma has on $E M$ wave propagation. The equation depends only on the electron density and magnetic field (all other parameters have been shown to have negligible effects for $E M$ wave frequencies and plasmas of interest). Next, limiting cases of this equation will be investigated to give insight on how these plasma parameters can be measured by $E M$ wave probing.

\subsection{Limiting Cases of Appleton's Equation ${ }^{3}$}

\subsubsection{Leading order depends only on density}

Examination of Appleton's equation indicates that the dominant contribution a plasma has on the propagation of an $E M$ wave depends only on density. It is useful to consider the approximate values of the dimensionless parameters used to derive Appleton's equation. The two important parameters, $x$ and $y$, have the

\footnotetext{
${ }^{3}$ Throughout this section, terminology and mathematical operations dealing with polarisation effects will be used. The reader who is not already familiar with polarization formalism is referred to Appendix B, where the crystal-optic analogy of a plasma is described, and mathematical methods for polarization description and evolution are presented.
} 
following values for a plasma with a density of $10^{20} \mathrm{~m}^{-3}$ immersed in a 1 Tesla magnetic field, and for radiation at a wavelength of $185 \mu \mathrm{m}$ :

$$
X=\frac{\omega_{p}^{2}}{\omega^{2}} \simeq 1 / 325 \quad Y=\frac{\Omega}{\omega} \simeq 1 / 600 .
$$

The leading order contribution to the refractive index of the plasma is then:

$$
N^{2}=1-X=1-\frac{n_{c} e^{2}}{m \epsilon_{o} \omega^{2}} .
$$

In this limit the plasma is isotropic, and there are an infinity of characteristic wave polarizations that may propagate unaltered (see Section B.1). Therefore, no polarization changes of the $E M$ wave are generated in this case. The neglected terms are of order $Y^{2}$, or $\sim 3 \times 10^{-6}$. Were the higher order terms retained, they would result in very small corrections to the density contribution to the refractive index.

The density can therefore be determined very precisely, in most cases limited only by the accuracy to which the phase shifts imposed on the $E M$ wave probe can be characterized. This has led to the widespread and common application of $E M$ wave probing for density measurements on plasma experiments. Techniques for determining the density by refractive index measurements will be discussed later.

The total phase shift generated by a plasma of length $L$ is related to the density by:

$$
\phi=\frac{\omega}{c} N L=\frac{\omega L}{c}\left(1-\frac{n_{e} e^{2}}{\epsilon_{o} m \omega^{2}}\right)^{1 / 2} .
$$

This result was derived under the assumption that the plasma is infinite and homogeneous. In order to account for the finite extent of the plasme, the WKB approximation is comrnonly used. The WKB approximation is used extensively in quantum mechanics when solutions to the Schrödinger Wave Equation are of interest. A detailed description of the WKB method, along with the original references to the work of Wentzel, Kramers, and Brillion, appears in Schiff[55]. For the present purpose, the WKB approximation allows integration of the differential phase shift over the pathlength through the plasma, without consideration of the non-uniformities or gradients that are a result of the finite extent of the plasma.

In order for the WKB method to be valid, the typicai gradient scale length needs to be large compared to a wavelength. The conventional expression of this 
statement is:

$$
\frac{\lambda}{4 \pi} \nabla \vec{k} \ll|\vec{k}|
$$

or the fractional change in $\vec{k}$ over the distance of $\lambda / 4 \pi$ is much less than unity. If sharper gradients in the refractive index are encountered, partial reflection of the radiation results.

The WKB method allows the phase shift generated by the plasma to be written as:

$$
\phi=\int_{L_{1}}^{L_{2}} \vec{k} \cdot \overrightarrow{d l}
$$

or

$$
\phi=\frac{\omega}{c} \int_{L_{1}}^{L_{2}}\left(1-\frac{n_{e} e^{2}}{\epsilon_{o} m \omega^{2}}\right)^{1 / 2}|\vec{d}| .
$$

\subsubsection{Propagation along the magnetic field lines: Faraday Rotation}

Reexamination of the Appleton equation (Eq. 2.21) reveals that there are many more effects on the character of the $E M$ wave as it propagates through the plasma. These other effects are of higher order $\left(\sim Y^{2}\right)$, and thus produce smaller changes to the $E M$ wave probe. These changes, however, are caused by the local magnetic field, which is not well diagnosed on present experiments. Most methods for measuring the internal magnetic field are difficult to implement and/or difficult to interpret unambiguously (see Chapter 1). It is therefore of interest to investigate these higher order effects that the plasma has on an $E M$ wave, and how the effects might be exploited for making measurements of the internal magnetic field.

Setting $\theta=0$ (see Figure 2.1) in Appleton's equation (Equation 2.21) results in the following expression:

$$
N^{2}=1-\frac{\omega_{p}^{2} / \omega^{2}}{1 \pm \Omega / \omega}
$$

Recalling that $N^{2}=k^{2} c^{2} / \omega^{2}$, and solving for $k$, this becomes

$$
k=\frac{\omega}{c}\left[1-\frac{\omega_{p}^{2} / \omega^{2}}{1 \pm \Omega / \omega}\right]^{1 / 2}
$$


The characteristic polarization for this case can be found through Eq. 2.17 to be (see Appendix B.1):

$$
\frac{E_{x}}{E_{y}}=\frac{1+X / M^{2}}{i Y}=\frac{ \pm Y}{i Y}=\mp i
$$

The characteristic polarizations are seen to be right-handed and left-handed circular polarizations, and the phase velocity of these waves differ, as can be seen from Eq. 2.28. Hence in propagation along the magnetic field lines the plasma exhibits circular birefringence (see Appendix B.1). Therefore, a linearly polarized $E M$ wave will undergo a rotation of azimuth upon traversing a distance $\mathrm{z}$ in a uniform plasma, (known as Faraday rotation), with the angle of rotation given by (see Appendix B.2):

$$
F=\Delta / 2=\frac{k_{+}--k_{-}}{2} z,
$$

where $\Delta$ is the phase difference between the characteristic polarizations (as defined in Appendix B.2). Approximating the $k$ values through expansion of Equation 2.29 gives

$$
k \simeq \frac{\omega}{c}\left[1-\frac{1}{2} \frac{\omega_{p}^{2}}{\omega^{2}}\left(1 \mp \frac{\Omega}{\omega}+\cdots\right)\right],
$$

and the rotation becomes

$$
F=\frac{\Omega \omega_{p}^{2}}{2 c \omega^{2}} z=\frac{e^{3}}{2 \epsilon_{g} c m^{2} \omega^{2}} n_{e} B_{\|} z
$$

Therefore, by measuring the rotation of the polarization azimuth of the $E M$ wave upon transversal through the plasma parallel to the magnetic field, information about the $n_{e} B_{\|}$product can be obtained. With an independent measurement of the density, the magnetic field information can be extracted.

\subsubsection{Propagation transverse to magnetic field: Cotton- Mouton Effect}

Setting $\theta=90^{\circ}$ in Appleton's equation 2.21 yields the following expression:

$$
N^{2}=1-\frac{\omega_{p}^{2} / \omega^{2}}{\left[1-\frac{n^{2} / \omega^{2}}{2\left(1-\omega_{p}^{2} / \omega^{2}\right)}\right] \pm\left[\frac{n^{2} / \omega^{2}}{2\left(1-\omega_{p}^{2} / \omega^{2}\right)}\right]} .
$$

\footnotetext{
"Recall that the matrix of Equation 2.17 is the coefficient matrix for $\vec{E}$. The characteristic polarization is found most easily from the second of the three implied equations (Equation 2.30 also uses the first of the implied equations).
} 
Taking the negative sign, the refractive index again depends only on density:

$$
N^{2}=1-\omega_{p}^{2} / \omega^{2}
$$

This particular case is called the ordinary mode ( $O$-mode) since the expression is identical to the case when no magnetic field is present. Examination of Eq. 2.17 reveals that this solution comes from the second of the three implied equations, and that the characteristic wave is linearly polarized in the $y$-direction, or with the polarization parallel to $\vec{B}_{0}$.

Magnetic field effects enter into the second solution, namely:

$$
N^{2}=1-\frac{\omega_{p}^{2} / \omega^{2}}{1-\frac{n^{2} / \omega^{2}}{1-\omega_{p}^{2} / \omega^{2}}} .
$$

This wave is known as the extraordinary mode ( $X$-mode). The characteristic wave is seen to have a longitudinal component (see Appendix B.1 and Equation 2.17), with:

$$
\frac{E_{z}}{E_{x}}=-\frac{i X Y}{1-X-Y^{2}} .
$$

The fact that this ratio is imaginary indicates that the wave is elliptically polarized. The mathematical techniques described above are only able to determine changes in the polarization of transverse $E M$ waves, and therefore the presence of an electrostatic component in the characteristic polarization poses a problem. It is common practice to ignore the electrostatic component of this wave and treat it as though it was a transverse $E M$ wave. Referring back to Eq. 2.22 it is realized that the electrostatic component is indeed small for the plasma parameters and radiation wavelengths of interest. If this were not the case, the formalism for determining electrostatic polarization effects would need to be inciuded.

With the neglect of electrostatic effects, the characteristic polarizations are linear, with the $O$-mode polarized parallel to the transverse magnetic field, and the $X$-mode polarized perpendicular to the transverse magnetic field. In addition, since there is a difference in the refractive index for the $O$-mode and the $X$-mode, the plasma exhibits linear birefringence when the beam is propagated perpendicularly to the magnetic field.

The level of birefringence is defined in terms of the phase difference between the characteristic waves upon traversing a distance in the plasma (see Appendix B.2). 
For a distance $z$ in a uniform plasma, this phase shift is given by:

$$
\beta=\left(k_{o}-k_{x}\right) z .
$$

From Eq. 2.35, $k_{\text {。 }}$ is found to be:

$$
k_{o}=\frac{\omega}{c}\left(1-\omega_{p}^{2} / \omega^{2}\right)^{1 / 2} \simeq \frac{\omega}{c}\left(1-\omega_{p}^{2} / 2 \omega^{2}\right),
$$

and from Eq. 2.36, $k_{x}$ is:

$$
k_{x}=\frac{\omega}{c}\left(1-\frac{\omega_{p}^{2} / \omega^{2}}{1-\frac{n^{2} / \omega^{2}}{1-\omega_{p}^{2} / \omega^{2}}}\right)^{1 / 2} \simeq \frac{\omega}{c}\left(1-\frac{\omega_{p}^{2}}{2 \omega^{2}}\left(1+\frac{\Omega^{2}}{\omega^{2}}\right)\right),
$$

and

$$
k_{o}-k_{x} \simeq \frac{1}{2 c} \frac{\omega_{p}^{2} \Omega^{2}}{\omega^{3}} .
$$

Therefore, the phase difference is:

$$
\beta=\frac{1}{2 c} \frac{e^{4}}{\epsilon_{o} m^{3} \omega^{3}} n_{\mathrm{e}} B_{\perp}^{2} z .
$$

Limiting cases of Appleton's equation have indicated that the plasma induces an optical pathlength difference relative to a vacuum that depends primarily on density. The plasma also exhibits circular birefringence for $E M$ waves propigating along the magnetic field and linear birefringence for waves propagating in a transverse magnetic field.

Measurement of the effects a plasma has on $E M$ wave propagation can give information on the plasma density or magnetic field. These effects are averaged over the line of sight of the path of the beam. Techniques for inverting these chordaveraged measurements to obtain spatial profiles of the plasma parameters are the topic of the following section.

\subsection{Inverting Chord-Averaged Measurements}

There are many ways that chord-averaged data can be inverted to give information about the actual spatial profiles of the plasma parameters of interest. The techniques can be divided into two basic categories. In the first category, the spatial profiles are obtained by applying the Abel integral (or some expanded form of the iritegral) directly to the chord-averaged data taken along several chords in the 
plasma. In the second category, a spatial profile is predicted for the parameters of interest, and is tested for consistency compared to the chord-averaged measured values. The profile estimate is then adjusted until the chord averages of the profile adequately match the measured values. This method of inverting data is called forward optimization. The two categories of techniques obviously differ conceptually, but they also differ in accuracy and computational speed, as will be discussed below.

\subsubsection{Abel Inversion Methods}

Using A bel integrals to unfold chord-averaged measurements is described by Barr [56]. Figure 2.2 shows the geometry of the problem for a polnidal cross-section of the plasma. The chord integrated value of any scalar plasma parameter, e.g., plasma density, is given by:

$$
I(x)=\int_{-L / 2}^{L / 2} n_{e}(\vec{r}) d y
$$

A change of variables results in:

$$
I(x)=2 \int_{x}^{a} \frac{n_{e}(\vec{r}) r d r}{\left(r^{2}-x^{2}\right)^{1 / 2}}
$$

Equation 2.44 is one of Abel's integral equations. The full two-dimensional nature of the inversion proble makes it rather impractical (an.: sometimes impossible) to solve, because the problem is underdetermined in most experiments where only vertical access is available. For axial symmetry, the problem becomes one-dimentional, and can be readily solved. For $n_{e}(r)=0$ for $r>a$, this equation can be inverted to result in [57]:

$$
n_{e}(r)=-\frac{1}{\pi} \int_{r}^{a} \frac{(d I / d x) d x}{\left(x^{2}-r^{2}\right)^{1 / 2}} .
$$

For vector functions, the chord-integrated value of the plasma parameters needs to include the appropriate geometry. For Faraday rotation, the integrated value is given by:

$$
F=2.63 \times 10^{-25} \lambda^{2} \int_{-L / 2}^{L / 2} n_{e}(r) \vec{B}(r) \cdot \overrightarrow{d y} .
$$

Rewriting the dot product,

$$
F=K \int_{-L / 2}^{L / 2} n_{e}(r) B_{\theta}(r) \cos \theta d y
$$




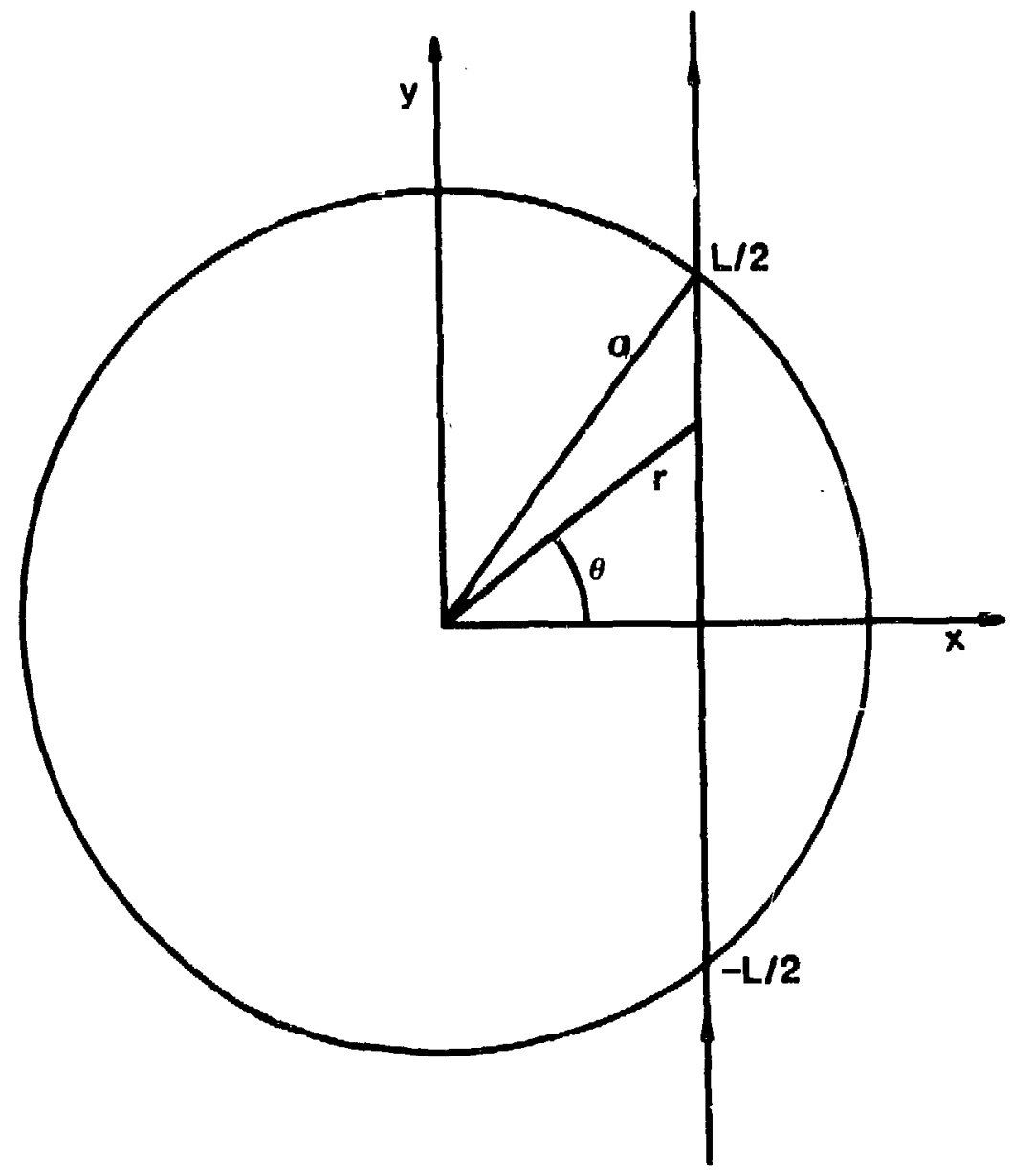

Figure 2.2: Poloidal Cross-Section Showing Geometry for Abel Inversion 
where $K=2.63 \times 10^{-25} \lambda^{2}$. Again, the result of changing the integration variable is:

$$
F(x)=2 K \int_{x}^{a} n_{e}(r) B_{\theta}(r) \frac{x d r}{\left(r^{2}-x^{2}\right)^{1 / 2}} .
$$

Taking an $x$ outside the integral, and defining a new function:

$$
G(x)=\frac{F(x)}{2 K x}=\int_{x_{x}}^{a} \frac{n_{e}(r) B_{\theta}(r)}{\left(r^{2}-x^{2}\right)^{1 / 2}} d r
$$

and defining a new variable in $r$ :

$$
\gamma(r)=\frac{n_{e}(r) B_{\theta}(r)}{r}
$$

then

$$
G(x)=\int_{x}^{a} \frac{\gamma(r) r d r}{\left(r^{2}-x^{2}\right)^{1 / 2}}
$$

Notice that $G(x)$ has the identical form of the Abel integral of Eq. 2.44, except the scalar plasma parameter is that which is defined in Eq. 2.50. This $G(x)$ can also be inverted, giving:

$$
\gamma(r)=-\frac{1}{\pi} \int_{r}^{a} \frac{(d G / d x) d x}{\left(x^{2}-r^{2}\right)^{1 / 2}},
$$

or the magnitude of the poloidal field is given by:

$$
B_{\theta}(r)=\frac{-r}{\pi n_{e}(r)} \int_{r}^{a} \frac{(d G / d x) d x}{\left(x^{2}-r^{2}\right)^{1 / 2}}
$$

where $G(x)$ is defined in Eq. 2.49. Therefore, the inversion of the Faraday rotation measurements can be made to look just like the inversion of the density measurement, or any other scalar. Extraction of the poloidal field information, of course, requires independent knowledge of the density profile.

Numerical solutions of Eq. 2.45 or Eq. 2.53 suffer from the fact that evaluation of the derivative amplifies the random noise present on any experimental measurement. Reference [58] presents an example where a 200-fold amplification of the noise results from the derivative cualuation. This is clearly enough of an effect to destroy what may be an otherwise useful measurement.

The standard technique for eliminating the sensitivity to noise amplification is to fit a smooth function to measured values $I(x)$ or $G(x)$ before performing the derivative. Barr [56] suggests a similar approach where the order of integration and 
differentiation is reversed from that implied by Eq. 2.45 or Eq. 2.53, and applying some smoothing techniques between operations to eliminate the noise amplification problem.

Analysis presented in Reference $[\mathbf{5 1}]$ reveals that use of this smoothed Abel inversion does have a drawback. The smoothed technique can lose some structure of the profile compared to the direct numerical inversion, especially when the number of chords is limited. Therefore, the sensitivity to noise amplification and the ability to adequately invert the measurement from a limited number of chords both enter into a decision on which inversion technique to use.

Deutsch and Beniaminy [58] have derived a derivative-free inversion formula that avoids the possible noise amplification complexity. Reference [59] outlines a method for allowing asymetries in the direction perpendicular to the direction of observation. This is accomplished by separating the function of measured values (e.g., $I(x))$ into odd and even parts and inverting these parts individually. Symmetry is still assumed for the direction along the observation, where no information on spatial variation is available.

\section{3.: Forward Optimization Methods for Inferring the Spa- tial Profiles}

The second basic method for unfolding chord-averaged data may be considered to be backwards in philosophy. It involves the adjustment of a hypothetical spatial profile until the resultant chord-integrated values adequateiy match the measured data. Typically, some functional dependencies of the spatial profile are assumed, and the coefficients of the functions are used as the fitting parameters (e.g., [60]).

An alternative method (e.g., [61]) that is numerically more intensive, involves calculating a family of profiles that would be consistent with the measured data. The ability to find several profiles that work is a consequence of the underdetermined nature of the problem. A so-called "minimum norm" criterion is used to determine the most probable profile of the family. This results in choosing the profile that has the shortest integrated arc length, or the least amount of structure on the profile. Figure 2.3 illustrates the minimum norm criterion, where profile $b$ would be chosen as being the more probable of the two rather extreme cases.

The forward optimization inversion technique has an advantage over standard Abel integral techniques in that there is no concern about derivative noise sensi- 

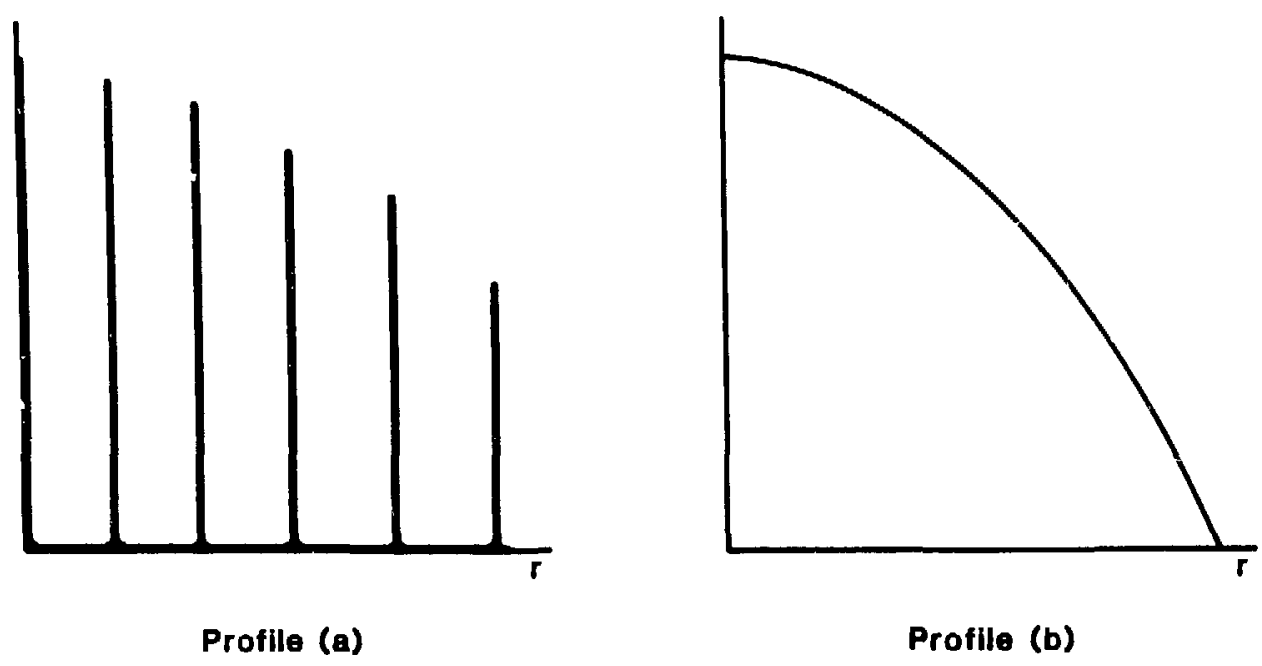

Figure 2.3: Two Possible Profiles for Minimum Norm Criterion 
tivity, error amplification, or other numerical pitfalls. This technique also gives quantitative information about the shape of the profiles through the final coeffcients of the functional description of th e profiles, giving an analytic expression for the profile. This might be useful for describing differences between profiles.

Because of the cumbersome iterative fitting routines, forward optimization methods are much slower than Abel inversion methods. Computational speed may be desired for determining the profile at many time points, or getting more immediate information about a plasma discharge. Therefore, both techniques for inverting chord-averaged data are useful, depending on the application.

\subsection{Error Propagation in the Inversion Process}

\subsubsection{Techniques used to Study Error Propagation}

1

In order to define the measurement accuracy required for adequate profile determination, analysis of how errors propagate in the inversion process is required. It was shown in the previous section that this error propagation depends somewhat on the inversion technique employed, because naive application of Abel's integral can result in a factor of $\sim 200$ amplification of the noise on the data.

There is a wide variety of sophistication that can be used for error propagation studies. Simple techniques can give quick qualitative results. However, more sophisticated techniques are required to accurately assign error bars to the final profiles. It is informative to consider the more sophisticated techniques first, and then explore which corners can be cut to give more expeditious answers.

The technique of Monte Carlo error analysis is described by Shirk and Hoffman [61) for $x$-ray spectral deconvolution applications, although it is flexible enough for inverting any scalar quantity. The method for performing the inversion was described in the previous section (2.3.2), where a family of possible profiles are calculated. The method is not prone to noise amplification effects, which would give overly pessimistic results. The Monte Carlo technique involves the following steps:

1. Estimation of the errors on the measured values for each channel or chord, usually based on signal-to-noise considerations. 
2. Selection of a set of "values" to be inverted. For each channel an artificial value is picked from a probabilistic distribution about the measured value. The distribution is gaussian with the width set equal to the experir atal error.

3. The set of values is inverted to give a possible profile within the experimental uncertainties.

4. Steps $1-3$ are repeated many times, until the statistical quantities are well established. A mean or average profile can be determined and a standard deviation calculated at each point in space from the entire set of inverted profiles.

Use of these steps generates quantitative infurmation about the error bars on the profile from uncertainties in the meas'ıred values.

\section{Simplified Error Analysis}

Information about error propagation can be obtained from less numerically intensive technique than the Monte Carlo method described above. Although rigorous error bar determination for the inverted profiles cannot be obtained from the simple techniques, useful information can be found concerning the measurement accuracy required for adequate profile determination. Thus, the simple techniques give guidelines to the experimentalist for system performance requirements.

The simplifications over the Monte Carlo method that will be used in the next section involve

a: running fewer cases $(\sim 10 \%)$ kecause the trends in the error propagation become apparent long before the Monte Carlo statistics are valid, and

b: using a single rectangular probability distribution for assigning errors to all of the artificial values, rather than using a Taussian distribution for each channel with the width corresponding to the measurement accuracy of that channel.

These simplifications allow useful answers to be found much more quickly. 


\subsubsection{Conclusions based on Simple Error Analysis}

Calculations were performed to try to get a feeling for how errors in measured values of chord-averaged density and Faraday rotation propagate through the inversion process. This exercise was initiated to give information on the measurement accuracy required in order to get useful density and poloidal field profile determination.

The level of sophistication of the inversion algorithm was intentionally kept low. The algorithm knows nothing of any information that might be available from other diagnostics. It uses a multi-parameter fit to the density and poloidal field profiles. The parameters are modified until the resulting profiles match the chord-averaged input data. This differs considerably in philosophy from standard Abel inversions, as was discussed in Section 2.3.1, where a functional fit of the measured values is performed, followed by application of the Abel integral to obtain the profile estimation. Abel inversions are numerically more efficient, but there is some potential numerical error amplification. Since efficiency was not a concern for the error propagation studies, the slower, but more straight-forward approach was chosen.

The input data, simulating measured values in the absence of actual multichord data, are introduced by assuming a profile and calculating the predicted chordaveraged values for each chord. Measurement errors are then introduced by a random selection of values in a range about the predicted value. The range is determined by the nominal experimental resolution achieved on ZT-40M, and the selection of any value in the range is equally probable. Typical performance of the interferometer results in a resolution of $\sim 0.01$ fringe. A phase resolution of $\sim 3 \mathrm{mrad}$ has been achieved by the polarimeter, although typical performance is probably a factor of two worse. (These figures correspond to $25 \mu \mathrm{sec}$ time resolution.)

The program then adjusts the parameters assumed for each profile until the inaccurate simulated values are least-squares fitted. The profile is plotted, and the program generates a new set of simulated measured values to be inverted.

The parametric description of the profiles invokes a shifted contour model. The basic contours can be thought of as nested circular surfaces, with the position of the centers of the contours being a function of radius. The shift of the magnetic axis is an input parameter, with the centers of the other contours following a linear 
function of radius, the outer contour having its center at the geometric axis. The profiles then have a functional dependence on contour radius. Even terms retain the circular shape of the contours and odd terms allow deformation of the shape from circular.

The functional form for the density profile is chosen to be:

$$
n_{e}(\rho)=P_{1}\left(1-\rho^{2} / a^{2}\right)+P_{2}\left(1-\rho^{2} / a^{2}\right)^{2}+P_{3}+P_{4} \cos \theta+P_{5}(1-\rho / a)
$$

The functional form for the poloidal field is taken to be:

$$
B_{\theta}(\rho)=Q_{1} J_{1}(15 \rho)+Q_{2} \cos \theta+Q_{3}+Q_{4}\left(1-\rho^{2} / a^{2}\right)+Q_{5}(1-\rho / a)
$$

where $a$ is the plasma radius, $\rho$ represents the radius of the contours, $\theta$ is the poloidal angle, and $P_{1-5}, Q_{1-5}$ are the adjustable parameters. The functional forms were somewhat arbitrarily chosen, although the fitting was quite flexible. (Different functional forms might be chosen for other applications.) The constant within the argument of the Bessel function was determined by defining the poloidal average reversal surface of the toroidal field to be at $\rho=.16 \mathrm{~m}$, and assuming that the arguments for the toroidal and poloidal Bessel functions should be the same. The initial parameters used for the calculations were $P_{1}=n_{e o}, P_{2-5}=0, Q_{1}=\frac{\mu_{o} I}{2 \pi a J_{1}(15 a)}$, and $Q_{2-5}=0$.

Interesting conclusions can be based on visual inspection of the results of the error propagation studies. Figure 2.4 shows the results of inverting 10 cases of measured values representing nominal ZT-40M parameters for a plasma current of $250 k A$. With the interferometer resolution set at .01 fringe and the polarimetric resolution set at $3 \mathrm{mrad}$, the determination of the density and poloidal field profiles seems to be adequate. Although this is not a statistically rigorous error bar determination, if ore were to invert actual measured data with the specified resolution, error bars corresponding to the width of the band of profiles drawn in each plot would be a reasonable approximation. For measured values representing $120 \mathrm{kA}$ operation of ZT-40M, however, the results are not as encouraging. Figure 2.5 shows the results of ten such cases. The density profile may be adequately determined, but the poloidal field profile has unacceptable error bars. Clearly, improvements would result if one were to impose boundary conditions on the poloidal field from external probe measurements. The error bars can be improved further with a sacrifice of time response. If the noise is truly random, the signal to noise depends 

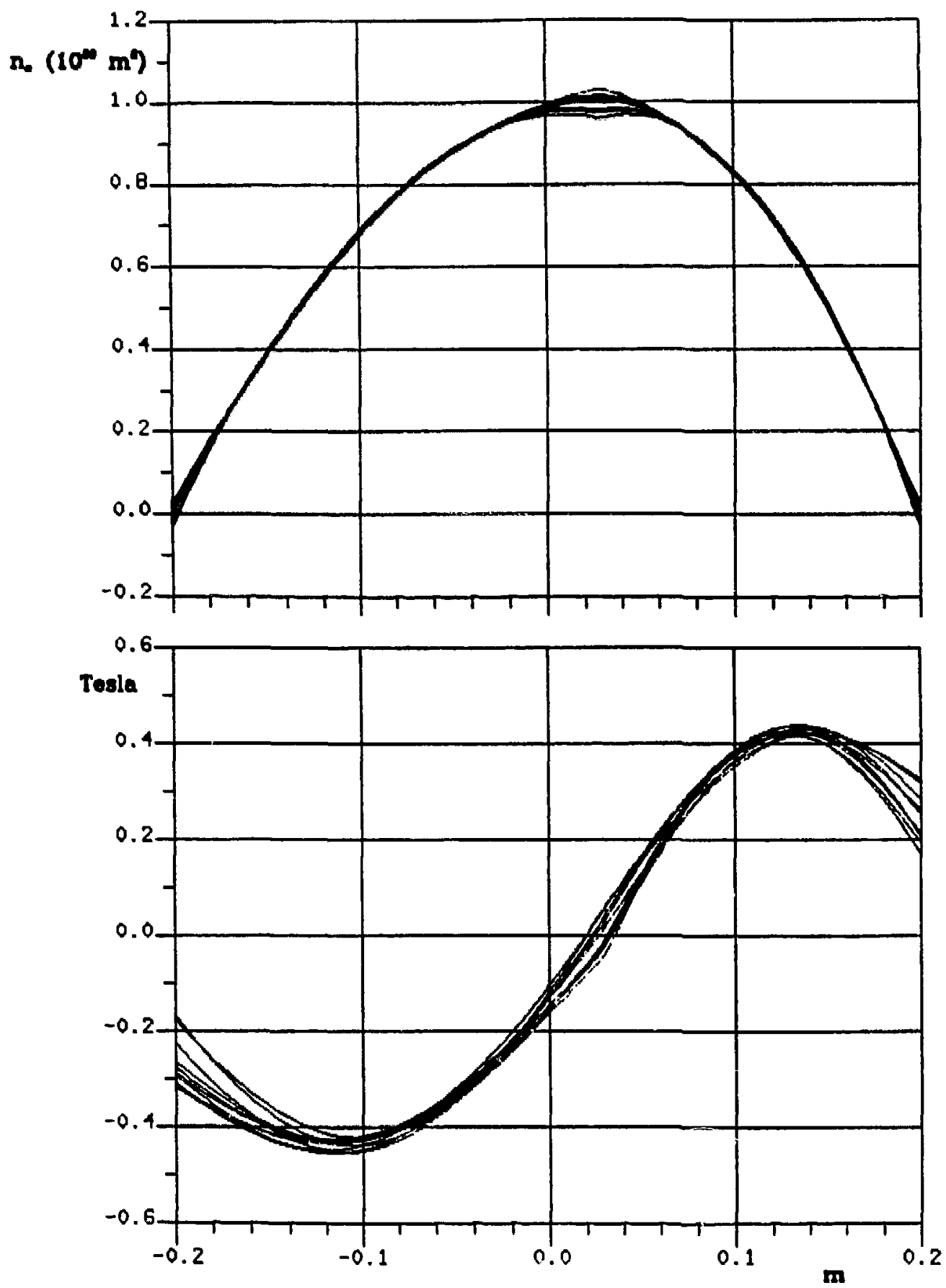

Figure 2.4: Density and Field Profiles for $250 k A$ Plasma, 3 mrad Resolution 

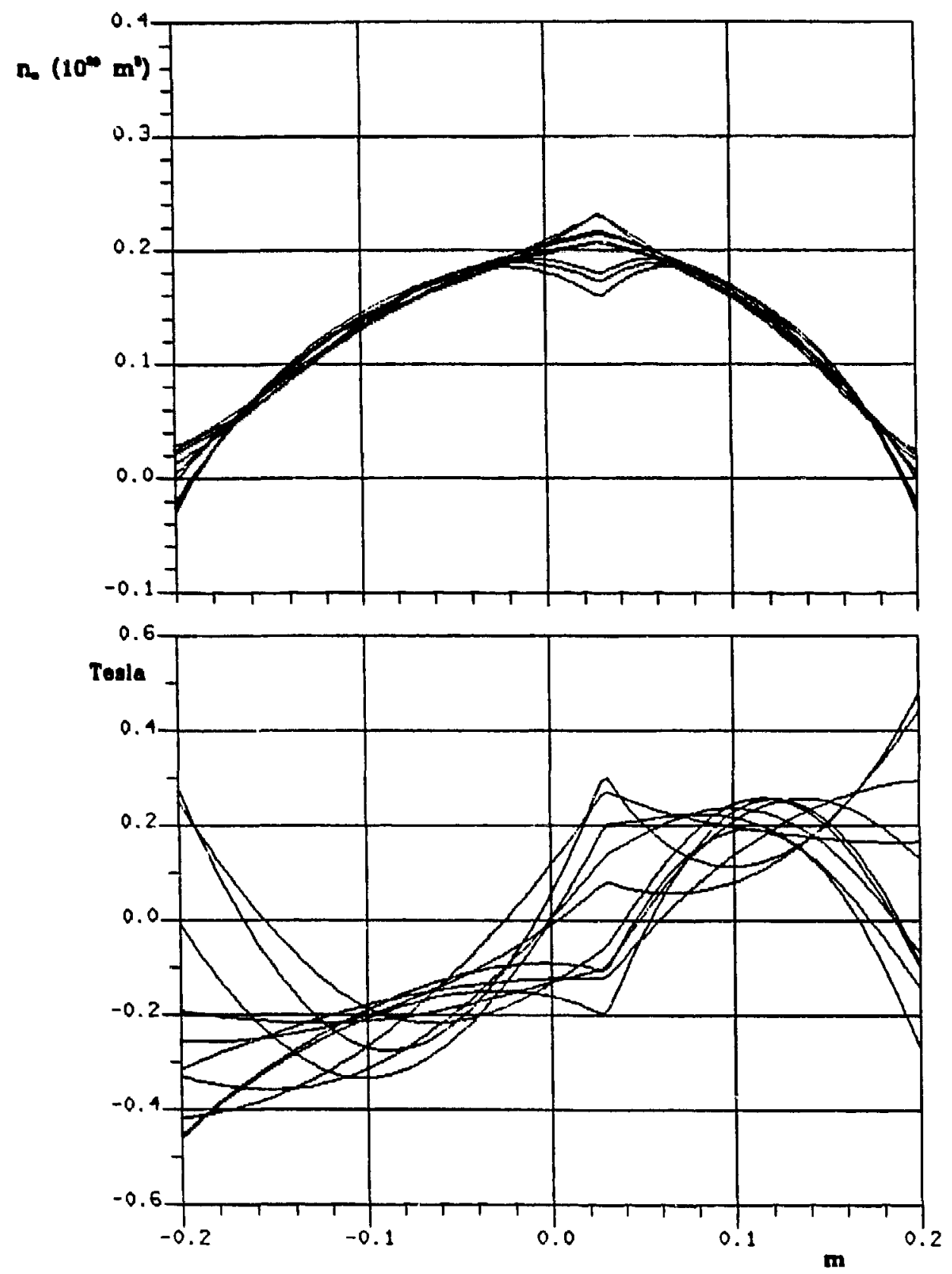

Figure 2.5: Density and Field Profiles for $120 k A$ Plasma, 3 mrad Resolution 


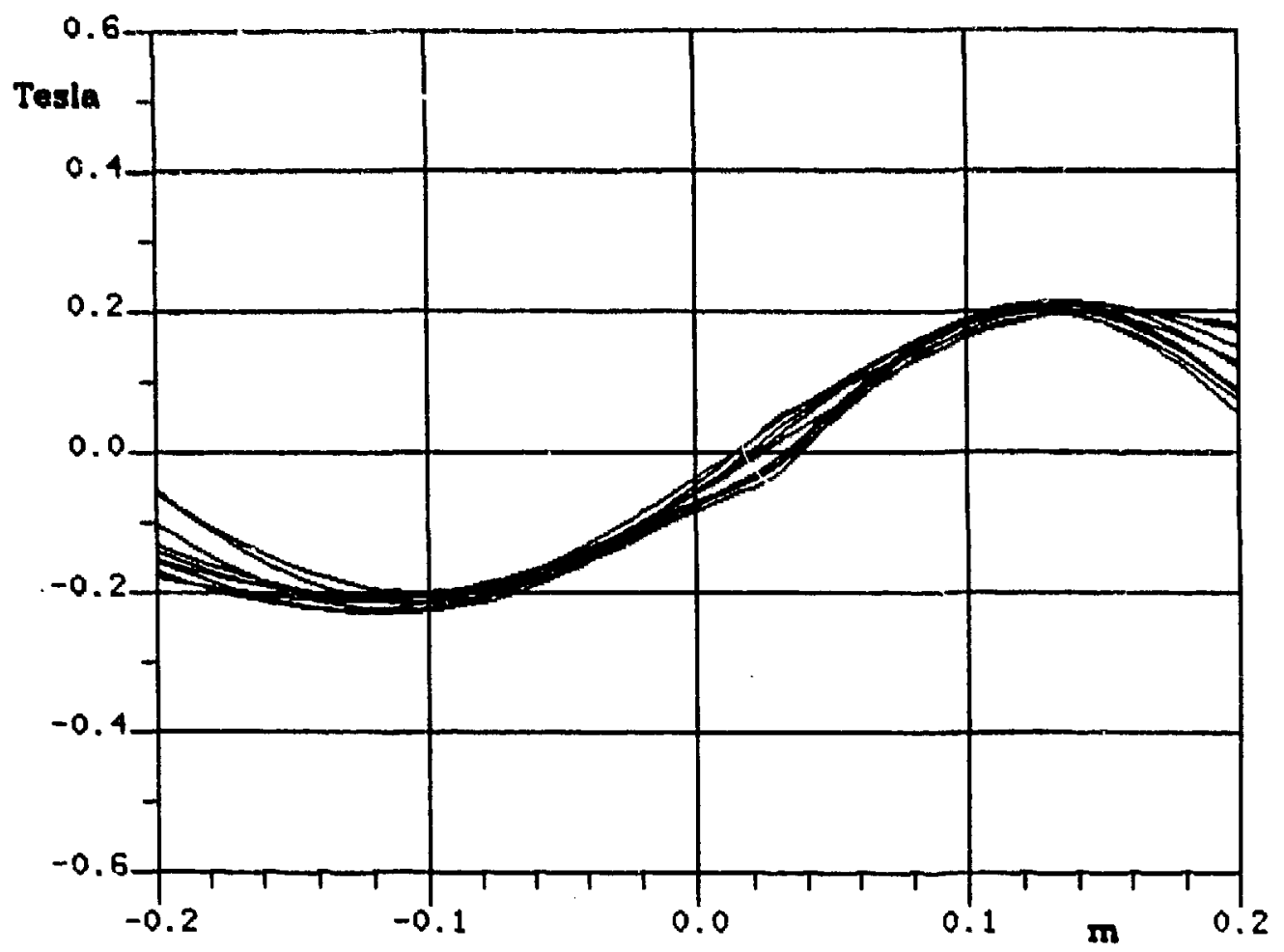

Figure 2.6: Poloidal Field Profile for $120 k A$ Plasma, 0.5 mrad Resolution

on $\sqrt{\tau}$, where $\tau$ is the integration time. If the time response is lengthened from $25 \mu s e c$ to $1 \mathrm{msec}$, the results are more acceptable. Figure 2.6 shows the results of improving the polarimetric resolution to $0.5 \mathrm{mrad}$, which would follow from the longer integration time.

It should be noted that eliminating the errors entirely on the density measurements did not significantly improve the error bars on the poloidal field profiles. Therefore, improvement in the interferometer sensitivity would not help the poloidal field profile determination. The dominant errors itı the poloidal field profile are the result of the polarimeter resolution.

The preceding calculations were done assuming 8 chords of data were being generated. The situation is somewhat degraded if only 6 chords of data are available. Integration times of at least $2.5 \mathrm{msec}$ were required to get adequate poloidal 
field profile determination for plasma parameters representing $120 \mathrm{kA}$ operation of ZT-40M.

\subsection{Conclusions}

The theory of $E M$ wave propagation in a magnetized plasma has been presented to form a basis for using $E M$ wave probing to measure the internal nagnetic field. The dominant effect of the plasma depends only on density, and methods for measuring the density through these effects will be discussed. Higher order effects depend also on the magnetic field. The plasma exhibits circular birefringence when the beam propagates parallel to the field (Faraday rotation) and linear birefringence when the beam propagates perpendicular to the field (the CottonMouton effect). Therefore, study of the polarization effects on the $E M$ wave probe can give information on the magnetic field. Although general polarimetry has not yet been developed, measurements can be made of either the circular birefringence or linear birefringence individually. The choice of $E M$ wave frequency can be made to cause either effect to be dominant. Particular attention has been paid to the measurement of circular birefringence, since it has the potential for internal magnetic field determination, and is the focus of the experimental part of this work. Techniques for inverting chord-averaged data were discussed, and analysis of how errors propagate in the inversion process revealed the experimental performance required to obtain adequate profile determination.

The following chapter is devoted to a review of techniques that have been developed and applied for the measurement of plasma parameters through effects described in Appleton's equation. Sources of errors or uncertainties in $E M$ wave probing will also be discussed. 


\section{Chapter 3}

\section{$E M$ Wave Probing Techniques}

The previous chapter described the plasma-uave interaction that forms the basis for the measurement of plasma parameters by $E M$ wave probing. This chapter will examine some of the $E M$ wave probing techniques that can be employed for a measurement of the plasma density and poloidal magnetic field. Measurement of the density is primarily made by interferometry, although there are several options on the type of interferometer used. For some applications, density measurements by $E M$ wave polarimetry might be preferable to interferometric techniques due to a greatly reduced sensitivity to mechanical vibrations. Field measurements can be made by $E M$ wave polarimetry, but again there are several options to consider.

All interferometers and polarimeters are subject to error. Some of the more advanced concepts are designed to virtually eliminate some sources of error, making them more useful for the measurements of interest. Detailed analysis of the potential errors will be presented below.

\subsection{Techniques for Measurement of Plasma Den- sity through $E M$ Wave Probing of the Plasma Refractive Index}

\subsubsection{Measurement of Plasma Density by $E M$ Wave Cut- off}

Ex mining Eq. 2.23 reveals that when the wave frequency is low enough, or the plasma density is high enough, the index of refraction becomes imaginary. In this case, the $E M$ wave is reflected rather than transmitted. The critical density where 
this transition occurs is given by the expression:

$$
n_{c}=\frac{\epsilon_{o} m \omega^{2}}{e^{2}}
$$

For typical densities of $10^{20} \mathrm{~m}^{-3}$, the wave frequency needs to be $\sim 90 \mathrm{GHz}$, or in the microwave region of the $E M$ wave spectrum.

This can be used to measure the density by measuring the frequency at which the transmission is interrupted, or when the reflected wave is detected. Simple techniques are limited by the range of frequencies that can be generated by a microwave source, and also provide information only about the peak density, whereas spatially resolved density profile measurements would be more useful. More sophisticated iechniques have been proposed [62], where the reflected wave is caused to interfere with the probe wave. Analysis can reveal how far the probe beam traveled in the plasma before it was reflected. This would allow a determination of the location of the cut-off surface, and give some information about the density profile.

\subsubsection{Measurement of Plasma Density by Interferometry}

\section{Basic Principles}

To measure the relative $E M$ wave phase change induced by the electron density of the plasma, interferometric techniques are often employed. ${ }^{1}$ Interferometry is the measurement of coherent interference (constructive or destructive) of the electric fields of two differt.tt waves. The two waves that are used for interferometric measurements of the refractive index are commonly called the scene and reference waves. The scene beam is propagated through the medium and is made to interfere with the reference beam that has bypassed the plasma.

The equation that describes the interference between two waves $E_{1}$ and $E_{2}$, of the same frequency and polarization, but with a phase difference $\phi$ between them is:

$$
E=\left(E_{1}+E_{2} e^{i \phi}\right) e^{-i \omega t}
$$

Detectors of the radiation generally sense the intensity of the wave rather than the electric field of the wave. The signal generated by such "square-law" detectors

\footnotetext{
${ }^{1} \mathrm{~A}$ useful review of interferometry is given by Jahoda and Sawyer[63], and by Véron[64].
} 
is then given by:

$$
E E^{*}=E_{1}^{2}+E_{2}^{2}+2 E_{1} E_{2} \cos \phi
$$

where the asterisk denotes the complex conjugate. The intensity at the detector has a constant component and a component which varies as the cosine of the phase difference between the waves. If, for example, the two waves have no phase difference in the absence of the plasma, then when a plasma is formed in the pathlength of the scene beam, the phase shift can be obtained through Eq. 3.2 ani is related to the density by (see Eq. 2.23):

$$
\phi=\frac{\omega}{c}(N-1) L \simeq \frac{\omega L}{2 c}\left(\frac{n_{e} e^{2}}{\epsilon_{o} m \omega^{2}}\right) .
$$

The WKB method allows the phase difference between the scene and reference beams generated by the plasma to be written as:

$$
\phi \simeq \frac{\omega}{2 c} \int_{L_{1}}^{L_{2}}\left(\frac{n_{\mathrm{e}} e^{2}}{\epsilon_{o} m \omega^{2}}\right)|\vec{d} l| .
$$

\section{Heterodyne Interferometry}

In practice, it is found useful to employ a slight frequency difference between the two beams of the interferometer. This technique is called heterodyne interferometry. The reason for the use of the two different frequencies is that the measurement of the phase difference induced by the plasma becomes insensitive to many effects that can cause ambiguity and error in the simpler methods. Most importanily, heterodyne methods eliminate the sensitivity of the density measurements to effects that cause spurious amplitude changes, such as refractive bending or partial absorption of the beam that propagates through the plasma or vignetting due to apertures that comprise diagnostic access.

Also, there is sometimes a condition that prevents complete destructive interference, which adversely effects the fringe ${ }^{2}$ conitrast or modulation depth of the interference pattern, and causes ambiguity in the simple amplitude measurements of a single-frequency interference pattern. Other disadvantages result from the details of the interference governed by Eq. 3.2, where it is seen that the phase shift

\footnotetext{
${ }^{2}$ The term fringe applies to a complete period of the sinusoidal intensity modulation generated by interference effects.
} 
direction is undetermined, and there are some phase angles where the measurement of phase changes becomes insensitive. All of these effects are avoided when heterodyne techniques are employed.

The interference between two waves of frequency $\omega_{1}$ and $\omega_{2}$ and phase difference $\phi$ is given by:

$$
E E^{\bullet}=E_{1}^{2}+E_{2}^{2}+E_{1} E_{2} \cos (\Delta \omega t+\phi)
$$

where $\Delta \omega=\omega_{1}-\omega_{2}$ is the difference frequency. Notice that there is still a constant component, but now the phase shift $\phi$ appears in a cosine term that varies in time with the difference frequency $\Delta \omega$, and the phase shift induced by the plasma density is seen on the detector as a phase shift of the sinusoidal time variation at the difference frequency. ${ }^{3}$ Therefore, amplitude changes that may result from propagation through the plasma do not affect the measurement of the density except for the smäll degree of coupling between amplitude modulations and phase modulations. For amplitude changes that are much slower than the difference frequency, this coupling is insignificant (see Eq. 3.23).

\section{Interferometer Configurations}

There are several configurations that are suitable for an interferometric measurement of the plasma density. The most common are the Michelson and MachZehnaer configurations. These are shown in heterodyne form in Figure 3.1 and Figure 3.2. The polarizing beam splitters (PBS) totally reflect one polarization and transmit the orthogonal polarization. The orthogonal polarizations have different frequencies, so the polarizing beam splitters actually perform the function of separating the two frequencies required for heterodyne interferometry. One frequency becomes the reference beam and the other becomes the scene beam. The non-polarizing beam splitters (NPBS) partially reflect and partially transmit both polarizations. The reflectivity of the NPBS is chosen to make the signal on each detector roughly the same level. Polarization analyzers (A) are placed in front of the detectors to act as "mixers" for the orthogonal polarizations. This function is required when square-law detectors are used because the two waves cannot interfere when their polarizations are orthogonal. The analyzers are similar to the polarizing beam splitters except for their orientation. They are oriented to transmit a

\footnotetext{
${ }^{3}$ This sinusoidal time variation is known as the carrier signal for the phase information.
} 


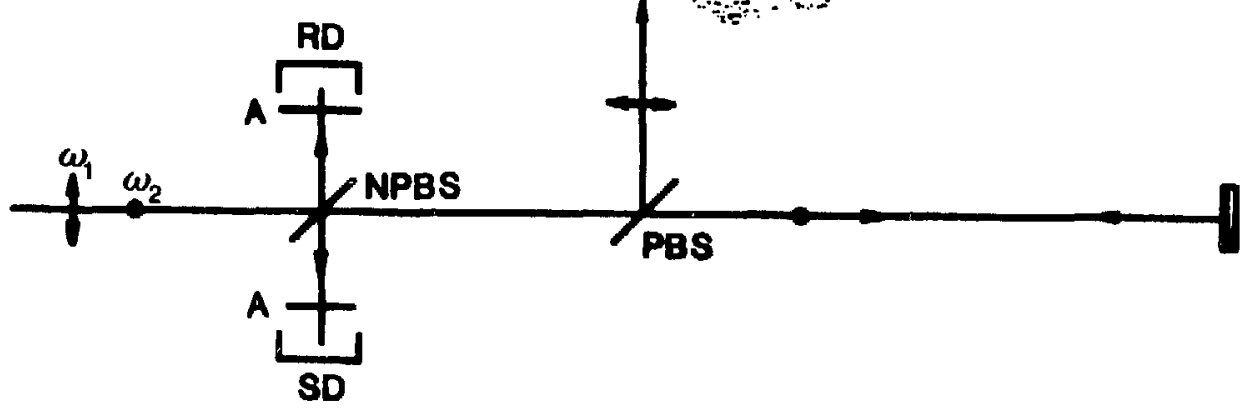

Figure 3.1: Michelson Configuration 


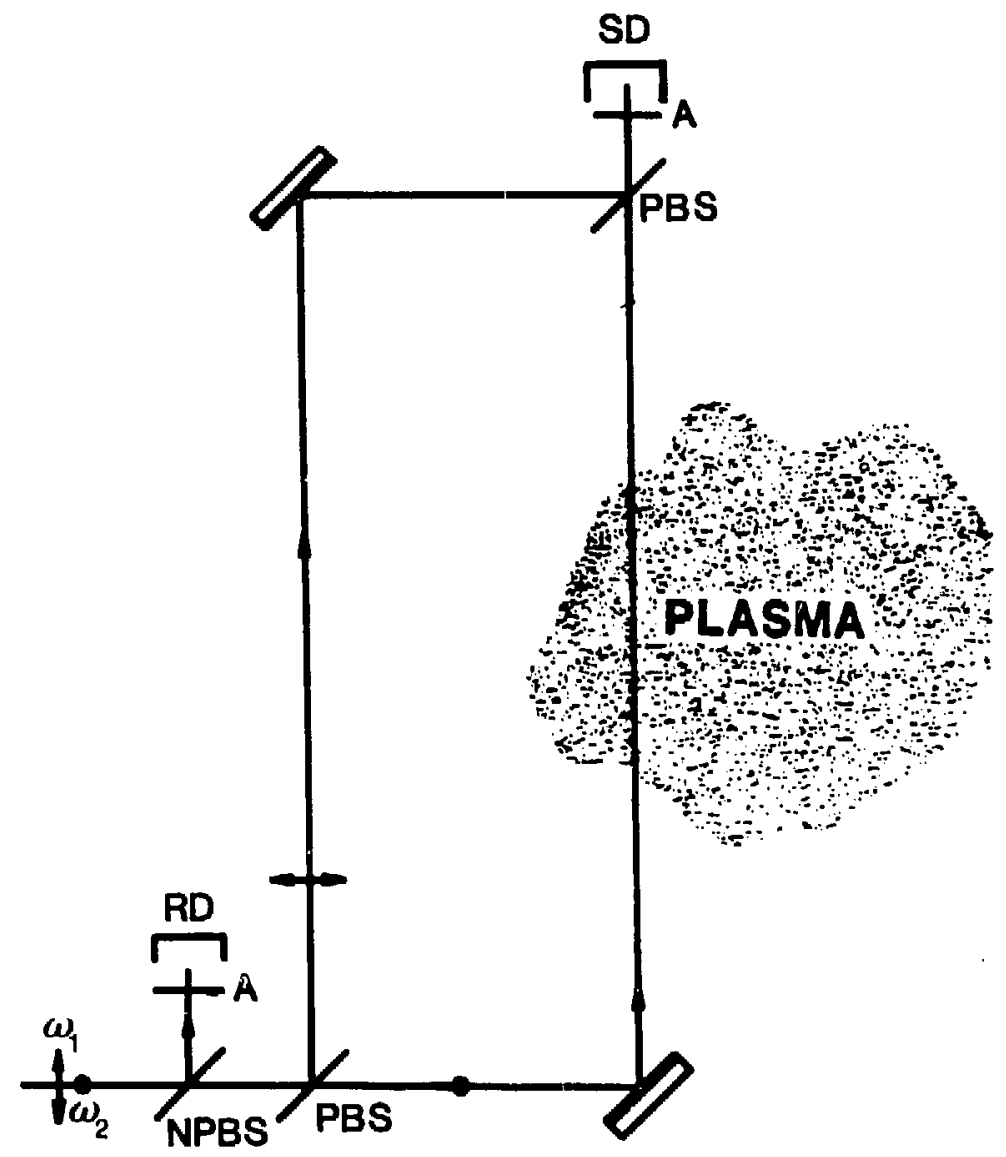

Figure 3.2: Mach-Zehnder Configuration 
component of each polarization (if the two beauns are of equal intensity, optimal orientation is at $45^{\circ}$ to the incident polarizations). The orthogonal polarizations are brought into the same plane of polarizatio by the analyzer, where they are now able to interfere. Some detectors sense the electric field rather than the intensity, and can perform the function of both the analyzer and detector shown in Figures 3.1 and 3.2 .

There are two detectors in each configuration. The signal detector (SD) records the heterodyne carrier signal which contains the plasma-induced phase shif: information, along with spurious information generated, in part, by drifts in the difference frequency. The reference detector (RD) records the heterodyne carrier signal which contains only the spurious information. Therefore, by determining the phase of the carrier signal recorded from both detectors, the plasma information can be extracted by taking the difference between the two phases.

The principle difference between the two interferometer configurations is that in the Michelson interferometer the scene beam makes a double pass through the plasma, which doubles the phase shift generated relative to the reference beam. This may be an important advantage if the expected phase shift is small, although it may be a disadvantage if the expected phase shift is large. The choice between the configurations is usually governed by other factors. Machine access constraints may require the use of one configuration. Use of the Michelson configuration allows almost all of the components of the interferometer to be on one side of the plasma, rather than having to surround the device with the Mach-Zehnder configuration. Use of the Michelson configuration also facilitates compensation for refractive bending, which may be severe enough to cause problems in overlapping the scene and reference beams on the detector. A retro-reflecting mirror (e.g., an internal corner of a metal cube or curved mirror) can be used to reflect the beam back through the plasma, sending it along nearly the same (possibly curved) path.

\subsubsection{Measurement of Plasma Density by Polarimetry}

The plasma density can be inforred by the measurement of ellipticity induced on an $E M$ wave, as long as the magnetic field of the plasma satisfies rather unique criteria. The plasma must have a transverse field that is much stronger than the parallel field (with respect to the wave propagation direction), and the transverse field must be spatially uniform. This is approximately true for a tokamak plasma 
(see Figure 1.1), and such a technique has been proposed by Craig [65] and by Segre [66].

If the poloidal magnetic field is neglected, and a linear polarization is injected into the plasma with a polarization azimuth at $\psi=45^{\circ}$ to the toroidal field (see Appendix B.1), then elliptization of the polarization will be induced. Examination of Equation 3.8 reveals that the ellipticity, $\beta$, depends only on $\int n_{e} d l$ if the transverse field dependence can be brought outside the integral. This is a reasonable assumption for a tokamak when the chords through the plasma are vertical. Since $B_{\phi} \propto 1 / R$, the toroidal field is independent of position along the beam.

For this technique to be reasonably accurate, the transverse field direction should be constant along the path of the beam, because the linearly birefringent character of the plasma vanishes if the polarization azimuth is aligned with or perpendicular to the magnetic field. Craig [65] shows that a $\cos 2 \psi$ term multiplies the $\int n_{e} d l$ factor in the ellipticity expression. Therefore, the polarization azimuth rotation must also be kept small, so that the angle $\psi$ does not change along the path of the beam. This requires the use of a relatively short wavelength to suppress Faraday rotation. As a result, however, the induced ellipticity is also small.

The attractive feature of $E M$ wave polarimetry for density measurements is the improved insensitivity to vibrations that can cause severe problems in interferometers. This feature is a result of eliminating the need for a reference beam that travels a different path, reflecting off different optics. In an interferometer, the character of the interference can be altered by vibration-induced optical pathlength changes that affect only one of the beams. The polarimeter can be considered to have two "beams" that travel the same path; therefore, vibrations affect both beams equally.

\subsection{Techniques for Measurement of the Internal Field via $E M$ Wave Probing}

Information about the internal magnetic field can be obtained through measurement of the polarization changes on an $E M$ wave probe imposed by the plasma. As was shown earlier, the plasma exhibits circular or linear birefringence depending on the orientation between the wave propagation vector, $\vec{k}$, and the magnetic field.

The expression found in Section 2.2 for $F$ and $\beta$ assumes that the plasma is uniform. As was done for the analysis of interferometry, the WKB method is used 
to allow for gradients resulting from the finite extent of the plasma, again with the condition that the gradient scale length be much longer than a wavelength. Applying the WKB approximation, and expressing the magnetic field in vector form rather than component form, the following expressions for $F$ and $\beta$ are obtained:

$$
F=2.63 \times 10^{-25} \lambda^{2} \int_{-L}^{+L} n_{e} \vec{B} \cdot \overrightarrow{d l}
$$

and

$$
\beta=2.44 \times\left. 10^{-29} \lambda^{3} \int_{-L}^{+L} n_{c}|\vec{B} \times \widehat{d}|\right|^{2} a i
$$

where $\lambda$ is in microns, $n_{e}$ in $m^{-3}, B$ in Tesla, $d l$ in meters, and $\widehat{d l}$ is a unit vector in the direction of $d l .^{4}$

Conceivably, if a measurement of the general state of polarization were made, the rotation of the azimuth caused by the Faraday effect would measure the parallel field and the elliptization caused by the Cotton-Mouton effect would measure the transverse magnetic field, provided there is independent knowledge of the plasma density. For the special case of a tokamak plasma, the parallel field can be found from the auimuth measurements, and density information can be obtained from ellipticity measurements, thus making polarimetry an alternative to interferometry for the measurement of plasma density (see Section 3.1.3). To date, the only polarimetric measurements that have been reported involve detecting the azimuth rotation caused by the Faraday effect. The ellipticity is not measured, per se, but ellipticity does have the potential of causing errors in the azimuth determination for some Faraday rotation measurement schemes. Polarimetric measurements have been made on vertical chords in a poloidal plane of toroidally shaped plasma, and therefore have given information about the poloidal magnetic field. Measurement of the toroidal magnetic field with the Faraday effect would be possible with the appropriate diagnostic access; however, such toroidal ports are not found on most machines.

\footnotetext{
'These expressions for $F$ and $\beta$ for oblique angles of propagation have some constraints. The Faraday rotation expression requires that the phase shift due to the Cotton-Mouton effect is small, and the Cotton-Mouton expression requires that the Faraday effect is small. For cases where these constraints are violated, the plasma has to be treated as an elliptically birefringent element (see Appendix B).
} 


\subsubsection{Amplitude Polarimetry}

Amplitude polarimetric techniques are somewhat analogous to the simple interferometric techniques described earlier, in that the azimuth rotation causes a change in the amplitude seen on the detector. A simple polarimeter appears in Figure 3.3, where a linear polarization is sent through the plasma. The polarization analyzer (A) is oriented to reject the radiation in the absence of plasma, and the detector (D) then sees a signal proportional to the sine squared of the rotation angle. The detection is facilitated by generating some form of amplitude modulation to enhance signal to noise ratios, because all but the modulation frequency can be discriminated against. One form of this intensity measurement technique is described by Soltwisch [40], and shown schematically in Figure 3.4. In this case, the probing wave, after passage through the plasma, reaches a polarizing beam splitter aligned to pass the original polarization and to reflect any orthogonal component induced by rotation. The reflected component combines with a frequency-shifted reference beam whose polarization is aligned to partially transmit through the beam splitter. The amplitude of the heterodyne beat is proportional to the plasma rotation. The complementary pair of beams in the orthogonal direction off the beam splitter is used for phase shift interferometry, which allows a simultaneous measurement of density and Faraday rotation to be made.

Soltwisch $[40]$ gives a comprehensive discussion of the considerable errors caused by spurious changes in the amplitude sensed by the detector. Refractive bending can reduce the amplitude of the signal due to vignetting by apertures and due to a change in the alignment of the beam relative to the detector, or the quality of beam overlap on the beam splitter. The amount of amplitude reduction could be calibrated with the change in carrier signal amplitude on the interferometer detector if the alignment changes affected both detectors equally. Soltwisch [40] observed that alignment changes affected the detectors differently; this was attributed to the different pathlengths from the plasma to the detectors. A second source of spurious amplitude is the polarizing beam splitter. Polarization elliptization causes a component to be reflected to the detector, which cannot be distinguished from azimuth effects. Elliptization can be caused by various system components as well as by the Cotton-Mouton effect in the plasma. These errors are discussed further in Section 3.3.2, along with a potential compensation for the contributions from polarization elliptization. 


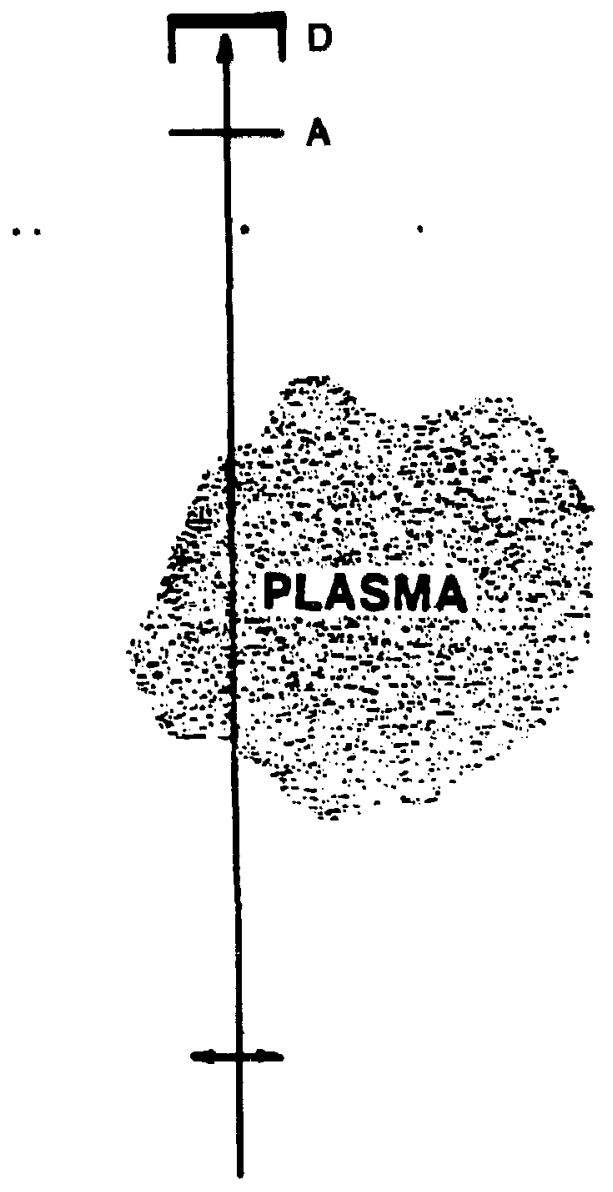

Figure 3.3: Simple Polarimeter 


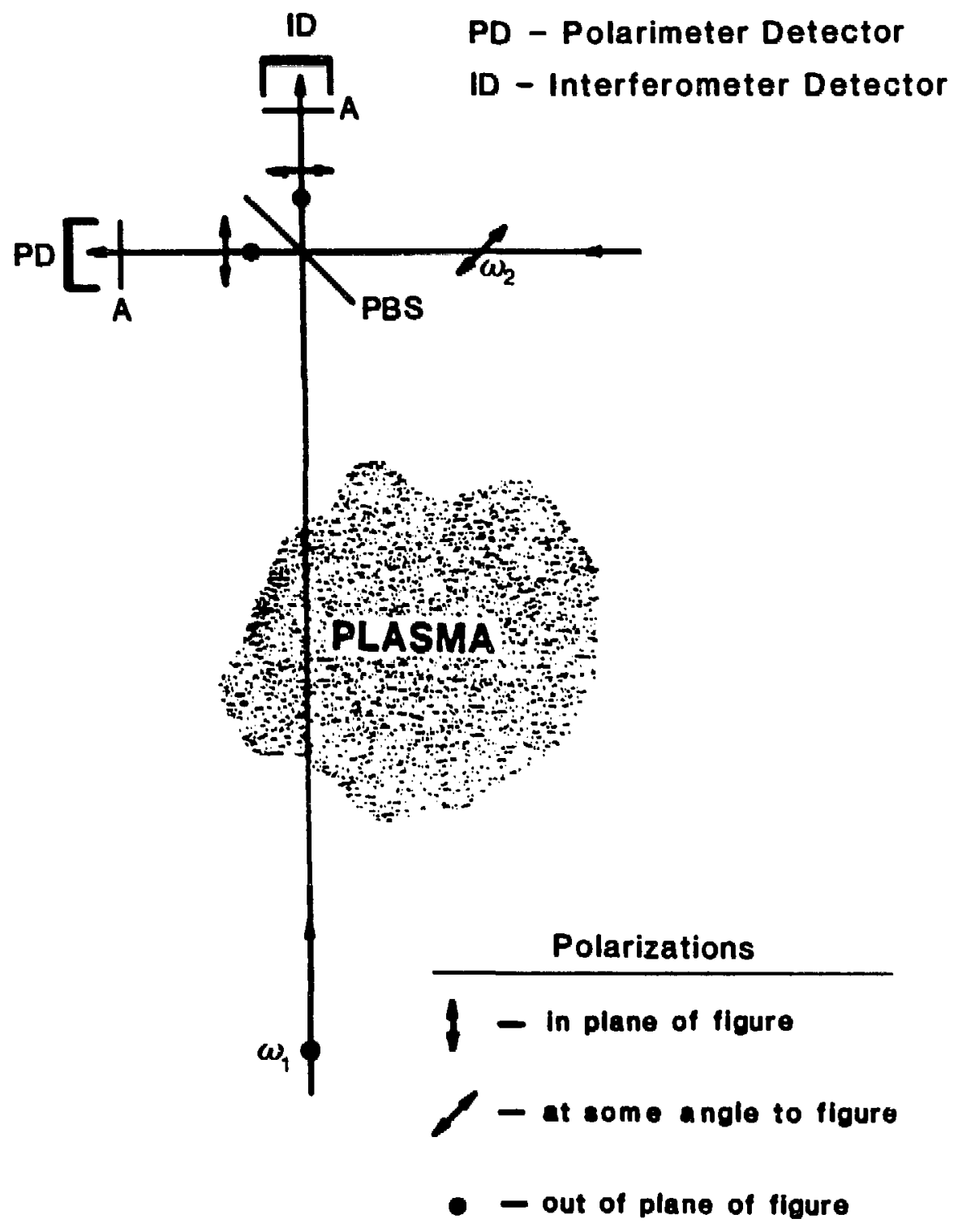

Figure 3.4: Soltwisch Configuration 
An alternative amplitude dependent measurement scheme, shown in Figure 3.5, introduces an angular oscillation of the input polarization (with a ferrite rotator) through small angles centered on the analyzer extinction direction. With no plasma, the detector senses the intensity variations at twice the modulation frequency. Faraday rotation induced by the plasma generates a signal at the modulation frequency. The amount of Faraday rotation is calculated from the amplitude of the signal at the modulation frequency $[42],[43]$. Because this method does not require two beams to be combined on a polarizing beam splitter, this method is inherently less sensitive to refractive effects. An initial drawback was the $10 \%$ transmission factor of the ferrite modulator $[42]$; however, development at Oak Ridge National Laboratory [43] resulted in a more acceptable transmission factor of $\sim 80 \%$. The higher transmission was obtained for $\lambda=447 \mu \mathrm{m}$ radiation. At shorter wavelengths, the transmission factor decreases rapidly, which limits the applications of this technique.

Heterodyne interferometry can be implemented with the ferrite rotator method, allowing a simultaneous measurement of density and Faraday rotation. This is accomplished by combining the scene beam with a reference beam (of a different frequency) before the detector. The density and Faraday rotation are measured with the same detector, which is possible if the difference in frequency between the scene and reference beams is much larger than the ferrite modulation frequency. Then, by filtering the signal appropriately for each frequency, both quantities can be recovered. This does, however, potentially limit the frequency response of the Faraday rotation measurement.

Just as was the case for simple interferometry, these amplitude polarimetry techniques are prone to uncertainties cause by spurious amplitude changes. Kunz [67] reported observations of $30 \%$ amplitude changes on TFR. Such errors are unacceptable for adequate determination of the poloidal field profile. Some additional (but less significant) errors will be discussed at the end of the next section, where a detailed formalism for error analysis is presented.

\subsubsection{Heterodyne Phase Shift Polarimetry}

A technique that is analogous to heterodyne interferometry is heterodyne phase shift polarimetry. This phase sensitive measurement is independent of absolute 


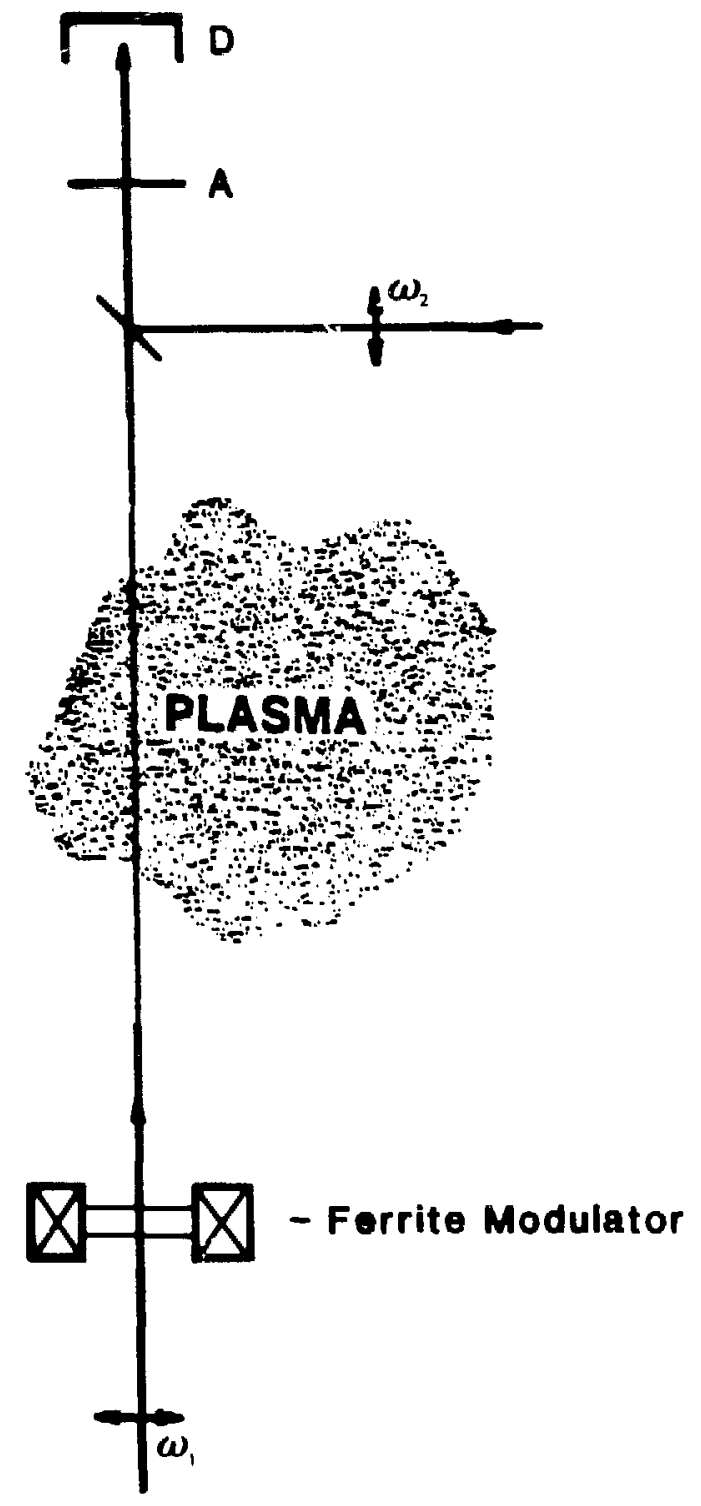

Figure 3.5: Ferrite Rotator Configuration 
amplitude, and insensitive to amplitude variations. This technique was proposed independently by Dodel and Kunz [48] and the diagnostic development group at Los Alamos [49]. The concept is illustrated in Figure 3.6. Opposite circular polarizations are given a frequency difference and sent through the plasma. These polarizations are produced by passing two orthogonal frequency-offset linear polarizations through a $\lambda / 4$ plate with the plate axes oriented at $\pm 45^{\circ}$ to the input polarization (see Appendix B.1). This condition is equivalent to having a linear polarization that rotates in time at half the difference frequency. In the absence of plasma, the signai seen by the detector (D) after the radiation passes through the polarization analyzer $(A)$ is an intensity that varies'sinusoidally in time at the difference frequency, or twice the frequency of the rotating linear polarization (this is due to the analyzer intensity transmission going through a whole cycle for a rotation of $180^{\circ}$ of the polarization). The Faraday rotation of linear polarization due to the circular birefringence exhibited by a magnetized plasma results from the speeding up and slowing down of the characteristic opposite circular polarizations into which the linear polarization can be decomposed (see Appendix B). Equivalently, for a rotating linear polarization (two circular polarizations of different frequencies), the Faraday effect will cause either a phase lead or phase lag in the rotation, depending on the direction of the magnetic field, and which circular polarization has the higher frequency. This produces a phase lead or lag on the sinusoidal temporal modulation of the detected signal in the presence of the plasma relative to the time signature in the absence of plasma.

Simultaneous interferometry measurements are also possible with this method. In this case, a second detector is required. This is accomplished by introducing a beamsplitter just beyond the plasma, and using one of the scene beam frequencies (say $\omega_{1}$ ) to combine with a reference beam of the other frequency $\left(\omega_{2}\right)$. This requires the other scene beam frequency (also $\omega_{2}$ ) to be filtered before the interferometer detector, which can be easily done (see Figure 4.15).

In common with the heterodyne amplitude method of Soltwisch [40], the time resolution is governed by the heterodyne frequency, which can be freely chosen. However, the heterodyne phase shift method differs in that departures from zero amplitude in the direction crossed to the analyzer not due io Faraday rotation, such as plasma- and component-induced ellipticities, do not directly add to the apparent plasma rotation signal. As mentioned before, the heterodyne phase shift 


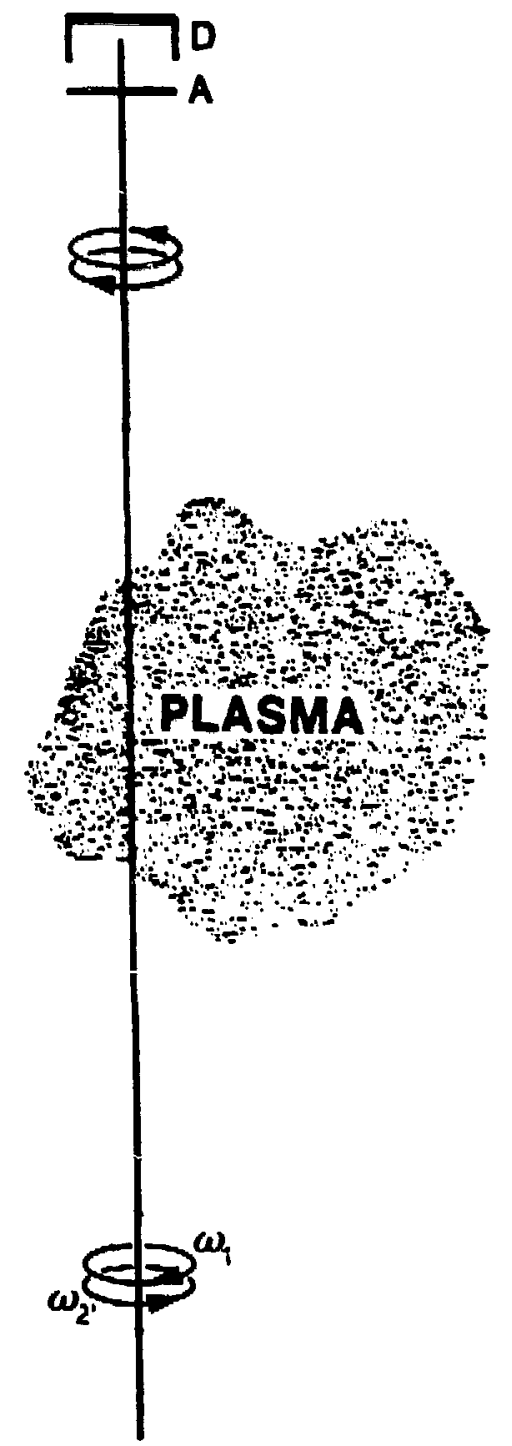

Figure 3.6: Heterodyne Polarimeter 
method is insensitive to spurious amplitude changes, which otherwise could cause very serious errors in the measurement.

Although heterodyne phase shift polarimetry is insensitive to the dominant errors of amplitude polarimetry, there are some sources of erroris that are common to all polarimetric magnetic field measurements. These will be discussed in the following section, where sources of errors in $E M$ wave probing techniques are presented.

\subsection{Sources of Errors in EM Wave Probing}

\subsubsection{Possible Sources of Errors in a Heterodyne Interfer- ometer}

Many of the potential errors in interferometric mieasurements are eliminated by using heterodyne techniques. As mentioned earlier, simple techniques are sensitive to spurious amplitude changes, ambiguity of phase change direction, insensitivity at certain phase angles, and errors introduced by imperfect interference of the scene and reference beams. The problems of insensitivity and directional arnbiguity at certain angles can be solved without heterodyne techniques by using quadrature methods [68], where both the sine and the cosine of the phase angles are measured; however, this method is still subject to amplitude problems unless heterodyne methods are also employed.

There are some problems that are common to all forms of interferometers. One problem occurs when large density changes take place on too fast a time scale. This could happen if a $\Delta \phi=2 \pi$ density change occurs between measurements, or on a timescale that is faster than the heterodyne frequency employed. This effect is called "fringe skipping," and can generally be avoided by using a measurement timescale, or heterodyne frequency, that is much faster than anticipated density changes.

Another source of error that causes problems for all interferometers is mechinical vibration. Although careful mechanical and optical design of the interferometer can minimize the sensitivity to vibrations, they can cause changes in the optical pathlength for either the scene or reference beam, leading to changes in the relative phase of the two beams when they interfere at the detector. The sensitivity to vibration is an inverse function of the wavelength employed, while the sensitivity 
to plasma density increases linearly with wavelen?t. Therefore consideration of vibration enters into the choice of wavelength for the measurements. There is usually a range of wavelengths that will have an acceptable insensitivity to vibration and not be overly sensitive to the refractive bending that scales with wavelength squared and becomes serious at longer wavelengths.

If such a range does not exist, however, a "two-color" technique is required [69]. There are two options of a two-color system that might be considered. The first system usis a relatively long wavelength that is sensitive to both the plasma density and vibrations. A very short wavelength beam is sent along the same path. This beam is rather insensitive to the plasma density (recall $N \sim \lambda^{2}$ ) and essentially measures only the vibrational information, which can be used to extract the density information from the long wavelength measurements. The second system uses wavelengths that are both sensitive to the plasma density and vibrations, but differ in wavelength so that the measurements can be used to solve a set of two algebraic equations with two unknowns: the plasma density, and vibration-induced phase shifts. This two-color method is, however, subject to the uncertainties of all amplitude techniques, unless the measurements can be made in heterodyne form. A heterodyne two-color interferorı eter has been developed by Mansfield, et al.[70], and used on the PLT tokamak.

Errors in the interpretation of the interferometric data can result from other plasma effects, such as ihe Faraday and Cotton-Mout n effects. These can usually be suppressed by careful choice of polarization; however, if polarimetric measurements are made along with the interferometric measurements, this flexibility may be limited. In particular, for interferometric measurements made simultaneously with heterodyne polarimetry (see Figure 4.15) the scene beam of the interferometer is circularly polarized. Because of the difference in plasma velocity for the two circular polarizations (which is the basis for the Faraday rotation induced by the plasma), the interferometric measurements will have slight systematic errors. The direction of the error depends upon which circular polarization is used for the scene beum. For nominal ZT-40M parameters, only a $0.3 \%$ error is introduced in the density measurements. For most plasmas of interest, the errors introduced by Faraday and Cotton-Mouton effects are generally very small.

Many sources of errors that are important to polarimetric measurement will be discussed later. For the most part, these errors also affect interferometric measure- 


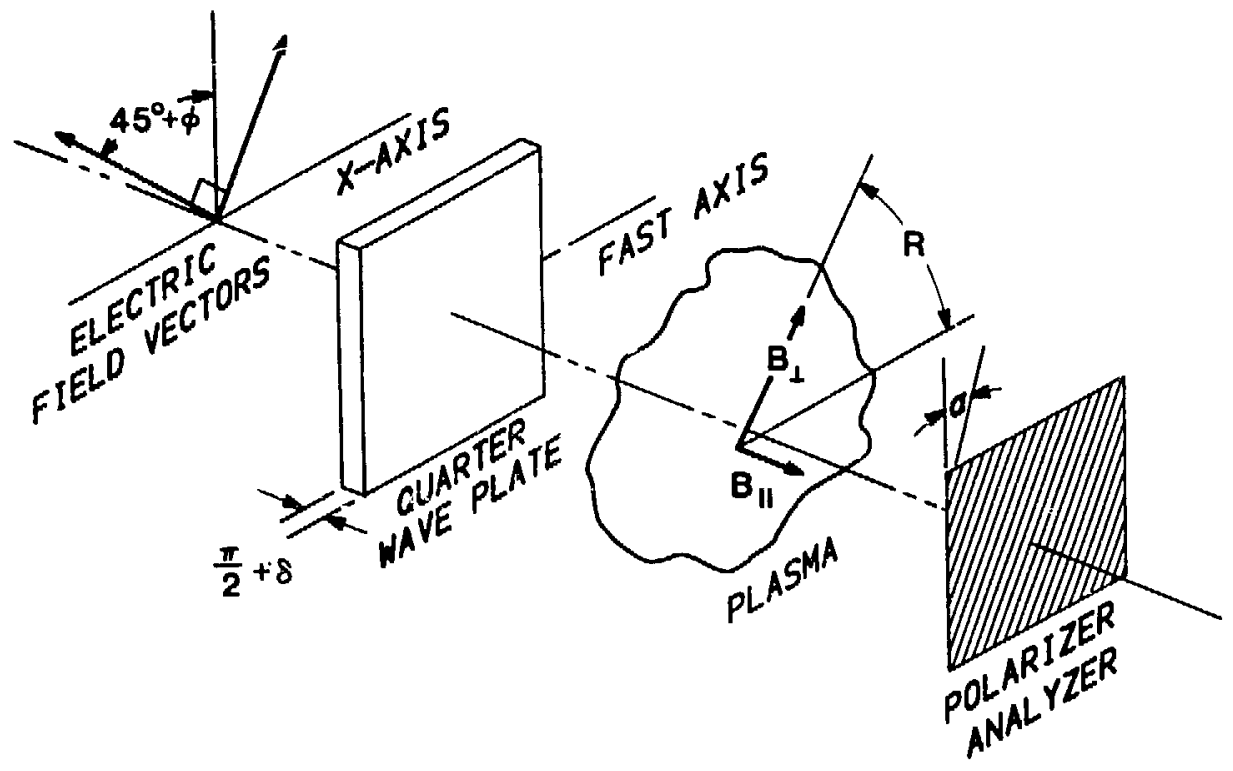

Figurs 3.7: Component System Used for Error Analysis

ments, but at such a low level that they are appropriately deferred to the following sections.

\subsubsection{Sources of Errors in a Heterodyne Polarimeter}

\section{Phase Shift Correction Factor}

To determine the possible errors in the heterodyne phase shift method caused both by linear birefringence due to transverse magnetic fields within the plasma and by optical misalignment or imperfections of the system coinponents, the intensity transmissi:.. through the system was analyzed, with the aid of Jones matrices (see Appendix B). This collaborative effort, motivated by the experimental work of this thesis, is reported in Reference[71]. The system to be analyzed appears schematically in Figure 3.7. The coordinate system is defined by the axes of the quarter wave plate. Experimental uncertainties include misalignment of the input 
radiation, thickness error, and dichroism of the $\lambda / 4$ plate, and polarization analyzer inefficiency and misalignment. Also considered are elliptization effects by the linear birefringence of the plasma. The basic procedure is to calculate the electric field components after transmission through the quarter wave plate, plasma, and linear polarization analyzer for one incident linear polarization and then for a frequency shifted orthogonal linear polarization. The detected intensity is given by the sum of the $x$ and $y$ components of the two frequencies. These steps are then repeated without the plasma and the phase shift difference of the intensity modulation with and without the plasma is examined as a function of the input parameters: plasma birefringence, angular misalignments, and optical component characteristics. In practice, it is useful to make analytical calculations for simple cases (e.g., one imperfection at a time), and to use a computer to perform the matrix operations for the more complicated cases.

Vector and Matrix Tofinitions The input electric field vectors for the calculations (see Figure 3.7) are:

$$
\left(\begin{array}{c}
\cos (\pi / 4+\varphi) \\
\sin (\pi / 4+\varphi)
\end{array}\right) \text { and } \quad\left(\begin{array}{c}
\cos (-\pi / 4+\varphi) \\
\sin (-\pi / 4+\varphi)
\end{array}\right) e^{i \omega t}
$$

why:re $\varphi$ is the angular input polarization misalignment from $45^{\circ}$ and $\omega$ is the radian frequency offset between the orthogonal polarizations. An amplitude difference be:ween the orthogonal inputs only affects the final intensity modulation. Since ti:is does not influence the phase shift, the amplitudes are assumed initially equal and normalized to unity.

The quarter wave plate matrix is given by (see Eq. B.9):

$$
T_{1}\left(\begin{array}{cc}
e^{i(\pi / 4+\delta / 2)} & 0 \\
0 & r e^{-i(\pi / 4+\delta / 2)}
\end{array}\right)
$$

where $\delta$ is the quarter wave plate thickness error in radians and $r=T_{2} / T_{1}$ is the relative amplitude transmission ratio of slow axis to fast axis. The latter is an expression of dichroismatic effects discussed in Appendix B in conjunction with the Poincaré sphere. 
The plasma matrix (see Eq. B.11) for a medium with circular and linear birefringence, can be written:

$$
\left(\begin{array}{cc}
\cos (\Theta / 2)-i \cos (\chi) \sin (\Theta / 2) & -\sin (\chi) \sin (\Theta / 2) \\
\sin (\chi) \cos (\Theta / 2) & \cos (\Theta / 2)+i \cos (\chi) \sin (\Theta / 2)
\end{array}\right),
$$

where $\Theta=\left(4 F^{2}+\beta^{3}\right)^{1 / 2}$ and $\chi=\tan ^{-1}(2 F / \beta)$. The optically activ. wunponent is expressed in terms of $2 F$ to be consistent with the earlier definition of $F$. As will be demonstrated, the pure Faraday rotation "phase shift" then becomes $2 F$. This is convenient in physical terms since a rotation of $180^{\circ}$ results in a complete cycle of intensity modulation as seen by the detector behind the polarization analyzer.

The plasma matrix as written iuplies a choice of coordinate system alignment with respect to the transverse magnetic field. To allow for arbitrary orientation of the transverse field in the coordinate system defined by the quarter wave plate, the plasma must be pre- and post-multiplied by the general rotation matrices given in Equation B.13. The polarization analyzer matrix is given by (see Equation B.12):

$$
\left(\begin{array}{ll}
1 & 0 \\
0 & 0
\end{array}\right)
$$

Ideal Case $(r=1, \varphi=\delta=\beta=R=\alpha=\epsilon=0)$ To illustrate the calculation prescription outlined at the beginni-so of this section, the ideal case is considered, where there are no misalignments or imperfections of optics nor any linear birefringence in the plasma. The result of multiplying the poiarizer matrix, plasma matrix, and $\lambda / 4$ plate matrix for this ideal case is:

$$
e^{i \pi / 4}\left(\begin{array}{cc}
\cos (F) & -i \sin (F) \\
0 & 0
\end{array}\right) \text {. }
$$

Next, each input electric field (see Equation 3.9) is multiplied by this matrix separately. Addition of the $x$ and $y$ components of each final electric field vector results in:

$$
\left(\begin{array}{c}
E_{x} \\
E_{\nu}
\end{array}\right)=e^{i \pi / 4}\left(\begin{array}{c}
\cos (F)\left(1+e^{i \omega t}\right)-i \sin (F)\left(1-e^{i \omega t}\right) \\
0
\end{array}\right) .
$$

The intensity seen on the detector is found by multiplying each component $\mathrm{bj}^{\mathrm{j}}$ its complex conjugate:

$$
I_{p}=E_{x} E_{x}^{*}+E_{y} E_{y}^{*}=2[1+\cos (\omega t+2 F)] .
$$


The same procedure is done without the plasma matrix resulting in:

$$
I_{o}=2[1+\cos (\omega t)] \text {. }
$$

Note that both $I_{0}$ and $I_{\mathrm{p}}$ have a constant component and a component that modulates in time with period $T=2 \pi / \omega$, but $I_{p}$ leads $I_{o}$ in phase by $2 F$.

Quarter Wave Plate Thickness Error Case $(r=1, \phi=\beta=R=\alpha=\epsilon=$ $0, \delta \neq 0$ ) The result of multiplying the polarizer matrix, plasma matrix, and $\lambda / 4$ matrix for this case where only one imperfection is considered is:

$$
e^{i \pi / 4}\left(\begin{array}{cc}
e^{i 5 / 2} \cos F & -i e^{-i \delta / 2} \sin F \\
0 & 0
\end{array}\right) .
$$

Repeating the same steps outlined in the ideal case results in:

$$
I_{p}=2[1+\cos (2 F) \cos (\omega t)-\sin (2 F) \sin (\omega t) \cos \delta\},
$$

and

$$
I_{0}=2[1+\cos (\omega t)],
$$

as before. To proceed further in identifying the error introduced on the apparent phase shift, a small angle approximation is made for the phase shift, i.e., $\cos 2 F \simeq 1$ and $\sin 2 F \simeq 2 F$. Eq. 3.18 then simplifies to:

$$
I_{p}=2[1+\cos (\omega t+2 F \operatorname{ros} \delta)] .
$$

Thus a multiplicative error of $\cos \delta$ over the phase shift for the ideal case results from a thickness error $\delta$ on the quarter wave plate. The small $2 F$ approximation makes less than $1 \%$ errors for $2 F \leq 150$ mrad.

Correction Factor for More General Case $(R=\alpha=\epsilon=0, \beta, \varphi, \delta, \neq 0, r \neq 1)$ Proceeding in a similar manner for variations in the parameters $\beta, \varphi$, and $r$, the following result is obtained (recall $\left.\Theta \equiv\left(4 F^{2}+\beta^{2}\right)^{1 / 2}\right)$ :

$$
\text { Phase Shift }=2 F\left[\frac{\sin \Theta}{\Theta} \cdot \frac{1}{\cos (2 \varphi)} \cdot \cos \delta \cdot r\right]_{R=0},
$$

where it has been assumed that the total phase shift is much less than the unity. In the ideal limit, the correction factor of the phase shift becomes $\sin (2 F) /(2 F)$. 
For small $F$, where the correction factor is valid, it is essentially unity. The range of validity of the analytical form as the factors depart increasingly from one is more readily explored by computer code. A non-unity correction factor implies the need for a (one-time) calibration to relate phase shift measured to the line integral $\int n_{8} \vec{B} \cdot \overrightarrow{d l}$. Once the calibration is established, the proportionality is completely determined by physical constants.

\section{Effects Implied by the Correction Factor}

Effects of Plasma Linear Birefringence The previous analysis has neglected any additional effects that might result when the transverse magnetic field is not aligned with the coordinate system. This is of little consequence for the parameters presently of interest, where the $\Theta$ dependence $\left[\Theta=\left(4 F^{2}+\beta^{2}\right)^{1 / 2}\right]$ is essentially negligible, since $\beta \ll 1$ and $F \ll 1$. To obtain the dependence in the more general case of $R \neq 0$, the orientation effects can be analyzed.

Repeating the calculations of the Jones matrix formulation, the phase shift for $\alpha=\varphi=\epsilon=0, r=1, \beta \neq 0, R \neq 0$ is calculated to be:

$$
\text { Phase Shift }=2 F-\left(\beta^{2} / 4\right) \cos (2 R) \sin (2 R),
$$

where it has again been assumed that $\beta \ll 1$ and $F \ll 1$ so that the approximation $\sin \Theta \simeq \Theta$ is valid. Equation 3.22 illustrates the fact that if $\beta^{2} / 4 \ll 2 F$, care should be taken to align the coordinate system as best as is possible with respect to the direction of the transverse magnetic field to minimize the effects of plasma linear birefringence.

Effects other than Plasma Birefringence The analytic computation for errors in heterodyne phase shift polarimetry has been based on a coordinate system orientation referenced to the quarter wave plate. The detector is behind a polarization analyzer that ideally must be aligned along an axis of this coordinate system $(\alpha=0)$ because the errors explicitly considered all introduce asymmetries in the orthogonal components of this coordinate system. However, as readily verified by the computer program, the absolite values of the error corrections are rather insensitive to a :elatively large error (e.g., $\alpha \leq 10^{\circ}$ ) in the actual orientation of the analyzer. A similar argument applies to show that the extinction factor $(\epsilon \neq 0)$ of the polarizer is not critical. This is quite different from the amplitude polarimetric 
techniques, where the analyzer defines the coordinate system orientation and, in order to achieve a null for zero rotation, must be precisely aligned to the input radiation coordinate system and have a very large extinction ratio.

Ellipticities introduced by optical components such as windov's and beam splitters enter into the amplitude factor of the signal, but do not affect the observed phase shifts as long as they remain constant throughout the plasma discharge. This is intuitively obvious since a rotating ellipse will only reduce the amplitude of the modulation relative to a rotating linear polarization.

Beam wandering on the optical components due to plasma refractive bending can cause significant amplitude variation, especially if the beam becomes only partially coupled to the detector, or vignetting is caused by the diagnostic access. This will influence the phase shift measurement only momentarily by a very small correction to the phase during the amplitude change through amplitude modulation (AM) to phase modulation (PM) coupling. This coupling depends on the percent amplitude charıge and the AM frequency as one would intuitively guess:

$$
\text { Phase Error } \propto \% A M \frac{\omega_{A M}}{\omega_{H}},
$$

where $\omega_{A M}$ is the AM angular frequency and $\omega_{H}$ is the heterodyne frequency. Therefore, by choosing a higher heterodyne frequency this momentary phase error can be made arbitrarily small. This is in striking contzast to the amplitude polarimetric techniques which are directly sensitive to amplitude efiects, not only during the amplitude charige, but for the entire duration of the amplitude reduction caused by refractive bending. This obviously may be the case for the entire plasma discharge.

In common with all methods, the heterodyne phase shift method is ultimately limited by signai to noise considerations of the modulated carrier, which in this case carries the phase shift information. For correction factors on the phase shift which are near unity, the amplitude decrement of the carrier signal will be negligible. However, for correction factors that depart significantly from unity, consideration of the carrier amplitude becomes important as the noise on the carrier may become more significant. 


\section{Effects of Linear Birefringence on Amplitude Polarimetry Method}

For comparison, the rocking polarization method (see Figure 3.5) and the heterodyne amplitude method (see Figure 3.4) can be analyzed in a similar fashion. For the rocking polarization method, the single incident electric field column vector is:

$$
E_{o}\left(\begin{array}{c}
\cos (A \cos (\Omega t)) \\
\sin (A \cos (\Omega t))
\end{array}\right)
$$

and the analyzer matrix is:

$$
\left(\begin{array}{ll}
0 & 0 \\
0 & 1
\end{array}\right)
$$

where $\Omega$ is the frequency at which the polarization is rocked, and $A$ is the amplitude of the rocking expressed in radians. The rroblem is made relatively easy by the fact that only the terms containing $\cos (\Omega t)$ need be kept (there are no $\sin (\Omega t)$ terms). The result after applying the analogous procedure is:

$$
I_{\Omega}(A)=4 E_{0}^{2} G(A)\left[2 F-\left(\beta^{2} / 4\right) \cos (2 R) \sin (2 R)\right] \cos (\Omega t),
$$

with

$$
G(A)=J_{o}(A) J_{1}(A)+\sum_{k=1}^{\infty} J_{2 k}(A)\left[J_{k+1}(A)-J_{k-1}(A)\right],
$$

where $J_{k}$ are Bessel functions. This exhibits the identical $R$ dependence, i.e., the same care should be taken in aligning the polarizer with the transverse field in this case. The optimum value for $A$ is $0.92 \mathrm{rad}$. At this value, the signal is greatest and there is a minimum change in the signal for a given change in $A$. Values of $A$ that have been used in polarimetric systems are $.05 \mathrm{rad}$ by [67] and $.42 \mathrm{rad}$ by [43].

For the heterodyne amplitude met. ${ }^{2} i$, it can be shown that the component of the signal oscillating at the beat frequency can be expressed as:

$$
\text { Signal } \sim E_{1} E_{2}[F \cos (\omega t)+\beta \cos (R) \sin (R) \sin (\omega t)] .
$$

The amplitude of the signal is obtained by use of a lock-in amplifier [40]. The signal is mixed with a reference signal $E_{3} \sim \cos (\omega t+\Psi)$ which is presumed to have a small phase error $\Psi$. The resultant signal is then:

$$
\mathrm{S}_{\text {Lork-in }} \sim 2 F^{\prime} E_{1} E_{2} E_{3}[\cos (\Psi)-(\beta / 2 F) \cos (R) \sin (R) \sin (\Psi)] \text {. }
$$

In addition to dependence on angular adjustment through $R$, the sensitivity to linear birefringence depends on how accurately the phase of the reference signal 
can be set. By simultaneously taking a quadrature of the signal, correction for elliptization of the signal beam should be possible, and allow both Faraday rotation and Cotton-Mouton effects to be measured simultaneously.

\section{Phase Shift Enhancement}

The theoretical discovery and experimental verification of a phase shift enhancement was first reported by the diagnostic development group at Los Alamos in Reference $[71 \mid$, along with an abbreviated account of the error analysis presented above. There are two factors in the correction factor of Eq. 3.21 that can be deliberately made much greater than 1 . They will be discussed separately, but there is no reason in principle why they cannot be invoked simultaneously, each contributing a portion to the desired total phase shift gain. The penalty paid for this increased phase shift is the amplitude decrement of the signal carrying the phase shift information. For one enhancement scheme, the amplitude is decreased by the enhancement factor, and by the square of the enhancement factor for the other scheme. As the individual factors get larger, non-linearities ignored in the analytic result will become significant, and it is best to be guided by numerical computation. In particular, because each factor is linked somewhat differently to the transverse coordinate frame, and thereby interlinked differently to the polarization analyzer orientation, the simultaneous effects cannot be expected to be simply multiplicative. Nonetheless, the analytic expression is a good guide for getting more than an order of magnitude enhancement from either factor sieparately or from a combination.

Enhancement Through Errors in the Input Polarization Angle The $1 / \cos 2 \varphi$ term in Eq. 3.21 can be made intentionally greatẹ than one. Theoretically, to get a factor of 10 requires $\varphi=42^{\circ}$, i.e., the input polarization, instead of being $\pm 45^{\circ}$ from the quarter wave plate axes, must be displaced only $3^{\circ}$ from these axes. The computer program result for $\varphi=42^{\circ}$ and for polarization analyzer setting $\alpha=0^{\circ}$ gives a phase shift of $y .5^{\circ}$ and a carrier amplitude decrement of 9.4 for a $2 F$ value of $1^{\circ}$. Note that there are nonlinearities associated with the value of Faraday rotation $F$ also, and therefore these calculations are restricted to the practical situation of small $F$. Sensitivity to the analyzer setting is much increased for such an extreme input orientation misalignment. For instance, the phase shift is 

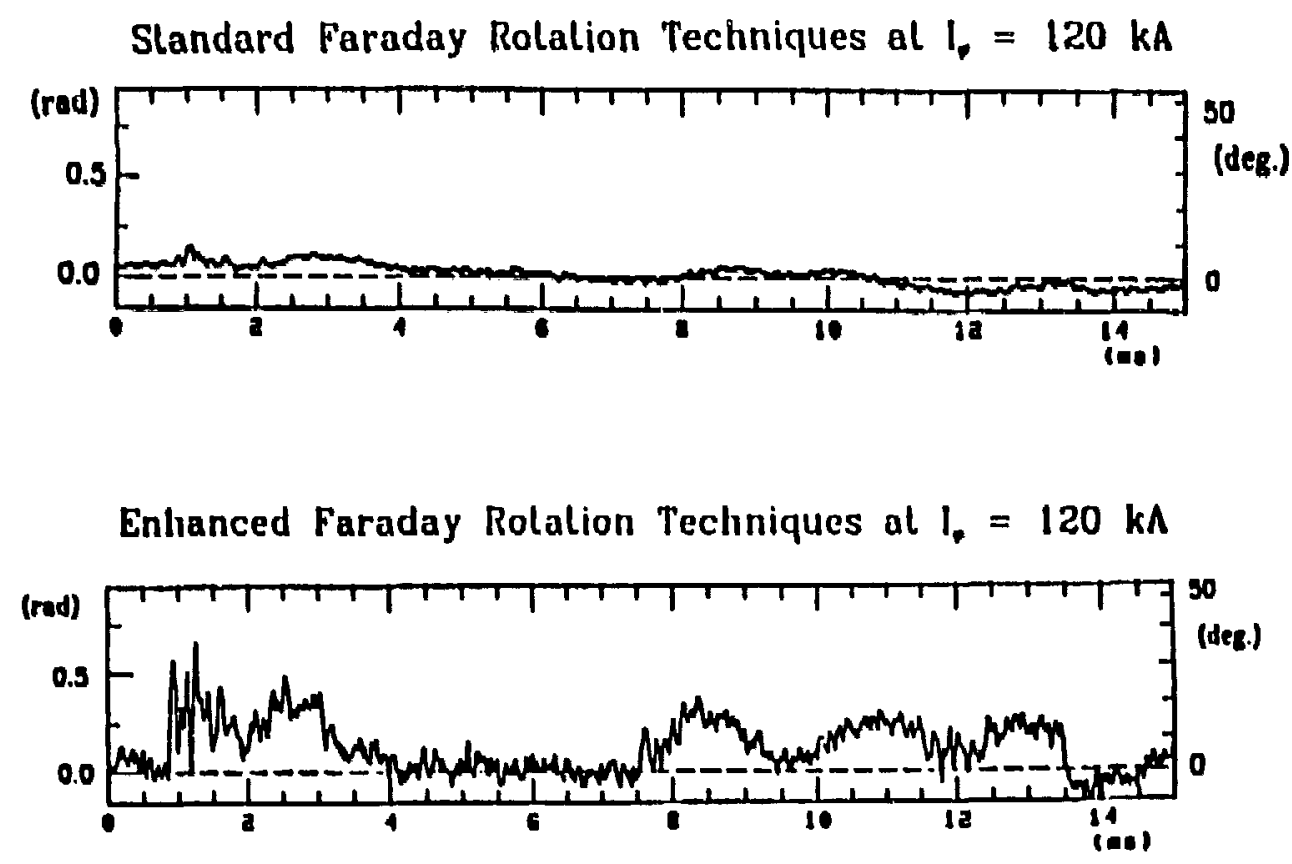

Figure 3.8: Example of Faraday Rotation Enhancement

$5.3^{\circ}$ and the amplitude decrement is 7.4 for $\alpha=3^{\circ}$, and the phase shift is $4.4^{\circ}$ and amplitude decrement 6.3 for $\alpha=-3^{\circ}$ (note the asymmetries), both for $2 F=1^{\circ}$.

The qualitative experimental confirmation of these predictions is shown in Figure 3.8, for two nominally identical plasma discharges on ZT-40M, where the enhancement was achieved by setting the $\lambda / 4$ plate at $\varphi=42^{\circ}$. At the time these measurements were made, the small true Faraday rotation signal was masked by extraneous phase shifts due to problems that will be discussed later at great length. Therefore, no physical interpretation of plasma characteristics should be drawn from this data; however, the demonstration of phase shift enhancement remains valid.

Enhancement through Quarter Wave Plate Dichroism The $r$ term in Eq. 3.21 can also be made deliberately larger than 1. The linear dependence of the phase shift correction factor on the relative transmission ratio $r$ of the quarter wave 
plate implies an orientation preference between fast and slow axes not present when the ratio is unity. In other words, for $r \neq 1$, a phase shift gain would be a phase shift decrement if the quarter wave plate is rotated by $90^{\circ}$. In the far-infrared it can be assumed that $r$ will not be strictly unity for any quarter wave plate. This is due both to dichroismatic effects of any birefringent material and the etalon effect of different phasing between successive multiple reflections for the two principle axes. This latter effect does not apply to half-wave plates, where the optical path differences are always an integer number of wavelengths. The following optical constants of quartz are assumed $[72]$ for $184.6 \mu \mathrm{m}$ radiation: $n_{o}=2.117, \alpha_{o}=0.1 \mathrm{~cm}^{-1}$, and $n_{\mathrm{e}}=2.159, \alpha_{\mathrm{e}}=0.5 \mathrm{~cm}^{-1}$, where $n$ is the refractive index, $\alpha$ is the absorption constant, and the $o$ and $e$ subscripts denote the ordinary and extraordinary axes. For a $1.1 \mathrm{~mm}$ quartz plate, $r$ is found to be either 1.13 or 0.88 depending on the orientation of the plate. This in itself is large enough to indicate the need for a one-time calibration. Etalon effects are the dominant cause which produce an effective $r \neq 1$, and therefore antireflection coatings would eliminate this effect. Calculations show that $r$ can be enhanced to about 1.5 by tilting the waveplate at about $17^{\circ}$ to maximize the difference in reflection losses. This increase in $r$ is the result of increased transmission of the ordinary wave rather than a decrease in the extraordinary wave. In this case, there would be no amplitude decrement, as is the case when absorption dominates the value of $r$. For $r$-enhancement produced by an absorption differential, the amplitude decrement is given by the square of the enhancement factor.

The utility of phase shift enhancement aepends on the gain in ratio of phase signal to noise. The noise in phase is ultimately related to the signal to noise ratio on the carrier; therefore, amplitude decrements at some point must increase the noise on the phase measurement. For the last case considered, where etalon effects are used to increase $r$, there is clearly a gain since there is no amplitude decrement that results. Hence, there is a direct gain in phase signal to noise. There are two other cases when there would clearly be a gain. The first case is when the accuracy of the calculated phase shift is limited by effects other than noise on the carrier signal (e.g., finite digitization limits). The second is when phase detectibility is indeed limited by noise on the carrier but the amplitude decrement scales only linearly with the enhancement factor. In this case there is the customary square root advantage in signal to noise. These increased phase shifts do, however, come 
at the expense of having to make the required calibration measurement. For the case where the amplitude decrement scales as the square of the enhancement factor, there is no net gain in phase signal to noise.

Analysis of errors in polarimetry by use of Jones matrices has demonstrated the sensitivity of different schemes for measuring Faraday rotation in a plasma to various imperfections. Particular attention has been paia to the heterodyne phase shift method because it is insensitive to spurious amplitude variations. Less emphasis has been given to amplitude polarimetric techniques because the constrained access on ZT-40M prohibits the use of such methods. Consideration of real FIR optical components demonstrates that even with a phase shift measurement, if an accuracy of $10 \%$ is required, some form of a one-time calibration is necessary. The analysis also indicates that the possibility exists to exploit extreme adjustment errors to enhance the apparent phase shift by up to an order of magnitude. 


\section{Chapter 4}

\section{Description of System Employed for Single-Chord Measurements on $\mathrm{Z}$ T-10M}

Interferometric and polarimetric techniques have been developed for measurements of the density and poloidal field on ZT-40M. The index of refraction of the plasma detected by interferometry yiclds the integral $\int n_{e} d l$; Faraday rotation detected by polarimetry yields the integral $\int n_{e} \vec{B} \cdot \overrightarrow{d l}$. These measurements, made simultaneously on several chords, can be inverted to give density and poloidal field profile information. The details of the data unfolding, and the accuracy to which these profiles can be calculated a e presented in Chapter 2. The following sections describe the experimental apparatus used for single-chord measurements on ZT-40M.

Much of the assembly of the equipment was done before this thesis research was initiated by Bausman, et al., [49]. Without that effort, the final development and implementation on ZT-40M would not have been possible within the scope of a thesis. The system was composed of an FIR laser operating at $185 \mu \mathrm{m}$, a rotating grating for generating heterodyne signals, hollow dielectric waveguide beam propagation, and $\mathrm{GaAs}$ photo conductive detectors operating at $4.2^{\circ} \mathrm{K}$. Significant modifications of the initial equipment and techniques were required in order to obtain sufficient sensitivity for successful measurements on ZT-40M.

Although this system was sufficient for ceveloping and implementing singlechord measurements, inefficiencies and poor design of some of the components prevented multi-chord measurements. The design of a system that will support multi-chord measurements is presented in Chapter 7. Readers interested in the 
design of an interferometer/polarimeter are referred to Chapter 7 as well as the present section for discussions of the diagnostic design issues.

\subsection{Far-Infrared Laser as a Radiation Source}

The choice of radiation source wavelength is based on the wavelength dependence of the plasma phenomena of interest. Both the density-induced interferometric phase shift and the magnetic field-induced Faraday rotation increase with longer wavelengths. The more restrictive mininum criterion on the wavelength is given by the requirement that measurable Faraday rotation be induced by the plasma. The instrument developed for measurements on ZT-40M has a phase resolution of $\sim 5 \mathrm{mrad}$ at a response time of $25 \mu \mathrm{sec}$ (th) rerolution can be improved at a sacrifice of response time if required;. Therefore, a noxuinal rotation of $\sim 50 \mathrm{mrad}$ should be measurable with $10 \%$ accuracy. For the case of ZT-40M low current discharges, where typical parameters are: $n_{c} \sim 10^{19} \mathrm{~m}^{-3}, B_{\theta} \sim B_{\phi} \sim 0.1 \mathrm{~T}, L \sim 0.4 \mathrm{~m}$, and the wavelength required to induce $2 F \sim 50 \mathrm{mrad}$ is:

$$
\lambda=\frac{1}{2.63 \times 10^{-25}}\left[\frac{F}{n_{e} B_{\theta} L}\right]^{1 / 2} \simeq 500 \mu n
$$

For an upper limit on $\lambda$, it is important to consider the level of filiptization (Cotton-Mouton effect) generated for high current operation of ZT-40M, with $B_{\theta} \sim$ $B_{\phi} \sim 0.5 T$ and $n_{e} \sim 10^{20} \mathrm{~m}^{-3}$. The elliptization is given by Equation 3.8, which can be approximated:

$$
\beta \simeq 2.45 \times 10^{-29} \lambda^{3} n_{\mathrm{e}} B_{\phi}^{2} L \simeq 1.5 \mathrm{mrad} .
$$

This level of elliptization is negligible for amplitude polarimetry as well as heterodyne phase-shift polarimetry. (Recall from Section 3.3 that Signal $\sim 2 F-\beta^{2} / 4$ for small $F$ and $\beta$.j Fr measurements on tckamak plasmas, the elliptization is much more severe, because of the much stronger transverse magnetic nield $\left(B_{\phi} \sim 10 B_{\theta}\right)$. The eiliptization in this case could be as much as 150 mrad. Although orientation of the polarization at $0^{\circ}$ or $90^{\circ}$ to the transverse field eliminates the elliptization ef. fects, the Faraday rotation ( $\sim$ radian for the high current parameters) causes this orientation to change along the path of the beam. Therefore, the tokamak transverse field elliptization can cause serious errcrs in measuring the Faraday effect by 
amplitude polarimetry at $500 \mu \mathrm{m} .{ }^{1}$ The amount of elliptization ( $\lambda^{2}$ dependence) relative to the Faraday rotation ( $\lambda^{2}$ dependence) could be reduced by operating at a shorter waveleagth; however, the time response of the measurements would probably have to be sacrificed in order to resolve the smaller Faraday rotation.

Another maximal limit to the wavelength is governed by refractive effects, which are stronger at long wavelengths. The plasma acts as a negative cylindrical lens, and with the assumption of a parabolic density profile, Shmoys [73] calculates the maximum beam deflection to be

$$
\theta_{o}=\sin ^{-1}\left(\frac{\omega_{p}^{2}}{\omega^{2}}\right)
$$

which occurs at the major radial position

$$
R=R_{o} \pm a\left[\frac{1}{2}\left(1-\frac{\omega_{p}^{2}}{\omega^{2}}\right)\right]^{1 / 2},
$$

where $a$ is the plasma minoz radius and $R_{\mathrm{o}}$ is the major radius of the magnetic axis. The tolerance of refractive effects is machine dependent. For machines with constrained diagnostic access, even slight refraction can cause vignetting on apertu :cs, while machines with ample access might tolerate rather severe bending. For most plasmas of thermonuclear interest, radiation wavelengths that result in measurable Faraday rotation, yet are insensitive to Cotton-Mouton and refractive effects, are in the far-infrared region of the electromagnetic spectrum. Within the FIR region, the choice is governed by the availability of high-power laser lines.

The system developed for measurements on ZT-40M was actually designed for the nominal predicted ZT-40 plasma parameters at the time the diagnostic development was initiated, with $B_{\theta} \sim 0.5 T$ and $n_{\varepsilon} \sim 10^{21} \mathrm{~m}^{-3}$. Using the high-power laser line at $184.6 \mu \mathrm{m}, 1$ radian of Faraday rotation would be induced by this plasma. Since that time, the parameters of $\mathrm{ZT}-40 \mathrm{M}$ have changed drastically, to a regime of lower density, higher temperature, and dramatically improved stability. With $n_{e}$ in the $10^{19}-10^{20} \mathrm{~m}^{-3}$ range and $B_{\theta}$ in the $0.1-0.5 T$ range, only $10-100 \mathrm{mrad}$ of Faraday rotation is induced, and much of the effort in the diagnostic development has been directed towards improving the resolution of the polarimeter to allow the

\footnotetext{
${ }^{1}$ For heterodyne phase-shift polarimetry, the supression of elliptization results from aligning optical components relative to the transverse field, and does not degrade when the Faraday rotation becomes large.
} 


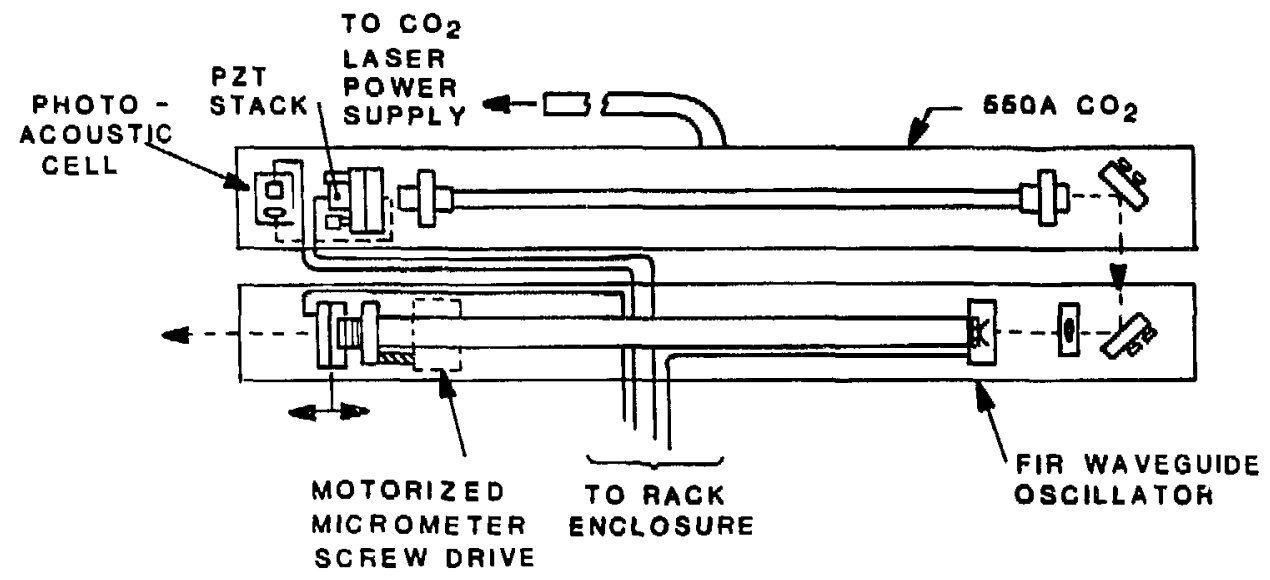

Figure 4.1: Schematic of FIR Laser

measurement of small rotation angles. Future operation at higher current levels will increase the rotation.

In retrospect, commitment to the short wavelength was not as unfortunate as it might appear. For ZT-40M, the diagnostic access is so constrained that vignetting by the entrance and exit apertures has been observed even at $184.6 \mu \mathrm{m}$. Although the level of beam attenuation that results has little effect on heterodyne phase-shift polarimetry, operation at longer wavelengths might have resulted in a serious loss of signal due to refractive effects.

The laser used for the single-chord measurements on ZT-40M was an Apollo model 118 Far-Infrared Waveguide Laser System. A schematic of the optical components appears in Figure 4.1. An Apollo model $550 \mathrm{~A}$ laser is used to optically pump the FIR laser. The physics of $\mathrm{CO}_{2}$ lasers is described by Pollack [74], and a useful review of $\mathrm{CO}_{2}$ laser design is presented by Hodges and Foote [75][76]. The $\mathrm{CO}_{2}$ laser is pumped with an electrical discharge, with a central anode operating at a voltage of $\sim 12 \mathrm{kV}$, and $\sim 40 \mathrm{~mA}$ of current flowing to the cathodes (fioating at $\sim 2 \mathrm{kV}$ ) at each end of the discharge tube. The $Z n S e$ output coupler and Brewster window form the vacuum seals for the discharge. The gas used for the $\mathrm{CO}_{2}$ 
laser is a commercially available $\mathrm{mix}^{2}$ of $75.2 \% \mathrm{He}, 18.4 \% \mathrm{~N}_{2}$, and $6.4 \% \mathrm{CO}_{2}$. The nitrogen molecules are collisionally excited in the discharge and transfer energy to the $\mathrm{CO}_{2}$ molecules, selectively exciting the $\mathrm{CO}_{2}$ molecules to the desired energy state. After the $\mathrm{CO}_{2}$ molecule emits radiation, the helium serves to collisionally extract the residual energy to return the $\mathrm{CO}_{2}$ molecule to the ground state. The gas is flowed through the system at a pressure of $\sim 10$ Torr, and it is found in practice that more power is obtained by flowing the gas faster. The maximum power that has been obtained with no tuning element in the laser is $\sim 50$ watts, with most of the power in the $10.6 \mu \mathrm{m}(10 \mathrm{P22})$ line of the $\mathrm{CO}_{2}$ spectrum. The $\mathrm{CO}_{2}$ line required to pump the FIR laser gas is the $9.2 \mu \mathrm{m}(9 \mathrm{R32})$ line, which is a less efficient line for the $\mathrm{CO}_{2}$ laser, where a maximum power of $\sim 30$ watts has been obtained. The wavelength selection is performed by grating a that forms the back reflector of the $\mathrm{CO}_{2}$ cavity. The grating was designed for $9.6 \mu \mathrm{m}$ radiation, at a blaze angle of $30^{\circ}$, and is tilted slightly to reflect $9.2 \mu \mathrm{m}$ radiation in the first order. The output coupler is a 10 meter focal length, $75 \%$ reflector, which forms a half-symmetric cavity with the grating back-reflector. The $\mathrm{CO}_{2}$ beam is sent through a $Z n S e$ inverted telescope and injected into the FIR laser through a $2 \mathrm{~mm}$ hole in the back-reflector of the FIR cavity.

The angular alignment controls of the $\mathrm{CO}_{2}$ laser are adjusted manually; however, feedback stabilization of the cavity length is required for reliable operation. The $\mathrm{CO}_{2}$ cavity length is controlled by mounting the grating on a piezo-electric translator (PZT) mount. A small periodic signal is applied to the PZT to modulate the length of the $\mathrm{CO}_{2}$ cavity, which causes a modulation of the $\mathrm{CO}_{2}$ wavelength. The zeroth order reflected from the grating, which would otherwise be lost, is directed to a gas cell containing the FIR laser gas at a pressure of $1-3$ Torr. The modulating $\mathrm{CO}_{2}$ beam produres a modulation in the power absorbed in the cell, which results in the generation of acoustic vibrations in the gas (known as the opto-acoustic effect[77]). These vibrations are sensed by a common microphone. A phase comparitor monitors the relative phase of the disturbance signal and the microphone signal. The amplitude, phase, and frequency of the microphone signal depend on the relation between the $\mathrm{CO}_{2}$ wavelength and the FIR gas absorption curve (which, for this purpose, can be thought of as the gain curve for the FIR laser). At the optimal $\mathrm{CO}_{2}$ cavity length the microphone signal is twice the fre-

\footnotetext{
2e.g., Matheson Gas Products, Inc.
} 


\begin{tabular}{|c|c|c|c|c|c|}
\hline$\lambda(\mu m)$ & gas & pump & power (mW) & & \\
\hline 117.7 & $\overline{\mathrm{DFM}}$ & 9R20 & 100 & & \\
\hline 118.8 & $\mathrm{MA}$ & 9P36 & 150 & & \\
\hline 152.9 & NH3 & 10R18 & 200 & & \\
\hline 184.6 & DFM & 9R32 & 250 & $\begin{array}{r}\text { DFM } \\
\text { MA }\end{array}$ & $\begin{array}{l}\text { - Difluoromethane } \\
\text { - Methyl Alcohol }\end{array}$ \\
\hline 189.9 & DCN & Discharge & 200 & NH3 & - Ammonia \\
\hline 194.7 & DCN & Discharge & 200 & $\begin{array}{l}\text { DCN } \\
\text { HCN }\end{array}$ & $\begin{array}{l}\text { - Deuterium Cyanide } \\
\text { - Hydrogen Cyanide }\end{array}$ \\
\hline 214.6 & DFM & 9R34 & 200 & MBR & - Methyl Bromide \\
\hline 336.6 & $\mathrm{HCN}$ & Discharge & 200 & MI & - Methyl Iodide \\
\hline 415.0 & MBR & 10R02 & 20 & & \\
\hline 447.1 & MJ. & $10 \mathrm{P} 18$ & 20 & & \\
\hline 660.7 & MBR & 10R20 & 20 & & \\
\hline
\end{tabular}

Table 4.1: Laser Gases

quency of the PZT disturbance signal, and therefore the optimal cavity length is maintained by maximizing this doubled frequency. A.t other cavity lengths, the micruphone signai has the same frequency as the disturbance signal, and the phase of the microphone signal depends on which side of the FIR absorption curve peak the $\mathrm{CO}_{2}$ laser is operating. If the $\mathrm{CO}_{2}$ cavity is off of optimum, a DC error voltage is applied to the PZT in the direction indicated by the relative phase of the disturbance and microphone signals until the doubled frequency is maximized.

The FIR laser has a 1.5 meter long $38 \mathrm{~mm}$ diameter waveguide cavity. The back-reflector and output coupler are gold-coated pyrex plano mirrors. The backreflector has a $2 \mathrm{~mm}$ diameter hole for $\mathrm{CO}_{2}$ injection and the output coupler has a $10 \mathrm{~mm}$ diameter hole, commonly known as a "hole coupler." A crystalline quartz vacuum window, coated for $\mathrm{CO}_{2}$ reflection, is mounted beyond the hole coupler.

The wavelength used for measurements on $\mathrm{ZT}-40 \mathrm{M}$ is generated by the $184.6 \mu \mathrm{m}$ transition in difluoromethane $\left(\mathrm{CH}_{2} \mathrm{~F}_{2}\right.$ or DFM), which was pumped by the $9 R 32$ line of the $\mathrm{CO}_{2}$ laser[78]. This particular wavelength was chosen because of the high power that could be achieved from this transition in DFM, along with the Faraday rotation and refraction criteria discussed earlier. Table 4.1[79][78] shows the various laser gases and wavelengths that could be used for polarimetry. DFM 
had the advantage of being able to produce power for several days with a single static gas fill, eliminating the need for a flowing system. The gas also freezes at liquid nitrogen temperatures. With a simple cold-trap gas recovery system, the fill gas can be recycled and purified, which is done daily as a routine. A flowing gas system, where the used gas is vented would be prohibitively expensive. However, the liquid nitrogen cold-trap could be used as a pump for the flowing gas system, and the gas could be recovered. No tests were performed to determine if more power could be obtained by flowing the gas in this manner.

The output of the FIR laser is linearly polarized in a direction perpendicular to the $\mathrm{CO}_{2}$ pump beam polarization. The $\mathrm{CO}_{2}$ laser is vertically polarized (determined by the orientation of the grating), therefore the FIR polarization is horizontal.

\subsection{Possible Heterodyne Techniques}

The use of heterodyne detection for interferometry and polarimetry was discussed in Chapter 3. This involves the use of two different frequencies which produce beats when combined. Heterodyne techniques offer the following advantages over single-frequency methods:

- The beat frequency can be freely chosen. The frequency can be tailored to the specific time response requirements for the measurement of interest.

- Use of heterodyne techniques can result in enhanced signal to noise ratios, because all but the heterodyne beat frequency can be discriminated against.

- Heterodyne techniques cause the physical effects of interest to modify the time signature of the beat signal. The measurements are insensitive to effects that cause spurious changes in the signal amplitude.

At visible and near infrared wavelengths, acousto-optic methods are frequently employed to generate a frequency shift on a portion of a laser beam. A singlefrequency laser beam is sent through an acousto-optic modulator, where part of the beam is shifted in frequency by traveling acoustic waves. The waves act as a moving diffraction grating which Doppler-shifts the frequency of the laser beam component that interacts with the wave. Although acousto-optic effects have been 
observed at far-infrared wavelengths[80], there is no efficient acousto-optic wiowistor that has been developed at such wavelengths. There are a number of alternative techniques that can be employed. The state of the radiation frequency and polarization that is required for heterodyne phase-shift polarimetry and interferometry is opposite circular polarizations at a slight frequency difference (see Fig. 3.6). This is equivalent to a linear polarization rotating at half the difference frequency. The techniques that could be used to generate this condition are listed in order of preference, although the best method is still developmental:

1. Electro-optic rotation of the $\mathrm{CO}_{2}$ pump beam polarization, cansing the FIK polarization to also rotate,

2. Combining two FIR lasers operating at slightly different frequencies, and

3. Mechanically doppler-shifting a component of a single beam.

These techniques will be described more fully below.

\subsubsection{Electro-optic Rotation of the Pump Polarization}

Electro-optic techniques have been developed for the infrared wavelengths. Rotation of the $\mathrm{CO}_{2}$ pump polarization should be possible [81]. As long as there are no polarizing alements in the FIR cavity, the FIR polarization should track the pump polarization. The concept is illustrated in Figure 4.2. The rotating linear polarization is equivalent to counter-rotating circular polarizations at a frequency difference, which is the required polarization state for heterodyned polarimetry. This technique has important advantages over the other opticns in ease of alignment and stability of the frequency difference. The technique uses simple transmission optics, and does not require a precise overlapping of initially spatially separated frequency offset beams, as in other techniques. The difference frequency is controlled by a signal generator driving the electro-optic crystal, which would give clear and stable frequencies. The driving signal could also be conveniently used as a reference signal for the phase shifts induced by the plasma, eliminating the need to use a detector to moritor an input difference frequency that is not as stable.

The present drawback of the electro-optic modulatios. technique is the unavailability of commercial components and the uncertainty of performance capabilities 

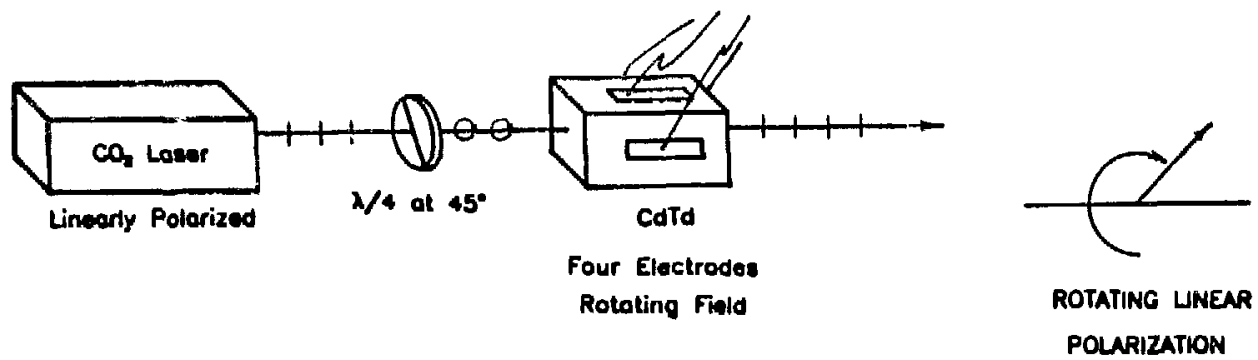

Figure 4.2: Heterodyning by Rotating Pump Polarization

in terms of possible frequency limits. The frequency could be limited by restrictions in how fast the polarization can be rotated by electro-optic crystal and drivers. The frequency could also be limited by a "time constant" associated with the FIR laser polarization tracking the pump polarization. Mansfield and Johnson [82] performed experiments on a similar concept, where the pump polarization was rocked through small angles. This is an alternative to using a ferrite modulator on the FIR beam for the amplitude polarimetry scheme (see Fig. 3.5) at shorter wavelength, where the ferrite modulator is very inefficient. They were able to rock the pump polarization through angles of $0.5^{\circ}$ at a frequency of $\sim 10 \mathrm{kHz}$. It is unknown as to whether larger angles can be obtained. These fundamental issues need to be investigated before the utility of electro-optic modulation for heterodyne signal generation can be assessed.

\subsubsection{Twin FIR Lasers}

Twin FIR lasers have been used for heterodyne interferometry for several years [83]; however, their use has been limized because of the difficulty of maintaining a stable frequency difference between the radiation from the two lasers. Recent refinements in design and stabilization techniques have improved the performance of two-laser systems, and this technique for generating heterodyne signals is probably 


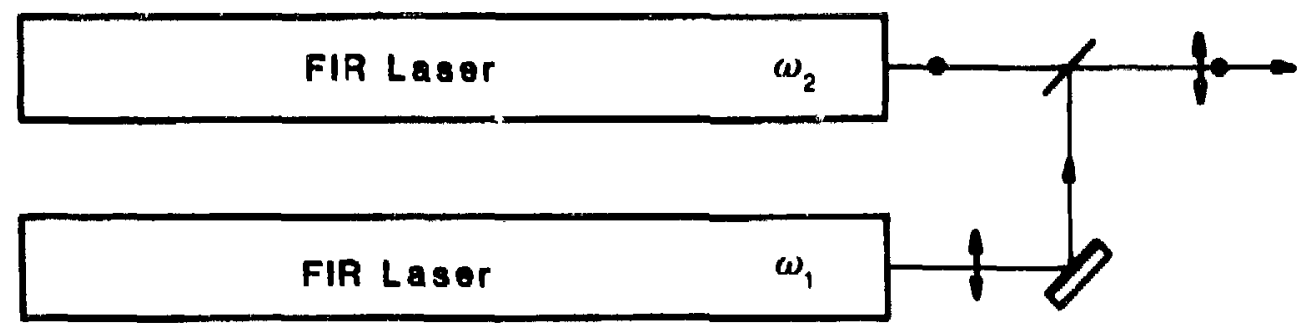

Figure 4.3: Heterodyning by Using Frequency-Offset Lasers

the method of choice at this time.

The idea behind the two-laser technique is to operate two separate lasers at a slight frequency difference, and combine the outputs with their polarizations orthogonal. This concept is illustrated in Figure 4.3. By sending the combined beams through a quarter-wave plate (see Appendix C), frequency-shifted circular polarizations are obtained. The difference in frequency is achieved by aligning the lasers with slightly different cavity lengths, which forces the lasers to operate at different wavelengthe within the gain curve of the lasing medium. The maximum difference frequency that can be achieved depends on the width of the gain curve. Difference frequencies of $1-5 \mathrm{MHz}$ are typically obtained with this method. Using piezo-electric mounts on the FIR cavities and feed-back stabilizing the lasers for maximum output power at a specified difference frequency could make the system quite reliable. Peebles, et al. [84], have demonstrated a simplified system where the two FIR lasers share a common back-reflector and output coupler. These optical elements are large enough to accept side-by-side waveguide tubes, and a step can be put on one of the reflectors, precisely determining the frequency difference. In practice it was found that a reflector without a step could be used, and the cavity length difference achieved by slightly tilting the common reflector. The angular misalignment required was not enough to cause noticeable degradation of laser power. Although a two-laser system has the advantage of providing double 


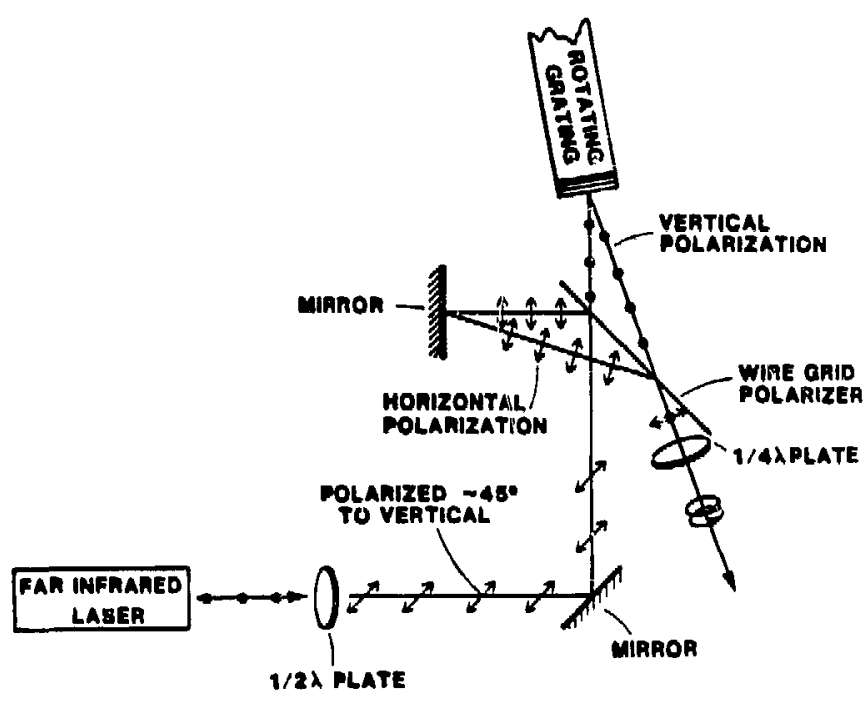

Figure 4.4: Heterodyning by Using Rotating Grating

the laser power of an electro-optic modulated system, it does require a dedicated detector to monitor the difference frequency changes.

\subsubsection{Rotating Grating}

The development of rotating gratings for mechanical heterodyning took place several years ago. Some of the contributors include Veron[85] and Peterson[86]. A component of the beam is reflected off the rotating cylindrical grating (see Figure 4.4). Grooves that form the grating pattern are machined on the circumference of an aluminum disk (somewhat like grooves on the edge of a coin). The shape of the grooves is designed such that the resulting grating is blazed so the laser beam is efficiently reflected off the rotating wheel. The rotation of the wheel causes the grating pattern to move, making this the mechanical analogue of the acousto-optic modulation techniques discussed earlier.

The dimensions of the grating pattern are determined by the following equation:

$$
\frac{m \lambda}{\cos \theta}=d(\sin \alpha+\sin \beta)
$$

where $m$ is the order of the grating, $\alpha$ and $\beta$ are the usual angles of incidence and diffraction in the vertical plane (see Figure 4.5) given in most textbooks (e.g., $[87][88]$ ). The angle $\theta$ is the azgle of incidence and reflection in the horizontal 

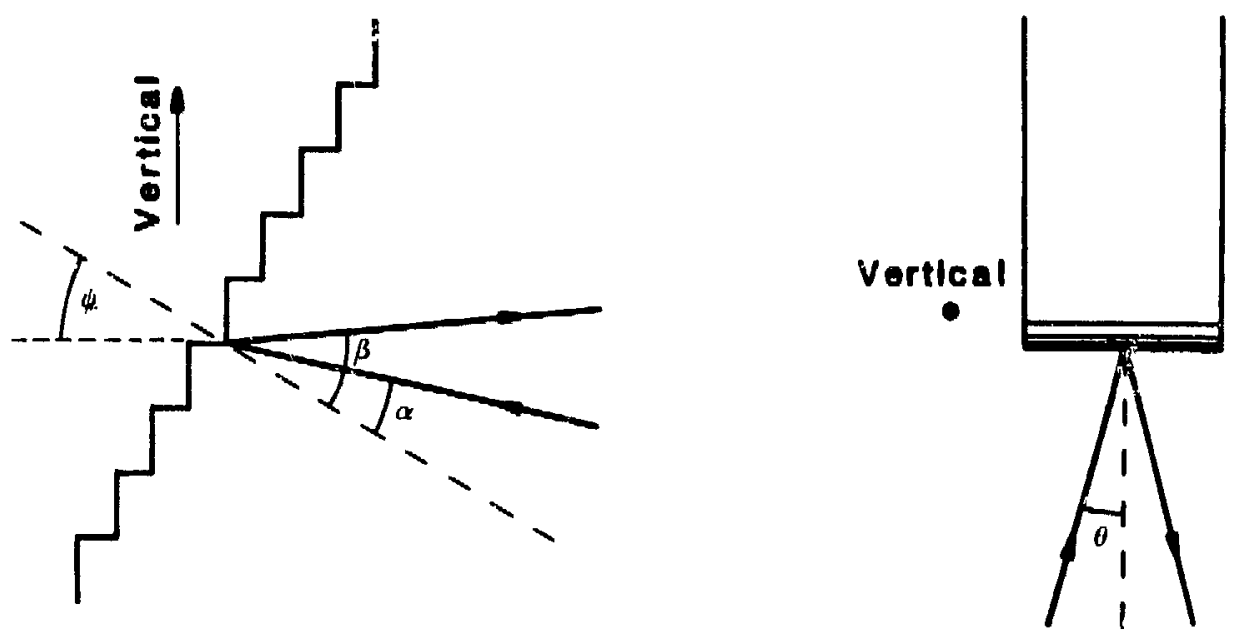

Figure 4.5: Grating Geometry

plane, which is not usually included in the grating equation, but is important in this application. The Doppler frequency shift that is generated by the grating is given by:

$$
\Delta f=\frac{2 f}{c} R \omega \cos \theta \sin \alpha,
$$

where $f$ is the radiation frequency, $R$ is the radius of the wheel, $\omega$ is the rotational frequency of the wheel, $\psi$ is the angle between the grating normal and horizontal directions, and $c$ is the speed of light. The frequency shift can be increased by making $\psi$ larger, however, cylindrical effects become severe if the beam is reflected too far below the midplane. Cylindrical effects can be reduced by increasing the radius of the wheel and focusing the laser beam down to a small area of the grating.

Although the rotating grating has not suffered from severe stability problems as did the early two-laser systems, it is a poor optical element. Since it is cylindrical, it causes the reflected beam to diverge more strongly than the beam reflected from the mirror. In addition, unless sare is taken to isolate the vibrations induced by the rotation, they can be sever ? enough to affect the laser performance. Even if they are isolated, these vibrations generate spurious phase changes on the heterodyne signal, and a dedicated detector is required to monitor the vibrational phase shifts in order to be able to extract them from measurements of the plasma. Also, grating ghosts (imperfections in the grating fabrication), and drifts in the rotational velocity of 


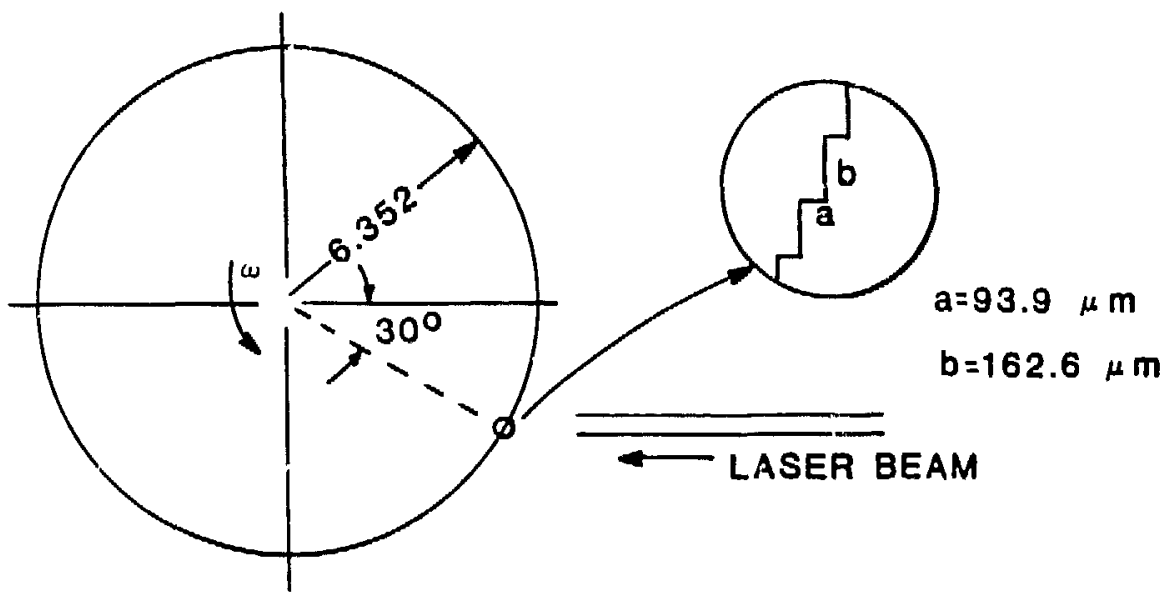

Figure 4.6: Grating used for Measurements on ZT-40M

the wheel create anomalies that also need to be extracted by examining the signal from the monitor or reference detector. These effects will be discussed in much more detail in the following section. The grating has further disadvantages in efficiency ( $\sim 50 \%$ overall) and complicated alignment, both worsened by machining imperfections.

\subsubsection{Rotating Grating used for Measurements on ZT-40M}

After the discussion of the preceding section, it may be surprising to learn that the heterodyne technique employed for measurements on ZT-40M used a rotating grating. The original decision to use a rotating grating was based on the poor stability of the two-laser systems at the time. After that, there was little incentive to retro-fit a two-laser system, because the grating was not limiting the performance of the overall diagnostic system. The next generation system will probably use two lasers to produce the heterodyne signals.

The grating used for measurement on ZT-40 is shown in Figure 4.6. The grating is made from a $\sim 13$ " diameter, 1 " thick aluminum disk, with the grating ruled on the edge of the disk. The grating pattern is designed to reflect $184.6 \mu \mathrm{m}$ radiation in first order $(m=1)$ at $\alpha=\beta=\psi=30^{\circ}$ below the midplane, and have a lorizontal angle of incidence $\theta=10^{\circ}$. It was found that the grating could also be 
used at the $214.7 \mu \mathrm{m}$ line of DFM if the orientation of the grating was changed such that $\alpha=\beta=\psi=35^{\circ}$ and $\theta=15^{\circ}$. Therefore, there is a small amount of flexibility in the application of the grating.

The grating was ruled in four discrete segments, each covering $60^{\circ}$ of the circumference. It was not ruled completely around its circumference to save machining costs. This would have been adequate for measurements of initial parameters of ZT-40 (see page 95 in Section 4.1); however, the segmented grating imposed a limit on the heterodyne frequency for standard $\mathrm{ZT}-40 \mathrm{M}$ parameters. At faster rotational

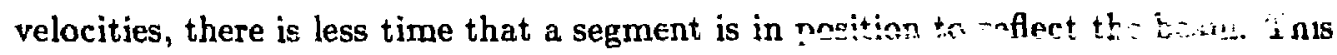
time for $a 60^{\circ}$ segment is given by:

$$
t=\frac{\pi}{6} \frac{\Delta f}{f} \frac{c}{R \cos \theta \sin \psi}
$$

In order to make measurements during the entire $2 T-40 M$ pulse length of $\sim$ $20 \mathrm{msec}$, the heterodyne frequency is limited to $\Delta f \leq 40 \mathrm{kHz}$.

A schematic of the Dopples shifting configuration appears in Figure 4.7. The linearly polarized output of the FIR laser is sent through a $\lambda / 2$ plate. The axis of the $\lambda / 2$ plate is oriented such that the two orthogonal beam components are balanced. If all the optical elements were ideal, the beams would be balanced if the polarization beyond the $\lambda / 2$ plate was $45^{\circ}$ to the vertical. Since the grating is not ideal, a larger vertical component is required to compensate for the reflection losses of the grating. A wire grid polarizer directs the horizontal beam component to a stationary mirror, and transmits the vertical component to the rotating grating. Since the polarizer is rather poor in reflection (see Appendix C), a clean-up polarizer (not shown) is required just in front of the stationary mirror to more completely polarize the non-Doppler shifted component. The grating frequencyshifted the vertical component and the beams were recombined at the wire grid polarizer. A quarter wave plate then converts the orthogonal linear polarizations to orthogonal (or opposite) circular polarizations, which is the required polarization condition for the measurements.

The two beams are then injected into the waveguide used for propagation to ZT-4OM. The configuration of the grating and waveguide is such that radiation from DFM lines other than the 184.6 $\mu \mathrm{m}$ line can not enter the waveguide. This is particularly important for the $214.7 \mu m$ radiation, which is pumped by the $9 R 34$ $\mathrm{CO}_{2}$ transition (adjacent to the $9 \mathrm{R32}$ transition that pumps the $184.6 \mu \mathrm{m}$ line). 


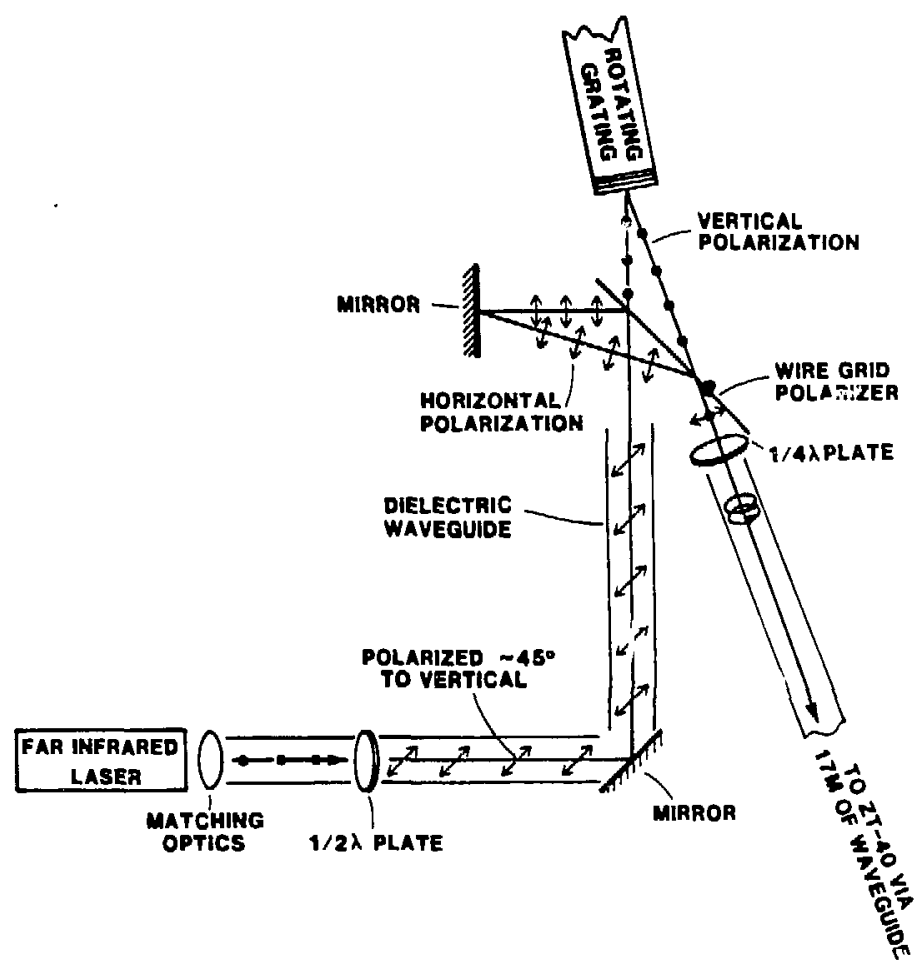

Figure 4.7: Doppler Shifting Configuration 
There are instances when the $\mathrm{CO}_{2}$ laser runs on both the $9 R 32$ and $9 R 34$ lines simultaneously, generating both $184.6 \mu \mathrm{m}$ and $214.7 \mu \mathrm{m}$ radiation. The grating also reflects the $214.7 \mu \mathrm{m}$ radiation, but the reflection angle is deviated enough to miss the waveguide. In this case, the wavelength selectivity of the grating is an advantage, filtering out unwanted radiation that might cause problems in other heterodyne schemes.

Several problems werc found with the rotating grating. The problems with the grating that most clearly hindered the performance of the system were grating ghosts, mechanical vibrations, and frequency drifts.

Very early in the development of this diagnostic, grating ghosts ware recognized as a severe problem. They produce a periodic phase and amplitude distortion on the heterodyne signal, a signature created by imperfections in the machining of the cylindrical grating. For this reason, the phase reference for interferometry or polarimetry can not be taken from a pure $40 \mathrm{kHz}$ signal, but needs to be taken from a signal produced by the same grating segment, which reproduces the same signature due to imperfections, and thereby cancels the phase distortions. This was conveniently done by recording the signal from the grating precisely one wheel revolution before the plasma discharge in addition to the signal during the discharge. The phases of the two signals were analyzed, and the difference between the phases, or the residual phase, should have contained the information about the plasma.

However, there was a problem with this scheme. Mechanical vibrations of the rotating grating, induced by the firing of the machine, generated phase information that was not seen in the earlier reference signal. The vibrational structure could not be separated from the plasma information. Figure 4.8 shows the analyzed phase of a typical reference signal. The fast frequency oscillation $(1.4 \mathrm{kHz})$, due to grating ghosts, was a repeatable signature of the grating, while the slow frequency component $(120 \mathrm{~Hz})$ was due to vibrations, and is different from the vibration structure measured during the plasma discharge.

A similar problem can result from drifts in frequency due to slow changes in the rotational velocity of the wheel. If the modulation frequency is slightly different in the plasma and reference signals, the result is a linear slope of the baseline of the residual phase trace. Such a phenomenon was observed even in the interferometric data when ZT-40 was operating at low current. Figure 4.9 shows the density 


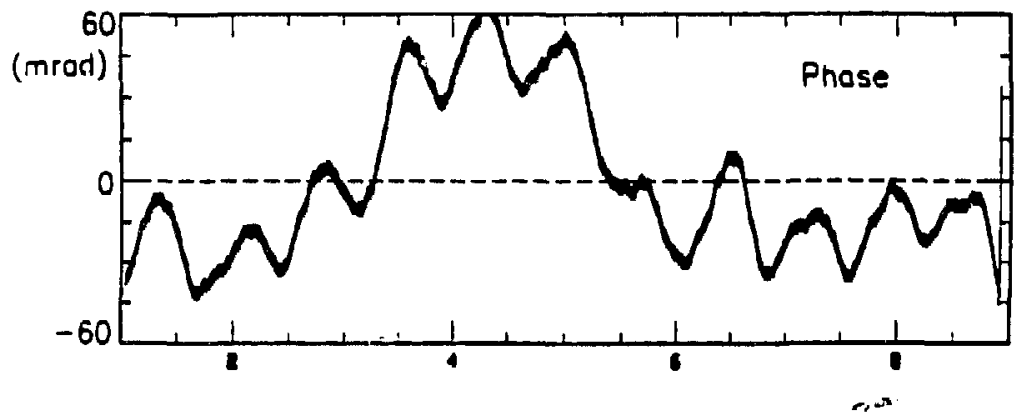

Figure 4.8: Phase of a Typical Reference Signal

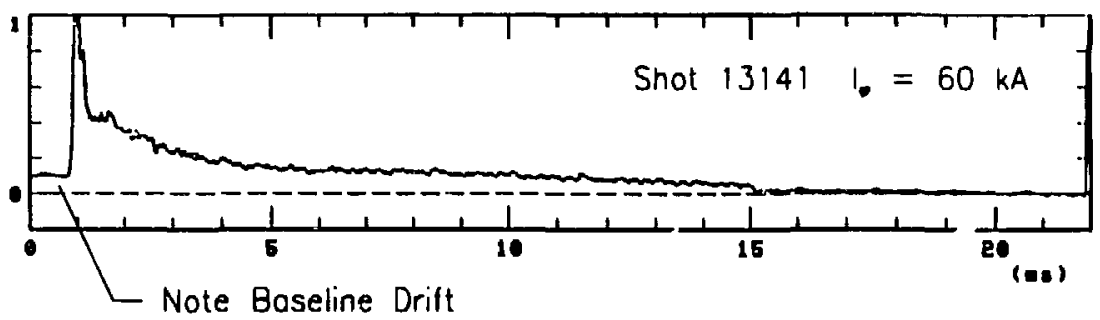

Figure 4.9: Density Information Superimposed on Baseline Drift

information superimposed on a baseline drift (the zero phase was defined at the end of the discharge). A frequency difference of one part in $10^{4}$ between successive wheel revolutions would account for this drift.

The obvious solution to the mechanical vibration and frequency drift problem was to incorporate a second detector to measure the reference signal simultaneously with the plasma signal. Then any phase distortions due to vibrations or frequency drifts would be present on both signals, and would not appear on the residual phase. The reference detector was installed in the laser screenroum, directing part of the beam to the detector with a mylar beam splitter before the 17 meters of waveguide. As a result of the installation of the second detector, the baseline drift was indeed eliminated.

There was, however, a different problem with this scheme. Although the vibrations and frequency drifts were successfully eliminated with the second detector, the grating ghosts were no longer canceled. The reason for this surprising result 
was that the phase distortions caused by the grating ghosts manifested themseives differently on the different detectors. This is illustrated in Figure 4.10. Figure 4.10a shows the signal from the reference detector as stored in the digitizer. The envelope amplitude modulations are real, not plotting artifacts, and are due to grating ghosts. Figure 4.10b shows the derived phase of the reference detector signal. Again, the $120 \mathrm{~Hz}$ oscillation was caused by vibrations, and the $1.4 \mathrm{kHz}$ oscillation was caused by grating ghosts. Figure 4.10c shows the signal from the plasma detector (taken with no plasma), and Figure 4.10d shows the derived phase of the plasma detector signal. Figure 4.10e shows the difference between the traces of Figures $4.10 \mathrm{~b}$ and $4.10 \mathrm{~d}$, or the residual phase. Since there was no plasma, this should be a flat baseline. As expected, the vibration structure was canceled, but, as can be seen by inspection, the amplitude of the ghost structure was higher on the reference signal phase, and the grating ghost features were not completely canceled.

The precise reason for the different phase errors on the two detectors is not clear. The amplitude of the envelope modulations of Figures 4.10a and 4.10c are different, and amplitude modulations will couple to phase modulations (the exact amount depending on the algorithm for deriving the phase - see Eq. 3.23). However, the signal with the smailer amplitude modulations was the signal with the larger phase modulations, exactly opposite from the expected result of amplitude modulation to phase modulation coupling. Even though inspection of the data does not seem to reveal the cause of the different phase errors on the different detectors, the difference in the location of the two detectors may have been the cause of the problem. The plasma detector measured signals that has passed through 17 meters of waveguide. Since the waveguide is a mode-selective device, it may have filtered out components of the beam that were not filtered for the reference detector. Hence the grating ghosts may have been partially filtered by propagating through the waveguide. A test of this would have been to move the reference detector beyond the 17 meters of waveguide, so both detectors would have the same filtering. Even in this case, however, the plasma signal would pass through the torus, which may further filter the beam berause of waveguiding in the diagnostir, tubulations.

Rather than determining the cause of the problem, steps were taken to circumvent it. Although they were brute-force in nature, these steps effectively eliminated the grating ghost errors while retaining the correction for vibrations and frequency drifts. In addition to the two-detector system, separate reference traces 
Grating Chosts and Mechanical Vibrations
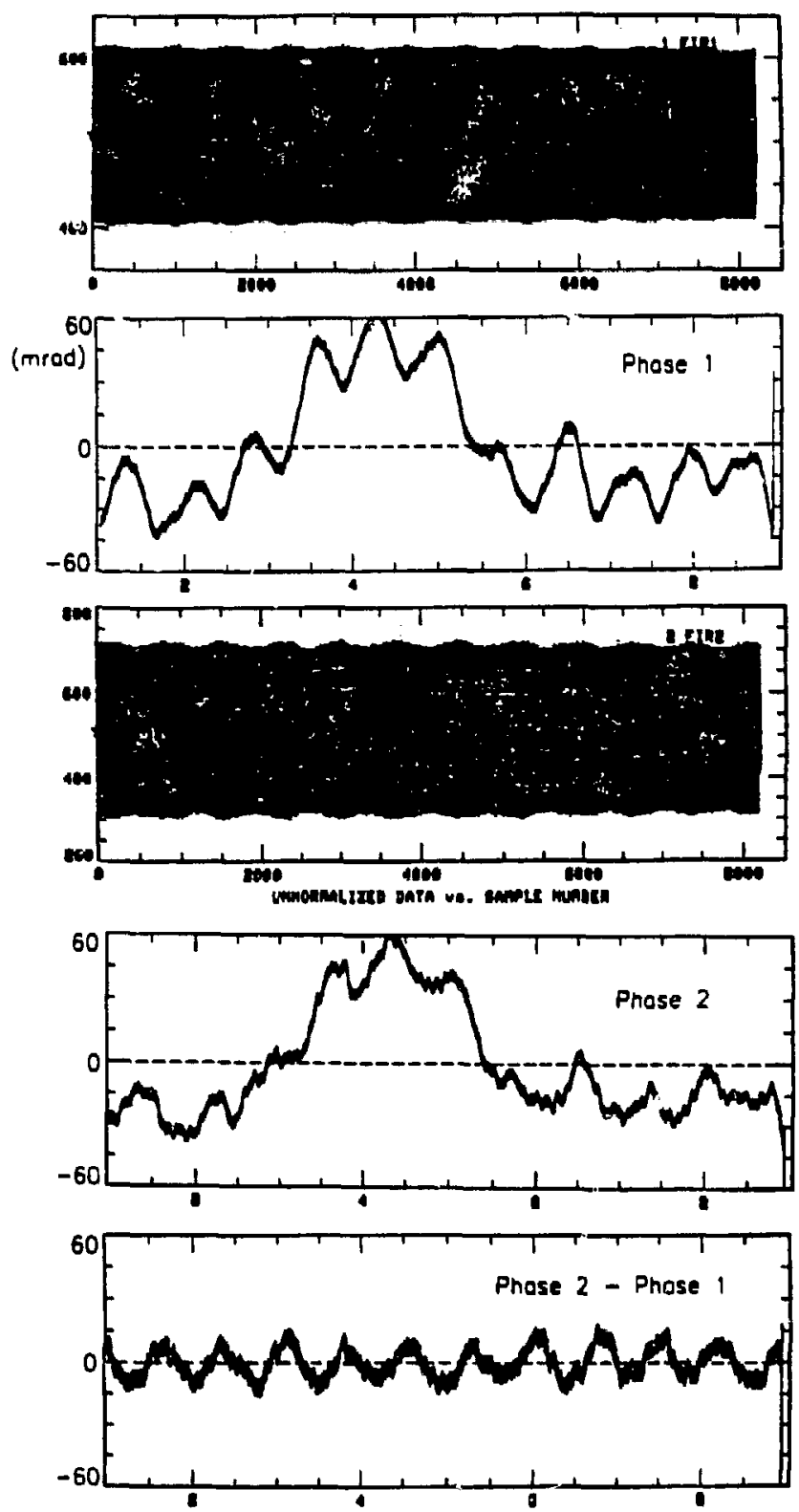

Figure 4.10: Grating Ghosts and Mechanical Vibrations 


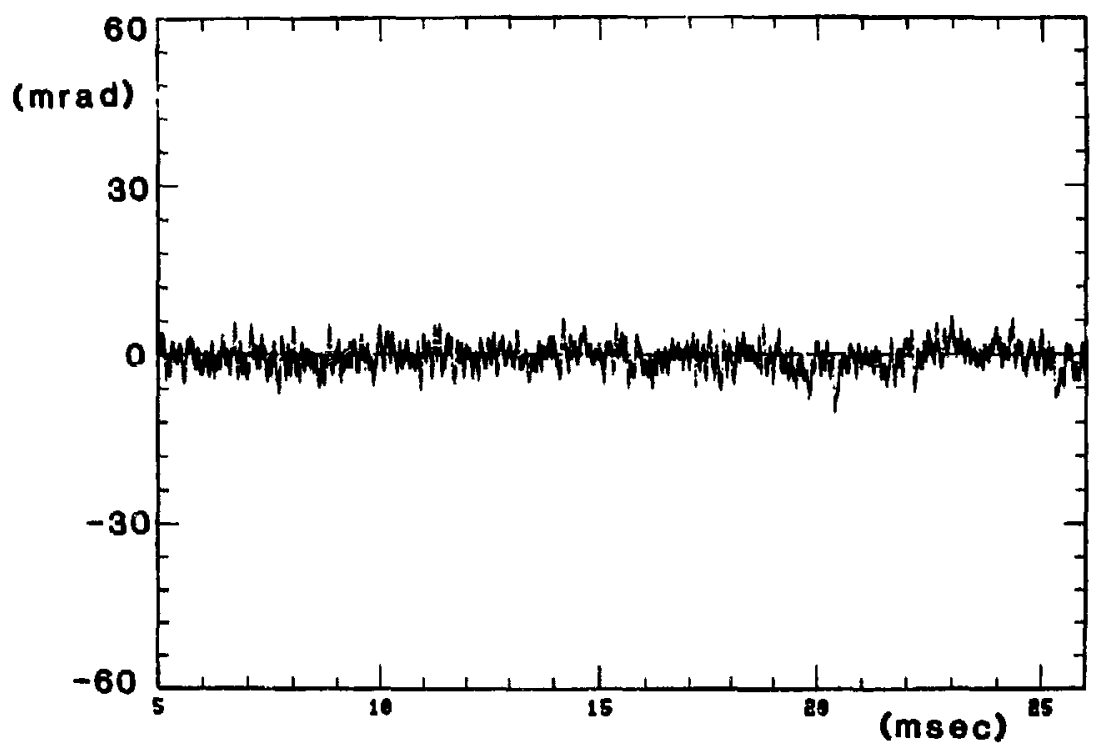

Figure 4.11: Baseline with Four-Signal Technique

were taken one wheel revolution before the plasma discharge on both detectors. This four-signal technique employed ihe two detectors to eliminate the vibrations and frequency drifts, and recorded the reference signals from the same grating segment to eliminate the phase errors due to grating ghosts. The resultant baseline is shown in Figure 4.11, indicating that the grating problems no longer affected the baseline. The residual noise was less than $3 \mathrm{mrad}$ ( $\mathrm{rms}$ ), and with the $25 \mu \mathrm{sec}$ time response, the "instrumental" sensitivity bandwidth product was substantially better than what has been achieved elsewhere $[43\},\{40]$.

\subsection{Waveguide Beam Propagation}

A waveguide was used to propagate the beam from the laser and rotating grating to ZT-40M. Useful descriptions of waveguide theory are presented by Marcatili and Schmeltzer [89] and Crenn [90] [91]. This is an alternative technique to using standard reflection and transmission optics (frequently referred to as an "optical train"): Use of waveguides for beam propagation in the FIR has the following advantages: 
1. Due to gaussian beam divergence of this relatively long wavelength radiation, an optical train requires large aperture optics. The beam has to be focused to counteract the divergence with lossy focusing lenses, or with focusing mirrors that need to be compensated for spherical abberations. Waveguide beam propagation uses smaller diameter turning mirrors and does not require focusing optics because the beam cannot diverge in the waveguide.

2. Many FIR radiation wavelengths are absorbed by moisture in the air, so the atmosphere in the beam path needs to ke dehumidified, typically by slightly pressurizing a beam enclosure with dry nitrogen. With waveguide propagation, the atmosphere can be conveniently enclosed and dehumidified, while an optical train requires a large and bulky beam enclosure.

3. A waveguide acts as a mode-filtering element, and can purify the mode of the beam, thus allowing optimal focusing of the beam through the vacuum vessel of $\mathrm{ZT}-40 \mathrm{M}$ without having to resort to spatial filtering techniques.

The type of waveguide frequently employed for FIR beam propagation is overmoded hollow dielectric waveguide. The dominant mode in this type of waveguide is the $E H_{11}$ hybrid mode, which is characterized by a linearly polarized electric field with a zeroth order Bessel function spatial profile. Upon exiting the waveguide, the profile quickly develops the tails of a Gaussian distribution. Therefore, no mode conversion is required for efficient injection into the waveguide, the exit beam can be efficiently focused, and the beam propagation beyond the waveguide can be accurately calculated with Gaussian beam propagation theory.

The waveguide used on the ZT-40M system consists of 1/2" I.D. glass tubing and $i$ " I.D. Lexan ${ }^{\infty}$ plastic tubing. The $1 / 2$ " tubing was used to propagate the beam from the laser to the grating, and to propagate the frequency-shifted beams for $\sim 1$ meter before converting to the 1 " waveguide. A mylar pellicle is mounted at $45^{\circ}$ at the entrance to the 1 " waveguide. The reflected radiation from the mylar is directed to the reference detector that monitors the beat signal generated by the grating, which allows corrections of the mechanical vibrations, frequency drifts, and grating ghosts described earlier. The mylar also seals the entrance to the waveguide, and dry nitrogen is injected at that point and flowed out the other end of the waveguide, which was left open. The dry nitrogen reduces the water-vapor absorption at $184.6 \mu \mathrm{m}$, which otherwise could cause a factor of 50 attenuation of 
the beam propagating through the waveguide. The system requires 17 meters of pathlength, and a total of $7-90^{\circ}$ bends to propagate the FIR beam from the laser to the machine. Mechanical techniques are used for the $90^{\circ}$ bends, where a fixture holds the entering and exiting waveguide sections at a $90^{\circ}$ relative angle, and the beam is reflected by a mirror that is adjustable so the transmission through the bend can be maximized.

The waveguide described above is the component of the system that has the most serious imperfections, resulting in many inefficiencies and inconveniences. These imperfections include high attenuation of the laser power, poor mechanical stability of the alignment of the waveguide, poor mode quality at the exit of the waveguide, and elliptization of linear polarization as it propagates through the waveguide (caused by significant sagging of the waveguide). The high atteruation is caused by the number of $90^{\circ}$ bends, the use of small diameter non-precision bore plastic tubing, and poor positioning and crude adjustment controls for the waveguide. This poor transmission $(T \sim 5-10 \%)$ dic not limit the ferformance of the single-chord measurements, but multichord operation requires more laser power at the machine. The instability of the waveguide was caused by inappropriate placement of the waveguide in a vulnerable location where it is frequently bumped out of alignment. This is only an inconvenience which requires more frequent realignment thar: $r$ ne would like. The elliptization of linear polarization was discovered when the extinction ratio of the interferometer was measured. This was found to be a result of a depolarizion in the waveguide when the polarizarion of the beam injected into the waveguide was mesasured to be $\sim 30: 1$ (which is the limit of the ability to measure this parameter), and the exit polarization was only $\sim 6: 1$. The introduction of a quarter waveplate restored the polarization to linear $(\sim 25: 1)$, indicating that it was not a depolarization of the linerr polarization, but an elliptization. As was demonstrated the elliptization can be corrested, but requires additional retardation plates which are expensive and somewhat attenuating. The poor mode quality at the waveguide exit wis caused by imperfections in the waveguide - primarily the placement of the final $90^{\circ}$ bend. At $90^{\circ}$ bends, a spectrum of modes is generated. The modes are attenuated in the following length of waveguide in varying degrees, governed by the attenuation characteristics of the respective modes. If the waveguide is long enough, the only mode that remains is the dominant $E H_{11}$ mode. The generation of a spectrum of modes and the sub- 
sequent filtering of subdominant modes explains the attenuating nature of bends. The last bend is just below the machine, and the no mode filtering takes place. The poor mode quality can impair the focusing through the machine. The $E H_{11}$ mode is similar to a Gaussian beam after exiting the waveguide. Gaussian beams are the most efficiently focused modes, and even Gaussian beam propagation theory predicts the access on ZT-40M to be marginal. The other modes, which do not focus as well, may not propagate cleanly through the machire. If they are lost, it is no different from the waveguide filtering - the machine would act like a spatial filter. However, there are circumstances where these other modes can cause problems, if they reflect off metal walls and return to the main beam (see Section 5.4).

\subsection{Optical Configurations}

\subsubsection{Simple Polarimeter}

The optical configuration for a heterodyne phase-shift polarimeter used for measuring Faraday rotation on ZT-40M is shown in Figure 4.12. In concept, it is identical to the configuration shown in Figure 3.6; however, it is presented here to also show the diagnostic access on ZT-40M, which played a major role in the development required for obtaining useful measurements. The input focusing lens should be chosen to have the waist position somewhat beyond the midplane of the plasma to have optimal insensitivity to vignetting by the exit aperture when refractive bending is caused by the plasma. This is not possible on ZT-40M, where the diagnostic access is so constrained that the beam could not propagate through the machine unimpaired. This had important consequences on the implementation of Faraday rotation measurements on ZT-40M, which will be discussed in Chapter 6 . The focal length of the lens just before the detector should be chosen to match the detector. Typically, the sensitive area of detectors is small, and a short focallength lens (sometimes called a "fast" lens) is required. TPX plastic lenses (see Appendix C) are used for measurements on ZT-40M. The wire grid polarizer, used as a polarization analyzer, is $\mathbf{5 0 0}$ line-per-inch gold deposited on mylar substrate (see Appendix C). At the end of each diagnostic tubulation (I.D. := $0.688 \mathrm{in}$.) is a z-cut quartz vacuum window, chosen because of the low absorption of crystalline quartz at $184.6 \mu \mathrm{m}$, and the vacuum compatibility of quartz. 


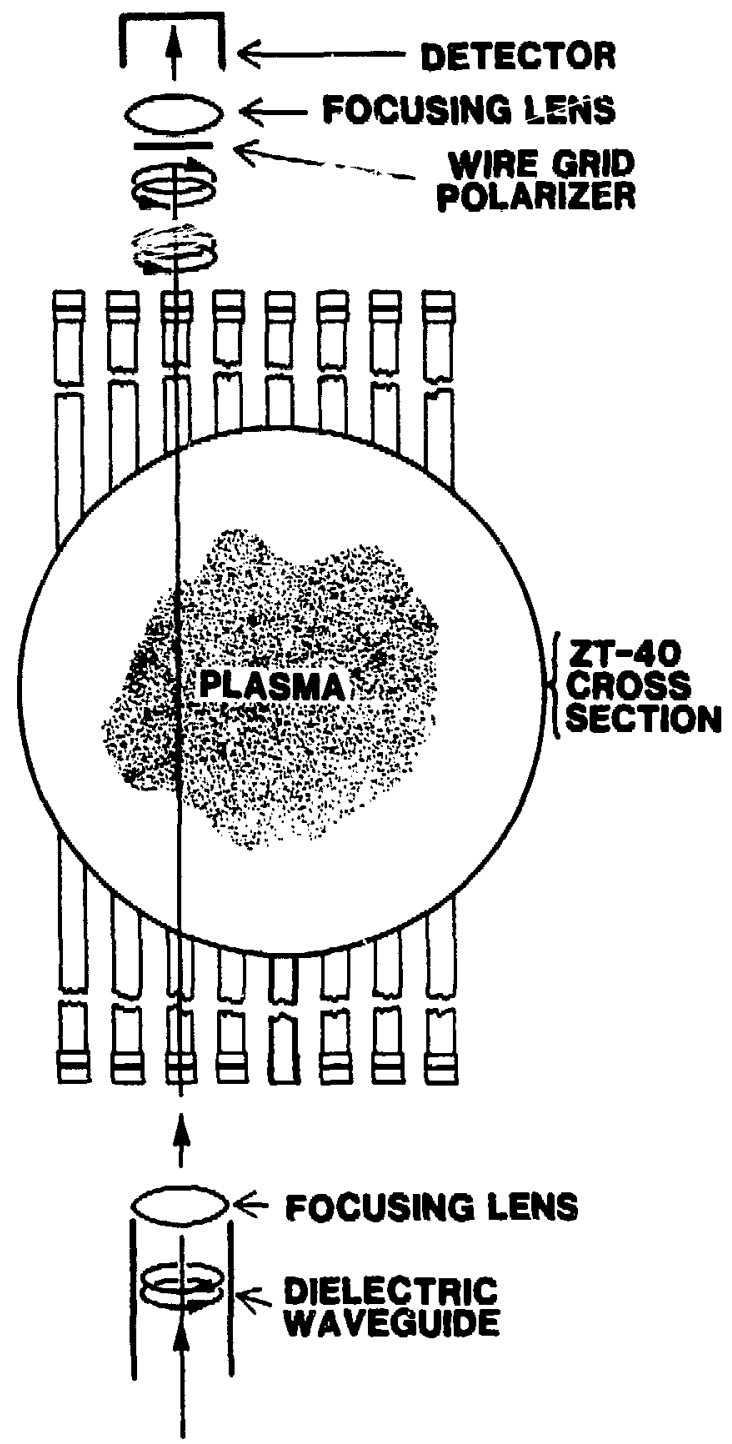

Figure 4.12: Heterodyne Polarimeter on ZT-40M 


\subsubsection{Simple Interferometer}

The optical configuration for a simple interferometer used for density measurements on ZT-40M is shown in Figure 4.13. A Mach-Zehnder configuration was chosen for corivenience - based on the space around ZT-40M, although a Michelson configuration could also have been used. The configuration only requires linear polarization, therefore the $\lambda / 4$ plate beyond the rotating grating in Figure 4.7 is removed for simple interferometry. The orthogonal linear polarizations still need to be shifted in frequency to allow heterodyne phase-shift detection of the plasma refractive index. It is convenient that the different frequency components have different polarizations, because they i : be separated by a polarizing beam splitter. The scene beam of one frequency is sent through the plasma and caused to interfere with the reference beam of the other frequency. The details of the information that can be obtained from this interference are described in Section 3.1.2. The two wire grid polarizers that acted as polarizing beam splitters are 500 lpi gold coated mylar (see Appendix C), and are oriented to completely transmit one polarization and completely reflect the other. Initially the beam splitters were mounted on a simple ring, stretched to make a smooth surface. These are sometimes referred to as pellicle beam splitters. It was discovered quite early that these simple pellicle beam splitters exhibited membrane-like vibrations when the machine fired, causing errors in the interferometric measurements. These vibrations were suppressed by stretching the same material over a TPX plastic fiat. The elimination of the vibrations came at the expense of signal attenuation due to absorption in the TPX. The wire grid polarizer just before the detector is made from the same polarizer material; however, it is oriented at $45^{\circ}$ to the orthogonal linear polarization, and it is here that the scene and reference beams are brought to the same plane and caused to interfere. It does not require a TPX flat since it is only used in transmission.

\subsubsection{Simultaneous Polarimetry and Interferometry}

The optical configuration for a simultaneous measurement of density and Faraday rotation on 2T-40M appears in Figure 4.14. Linear polarizations were propagated through the 17 meters of waveguide to ZT-40M. A non-polarizing beam splitter partitioned the beam between the reference arm and scene arm of the interferometer. A $\lambda / 4$ plate was placed with the axis at $+45^{\circ}$ to the orthogonal linear 


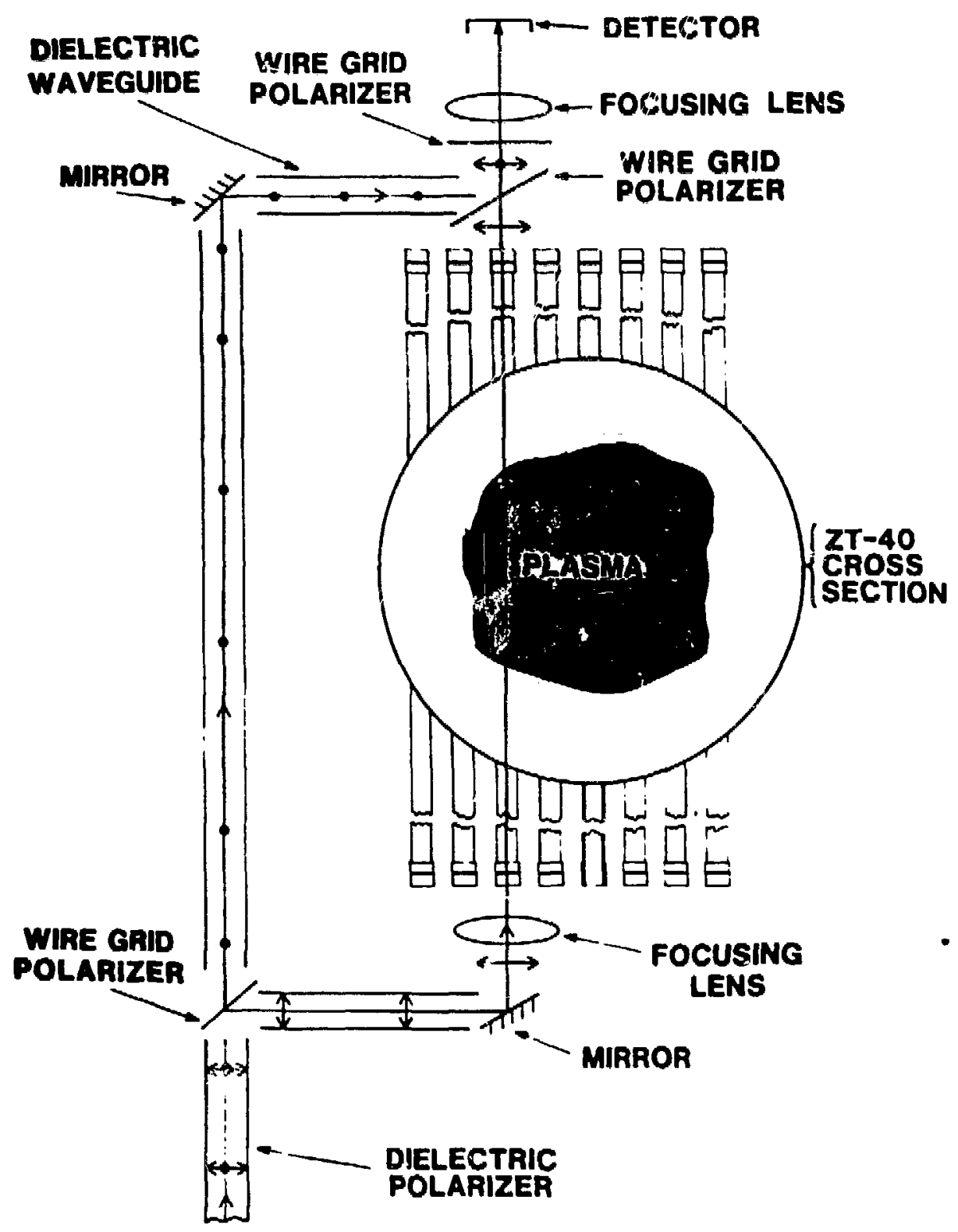

Figure 4.13: ZT-40M Interferometer 


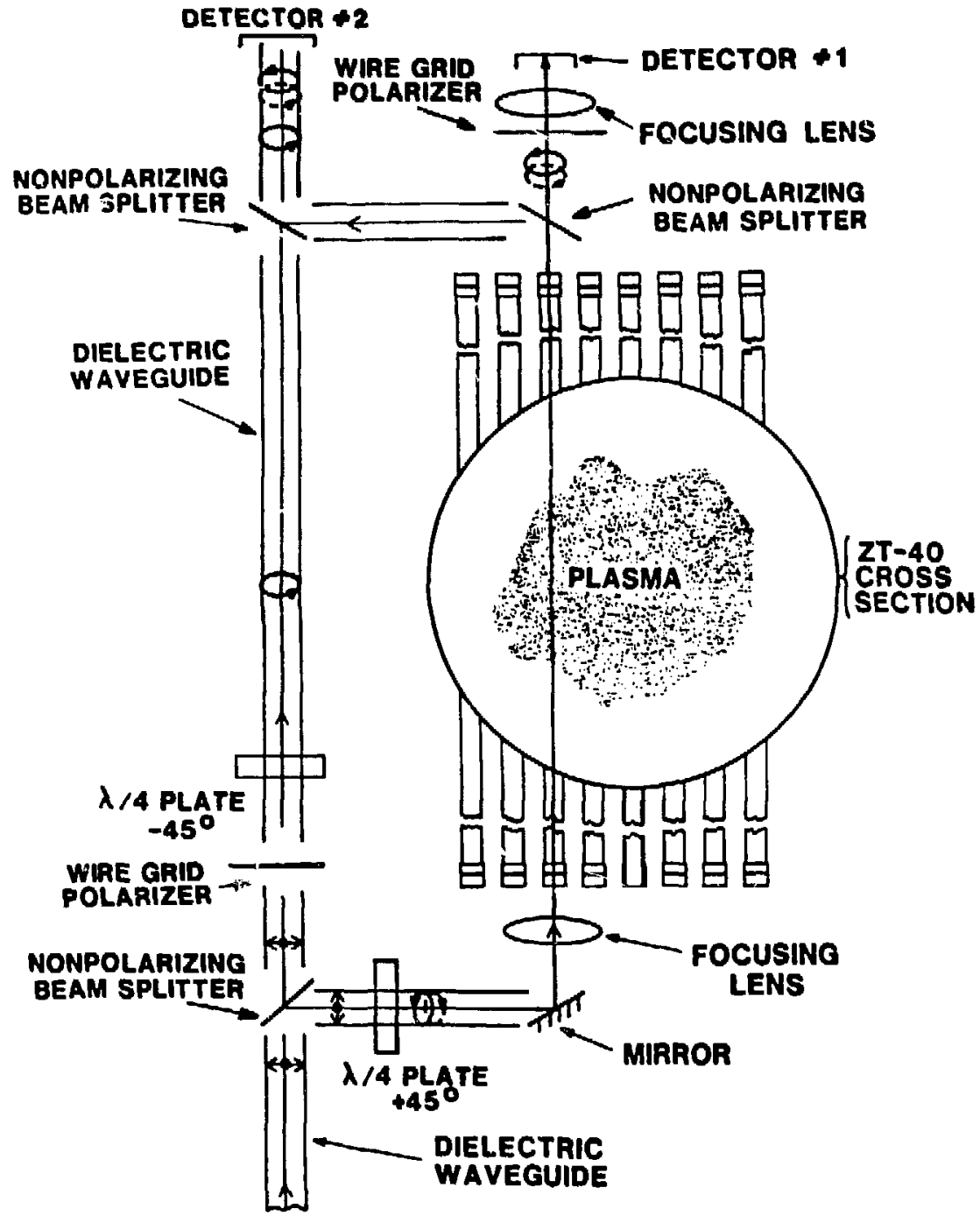

Figure 4.14: Simultaneous Configuration 
polarizations in the scene beam before the plasma, which generated the opposite circular polarizations required for polarimetry. A portion of the scene beam was directed over to interfere with the reference beam for interferometry, and the remainder of the beam was incident on cletector \#1. If one were to unfold the path of the scene beam and ignore the fact that the non-polarizing beam splitters direct some of the beam power elsewhere, the configuration formed by the scene beam and detector \#1 is identical to the simple polarimeter of Figure 4.12. Orthogonal polarizations do not interfere at a square-law detector, so the orthogonal polarizations from the scene beam should produce no signal on detector \#2. The beam of the reference arm was filtered so that only one polarization remains, and the orientation of the $\lambda / 4$ plate in the reference arm was chosen to allow heterodyne interference with one of the polarizations of the scene beam. The reference polarization only interferes with the scene beam polarization of the same sense, and if the $\lambda / 4$ plate is set $-45^{\circ}$, the interfering polarizations will have the frequency difference established by the rotating grating.

There was a serious problem that was discovered in the implementation of the configuration of Figure 4.14. It was found that the orthogonal poiarizations in the scene beam did interfere on detector \#2, indicating that the GaAs detectors were not ideal square-law detectors. Ideal square-law detectors only sense the intensity, which should not vary in time since the scene-beam polarizations are orthogoral. Other detectors sense the electric field of the incident radiation, in which case interference of orthogonal polarizations can result - the detector can act as a mixer of the orthogonal polarizations.

Since the detectors were not ideal square-law detectors, the extraneous polarization from the scene beam needed to be filtered out before it was incident on detector \#2, or errors in the interferometric phase shift determination would have resulted, as the following analysis reveals. The electric field sensed by the detector is given by:

$$
E=A_{I} \cos \left(\omega t+\phi_{I}\right)+A_{E x} \cos \left(\omega t+\phi_{E x}\right),
$$

where $A_{I}$ and $\phi_{I}$ are the amplitude and phase of the interferometric signal, $A_{E x}$ and $\phi_{E x}$ are the amplitude and phase of the extraneous signal, and $\omega$ is the difference frequency established by the rotating grating. Assume for the moment that the quantity of interest, $\phi_{I}$, is determined by measuring the zero-crossing time of the detector signal relative to some reference signal. (Other methods for determining $\phi_{I}$ 
would be subject to similar problems.) The zero-crossing of the signal represented by Equation 4.8 depends on $\phi_{E x}$ and the ratio $A_{E x} / A_{I}$, in addition to $\phi_{I}$. Detectors that are not square-law detectors, require the filtering of the extraneous scene beam polarization. Young $[51]$ chose instead to live with the resultant error, citing that for that particular system, $A_{E x} / A_{I}$ was only $\sim 8 \%$. There is no reason to have to accept this error, however, because the required filtering is quite simple.

The revised optical configuration is shown in Figure 4.15. The filtering takes place at the final polarizing beam splitter, where the extraneous polarizations are directed away from detector \#2. No added optical elements are required for the revised configuration, only the placement of the elements has been changed in order to filter out the extraneous polarization that would otherwise cause errors in the interferometric measurements.

\subsubsection{Multichord Configurations}

All of these configurations can be extended to multichord operation by techniques that are quite simple in concept and easy to understand, but rather difficult to display in a two-dimensional figure. As an example, a two-chord simultaneous configuration is presented in Figure 4.16. The additional chord is made possible by the introduction of an appropriate beam splitter below the machine, altering the path of the reference arm to have the interferometer detector above the exit window, using beam splitters in the reference arm to partition the reference signal to each interferometer detector, and moving the polarimeter detector out of the plane of the paper. One could place the interferometer detectors out of the plane rather than the polarimeter detectors, but this might make the alignment of the interferometer more complicated. Extension to more chords involves the use of more beam splitters above and below the machine.

There is a potential problem in the expansion to multi-chord operation. Crosstalk between channels can result if there is significant back-reflection from the detectors. Quarter-wave plate/polarizer combinations need to be used to isolate these reflecticsis from the system if such problems are observed. 


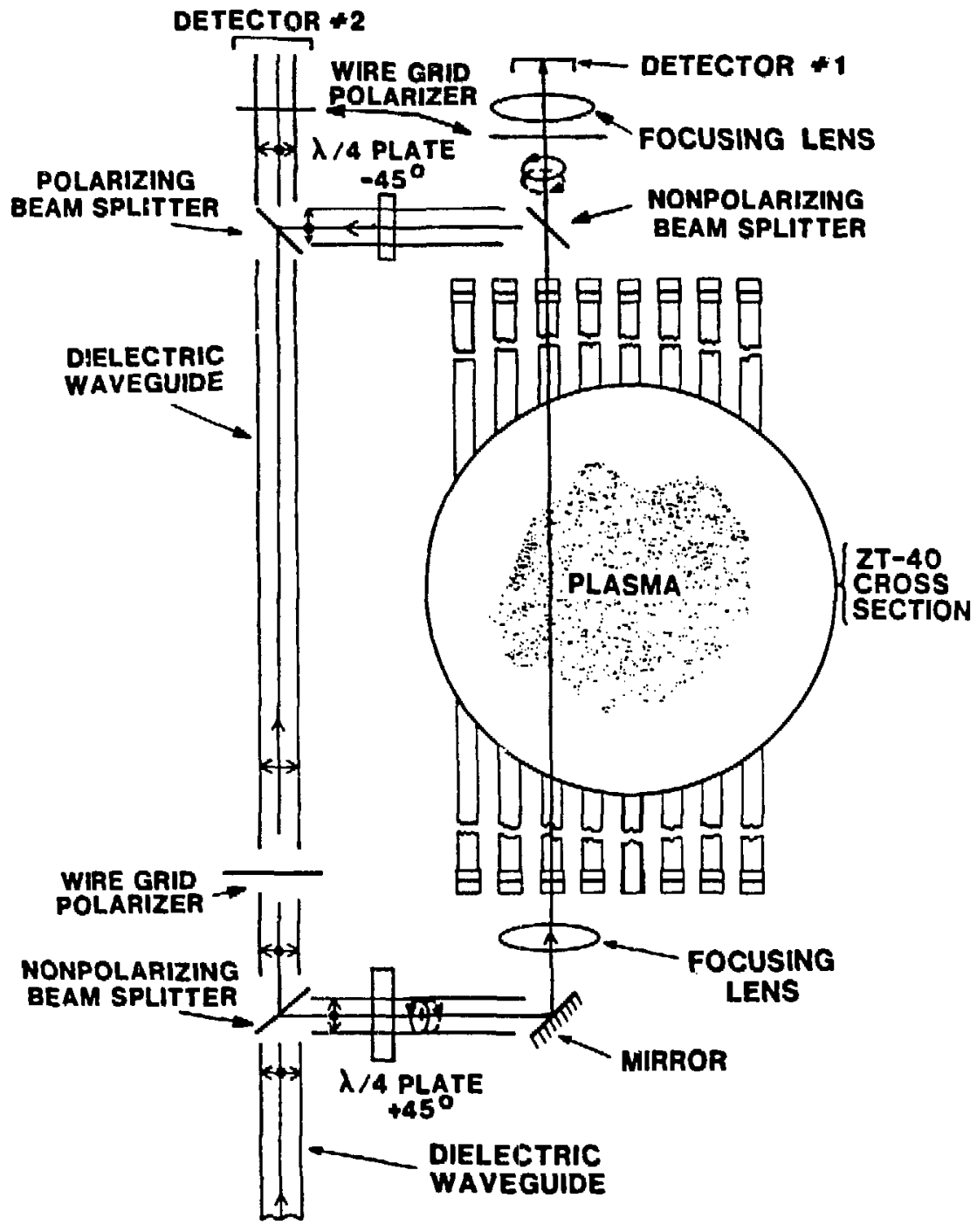

Figure 4.15: Revised Simultaneous Configuration 


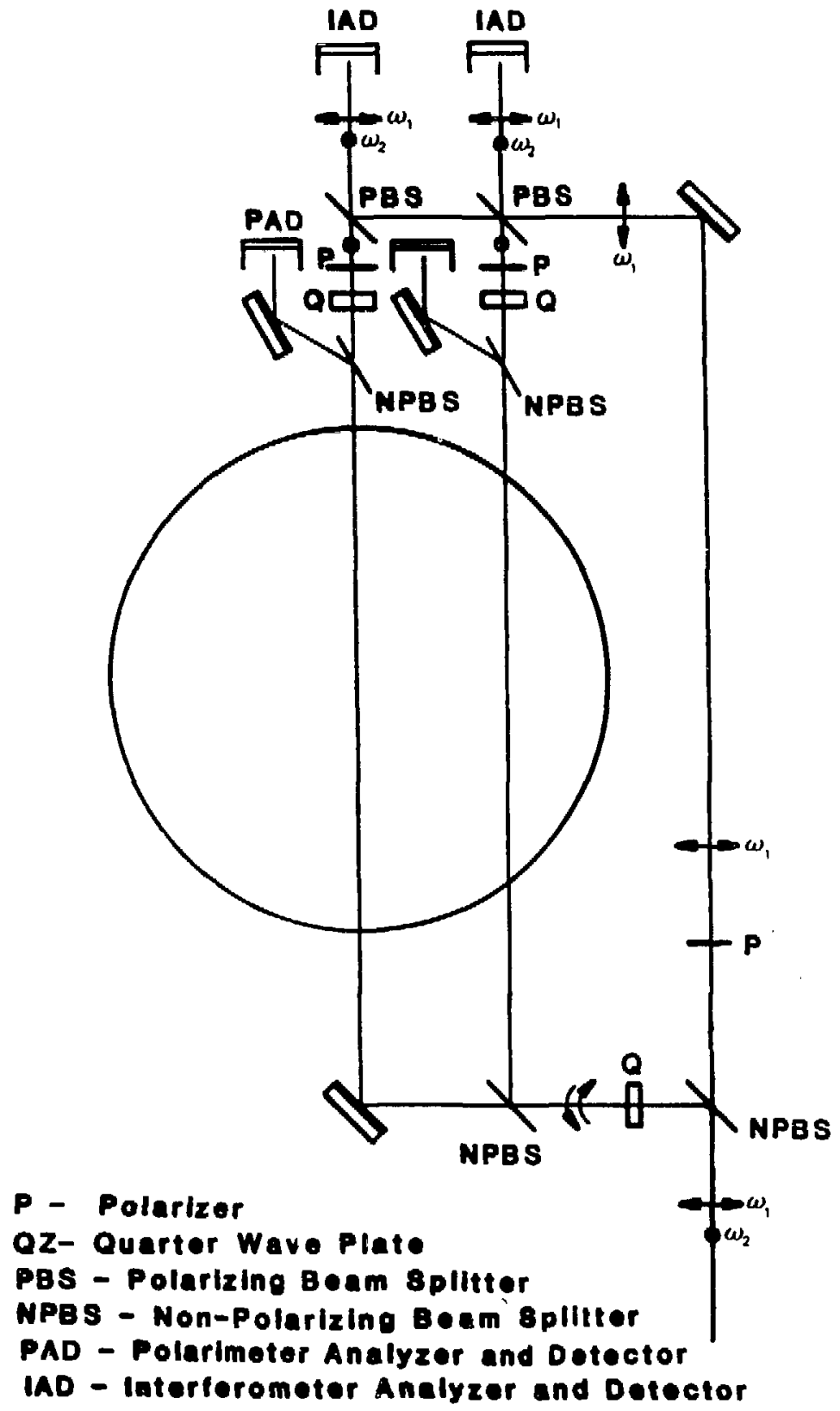

Figure 4.16: Two-Chord Simultaneous Configuration 


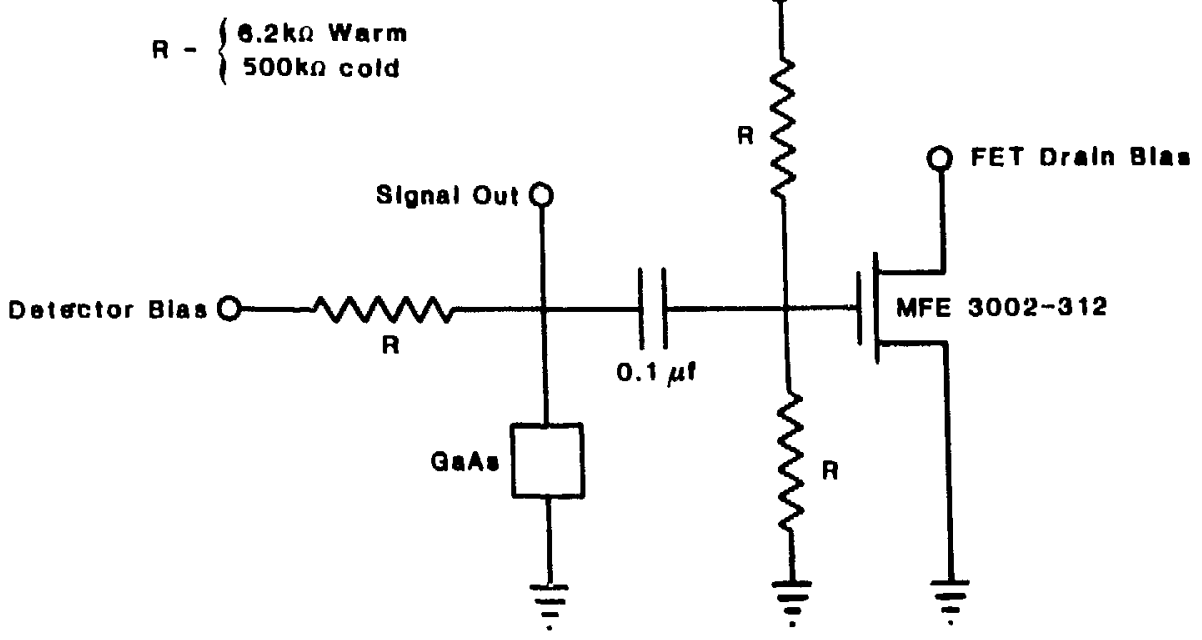

Figure 4.17: Preamplifier Circuit for GaAs Photoconductor

\subsection{Detectors}

\subsubsection{GaAs Photoconductors}

The detectors used for measurements on ZT-40M are epitaxial GaAs photoconductive crystals. GaAs exhibits extrinsic photoconductivity at far-infrared wavelengths, with response time of $\sim 10^{-8}$ seconds [92]. Operation at $4.2^{\circ} \mathrm{K}$ is required to reduce thermal transitions of donor electrons into the conduction band. As a result, the $D C$ impedance of the crystal can be several megaohms at $4.2^{\circ} \mathrm{K}$. Low capacitance impedance matching circuits are required to keep the $R C$ time constant as short as possible. A cryogenic preamplifier is used for the impedance matching [93], and even though care is taken to minimize capacitance, the frequency response of the detector and preamplifier is less than $100 \mathrm{kHz}$. Although some applications would require faster time response, this response is adequate for detecting the heterodyne frequency that is limited by the rotating grating (see Section 4.2.4).

A schematic of the detector preamplifier circuit is shown in Figure 4.17. The cryostat that is used to house the detector and preamplifier is a Model HD-3 Helium Dewar from Infrared Laboratories, Inc. The detectors are mounted on the $4.2^{\circ} \mathrm{K}$ surface with Dow Corning 341 Heat-Sink Compound, which serves as a non-curing adhesive as well as a thermal conductor. The heat-sink compound needs to be 
used sparingly, because the GaAs impedance is a strong function of temperature near $4.2^{\circ} \mathrm{K}$, and significant thermal gradients can cause a drastic reduction in the sensitivity of tbn detector. The preamplifiers are encased in an oxygen-free copper block and mounted to the $4.2^{\circ} \mathrm{K}$ surface of the dewar. Indium gaskets are used to provide a thermally conductive bond to the dewar.

A problem with frequent failure of the FET of the preamplifier was observed. The possibility that thermal cycling might cause this failure motivated the development of a room temperature preamplifier that would not need to be thermally cycled and could be more easily serviced if it failed. The circuit was mounted on the outer wall of the dewar. There was a concern of a degradation of the frequency responce; however, none was observed.

Although the noise equivalent power (NEP) of the GaAs photoconductors has been reported to be $10^{-11}-10^{-12} W H z^{-1 / 2}$, the NEP of the detectors and electronics used on $\mathrm{ZT}-40 \mathrm{M}$ was $10^{-7}-10^{-8} W \mathrm{~W}^{-1 / 2}$. Lower NEP would obviously be desirable, but the detector performance is adequate for the single-chord measurements taken on ZT-40M.

\subsubsection{Other Detector Options}

Due to the inconvenience and operating expense of cryogenic systems, the possibility of using other detectors was investigated. Detectors that have been developed for use in far-infrared systems include Schottky diodes [94] and microbolometers [95]. Far-infrared interferometers, polarimeters, and scattering experiments that have been recently assembled use Schottky diode detectors [70], [43], [34]. An interferometer developed at UCLA for density measur ments on Microtor used microbolometer detector arrays [51]. Such arrays can prove useful for spatial imaging of the detected radiation.

Schottky diode detectors for radiation wavelengths of $\lambda \geq 100 \mu m$ were developed at Lincoln Laboratory [94], and are now available from commercial sources. ${ }^{3}$ The radiation is sensed by a whisker antenna which is mounted at the base of a corner cube. The whisker dimensions are on the order of a wavelength, and therefore a fast imaging system is required for efficient coupling of the radiation to the whisker. The diode junction is formed by a point contact of the whisker to a GaAs semiconductor. Such detectors are reported to have a heterodyne NEP of

\footnotetext{
${ }^{3}$ e.g., Millitech: Amherst, Massachusetts and Farran: Cork, Ireland
} 
$\sim 10^{-18} \mathrm{~W} / \mathrm{Hz}$ and a video NEP of $\sim 10^{-9} \mathrm{~W} / \mathrm{Hz}^{1 / 2}$. The operating frequency of $40 \mathrm{kH} z$ on the ZT-40M system was effectively in the video region, and testing verified the NEP to be close to the video noise specification, if not somewhat higher. At higher heterodyne frequencies, the Schottky diode performance improves significantly Even at this low frequency, the Schottky diodes exhibited sensitivities within a factor of two of that achieved by the $G a A s$ photoconductors. As a result, the Schottky diodes warranted serious consideration for replacing the cryogenic detectors for subsequent measurements.

Microbolometer detector arrays were developed at Caltech by Rutledge and Neikirk [96]. The radiation is detected by sensing the resistance changes in a small segment of bismuth that is heated by the radiation. The radiation is coupled to the bolometer through a bow-tie antenna structure. The bow-tie conductors also function as electrical leads to the bismuth segment. The antenna and bolometer are deposited on a dielectric substrate by photolithographic techniques. One of the limitations on responsivity of the bolometers is thermal conduction to the substrate. Thermal conduction is minimized by fabricating "air-bridge" microbolometers, where the bismuth is suspended between the antenna leads, and does not contact the substrate. The air-bridge microbolometers are mechanically fragile, however. A compromise is found by using a plastic substrate rather than the quartz substrate, which can support the bismuth and offer some thermal resistance.

The microbolometer detector arrays were also tested; however, the results were inconclusive. Sample detectors were supplied for evaluation by D. Rutledge at Caltech. Initial tests indicated that the NEP was superior to that observed with the GaAs photoconductors or the Schottky diodes. However, the responsivity was orders of magnitude below the expected value. Also, a large percentage of the detectors in the microbolometer array failed within a two month period of time. It is possible that the detectors were defective from the onset. The microbolometers have a tremendous advantage in cost over the Schottky diode detectors; therefore, future development and commercialization of these detectors should be regarded with interest.

Present detector development is directed toward making Schottky diode arrays using photolithographic techniques [97]. This combines the sensitivity of Schottky diode detectors with the small package of the microbolometer arrays. The configuration of the Schottky diodes results in a much more rugged detector than the 
microbolometers. Such Schottky diode arrays may very well be the detectors of the future.

\subsection{Signal Processing}

Digital techniques are employed to calculate the phase shifts of the carrier frequency induced by the plasma. Conventional techniques for measuring the phase of the carrier signal involve determining the time of zero-crossing of the sine wave relative to some reference. A slightly more complicated data reduction algorithm is used for analyzing the measurements made on $2 \mathrm{~T}-40 \mathrm{M}$ [98]. Use of this algorithm results in a somewhat better time resolution of the phase measurements, although it is numerically intensive.

The phase determination requires an accurate calculation of the heterodyne frequency, which is performed in a time period just before the plasma discharge. This time period is also used to establish a zero-point of the phase. The phase during the discharge was calculated on a cycle-by-cycle basis using the following prescription:

- 4 equally spaced points are chosen among the digitized time-points of a single sinusoidal period, with the separation between the time-points chosen to have the entire period well spanned, shown as time-points $a$ in Figure 4.18. The phase determination is most sensitive when there are 2.7 chosen points per period.

- From the 4 points, the baseline, amplitude, frequency, and phase determined, and

- The same calculation was performed with the next set of time-points (shown as time-points $b$ in Figure 4.18), as well as subsequent sets, resulting in a determination of the phase of the carrier signal as a function of time.

- The phase of the reference carrier signal was subtracted from the phase of the carrier signal containing plasma information to extract the plasma-induced phase shifts from the measurements.

Use of a phase determining algorithm that "walked" through the sinusoidal carrier signal differs from analogue methods in that each cycle of the heterodyne signal is 


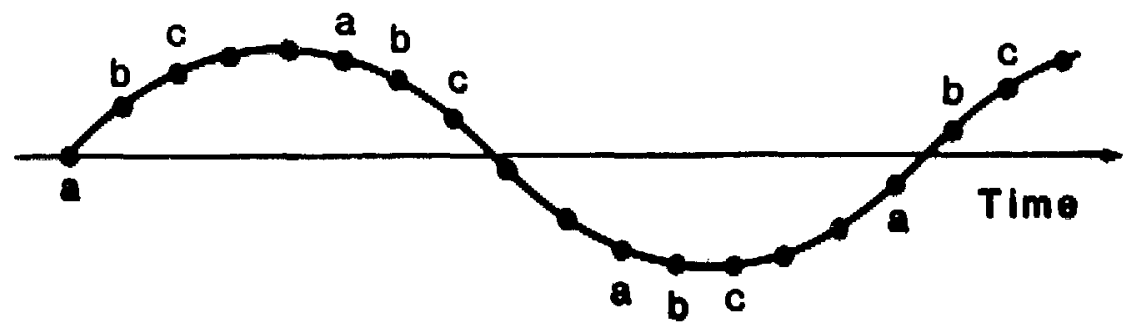

Figure 4.18: Method for Calculating Carrier-Signal Phase

analyzed. It is numerically more intensive, but allows more flexibility in the data analysis.

The present chapter has described the system assembled for polarimetric and interferometric measurements. The radiation source, heterodyning, and beam propagation and detector components were described. Various optical configurations were presented that allow the measurement of chord-averaged Faraday rotation and density separately or ailow the measurement of both quantities simultaneously along the same chord. The signal processing was also discussed briefly, although simplified zero-crossing techniques could also be used, and analogue, sather than digital, methods could be employed in applications where they might be more practical.

The diagnostic development described in this chapter pertains to the implementation of the equipment and techniques on any machine. The following chapter will describe the additional development that was required to make successful measurements on ZT-40M. The major effort was obtaining sufficient polarimetric sensitivity to resolve the small Furaday rotations induced by ZT-40M plasmas. The initial sensitivity limits were caused by factors that are specific to ZT-40M and other constrained-access machines. Chapter 6 will dise uss the interferometric and polarimetric measurements that were actually made on ZT-40M. 


\section{Chapter 5}

\section{Development Required to Obtain Sufficient Sensitivity on ZT-40M}

The previous chapter described the main system components and the development that was required to make each component function properly. The discussions of the previous chapter are relevant to the implementation of such equipment on any device.

That was not the extent of the development required to make useful measurements on ZT-40M, however. There were serious problems in the diagnostic implementation caused by vibration-induced anomalies that were specific to ZT-40M. The development required to overcome these problems may have little bearing on the implementation of such measurements on other devices, unless the diagnostic access has similar characteristics to the access on ZT-40M. Specifically, if the diagnostic access has bare metal surfaces that might reflect even minute portions of the laser beam, problems similar to those encountered on ZT-40M may be observed. There have been other attempts at Faraday rotation measurements that have been abandoned because of problems that could be explained by the effects observed on ZT-40M. These attempts will be discussed in more detail below.

This chapter will describe the development that was required to obtain suffcient polarimetric sensitivity in order to make useful measurements on ZT-40M. In the first section, the measurements that revealed a problem with the polarimetric sensitivity will be presented. The problems occurred during propagation through the machine and appeared to be caused by mechanical vibrations. Measurements indicated that the diagnostic tubulations moved as much as $1 \mathrm{~mm}$ during the discharge pulse. However, it was not clear how that would cause an apparent rotation 
of polarization. The interaction between the beam and the vacuum windows was considered as a possible cause of the problem; however calculations indicated that this effect should be small. Therefore, t'e interaction between the beam and the vibrating tubulations was considered to be the probable cause.

Studies were initiated to determine if the interaction could be avoided by efficient focusing through the machine. Gaussian beam propagation theory predicted that the access apertures on ZT-40M were quite marginal for clean propagation of a beam through ZT-40M. It was found that there is some disagreement in the literature about how severe the effects would be, so a test set-up was assembled to measure the beam propagation characteristics for ZT-40M geometry. Test results agreed with the more pessimistic reference on Gaussian beam propagation, and it was determined that the beam could not propagate through $2 \mathrm{~T}-40 \mathrm{M}$ without interacting with the vibrating tubulation.

Therefore, the only possible solution to the problem was to eliminate the sensitivity of the tubulation interaction. A test set-up was assembled to study the interaction and determine the physical process that was causing the spurious polarization rotation. It was found that a rotation of polarization azimuth was caused by slightly tilting the sample tubulation. The degree of tilting that corresponded to the $1 \mathrm{~mm}$ displacement observed on ZT-40M tubulations caused enough azimuth rotation to explain the anomalous Faraday rotation observed on ZT-40M.

It was considered that grazing metallic reflections off the exposed walls of the tilted tubulations might explain this phenomenon. Sample tubulations that had different inner wall material and surface characteristics were tested. It was found that both quartz walls and internally threaded walls (to baffle against grazing incidence reflections) reduced the sensitivity of tubulation orientation to the azimuth rotation.

A computer program was written to calculate the effects of metallic reflections on the polarization azimuth. The results of the calculations agreed with the levels of rotation observed on ZT-40M and measured in the test set-uF. It was determined that the index of refraction of the metal was large enough that polarization changes occur at grazing incidence, and so even minute fractions of the beam can alter the final azimuth if they reflect off the walls.

Quartz sleeves were installed within the existing ZT-40M tubulations to eliminate the vibration-induced anomaly. The results were disappointing. The level 
of the spurious rotation was reduced, but not by the amount that was consistent with the measurements in the test set-up. More importantly, the reduction was not sufficient to allow a measurement of the plasma-induced Faraday rotation. Upon removal from $\mathrm{ZT}-40 \mathrm{M}$, the reason for the residual problem became apparent. The quartz sleeves had become coated with metal deposits, which altered the optical characteristics of the sleeves. The coating probably started during the discharge cleaning after the vacuum opening when the sleeves were installed. By the time measurements were made, metallic reflection off the coated quartz also caused azimuth rotation.

Internally threaded inserts were then installed within the existing tubulations on ZT-40M. In this case, the coating of the inserts could not affect the performances of the inserts because the metallic reflections were avoided by tailoring the surface to baffle against reflections rather than changing the material composition of the surface. The introduction of the threaded inserts successfully eliminated the sensitivity limits of the polarimeter that was caused by interactions with the vibrating tubulations.

Although identical problems may not be encountered on other machines (they may, in fact, have their own sets of problems), it is instructive to analyze the types of problems that can be found in the implementation of a polarimeter/interferometer. If the diagnostic is being implemented on a machine that may have problems similar to those encountered on ZT-40M, the appropriate preventative steps can be taken from the outset.

Had this measurement been developed on a plasma device designed as a diagnostic test-bed, positive results would have been obtained very early, causing a false impression of the capability for making measurements on the intended machine. The subsequent implementation of an apparently demonstiated diagnostic technique would have required even more development. The implementation of polarimetry/interferometry on ZT-40M has clearly illustrated that a proposed diagnostic technique is not truly demonstrated until it has been applied to a machine environment that is fully representative of the final application.

The remainder of this chapter will discuss in more detail the problems that were encountered on ZT-40M. The experiments that revealed the causes of the problems will be described, and the calculation that verified the physical effects will 
be presented. The changes to $\mathrm{ZT}-40 \mathrm{M}$ diagnostic access that led to the realization of Faraday rotation measurements on $2 \mathrm{~T}-40 \mathrm{M}$ will also be described.

\subsection{Indications of Problems in Polarimetric Sen- sitivity}

\subsubsection{Anomalous Faraday Rotation Caused by Machine Vi- brations}

Even after the developmental steps described in Chapter 4 were implemented, polarimetric sensitivity problems still romained. The improvements that eliminated effects from the imperfections of the rotating grating certainly improved the polarimetric sensitivity to the point that the rotating grating no longer limited the resolution of the polarimeter. Up until that time, the grating effects had overshadowed a completely different phenomenon. The newly revealed problem in sensitivity was characterized by apparent azimuth rotation levels that were larger than the expected Faraday rotation. There was also a unique time variation of the anomalous azimuth rotation, where large modulations of the azimuth would take place on $1-2$ msec timescales. These relatively slow, high amplitude azimuth oscillations extended several milliseconds beyond the time of plasma termination, at which time the oscillations subsided.

The polarimetric anomaly had the character of mechanical vibrations. There are a large number of things that might vibrate during a discharge. However, because of the nature of a heterodyne phase-shift polarimeter, one would expect the detrimental effects of such vibrations to be limited. The following implications were realized:

1. Because a reference detector was used to cancel grating imperfections, the problem had to originate beyond that point, or the reference detector would contain that information as well (recall that the reference detector was located after the grating, just before the beam entered the waveguide used for propagation to ZT-40M).

2. Because the heterodyne method is relatively insensitive to amplitude variations, and the errors did not correlate with the amplitude modulations on the 
carrier signal, the anomalous azimuth rotation had to be caused by anomalous birefringence.

3. The heterodyne method (unlike amplitude modulation methods) can tolerate stray birefringence, as long as the level of birefringence does not vary with time.

Therefore, the polarimetric sensitivity problem had to be caused by stray birefringence that varied during the discharge and was located beyond the reference detector.

The origin of the vibration-induced anomaly was further limited when the measurements shown in Figure 5.1 were taken. Figure 5.1a shows the polarimetric phase measured with the optical configuration of a heterodyne polarimeter (see Figure 4.12), clearly illustrating the sensitivity problem. Figure 5.1b shows the polarimetric phase measured with identical conditions, except that the beam is directed around the torus (along the interferometer reference arm) rather than through the torus and plasma. The implication of Figure 5.1b is that the problem was generated by propagation through $\mathrm{ZT}-40 \mathrm{M}$, rather than vibrations of the waveguide or optics around ZT-40M, or any effects before the reference detector that, for any reason, might not affect the reference detector.

\subsubsection{Possible Causes of Vibration-Induced Anomaly}

Having limited the origin of the problem to propagation through the machine, there were only a limited number of possible causes. Measurements indicated that the diagnostic tubulations were deflected on the order of $1 \mathrm{~mm}$ due to the impulses of the discharge. This was determined by directing a He-Ne laser at a mirror mounted on the tubulation which reflected the beam toward the ceiling. The deflection of the tubulation was found by observing the deflection of the $\mathrm{He}-\mathrm{Ne}$ laser spot on the ceiling and taking into account the iength of the tubulation and the distance from the mirror to the ceiling. ${ }^{1}$ How such deflections of the tubulations could affect the polarization azimuth, however, was not understood. The only components that the beam could interact with (other than the plasma) were the z-cut quartz vacuum

\footnotetext{
${ }^{1}$ The author is grateful for the effort of G.A. Wurden for making this crude, but informative, measurement.
} 


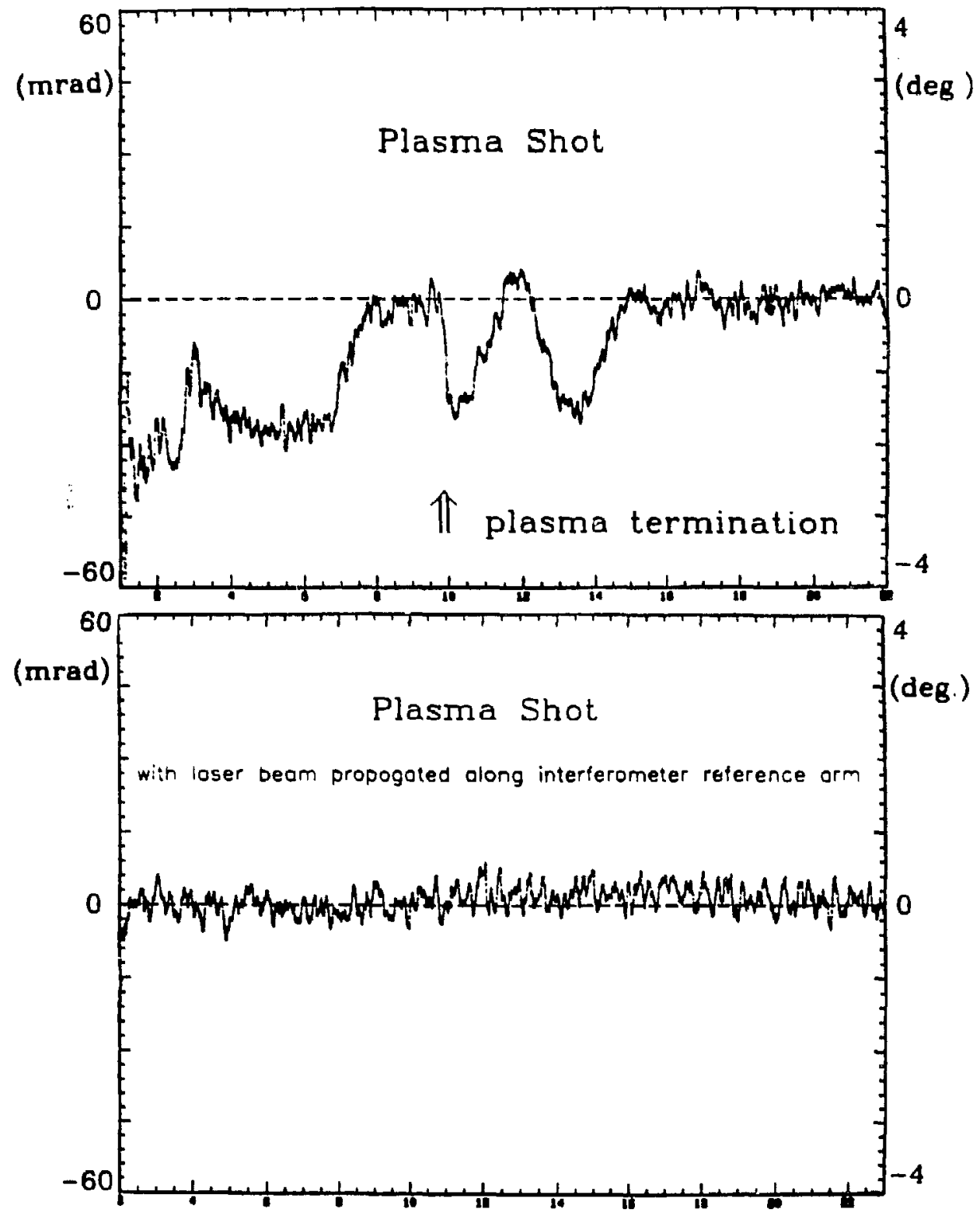

Figure 5.1: Polarimetric Measurements Indicating Sensitivity Probleins 
windows and the metal diagnostic tubulations that comprise the diagnostic access on ZT-40M.

\section{Quarte Windows}

Possible contributions from the z-cut quartz included stress-induced birefringence and geometrical tilts of the $z$-axis during the tubulation vibration period. The stress-induced birefringence effects could be caused by changing stresses in the window due to mechanical vibrations, or by having stress gradients due to vacuum and clamping forces that change the birefringence seen by the beam as the tubulation rocks during a vibration period. The vacuum stresses are compressive in the half of the window that borders atmospheric pressure, and tensile in the half of the window that borders the vacuum. Since the sign of the stress-induced birefringence depends on the sign of the stress, the vacuum stresses should have cancelling effects (to leading order). The clamping stresses may be significant, but do not extend very far into the clear aperture. No measurements of stress-induced birefringent characteristics at far-infrared wavelengths have been made, but theory predicts the effects to decrease at longer wavelengths. Even at visible wavelengths, very large stresses are required to induce measurable effects. Therefore, the possibility of stress-induced birefringence causing the observed polarization rotation was considered to be unlikely.

Geometrical tilts of the quartz window as the tubulation rocks back and forth change the relative orientation of the beam and the z-axis of the quartz. Because crystalline quartz exhibits linear birefringence at beam orientations other than parallel to the z-axis, the observations on ZT-40M could be explained by the changing orientation of the quartz windows as the tubulations vibrated. The equation that governs the orientation dependent birefringence gives the effective phase velocity of the wave in the quartz that has an exiraordinary component [88]:

$$
v_{e f f}^{2}=v_{o}^{2} \cos ^{2} \vartheta \dashv v_{\mathrm{e}}^{2} \sin ^{2} \vartheta
$$

where $v_{0}$ is the ordinary pharc velocity, and $v_{\mathrm{e}}$ is the extraordinary phase velocity. The effective index of refraction is, therefore, given by

$$
\frac{1}{n_{e f f}^{2}}=\frac{\cos ^{2} \vartheta}{n_{o}^{2}}+\frac{\sin ^{2} \vartheta}{n_{e}^{2}}
$$


Using a small angle approximation (for a $1 \mathrm{~mm}$ displacement of the tubulation, $\vartheta=7 \mathrm{mrad}$ ), with $\cos ^{2} \vartheta \simeq 1-\vartheta^{2}$ and $\sin ^{2} \vartheta \simeq \vartheta^{2}$,

$$
n_{e f f} \simeq n_{o}\left[1-\frac{\vartheta^{2}}{2}\left(n_{o}^{2} / n_{e}^{2}-1\right)\right] .
$$

Using the Jones matrix formalism as was done in Section 3.5.2 the maximum phase error caüsed by tilted quartz of thickness $L$ in a heterodyne polarimeter is given by $[99]^{\circ}$

$$
\phi=\left[\frac{\pi L n_{o} \vartheta^{2}}{2 \lambda_{o}}\left(\frac{n_{o}^{2}}{n_{e}^{2}}-1\right)\right]^{2} .
$$

For $\lambda=185 \mu \mathrm{m}, n_{o}=2.117, n_{e}=2.159, L_{o}=3 \mathrm{~mm}, \hat{\vartheta}=.007 \mathrm{rad}$, the maximum phase error is

$$
\phi \sim 1 \times 10^{-8} \text { radians, }
$$

well below the level of phase error observed on ZT-40M.

\section{Metal Tubulations}

Since quartz window effects could not explain the vibration-induced anomaly on ZT-40M, it was concluded that interaction with the metal tubulations was probably the cause of the polarimetric sensitivity problems. In the following sections, calculations and measurements that revealed the effect and led to the solution will be presented.

\subsection{Studies of Beam Propagation for the Geom- etry of ZT-40M}

It was first considered that intersection with the vibrating tubulations could be avoided completely if the beam was focused well through the machine. The vibrating tubulations could have no affect on the bearn if the beam size is much smaller than the tubulation diameter.

Gaussian beam propagation theory (e.g., [100] [101]) was used to calculate the propagation of a $185 \mu \mathrm{m}$ beam through the geometry of 2T-40M. The important parameter for this particular problem is the Rayleigh range. The phys.cal significance of the Rayleigh range is that it is the maximum distance within which a beam of a given size can be focused down and allowed to diverge to its original 
size. If the beam is focused more strongly, the beam waist size is smaller and the beam expands to its original size in a shorter distance. If the beam is focused more weakly, the divergence of the beam results in a relatively large waist size at a shorter distance, and the beam expands to its original size in a distance that is shorter than the Rayleigh range. Using the notation of Siegman [100], the equation for the Rayleigh range is

$$
Z_{R}=\frac{\pi w^{2}}{\lambda}
$$

whe.e $\lambda$ is the wavelength of the beam and $w$ is the spot size (the $1 / e$ radius of the fieid, or $1 / e^{2}$ radius of the intensity) of the initial beam. For the beam to be focused through $\mathrm{ZT}-40 \mathrm{M}$ without interaction with the tubulations it is necessary for $Z_{R}>L$, where $L$ is the separation between the entrance and exit vacuum windows, for a value of $w$ that is much less than the radius of the tubulation.

The other way of approaching the problem is to calculate the value of $w$ by setting $Z_{R}=L$. This determines the smallest allowable spot size at the vacuum windows. For ZT-40M, $L \simeq 63 \mathrm{~cm}$. The corresponding value of $w$ is $6.09 \mathrm{~mm}$. This value cloes not satisfy the condition that $w<<r_{t}$, where $r_{t}$ is the $8.73 \mathrm{~mm}$ radius of the tubulation. Therefore, consideration of the maximum allowable spot size that can propagate through an aperture unchanged became of primary importance.

A rule of thumb is presented by Siegman [100] for the spot size limit for negligible distortion of the transmitted profile, which is given by

$$
w<\frac{2 r_{t}}{3}
$$

where $r_{t}$ is the aperture radius. For the apertures and window spacing on ZT-40M, this condition is slightly violated, with $w=0.70 r_{t}$. If the spot size were made smaller, in order to satisfy the condition of Equation 5.7, then the Rayleigh range is $Z_{R}=57.1 \mathrm{~cm}$, slightly less than the window separation on ZT-40M.

Some added uncertainty was introduced when it was discovered that there is some disagreement in the literature concerning the maximum allowable spot size that can be propagated through an aperture with no profile distortion. The rule of thumb given by Gaskill $[101]$ is

$$
w<\frac{r_{t}}{\sqrt{\pi}}
$$

It is not clear how much analysis went into either condition - they were both justified by statements that if $99 \%$ (or $99.8 \%$ ) of the beam is not clipped by the 
aperture, the effects must be small. However, the condition of Siegman predicts only slight problems with the geometry of ZT-40M, while the condition of Gaskill predicts much more severe problems. The Rayleigh range if the Gaskill condition is satisfied is only $Z_{R}=40.8 \mathrm{~cm}$, which is much less than the window spacing on ZT-40M.

\subsubsection{Computer Calculations}

Because of these uncertainties, testing was warranted to determine the extent of the problems in focusing the beam through $\mathrm{ZT}-40 \mathrm{M}$ without interacting with the tubulations. First, calculations were performed to determine the profile effects of propagating a Gaussian beam through apertures that correspond to the vacuum windows on ZT-40M. A computer code developed at Los Alamos for beam propagation studies for laser isotope separation [102] was used. The results of this study are shown in Figure 5.2. A beam with $w=6.09 \mathrm{~mm}$ is initiated with the radius of curvature for the beam to focus and re-expand (in the absence of apertures) in a distance corresponding to the Rayleigh range. That beam is then clipped by an aperture corresponding to the entrance window on ZT-40M. The beam is propagated to the exit aperture, where it is again clipped. Figure 5.2a shows the beam after it is clipped by the entrance aperture. The clipping is seen to have only slight effects on the tails of the Gaussian distribution, and $98 \%$ of the beam is transmitted through the aperture. Figure $5.2 \mathrm{~b}$ shows the beam profile at the theoretical waist position, at a distance corresponding to the mid-plane of ZT-40M. Figure 5.2c shows the beam after being clipped slightly by the exit aperture, and that $96 \%$ of the original beam intensity is transmitted through the machine. Although the profile effects are very slight, propagation through an additional $30 \mathrm{~cm}$ pathlength shows Fresnel diffraction effects. These result from the interference between the main berm and the portion of the beam that diffracts off the edges of the aperture.

\subsubsection{Experimental Measurements of Beam Propagation}

Even though the computer calculations indicated only minor profile effects due to the apertures of ZT-40M for this idealized case, the focusing of a non-Gaussian profile would not be as efficient. In addition, the vibrating tubulations move as much as $1 \mathrm{~mm}$ during a discharge, which causes the effective apertures of the 

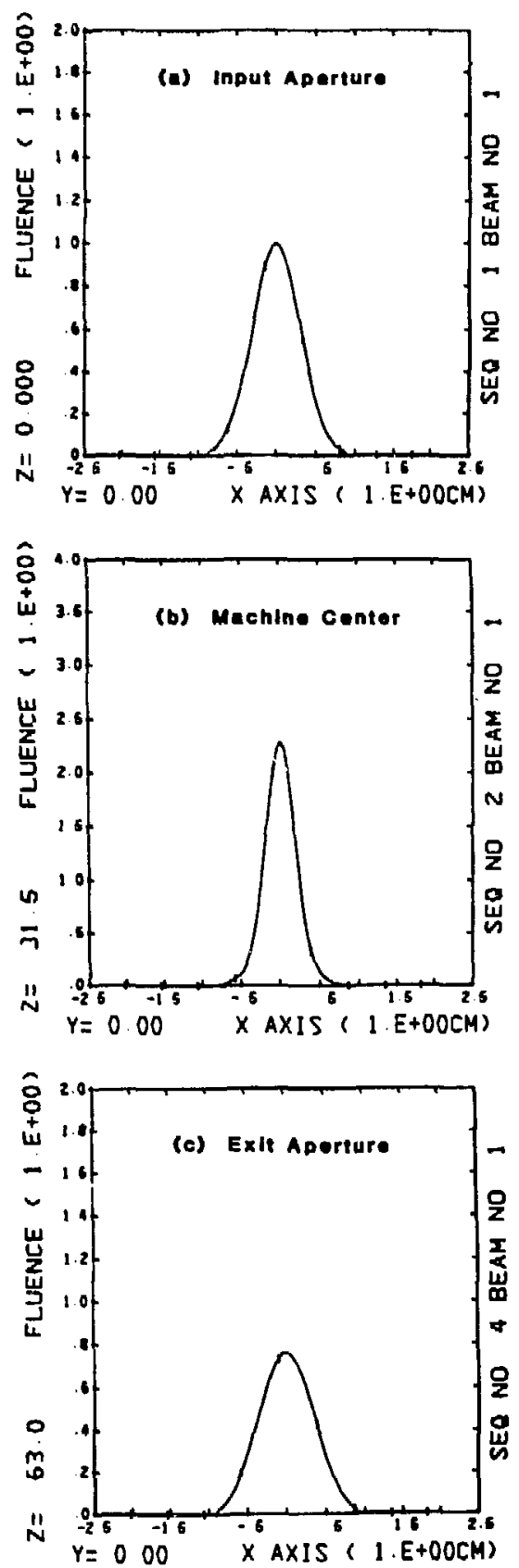

Figure 5.2: Theoretical Beam Profiles for Geometry of ZT-40M 


\section{FIR Laser}

\section{Waveguide}

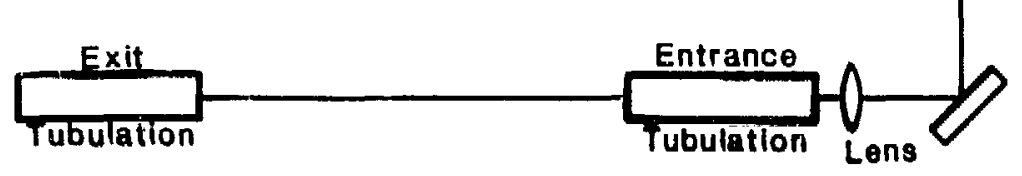

Figure 5.3: Set-up for Beam Propagation Measurements

windows to be smaller. To determine the effects of these additional factors, an experimental test set-up was assembled to measure the beam propagation on ZT40M under realistic conditions.

A schematic of the experimental set-up appears in Figure 5.3. The laser beam was propagated through a 1.5 meter length of hollow dielectric waveguide. This provided a mode-filtering function, and simulated the conditions that were probably more representative of those on the real ZT-40M system, where a waveguide was used to propagate the beam to the machine. The beam was then allowed to expand to the proper spot-size, and a lens was used to give the beam the appropriate radius of curvature according to Gaussian beam propagation theory. The beam was propagated through a distance corresponding to the separation between vacuum windows on ZT-40M, and a comparison was made of the profiles with and without the tubulations inserted in the beam. The measured intensity profiles appear in Figure 5.4. The traces on the left side of the figure represent the profile at the machine center position and at the exit position, with no tubulations in the beam. The traces on the right show the profile measurements at the same positions, when the tubulations were in place. The first thing to notice is that the peak intensity at the machine center position was increased by introducing the tubulations. This is a result of constructive interference between the main beam and the portion of the beam that has diffracted off the edges of the aperture. This effect is known as 


\section{Beam at Machine Center Position}
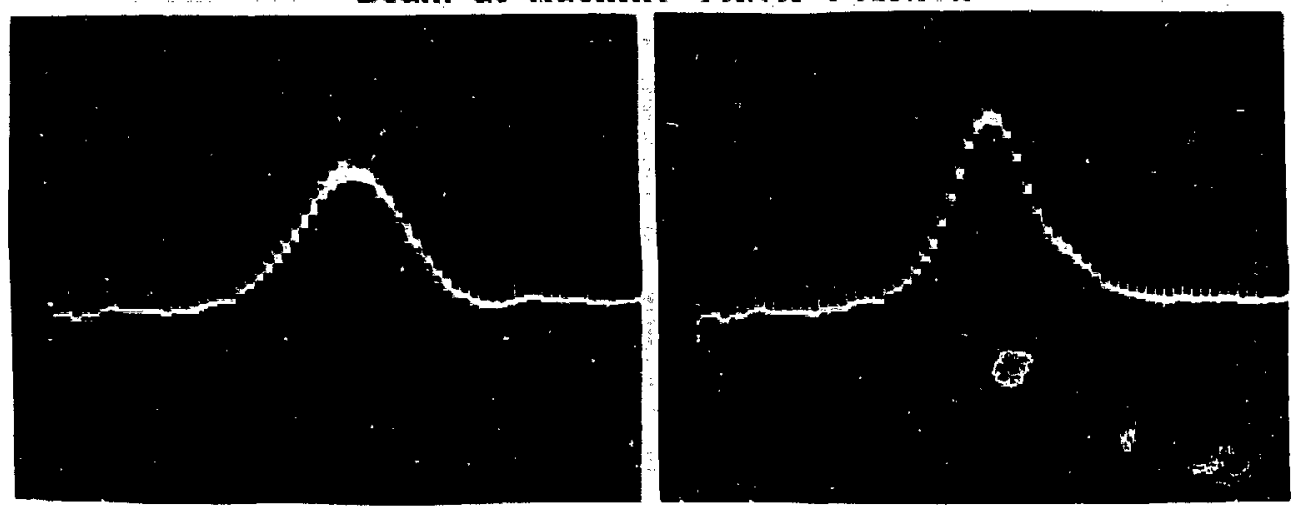

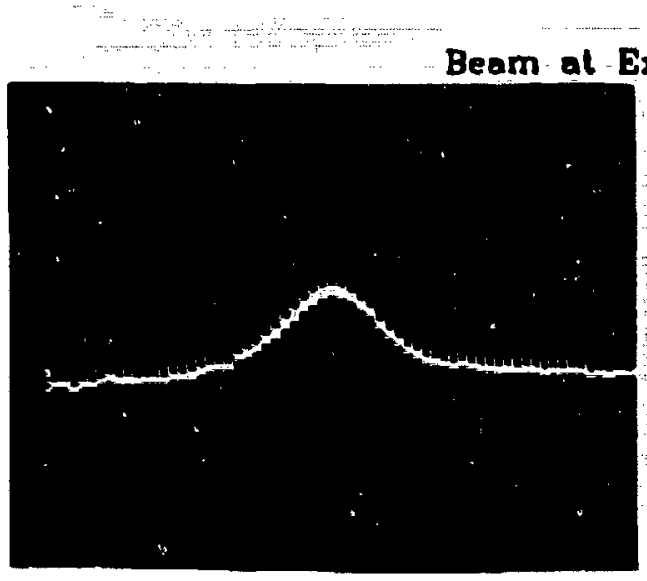

Without Tubulations

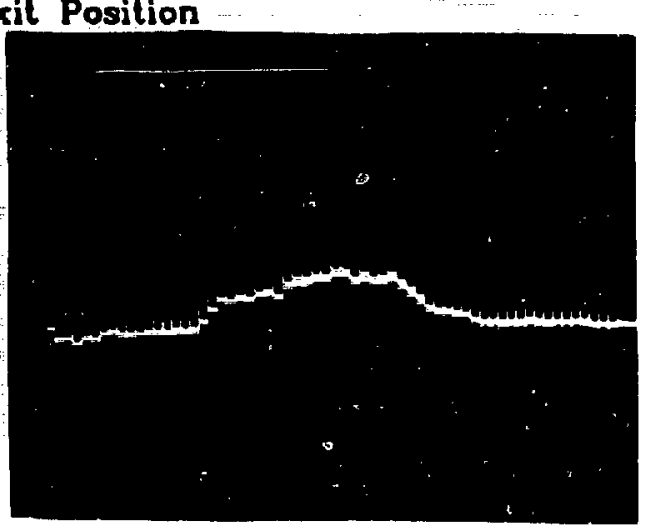

With Tubulations

Figure 5.4: Measured Intensity Profiles for Geometry of ZT-40M 


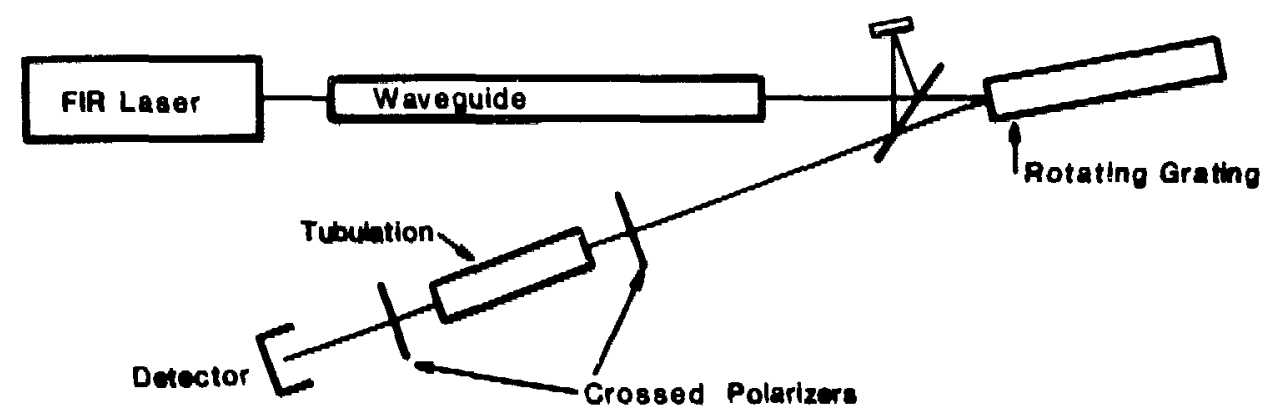

Figure 5.5: Crossed Polarizer Test Set-up

Fresnel focusing. This is sometimes exploited in applications where it is necessary to maximize the peak intensity of the beam over what is possible with Gaussian propagation, when the resultant loss of integrated intensity is not important. For focusing in ZT-40M, however, the Fresnel focusing causes more severe problems downstream. The spot size is smaller at machine center, which means that the beam expands more before the exit aperture is reached. This is why the severe clipping is observed at the exit position when the tubulations are in place. The implication of the Fresnel focusing and the more severe clipping was that the interaction between the beam and the vibrating tubulations on $\mathrm{ZT}-40 \mathrm{M}$ was unavoidable for realistic conditions.

\subsection{Measurement of Tubulation Birefringence}

Since optimal focusing of the beam through the machine could not be expected to eliminate the interaction between the beam and the vibrating tubulations, efforts turned to determining methods of eliminating the effects of the interaction.

$A$ test set-up was assembled to measure the tubulation birefringence and try to determine the cause of the birefringence. A schematic of the test set-up appears in Figure 5.5. The sample tubulation was placed between crossed polarizers, with adjustable mounts to allow variability of the tubulation orientation. A Schottky 
diode was used as a detector, and a rotating grating was employed to allow the measurements to be made at a high frequency, which reduced the $1 / f$ noise of the detector. A lock-in amplifier was used to improve the sensitivity of the measurements.

The orientation of the tubulation was varied to simulate the positions the tubulations on ZT-40M might take on during a vibration period. Any signal that was detected beyond the crossed polarizers had to be caused by polarization changes generated by the sample tubulation. The effects that would result in signal on the detector are depolarization, elliptization, and azimuth rotation. Depolarization and elliptization were not of primary interest because the heterodyne phase shift method employed on ZT-40M is insensitive to these effects. Azimuth effects were isolated by determining if there was a new orientation of the second polarizer that produced a minimum transmitted signal.

The azimuth rotation generated by the tubulation was measured as a function of tubulation orientation. Significant rotation was indeed observed with a tubulation similar to those that comprise the diagnostic access on ZT-40M. It was surmised that the effect could be altered if the refiections off the tubulation were eliminated. This was easily accomplished by machining internal threads on the tubulation walls. The threaded tubulation was then tested between the crossed polarizers. A quartz tubulation was also tested to see if different optical properties of the tubulation walls would alter the effect.

The results of the testing are shown in Figure 5.6. The graph shows the polarization azimuth rotation observed as the exit end of the tubulation was translated and the entrance end remained fixed, which generated a tilt angle between the beam and the tubulation. The bare metal tubulation exhibited strong azimuth rotation as a function of orientation - consistent with the level of anomaly observed on ZT-40M - while the quartz and threaded tubulations suppressed the rotation by an order of magnitude.

\subsection{Explanation of Tubulation Birefringence}

It was thus shown that the variable tubulation birefringence originates in the physics of metallic reflection. It can be explained as follows: a beam incident on a metal surface will experience different reflectivities and different phase shifts 


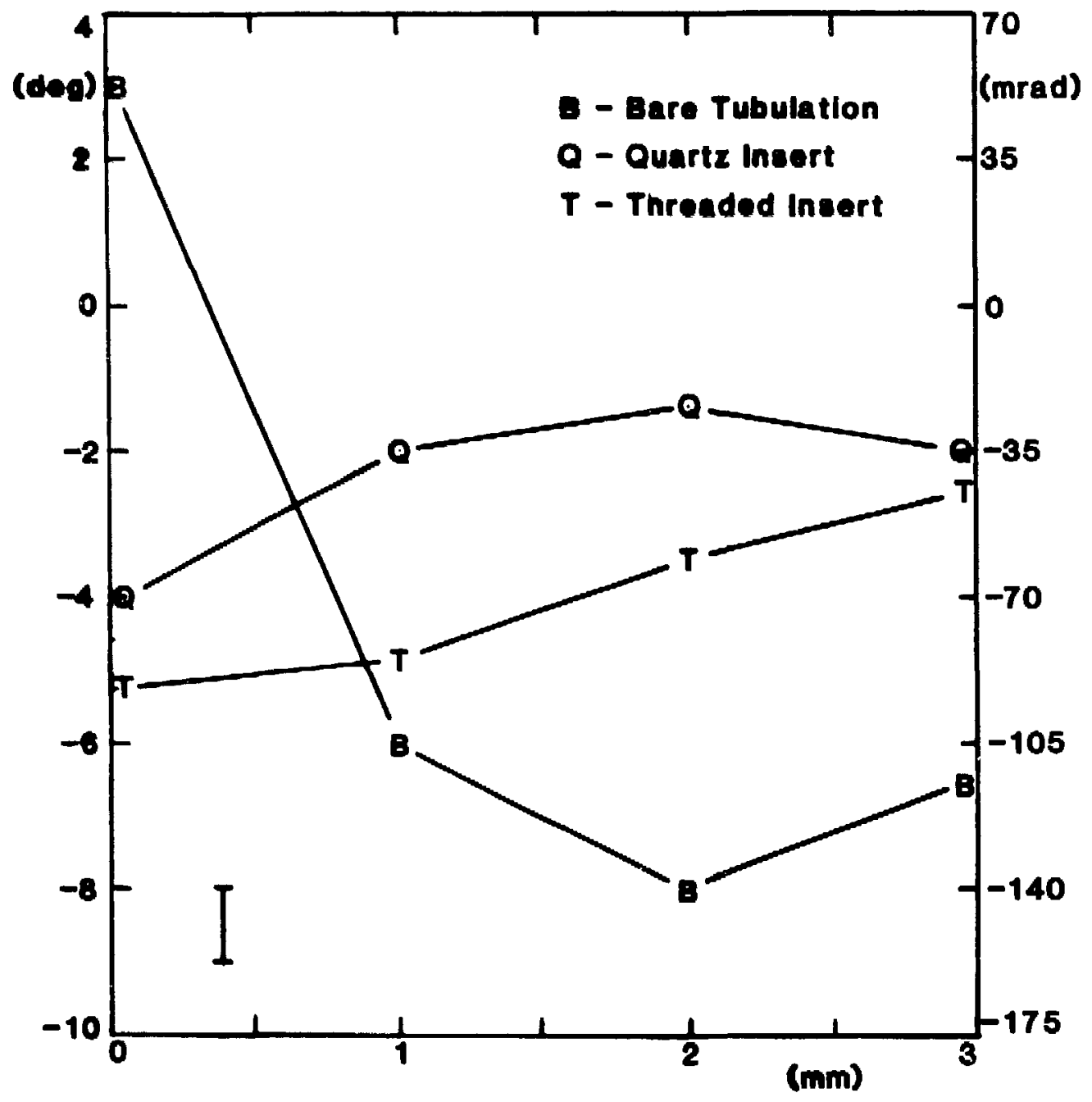

Figure 5.6: Polarization Azimuth Rotation for Bare Metal, Quartz, and Threaded Tubulations 
for the $s$ and $p$ components of the electric field ( $s$ polarization is perpendicular to the plane of incidence and $p$ polarization is parallel to the plane of incidence). This causes the reflected beam to be elliptical in polarization, and the major axis of the ellipse to be rotated. Calculations were performed to determine if the metallic reflection effect could explain the level of birefringence observed when the tubulation is at a slight angle to the laser beam. A quick calculation indicated that a large enough fraction of the beam is intercepted by the exposed wall of the tubulation to alter the final polarization if severe polarization changes take place upon reflection.

A more detailed calculation was required to take into account the complicated geometry of the tilted tubulation. Using the formalism set forth by Born \& Wolf[88], the index of refraction of Inconel was calculated to be $65+i 65$ for $185 \mu \mathrm{m}$ radiation. The index of refraction being complex causes the angle of reflection to be complex, which is an indication that phase changes occur upon reflection. The resulting reflectivities of the $s$ and $p$ components and the phase difference between the reflected components are plotted against incident angle in Figure 5.7. The ratio of the reflectivities and the phase difference between the two components are strong functions of incident angle for grazing incidence. Both of these effects contribute to the alteration of the polarization upon reflection.

A computer program was written to calculate the effects on the final polarization from these metallic reflections. The model assumes a plane wave is incident on the entrance aperture of the tubulation, and that all of the energy appears at a detector beyond the tubulation exit. No divergence or diffraction effects are considered, that is, geometric optics are assumed throughout. The incident beam is linearly polarized at a given azimuthal angle, and is resolved into $x$ and $y$ components. The bearn is segmented into elements, and the elements that are not intercepted by the tubulation wall are propagated through unchanged. The elements that do suffer reflection are transformed into the s-p coordinate system, where the Inconel optical constants can be applied. After reflection, an elliptical polarization is approximated as a linear polarization that is aligned with the major axis of the ellipse. The $s$ and $p$ components are then transformed back to the $x-y$ coordinate system and added to their respective components of the final beam. The azimuth of the final beam is then compared to that of the original beam. Of interest is the difference between the initial and final polarization azimuths, which represents the birefringent effect of the tubulation. 


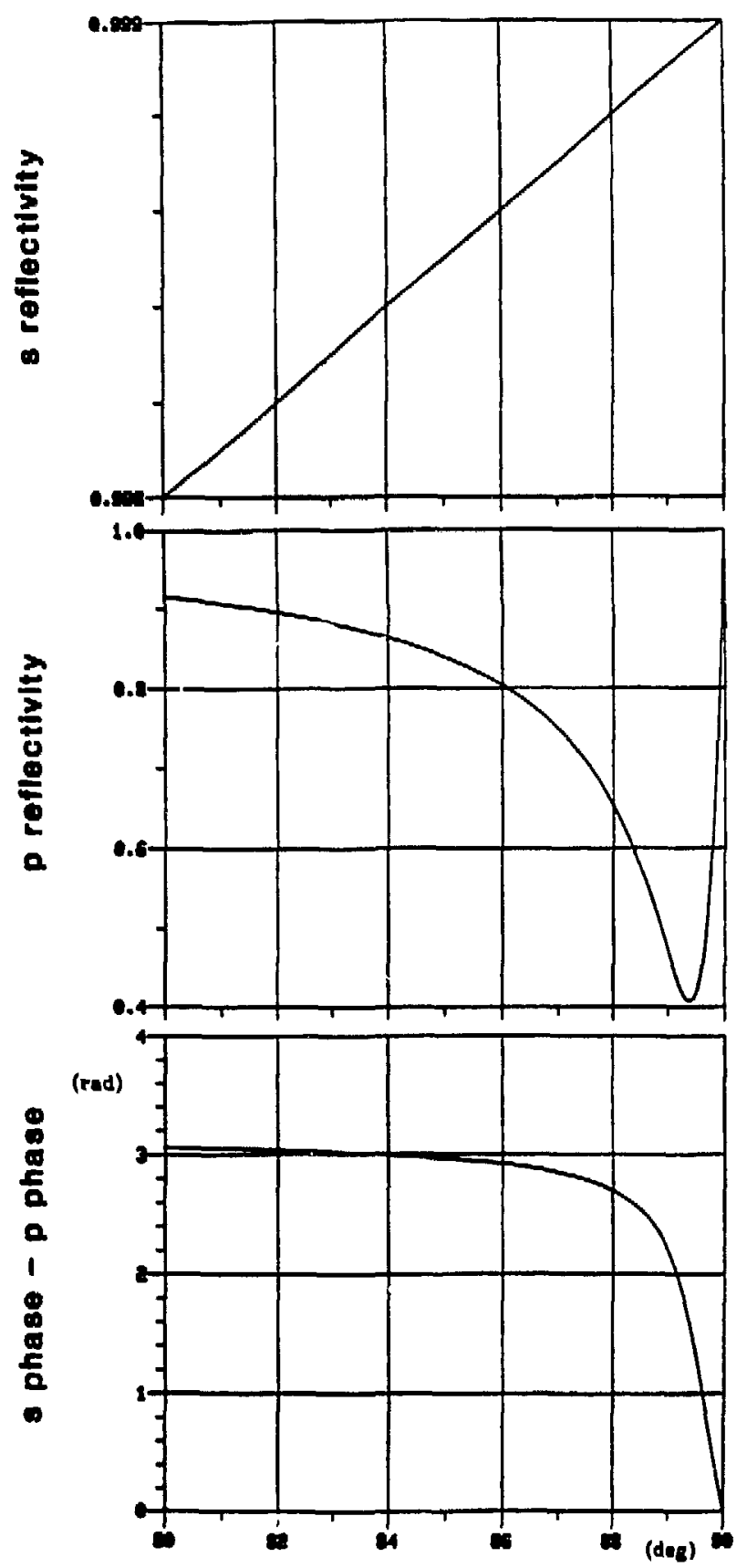

Figure 5.7: Reflectivities and Phase Difference for $s$ and $p$ Polarizations for Grazing Angles of Incidence 
The results of the calculation are shown in Figure 5.8. The difference between the initial and final azimuth is plotted as a function of initial azimuth for several values of exit aperture displacement. Inspection of Figure 5.8 reveals that the theory predicts that the azimuth rotation changes sign as the tubulation angle is changed, as was observed in Figure 5.6 (the measurements were taken with an incident azimuth of $45^{\circ}$ ). It is not clear how much confidence should be placed in a detailed comparison, given the simplified nature of the theoretical model. The levels of rotation predicted by the calculations do, however, agree very well with the crossed polarizer measurements and observations on ZT-40M when it is remembered that the basic method for measurements involves the use of a constantly changing incident polarization azimuth. Because the heterodyne technique averages over incident azimuth, the observations on ZT-40M could only be explained if the tubulations were moving. (For amplitude polarimetry methods, errors would result even if the tubulations were stationary, because the Faraday rotation induced by the plasma changes the polarization azimuth incident on the exit tubulation. As a result, the amount of tubulation-induced rotation changes as the amount of Faraday rotation changes, and the determing the actual Faraday rotation level is difficult.)

The bare tubulation exhibited orientation-dependent birefringence because the optical properties were strong functions of angle at grazing incidence. The threaded sample suppressed the birefringence by baffling against metallic reflections. The reason that the quartz sample did not exhibit orientation-dependent birefringence is because of the dielectric properties of quartz. The index of refraction is only $\sim 2$ rather than $\sim 60$, and the optical properties of the quartz are not a strong function of angle at grazing incidence.

\subsection{Modifications to ZT-40M to Reduce Sensi- tivity to Vibrations}

Using the results of the measurements that indicated that metal reflections were causing the vibration-induced anomaly, it was decided that modifications to the tubulation walls would reduce the polarimetric sensitivity to vibrations. Both the quartz and threaded sample tubulations suppressed the orientation-dependent 

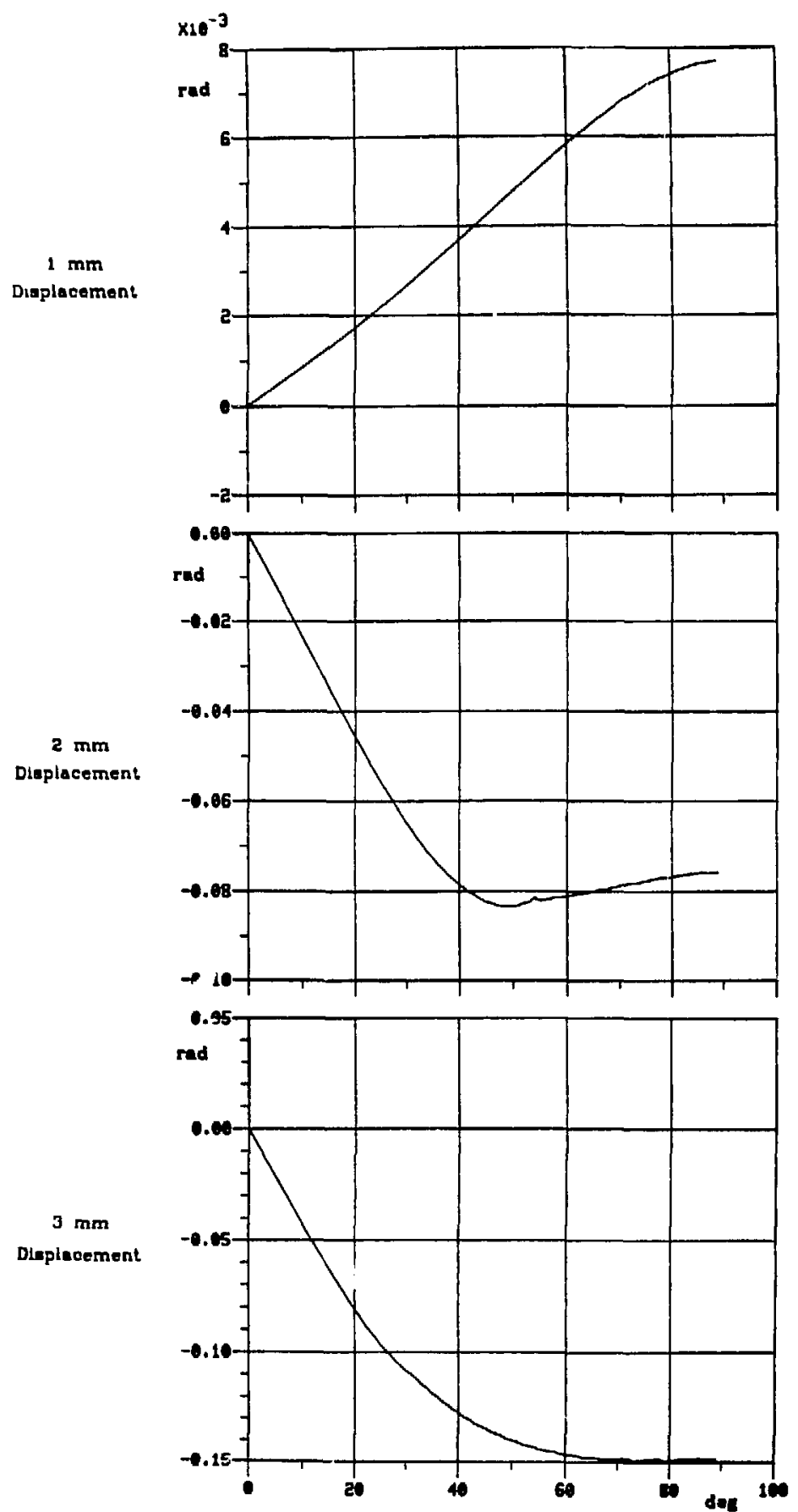

Figure 5.8: Polarization Rotation vs. Incident Azimuth 
birefringence. Therefore, inserting either quartz or threaded walls within the tubulations should eliminate the polarimetric sensitivity problems.

\subsubsection{Quartz Inserts on ZT-40M}

Quartz inserts were installed within the existing tubulations on ZT-40M. The threaded inserts suppressed the birefringent effects by baffling against reflections, and the radiation that was incident on the tubulation walls was effectively lost. On the other hand, the quartz inserts suppressed the birefringent effects by having dielectric properties that were not strong functions of angle at grazing incidence. Therefore, with the quartz inserts, the radiation that was incident on the tubulation walls was not necessarily lost. The only possible advantage of the quartz over the threaded inserts was that the quartz may not have attenuated the beam as much.

The quartz insert diameter was chosen to allow sufficient clearance between the quartz and the existing tubulations. The inserts were mounted by bonding (TorrSeal ${ }^{\oplus}$ ) a metal ring around the outer diameter of the quartz at the window end of the insert, and modifying the vacuum window mount to hold the ring. The end of the quartz insert that faced the plasma was free-standing. Inserts were installed on the top and bottom tubulations of the central chord, and the top and bottom of the edge chord at $R=R_{o}+12.4 \mathrm{~cm}$.

The effect of the quartz inserts on the polarimetric sensitivity was disappointing. The anomalous azimuth rotation was reduced, but only by a factor of $\sim 2$. Some weeks later, during a very high current, short pulselength run on ZT-40M, one of the top quartz inserts broke. This was probably a result of the impulses $o \hat{i}$ the discharges causing larger deflections than were allowed for. (Incidentally, the quartz sleeve was not the only casualty of the high current run. One of the graphite shields that protect large tubulation welds had inched its way into the plasma, and the Thomson scattering viewing dump was dislodged and lying in the bottom of the vacuum chamber.) Fortunately, the quartz insert that broke remained in one piece, which made the retrieval relatively simple.

The other quartz sleeves were also removed. They appear in Figure 5.9, photographed after their removal. Upon inspection, the reason for the disappointing results were obvious. The quartz sleeves had been extensively coated with metal deposits, even though they had only been in the machine for $\sim 1000$ shots. Con- 


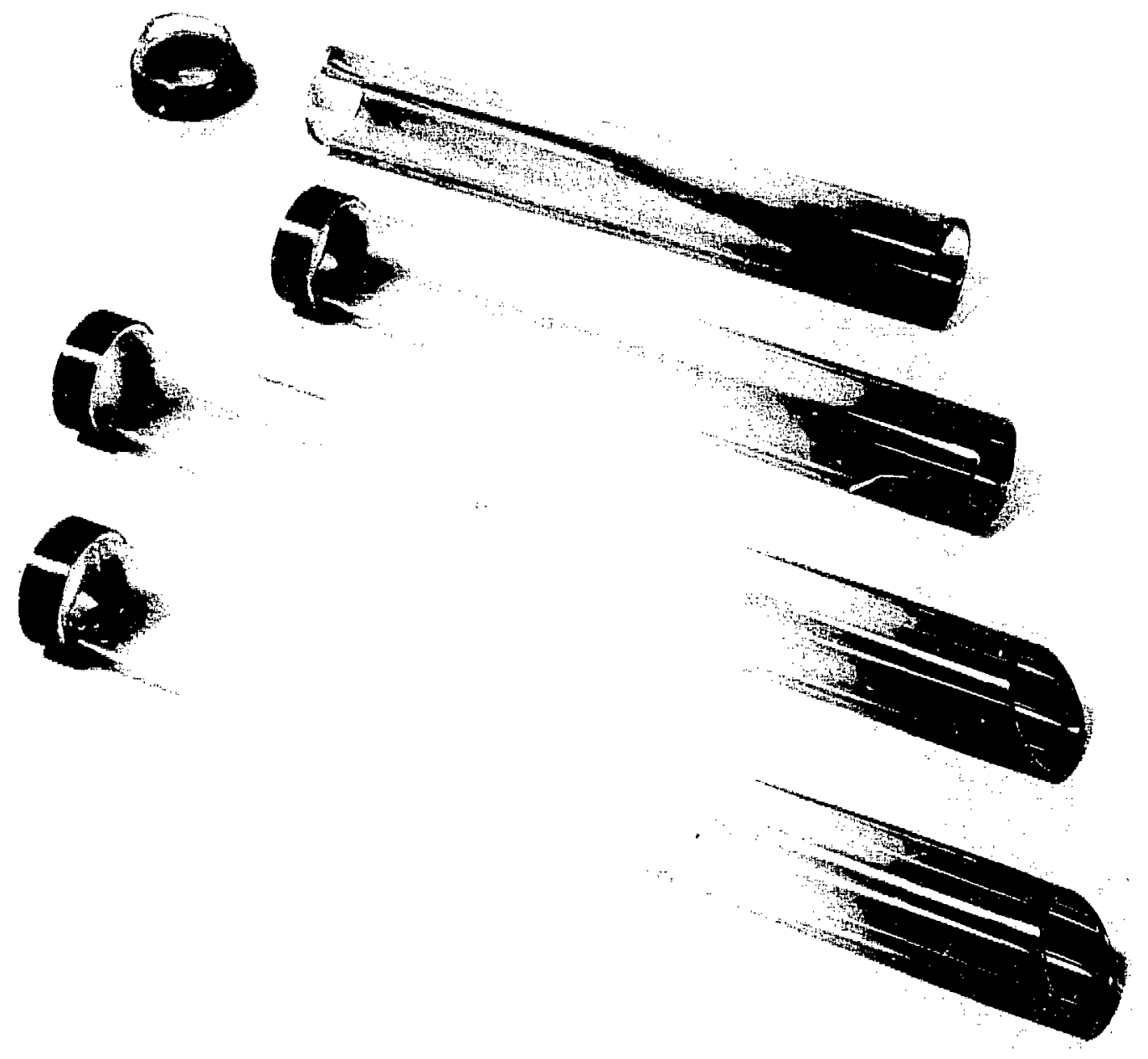

Figure 5.9: Quartz Inserts after Removal from ZT-40M 


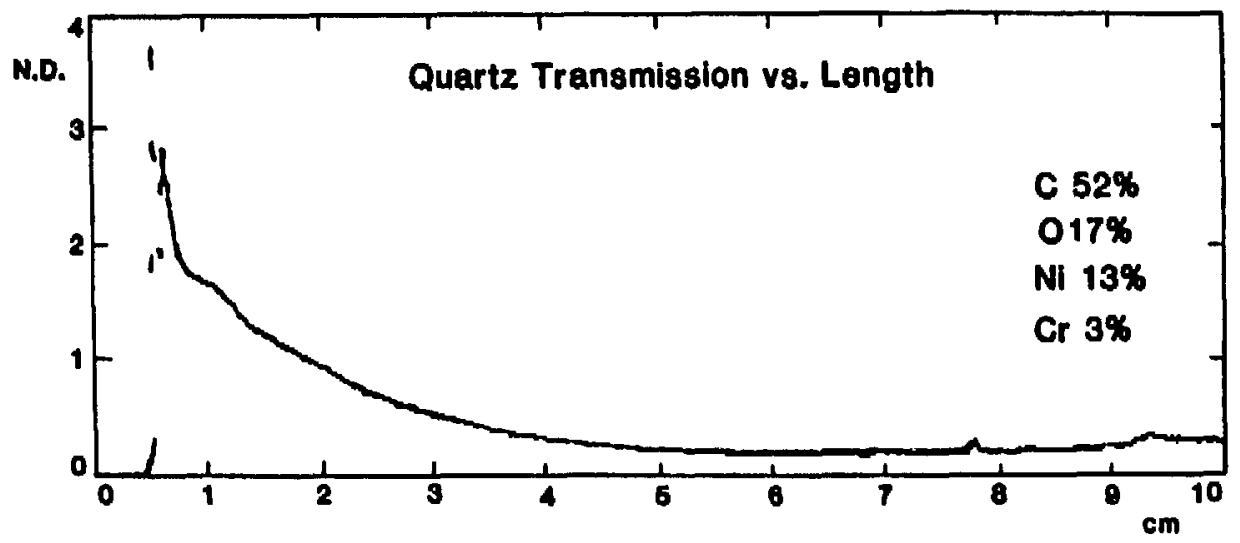

Figure 5.10: Profile of Metallic Deposition on Quartz

siderable coating may have taken place during the pulsed discharge cleaning used routinely on $\mathrm{ZT}-40 \mathrm{M}$ after vacuum openings. Therefore, even before a plasma measurement could be made, the metallic deposits may have destroyed the dielectric properties of the surface exposed to the FIR laser beam.

A sample of the coating was analyzed at Sandia Livermore [103], and was found to consist of $13 \% \mathrm{Ni}, 3 \% \mathrm{Cr}$ (the same ratio as Inconel), $52 \% \mathrm{C}$, and $17 \% \mathrm{O}$. Measurements were made of the deposition profile along the length of the quartz tube. This was done by measuring the attenuation of a visible light source through the side of the quartz tubes. The results are shown in Figure 5.10. The attenuation does not decay to zero because the quartz tube acts as a cylindrical lens when viewed from the side. The measurements indicate that the deposits cause an attenuation of Neutral-Density $\sim 3$ (corresponding to $10^{-3}$ of the incident signal being transmitted). Measurable deposition is seen several diameters $(d \simeq 15 \mathrm{~mm}$ ) along the tubulation, even though the quartz was only in the machine a short time. One of the side benefits of the quartz insert installation was a measurement of metallic deposition, which is relevant to estimating the lifetime of a vacuum window on ZT-40M before attenuation could cause errors in various diagnostic systems. The quartz inserts did not, however, eliminate the polarimetric sensitivity problem. 


\subsubsection{Threaded Inserts on ZT-40M}

It was realized that threaded inserts would not be affected by metallic deposition because they are a geometrical rather than material solution to the vibration sensitivity. Threaded inserts were installed on the central chord tubulations and the $R=R_{o}+8.3 \mathrm{~cm}$ chord tubulations. (The edge chord position was changed because new limiters had been installed in ZT-40M that occluded the $R=R_{o}+12.4 \mathrm{~cm}$ chord.) The effect of the threaded inserts is shown in Figure 5.11. The first two traces appeared earlier (see Figure 5.1). The third trace shows the polarimetric phase with the beam propagated through the torus with threaded inserts during a plasma shot. The plasma is considerably different from the earlier case in the first trace, having a 1 msec pulselength rather than a $10 \mathrm{msec}$ duration. Also, the FIR laser power was lower (and the threaded inserts attenuated the beam somewliat), which caused the higher level of noise after the plasma termination. The anomalous azimuth rotation observed on the first trace, however, is drastically reduced. This allowed useful measurements of Faraday rotation on ZT-40M, as will be described in the next chapter. 


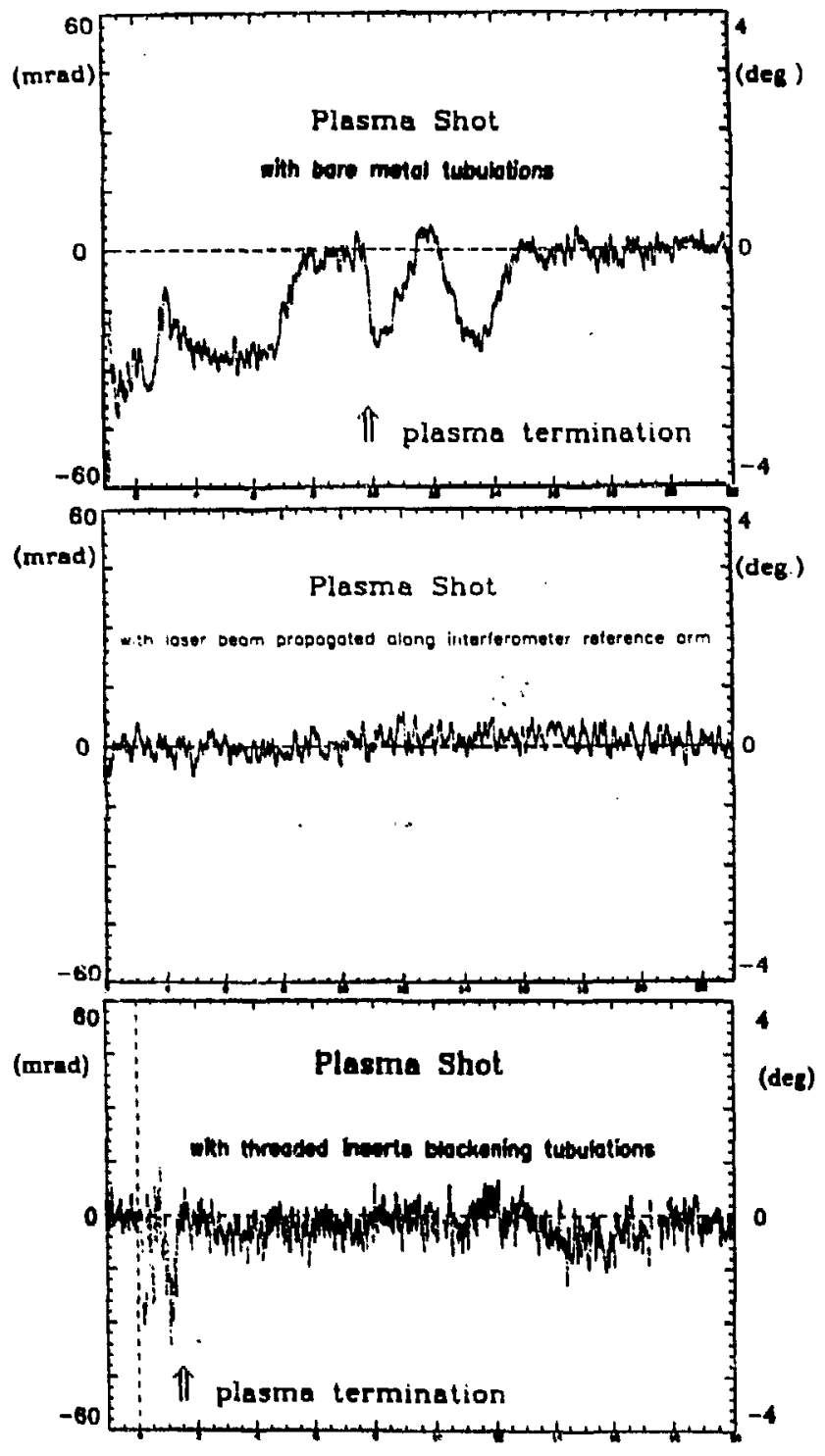

Figure 5.11: Polarimetric Phase for ZT-40M Discharges with Bare Tubulations, Bypassing Torus, and with Threaded Inserts 


\section{Chapter 6}

\section{Measurements on $\mathrm{ZT}-40 \mathrm{M}$}

The development described in Chapter 4 resulted in sufficient interferometric sensitivity, allowing density measurements to be made relatively early in the overall diagnostic development process. Although measurements were only taken on single chords, some interesting information was obtained from the analysis of the measurements in conjunction with othe: measurements on ZT-40M. The mechanical structure that formed the Mach-Zehnder configuration around the machine could be modified to allow the single-chord measurements to be made on different chords. Therefore, some indications of profile effects were obtained, even though detailed profile measurements could not be made because multi-chord measurements were not available.

The far-infrared system operated as an interferometer while the polarimetric development was in progress. Some of the contributions of the initial density measurements are listed below:

- Comparison of the far-infrared interferometer edge-chord measurements with the central chord measurements of another interferometer indicated a flattening of the density profile at higher current operation of ZT-40M.

- Edge-chord density measurements contributed to the determination of the inversion layer radius of sawtooth oscillations observed on ZT-40M.

- The far-infrared interferometer provided back-up measurements on ZT-40M during important data runs, and provided the primary density measurements when other interferometers were not operational. 
Information from the polarimetric measurements was obtained even before the severe sensitivity problems (see Chapter 5 ) were solved. The initial sensitivity was sufficient to resolve the Faraday rotation for a high-current, short-pulsed mode of ZT-40M operation. These measurements showed that the level of Faraday rotation observed was consistent with predictions from theoretical magnetic field profile estimates, coupled with density profile indications from other interferometcrs. After the polarimetric sensitivity problems were solved, Faraday rotation measurements indicated that the spatial profiles of the plasma were quite different for long-pulsed ZT-40M operation, compared to the short-pulsed mode mentioned above. The level of Faraday rotation observed was much lower than expected, indicating that the actual density and/or current profiles were broader than theoretical profile estimates. Also, the profiles became even more broad (or even flat) as the plasma current was increased. The contributions from the current and density profiles could not be distinguished in detail, however, because multi-chord simultaneous measurements of both Faraday rotation and density were not available.

Simultaneous measurements of Faraday rotation and density were made along a single chord on ZT-40M. Although they were made before the polarimetric sensitivity problems were solved, the measurements demonstrated that there was no fundamental problem with the technique. The measurements indicated that the current profile was altered during sawtooth oscillations, in addition to the density profile changes that were indicated earlier. Again, simultaneous measurements on several chords would be required to unfold the density and current profiles in detail. The important extension of the diagnostic to multi-chord operation will be discussed in Chapter 7.

\subsection{Interferometric Measurements}

Interferometric measurements were possible before all of the sensitivity-related development was completed. Much of the sensitivity work was motivated by the initial polarimetric measurements that indicated some serious problems. Large interferometric phase shifts were induced by ZT-40M plasmas, large enough that most of the sensitivity work was not required for interferometric measurements.

The most important developmental step that resulted in sufficient interferometric sensitivity was the elimination of the effects of vibrations and frequency 
drifts of the rotating grating. This was done by installing a reference detector that monitored the signal generated by the grating, and allowed the subtraction of the vibration and frequency drift structure from the interferometric phase. This twosignal technique (discussed in Section 4.2.4), eliminated the problem illustrated in Figure 4.9 , and allowed useful density measurements to be made.

There were other interferometers on ZT-40M, and the role of the FIR interferometer was to provide back-up intensity measurements during down-time on other interferometers and provide supplemental information by measuring edgechord densities when profile information was of interest. The main interferometer on 2T-40M was a two-color $\mathrm{CO}_{2}$ interferometer (see Section 3.3) that measured the central-chord density[104]. The He-Ne laser monitored the vibrations in a Michelson configuration, and a servo mechanism used the vibrational information to maintain a constant cavity length of the interferometer. This allowed the density information to be analyzed quickly. It was the only interferometer that did not require numerical analysis, which could delay results by several minutes because of burdens on the ZT-40M computer system. The other interferometer was an eightchord, single-color $\mathrm{CO}_{2}$ interferometer. This was the only interferometer that could provide profile information; however, mechanical vibrations severely hampered the performance of the interferometer after a few milliseconds. It was useful for studies of plasma formation and measurement of density profiles for very short discharges, but was only useful for fluctuation studies at later times during standard ZT-40M discharges. The role of the FIR interferometer as a back-up density measurement allowed some contributions to be made while the primary task of developing a magnetic field diagnostic was in progress.

\subsubsection{Observation of Refractive-Bending Lffects}

An example of the density measurements that were made with the two-signal technique is shown in Figure 6.1. The measurement was made with the configuration of Figure 4.13. The top trace shows the toroidal current measured by a Rogowski coil. This can be considered to be the toroidal plasma current for the purpose at hand; however, there is a small contribution to the Rogowski coil measurements from currents flowing in the vacuum liner. The second trace shows the carrier signal from the interferometer, and the third trace shows the carrier signal from 

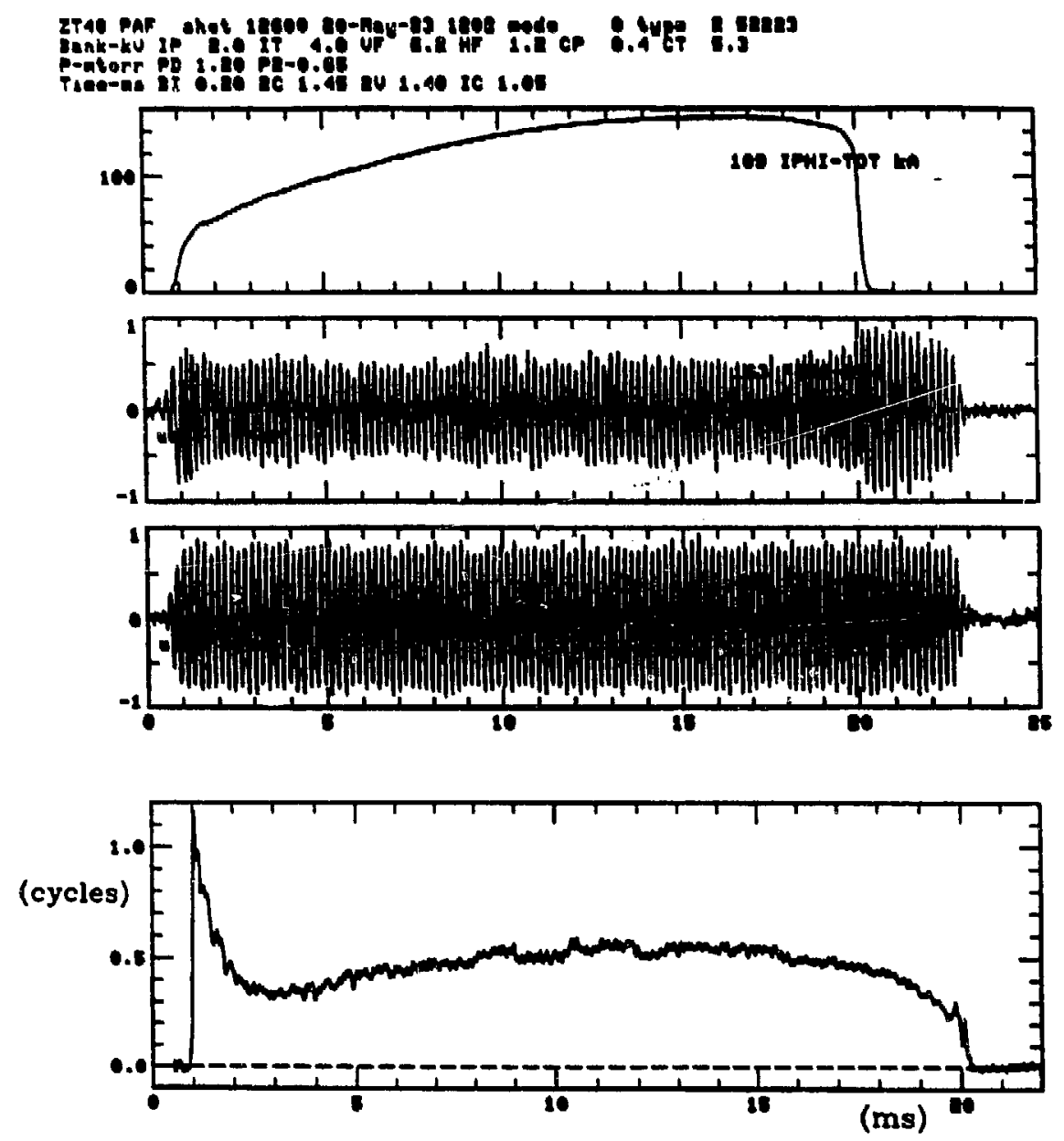

Figure 6.1: Interferometric Measurement with Two-Signal Technique 
the reference detector, measured during the plasma discharge. The carrier signals actually have a frequency of $\sim 40 \mathrm{kHz}$. The apparent slow frequency of the carrier signals is a plotting artifact generated when only a small fraction of the time points are plotted. This "aliasing" occurs when the time interval between plotted points approaches an integral number of periods of the $40 \mathrm{kHz}$ signal. (If the time period between plotted points was chosen carefully, this trace could be made to be a straight line.) Note also that the carrier signals appear in an envelope covering $\sim 22 \mathrm{msec}$. This is the result of the segmented nature of the rotating grating (see Section 4.2.4). The bottom trace shows the calculated phase difference between the two carrier signals, generated by the plasma refractive index. The zero of the phase difference is defined to be zero in the 2 milliseconds after termination. There is no reason, in principle, that this could not be done before the plasma, except that fringe skipping is often observed due to the fast density changes and electrical noise during plasma formation. Fringe skipping refers to the missing of complete cycles of phase shifts (see Section 3.3.1). Fringe skipping is rarely observed at termination, therefore the zero definition after the plasma termination ensures that the phase plot will fall within the expected plotting limits. Even if fringe skipping occurs, there is no lost information. The phase shift is only superimposed on a phase of $2 n \pi$. Since there is only $\sim 1$ fringe of density to measure, there is never any ambiguity of the real phase determination.

Amplitude changes caused by refractive bending can be clearly seen of the carrier signal. The amplitude is slowly decreased to $\sim 60 \%$ of the original level during the plasma formation periods (from 1 to $2 \mathrm{msec}$ ), and is abruptly increased to the original level upon termination of the plasma. This reduction in amplitude may have been caused by attenuation of the scene beam due to increased vignetting of the beam at the exit aperture caused by refractive bending. Alternatively, the reduced carrier signal amplitude could be caused by a reduced overlap of the scene and reference beams of the interferometer, again caused by refractive bending. Both possible causes may have contributed to the effects seen in Figure 6.1. In any case, this is a clear demonstration of the virtues of heterodyne interferometry, where the reduced amplitude of the carrier signal caused by refractive effects (or any other effects) do not directly cause uncertainty in the measurement accuracy.

It was at this point that it was discovered that the main waveguide (which was used to propagate the beam through the 17 meter distance between the laser screen 
room and ZT-40M) elliptized the linear polarizations. This caused extinction ratio errors (see Equation 4.8) that might have caused as much as a $10 \%$ uncertainty in density measurements such as that shown in Figure 6.1. As mentioned in Section 4.3 , the linear polarizations could be restored by introducing a $\lambda / 4$ plate at the waveguide exit. The simultaneous configuration requires an additional $\lambda / 4$ plate to convert the linear polarizations to circular. An equivalent function can be obtained by a single $\lambda / 4$ plate, however, by orienting it such that the elliptical polarizations exiting the waveguide are converted directly to circular polarizations. Therefore, it was decided to solve the polarization problem and set up the interferometer in the configuration that was required for simultaneous measurement at the same time (see Figure 4.14). In addition to solving the extinction ratio problems, an important intermediate step in the development of the simultaneous measurement could be taken. Since interferometric measurements were being made alone, the beamsplitter that allows some signal to go to the polarimeter detector was replaced by a mirror.

It was then that another extinction ratio problem was discovered, this time attributed to the $G a A s$ photoconductive detectors not behaving as ideal squarelaw detectors. As discussed in Section 4.4.3, this problem was solved by filtering the spurious polarization before it was incident on the interferometric detector of the simultaneous configuration. Once this was done, accurate density information was taken rather routinely.

\subsubsection{Time Evolution of Plasma Density on ZT-40M}

An example of interferometric measurements during standard ZT-40M operation is shown in Figure 6.2. By this point in time, the polarimetric sensitivity work had led to the use of a four-signal phase-subtraction technique (see Section 4.2.4), which was also employed for interferometric measurements, even though sensitivity was not a concern for the interferometer. The top trace of Figure 6.2 shows the chord averaged density in units of $10^{13} \mathrm{~cm}^{-3}$. The second trace shows the toroidal current, and the remaining traces show the four carrier signals required to extract the plasma refractive index from other effects that can cause spurious phase shifts. The first carrier signal is from the reference detector during the plasma discharge, and the second is from the interferometer detector during the same time-period (note that refractive bending effects are even more severe). The third and fourth 

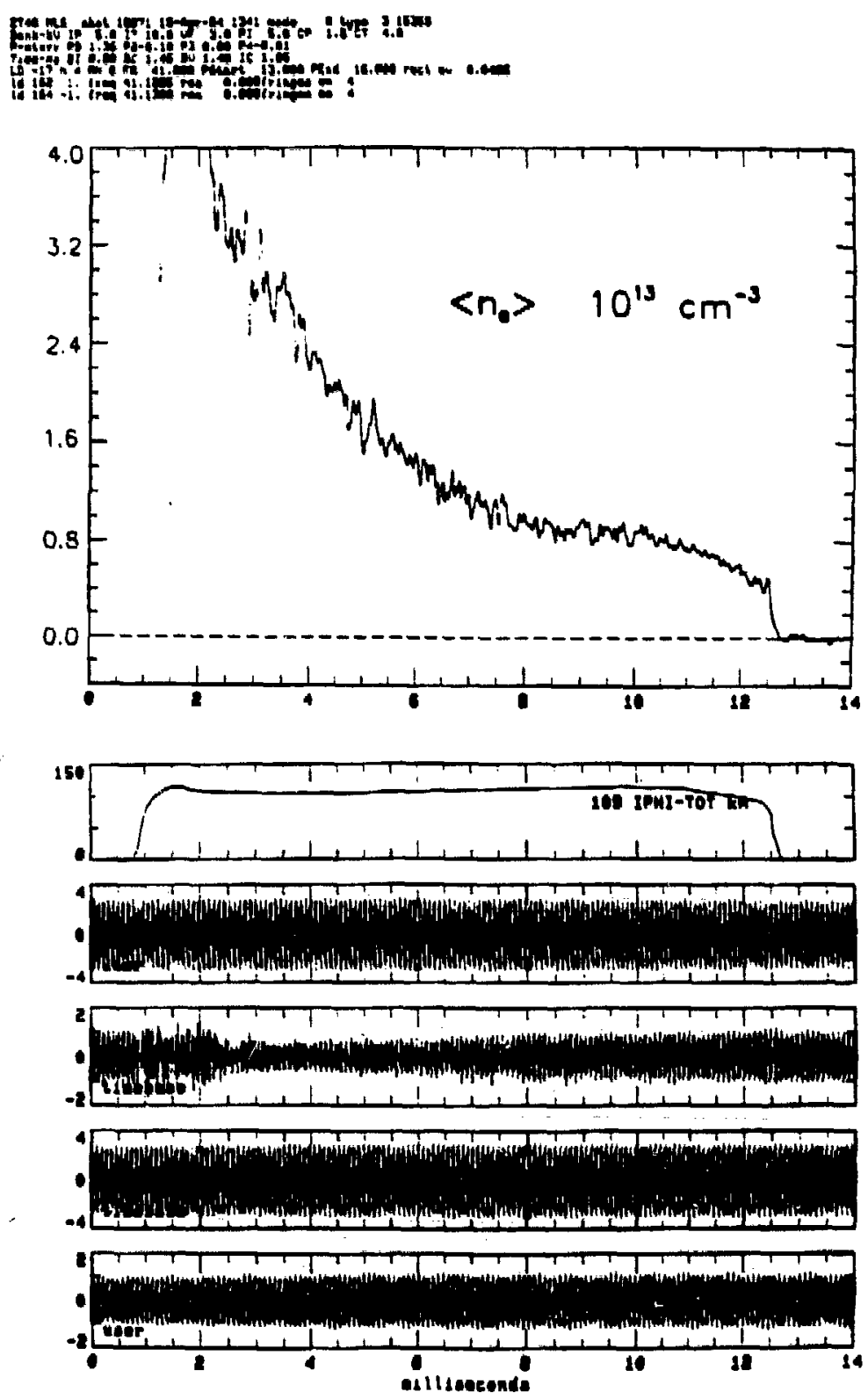

Figure 6.2: Example of Interferometric Measurement in Simultaneous Configuration 
signals are from the reference and interferometer detectors, respectively, taken one grating wheel revolution before the plasma discharge. The top trace, showing the inferred plasma density, illustrates the commonly observed phenomenon of fringe skipping (see Section 3.3.1). There should be a zero density level before the current rise time $(0.75 \mathrm{msec})$, however transients have caused the missing of complete cycles of phase shift. This happened during the formation phase of the discharge, and explains why the zero phase is determined after termination, where fringe skipping rarely occurs. Interferometers that measure the density of tokamak plasmas frequently have similar problems during disruptions, where density changes take place on is fast timescale. By increasing the heterodyne frequency, these fringe skipping problems can be avoided.

For standard operation of $2 \mathrm{~T}-40 \mathrm{M}$, the density time evolution follows a well established and reproducible path. The initial density is quite high, corresponding to a fairly complete ( $\geq 80 \%$ ) ionization of the fill gas. A decay of the density follows (known as density pump-out); the time constant of the decay depends greatly on the conditioning of the vacuum wall, which can be an important source of impurities and reservoir of neutral deuterium. The density reaches an asymptotic level, determined by the balance between particles lost by the plasma and an influx of recycled deuterium. For this particular discharge, the power crow-bar of the toroidal current was turned off at $\sim 10 \mathrm{msec}$, and the plasma "coasted" to $12.5 \mathrm{msec}$, when termination took place.

\subsubsection{Particular Measurements by the Far-Infrared Inter- ferometer}

The far-infrared interferometer provided density measurements routinely on ZT$40 \mathrm{M}$, but there were certain data runs where particular contributions were made. During a data run for investigating the effects of gas puffing and determining the scaling of confinement parameters as a function of current, the far-infrared interferometer indicated that density profiles changed with the plasma current level. Figure 6.3 shows the chord-averaged density from the central chord (measured by the two-color $\mathrm{CO}_{2}$ interferometer) compared to the $R=R_{o}+12.4 \mathrm{~cm}$ chord (measured by the FIR interferometer). Figure 6.3a shows the comparison for $120 \mathrm{kA}$ plasma current, and Figure $6.3 \mathrm{~b}$ shows the comparison for $240 \mathrm{kA}$ discharges. At the lower current, the edge-chord density is lower than the central-chord density, 

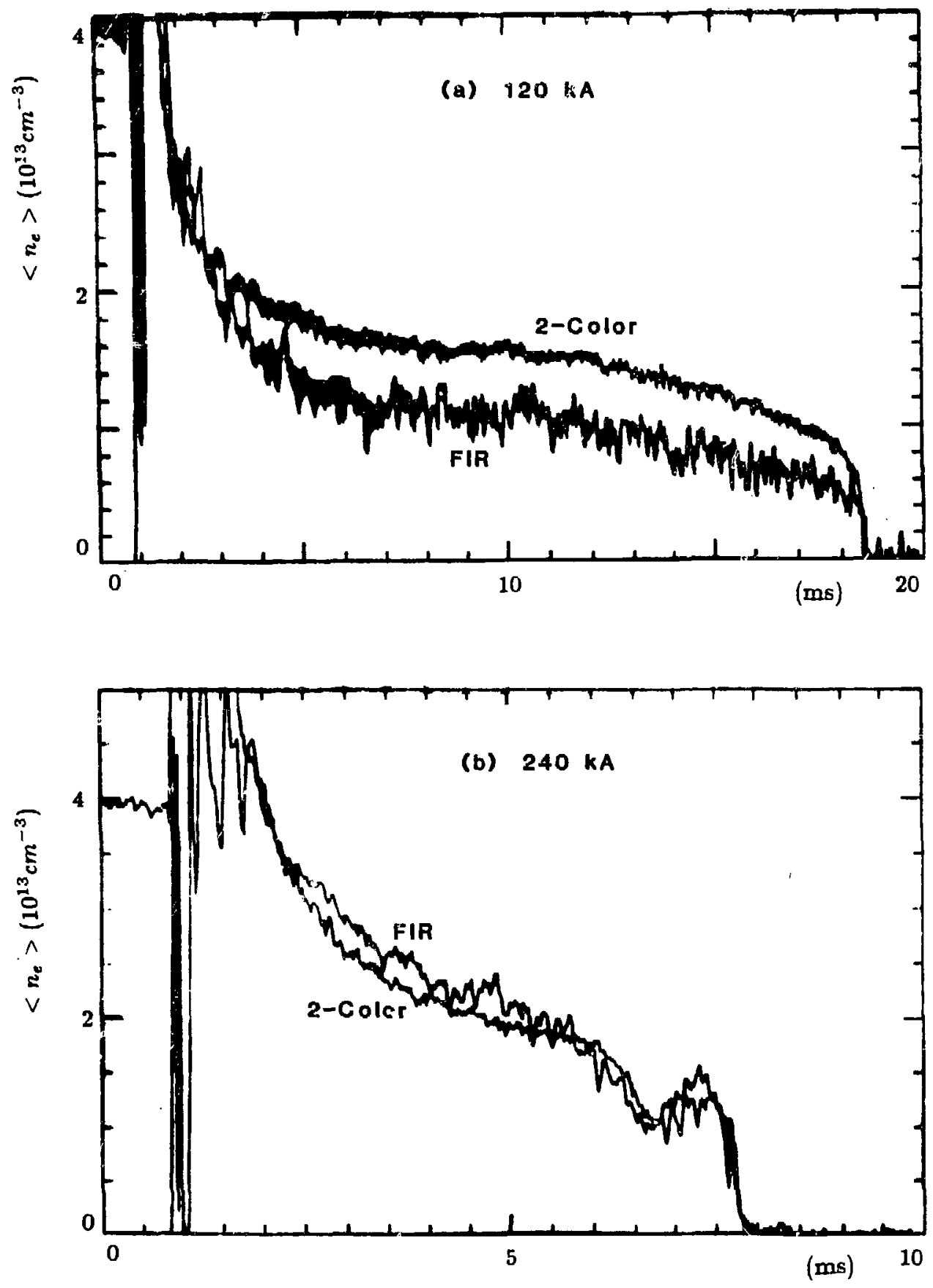

Figure 6.3: Central- and Edge-chord-averaged Densities 
indicating a peaked profile, while in the higher current shots, the edge-chord density is roughly the same as the central-chord density, which would indicate a flat profile. The confinement scaling was found to degrade at the higher current levels, although it is not clear whether the profile changes were a symptom or a cause. The introduction of gas puffing caused the profile at $120 \mathrm{kA}$ to become flat and the $240 k A$ profile to become slightly hollow.

During a data run when the understanding of sawtooth oscillations was being pursued, the far-infrared interferometer helped in the determination of the inversion radius of sawtooth oscillations. Sawtooth osciliations are characterized by a peaking of the density and temperature profiles followed by a sudden internal "crash" of these profiles that causes them to flatten. This process repeats on millisecond timescales. One would expect the central measurements of temperature and density to increase during the profile peaking phase, and fall sharply as the profiles flatten. Edge diagnostics should see an inverted sawtooth, where the density or temperature slowly decrease as the profiles peak and suddenly rise when the central density and temperature "crash". An important parameter in the study of sawteeth is the "inversion" radius - the transition between the central and edge sawtooth signatures.

Sawteeth are usually most clearly observed in soft $x$-ray diagnostics which are sel.sitive to density and temperature (by detecting bremsstrahlung radiation, which is $\propto\left(z^{2} n^{2} / \sqrt{T}\right) e^{-h \nu / T}$ ) as well as impurity line radiation (which can confuse the sawtooth measurement). The $\mathrm{x}$-ray measurer.ents are centrally weighted, where the density and temperature values are highest. An interferometric density measurement is uniformly weighted, and not confused by impurity line radiation, and hence can help resolve the inversion radius. Figure 6.4a shows the sawtooth oscillations on the density that are right-side-up, indicating that the inversion layer is beyond the $12.4 \mathrm{~cm}$ chordal position. Figure $6.4 \mathrm{~b}$ shows the oscillations for another shot, where the sawteeth are no longer well defined, indicating that the chordal path through the plasma is sampling portions outside the inversion radius as well as within the inversion radius. If the sampled portions are assumed to be of equal lenght along the chord, the inversion radius is determined to be at $r=15 \mathrm{~cm}$.

The far-infrared interferometer often functioned to back up other interferome- 


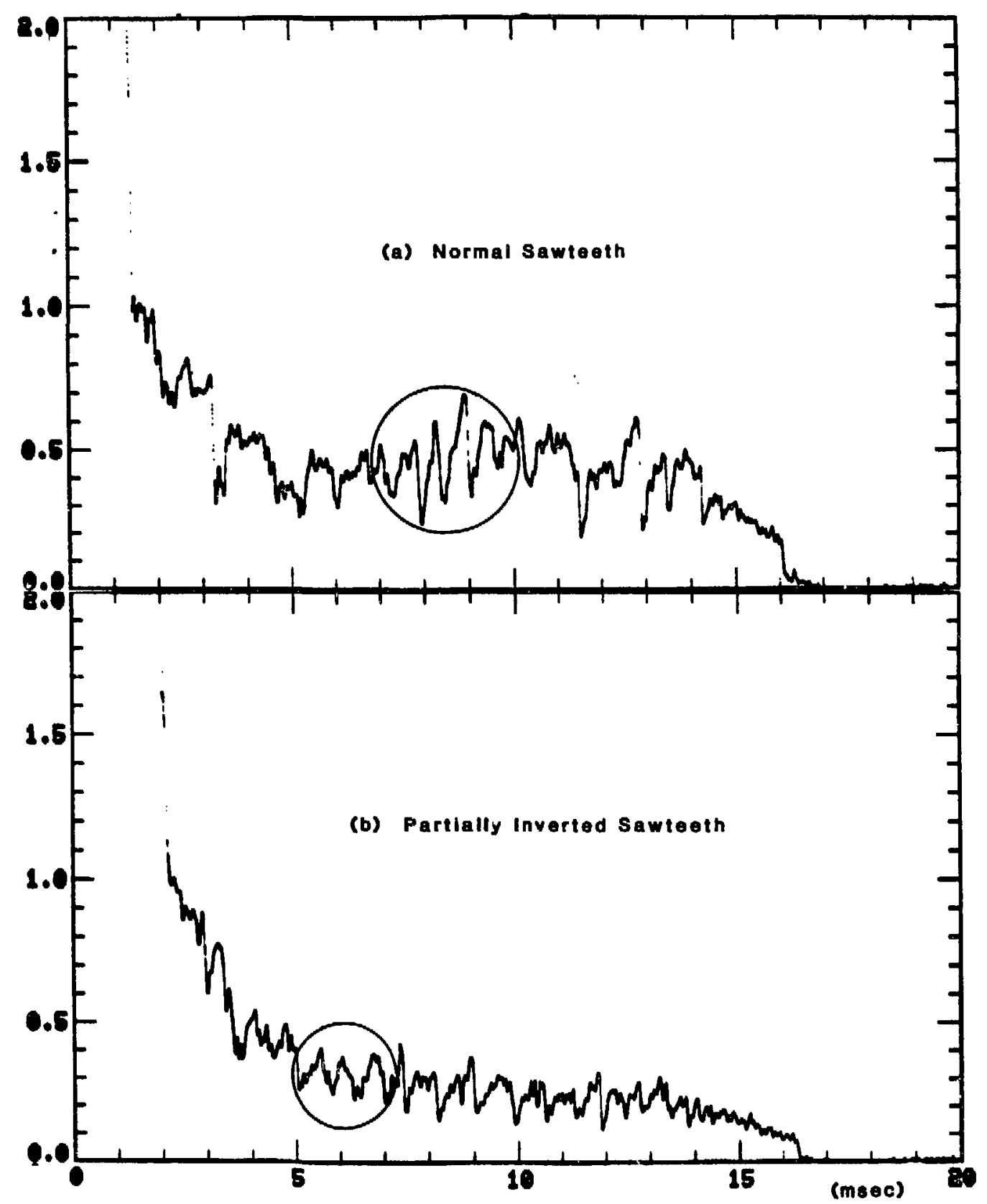

Figure 6.4: Examples of Density Sawtooth Oscillations 


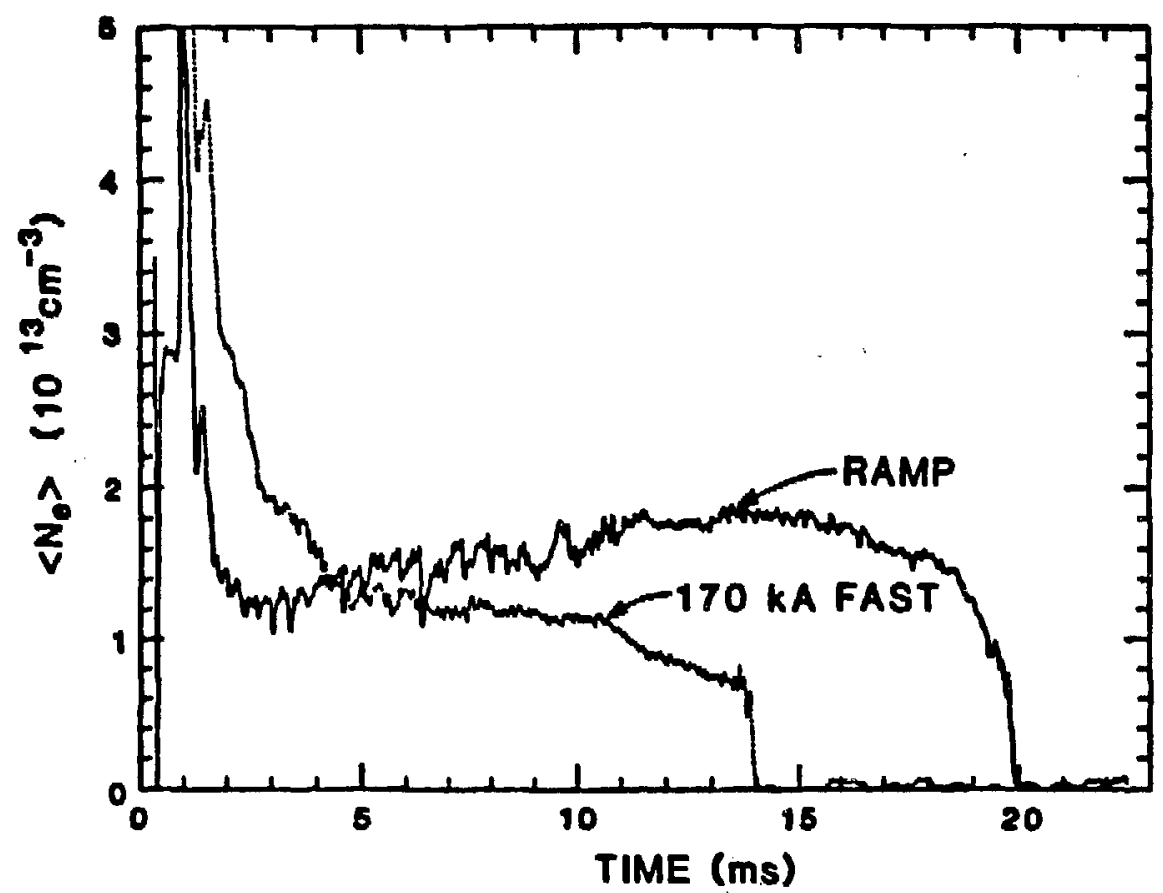

Figure 6.5: Density of Ramped (70 $k A$ to $170 k A$ ) Discharge Compared to Standard $170 k A$ Discharge

ters. This was of particular importance during a current ramping run, where the plasma was formed at a relatively low plasma current $\left(I_{\phi}=70 \mathrm{kA}\right)$ and the power crow-bar was used to drive the current level higher $\left(I_{\phi}=170 k A\right)$ [105]. (Figure 6.1 is an example of such a discharge.) This procedure has important impacts on start-up voltage and power requirements for the next generation RFP devices as well as RFP reactors.

The central-chord density, measured by the FIR interferometer during a ramped discharge, is plotted in Figure 6.5 along with density measured by the two-color interferometer during a standard 2T-40M discharge. The standard discharge had a plasma current level corresponding to the final current of the ramped discharge. The final density of the ramped discharge actually exceeded the density corresponding to the same current level of the standard discharge. This would suggest that the plasma density depends not only on the current level, but the time history of the plasma formation and evolution. The higher chord-averaged density could also result from profiles being more peaked in the ramped discharge. It could be that 


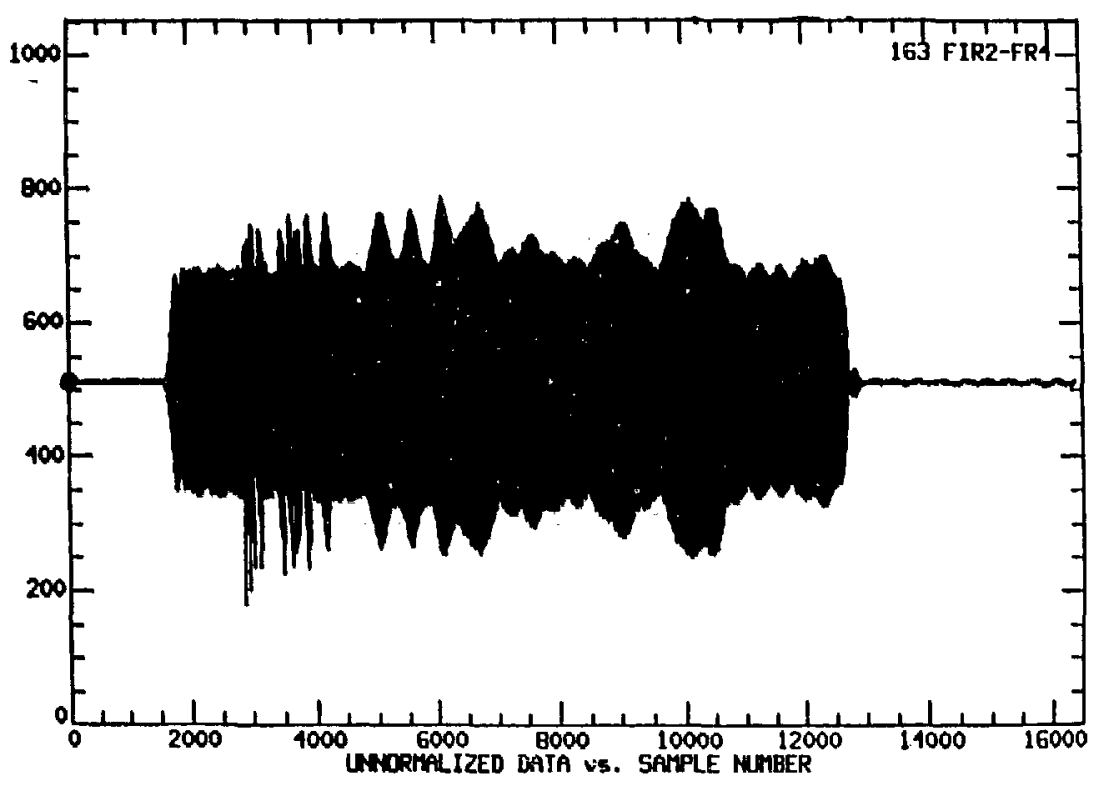

Figure 6.6: Carrier Signal Measured by the Polarimeter

the nominal low-current profiles (known to be more peaked) are retained in the ramped discharge up to higher currents. Clearly, the detailed distinction of contributions from profile effects requires multi-chord density measurements in order to be able to invert the spatial profiles.

The role of the far-infrared interferometer as a back-up or supplemental interferometer allowed contributions to the understanding of confinement physics to be made while the primary task of developing polarimetric measurements was being pursued. The measurement of Faraday rotation by the polarimeter will be presented in the following section.

\subsection{Polarimetric Measurements}

The first polarimetric measurement that should be shown is simply a trace of the carrier signal measured by the polarimeter detector in the configuration of Figure 4.12. The carrier signal appears in Figure 6.6, and very strong amplitude variations can be seen. The vibrating diagnostic tubulations may contribute to the amplitude changes; however, the important observation is that such amplitude 
changes preclude the use of amplitude polarimetry on ZT-40M. To measure Faraday rotation, heterodyne polarimetry must be employed.

There were more serious problems in obtaining sufficient sensitivity of the polarimeter, and although the development that was required for the interferometer also improved the polarimetric sensitivity, further developments described in Chapter 5 were required to allow Faraday rotation measurements on general ZT-40M discharges. There was a special class of ZT-40M discharges, however, that were not prone to the tubulation vibration effects and allowed Faraday rotation measurements before the developments of Chapter 5 were implemented.

This special mode of operation was motivated by wall damage problems that were observed in moderately high-current, sustained discharges. Vacuum leaks in the inconel bellows were generated by excessive heating and stresses caused by plasma-wall interaction. This limited the plasma current levels that could be safely obtained with the standard discharges in ZT-40M. Higher current data was of primary interest, however, because a determination of how confinement parameters scale with current is very important in the prediction of the performance of next generation machines, and the ultimate performance of RFP reactors. The curzent levels safely obtained in standard ZT-40M discharges did not cover enough range to make confident predictions of the scaling. By shortening the discharge time from $10-20 \mathrm{msec}$ to $1-2 \mathrm{msec}$, higher current levels could be obtained without a much higher vacuum liner heating than that produced in lower-current sustained discharges, because the short duration reduced the total energy incident on the vacuum liner. The mode of operation was called "design-mode" because the timescale, ironically, was similar to the original design specifications of ZT-40 (see Section 4.1).

An example of the Faraday rotation measured during a design-mode discharge is shown in Figure 6.7. The top trace shows the toroidal current. Density measured by the eight-chord $\mathrm{CO}_{2}$ interferometer indicated that the plasma terminated at $\sim 2.4 \mathrm{msec}$, which would suggest that the toroidal current beyond the kink in the current waveform (at $\sim 2.4 \mathrm{msec}$ ) was actually flowing in the vacuum liner. The second trace shows the Faraday rotation at the $R=R_{o}+12.4 \mathrm{~cm}$ chord. The reason that the Faraday rotation stands out above the vibration-induced structure discussed in Chapter 5 was because the anomalous structure occurred after termination of these short $d$ iharges. 


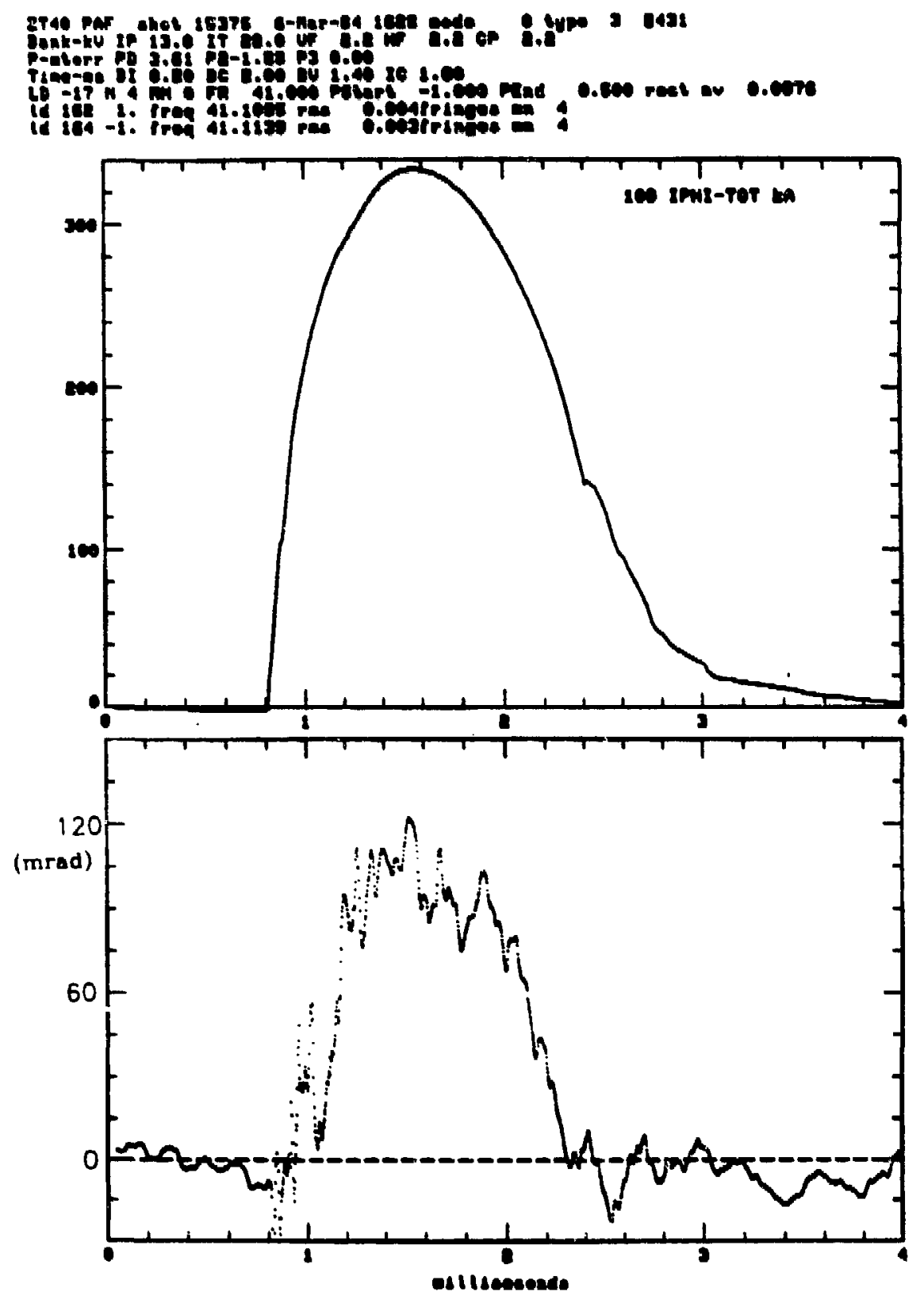

Figure 6.7: Toroidal Current and Faraday Rotation for a Design-Mode Discharge 
The expected Faraday rotation for this discharge, assuming a parabolic density profile (which was indicated by measurements of the eight-chord $\mathrm{CO}_{3}$ interferometer) and using

$$
B_{p}(r)=\frac{\mu_{o} I}{2 \pi a J_{1}(15 a)} J_{1}(15 r),
$$

is $\sim 100$ mrad at peak current. This is in good agreement with the peak Faraday rotation observed, and therefore the measurement was consistent with a first-order Bessel function poloidal field profile, although by no means uniquely specifying that profile.

There is also an apparent lag between the current rise and Faraday rotation evolut in. This is consistent with the plasma forming as an annular sheath at a large minor radius and diffusing inward in a time scale of hundreds of microseconds. It was also observed on various classes of discharges that for a low fill pressure, there was a negative Faraday rotation during the formation phase of the discharge that was not observed on high fill pressure discharges (the discharge of Figure 6.7 had a fill pressure of 3.61 mtorr, which was relatively high). This seems to indicate that the breakdown position was changing as a function of fill pressure.

Once the polarimetric sensitivity problems were solved, Faraday rotation measurements were made on more general classes of ZT-40M discharges. An example of Faraday rotation measurement appears in Figure 6.8. Each trace in the figure shows the average (solid lines) \pm the standard deviation (dashed lines) for 10 discharges at $300 \mathrm{kA}$. The density was measured by the two-color interferometer. The vibrations were severe enough that the compensation ran out of dynamic range, which caused problems in the density measurement beyond $6 \mathrm{msec}$.

Had the simultaneous FIR polarimetry/interferometry measurement been made on these discharges, the two-color density would not have been required. The FIR density measurements also would not be prone to the vibration problems, because the longer wavelength operation of the FIR interferometer makes it less sensitive to vibrations by the square of the respective wavelengths ratio, a factor of $\sim 300$. Note that much of the variation in Faraday rotation level resulted from variations in the plasma density, even though attempts were made to produce nominally identical discharges.

Again, the Faraday rotation level increased more slowly than the toroidal current, indicating annular formation and inward diffusion of the current. In stark 


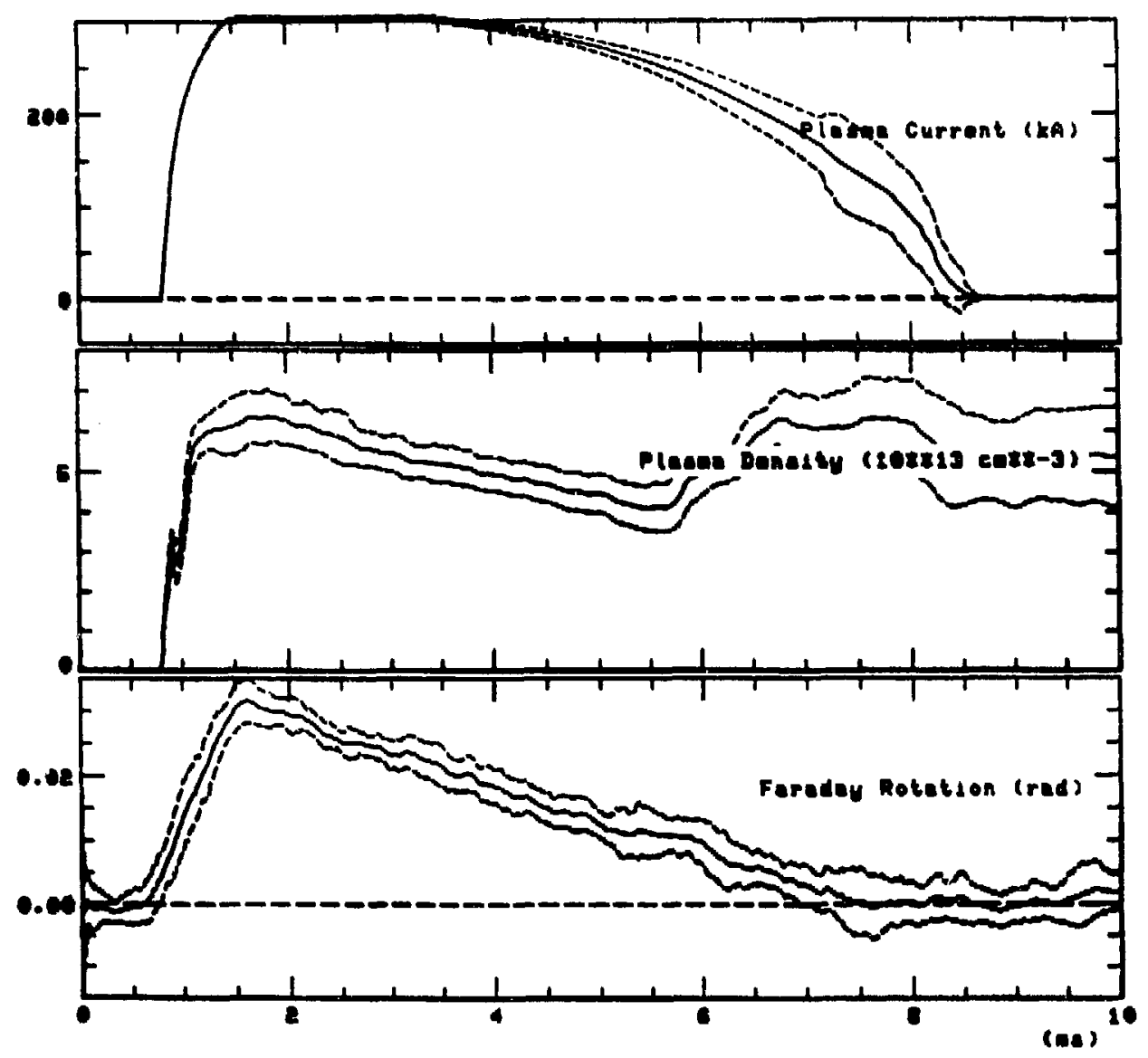

Figure 6.8: Current, Density, and Faraday Rotation for $300 k A$ Discharges 


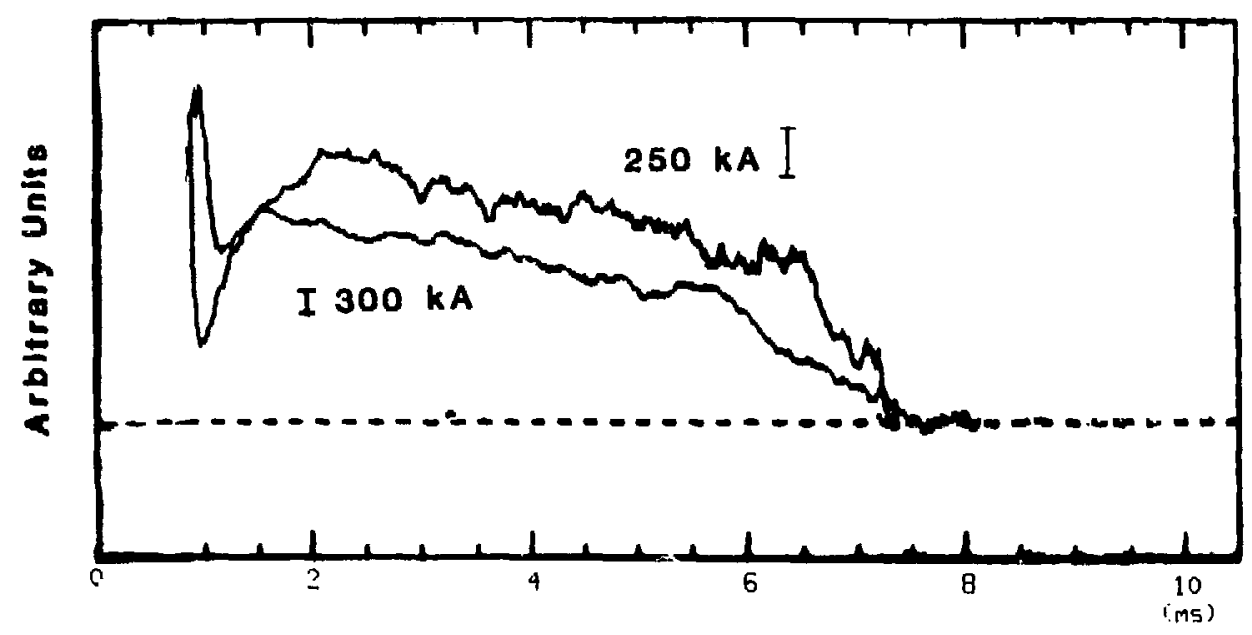

Figure 6.9: Faraday Rotation Normalized by Density and $I_{\phi}$

contrast to the Faraday rotation measured during design-mode discharge, however, the level of Faraday rotation for these discharges is much lower than predictions from theoretical profiles. The same calculations described for the predictions of design-mode Faraday rotation indicated that the expected Faraday rotation for these standard discharges is $\sim 60 \mathrm{mrad}$, nearly twice the observed rotation level. This indicates that the density and/or current profiles are much broader than predicted.

Similar measurements at lower currents indicate that the profiles are not as broad at lower current. Figure 6.9 shows the average Faraday rotation for the $300 k A$ shots, compared to the $250 k A$ shots. The Faraday « tation is normalized by the toroidal plasma current and the central-chord-averaged density. Any variation in this normalized rotation can only be attributed to profile changes. It is clear that the $300 \mathrm{kA}$ normalized rotation is lower than the $250 \mathrm{kA}$ normalized rotation, indicating that the profiles are indeed broadening at higher currents. Although the error bars are larger for lower current data, the trend appears to extend down to the lower currents. With multichord simultaneous measurements of density and 
Faraday rotation, these profile effects could be documented in detail.

Profile effects are an important factor in understanding the scaling properties on ZT-40M. The design-mode discharges exhibited favorable scaling with plasma current, with $\left\langle n_{e}>T_{e o} \propto I^{2}\right.$ for $120 k A \leq I_{\phi} \leq 440 k A$, thus indicating that the plasma $\beta$ was independent of current. For sustained ZT-40M discharges, it was also observed that $\beta$ was approximately constant for $60 k A \leq I_{\phi} \leq 120 k A$. At higher plasma currents (up to $240 \mathrm{kA}$ ), this favorable scaling of density and temperature deteriorated. It was postulated that this deterioration results from increased plasma wall interactions at the higher currents due to uncorrected magnetic field errors at ports and gaps in the conducting shell[8].

The Faraday rotation measurements have indicated that the profiles flatten for the high-current sustained discharges, whereas the profiles for the high-current design-mode conditions did not become flat. It is not clear if there is a causal relationship between the flatter profiles and the scaling results for sustained discharges. There is an indication that the current scaling is not easily determined, because the current level cannot be simply increased without some other changes taking place.

\subsection{Simultaneous Polarimetry and Interferome- try}

The ability to measure the Faraday rotation and density simultaneously is of primary interest for internal field measurements. To infer the magnetic field profile in detail requires an independent knowledge of the density profile. Although it is possible to have a multichord polarimeter that uses data frcm a multichord interferometer at a different location to unfold the profiles, uncertainties can result if there are toroidal variations in the plasma (more likely for an RFP plasma than a tokamak plasma). Also, some of the interesting information that may be obtained from Faraday rotation measurements will come from fluctuation analysis, when correlations between interferometric and polarimetric fluctuations are studied to provide information on magnetic fluctuations. In this case, it is very important that the measurements be made at the same location, because of confusion that would be caused by amplitude evolutions and time shifts as the fluctuations propagate between toroidal locations. There is also a space problem when there are 
more diagnostics than access ports, and the consolidation of the interferometry and polarimetry to one location would free up ports for other measurements.

The demonstration of a simultaneous polarimeter/interferometer was actively pursued before the polarimetric sensitivity problems were completely understood. Even without acceptable polarimetric sensitivity, the simultaneous configuration could be implemented to determine if there were fundamental or unforeseen problems in the diagnostic technique. The first step in the demonstration of the simultaneous configuration was measuring density in the simultaneous configuration. As described earlier, it was found that the detectors did not behave as ideal squarelaw detectors. The intermediate step of making density measurements with the simultaneous configuration revealed that modifications to the optical configuration were required to filter out the unwanted polarization.

The next step involved installing the non-polarizing beam splitter, which was required to allow the polarimetric measurements to also be made. Although lack of enough detectors prevented a true simultaneous measurement, the configuration was assembled to allow both interferometric and polarimetric measurements to be made on successive shots by moving the detector, with no additional change in optics. It was then that problems with the non-polarizing beam splitters were found. It had been surmised that there might be no such thing as an ideal non-polarizi:? beam splitter, because some polarization effects can be caused by slight differences in the $s$ and $p$ reflectivities at an incident angle of $45^{\circ}$. This effect is discussed in detail in Appendix C, where the non-polarizing beam splitters that were used are described, and techniques to minimize the polarizing effects are discussed. Slightly polarizing beam splitters can cause extinction-ratio problems, resulting in errors in the interferometric measurements (see Equation 4.8). With the polarization effects of the "non-polarizing" beam splitter minimized, the degradation of the extinction-ratios was virtually eliminated.

Subsequently, another $G a A s$ detector was mounter in the cryostat, which allowed a true simultaneous measurement to be made. An example of a simultaneous measurement appears in Figure 6.10. The measurement was made on the $R=R_{0}+12.4 \mathrm{~cm}$ chord, at a plasma current of $120 \mathrm{kA}$. The top trace shows the polarimetric phase (calculated with the four-signal phase-subtraction technique), and the bottom trace shows the interferometric phase (also calculated with the four-signal technique). Recall that the polarimetric sensitivity problems were not 


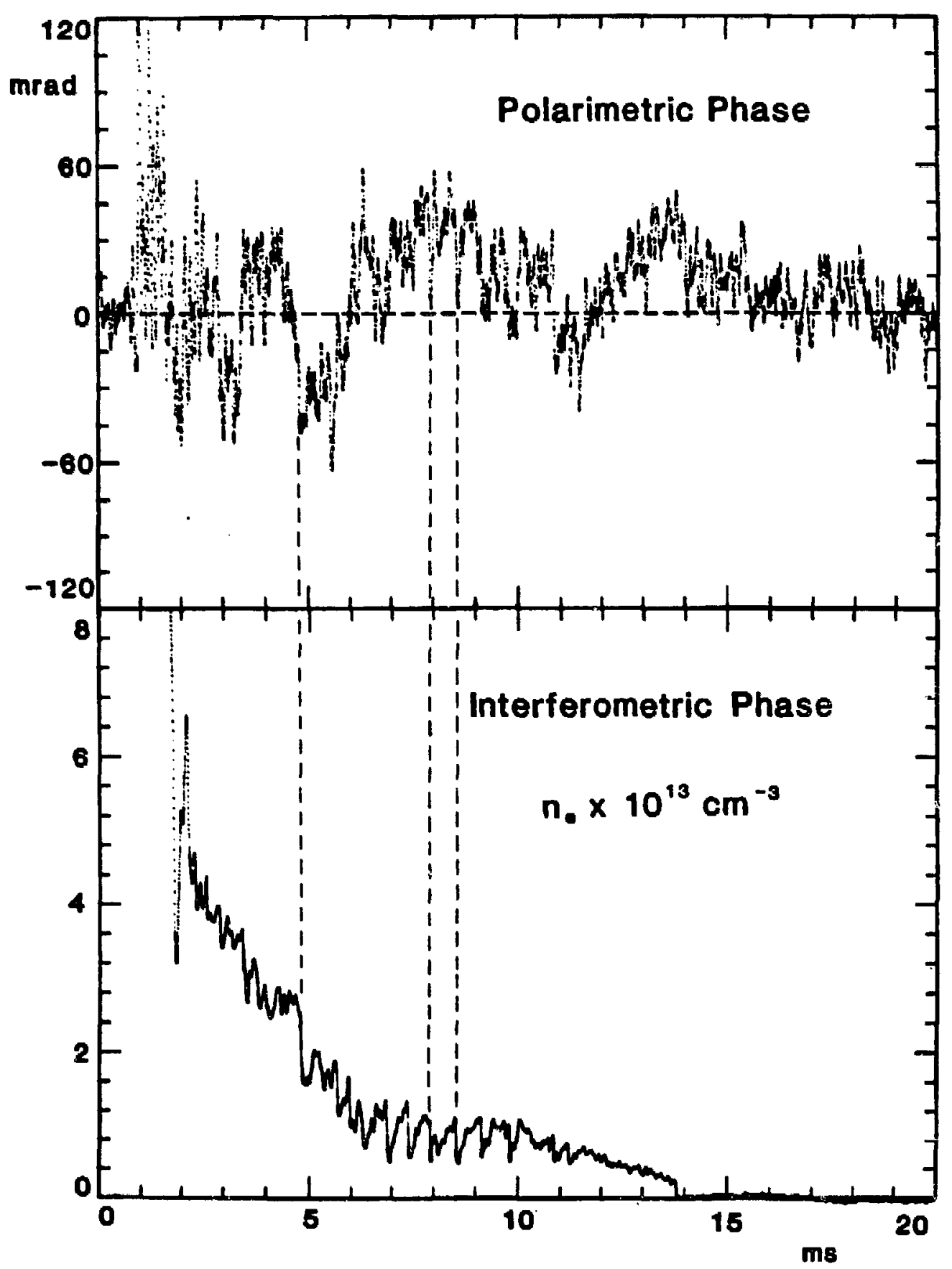

Figure 6.10: Simultaneous Measurement of Density and Faraday Rotation 


\begin{tabular}{ccc}
\hline Current Profile & Density Profile & Rotation $\left(R=R_{o}+12.4 \mathrm{~cm}\right)$ \\
\hline Flat & Flat & $4.2 \mathrm{mrad}$ \\
Flat & Parabolic & $3.4 \mathrm{mrad}$ \\
Equilibrium & Flat & $8.9 \mathrm{mrad}$ \\
Equilibrium & Parabolic & $8.2 \mathrm{mrad}$ \\
Peaked & Flat & $15.6 \mathrm{mrad}$ \\
Peuhed & Parabolic & $14.2 \mathrm{mrad}$ \\
\hline
\end{tabular}

Table 6.1: Calculated Faraday Rotation Levels for Various Profiles

yet solved when this measurement was made, which explains the unphysical structure on the polarimetric phase.

This particular shot is presented because the sawtooth oscillations observed clearly on the interferometric phase also appear on the polarimetric phase. Therefore, even though the sensitivity problems mask the Faraday rotation measurement required to make profile inferences, the sawtooth oscillations allow some analysis of current profile changes from the polarimetric data. The large density drop at 5 msec appears also on the polarimetric phase, and some density sawteeth between 8 and $9 \mathrm{msec}$ are (barely) visible on the polarimetric phase. Although the polarimetric phase trace is quite noisy, all of these features are characterized by phase changes that are larger than can be explained by the density changes alone. The sawtooth amplitude can only be explained if the current density peaks significantly during the sawtonth rise and flattens during the sawtooth crash.

The expected Faraday rotation for the $R=R_{0}+12.4 \mathrm{~cm}$ chord, assuming $n_{\mathrm{c}}=n_{\mathrm{co}}\left(1-r^{2} / \mathrm{a}^{2}\right)$ and $B_{p}(r) \propto J_{1}(\alpha r)$, is only $\sim 8 \mathrm{mrad}$, yet the sawtooth oscillations have $\sim 15$ mrad amplitudes. Table 6.1 shows the calculated Faraday rotation level at the measurement position, assuming either a parabolic or flat density profile for each of three current density profile cases. The "equilibrium" case was calculated by assuming $B_{p}(r) \propto J_{1}(\alpha r)$, which is roughly what is anticipated for an equilibrium RFP plasma. The "peaked" case was calculated by assuming that all of the toroidal current was located within the $12.4 \mathrm{~cm}$ measurement location. The "flat" case represents a flat current density profile. Note that density profile changes have very little effect on the Faraday rotation level, particularly at $12.4 \mathrm{~cm}$. Note also that significant peaking of the current density is required to explain the evolution of the Faraday rotation during the sawtooth oscillation. This implies that 
the current channel contracts dramatically during the sawtooth rise and broadens during the sawtooth crash.

The current peaking during the sawtooth rise can be explained by the evolution of the temperature profile during the sawtooth oscillation. During the sawtooth rise, the temperature profile is known to become more peaked[106]. Since this lowers the resistivity on axis, the current density profile will also become more peaked, causing a large increase in the Faraday rotation level. When the temperature profile flattens during the sawtooth crash, the resistivity on axis increases, causing the current density profile to flatten as well.

Although measurements were made on only one chord, the sawtooth effects were strong enough that statements about the current density profile can be made. These observations of sawteeth in the polarimeter, and the implications on the current density profile agree with a theoretical sawtooth study reported by Werley, et al.|107], where calculations predicted that the current density should become peaked to a point where most of the current is within the $12.4 \mathrm{~cm}$ measurement location.

Figure 6.10 also illustrates another very important point. Aside from the tuoulation birefringence effects on the polarimetric phase, there is a higher level of "baseline" noise on the trace over that observed in the polarimetric measurements alone. This was caused by the lower level of signal available for the polarimetric detector when the density was measured simultaneously. This baseline noise is caused by noise on the carrier signal. As less laser power is incident on the detector, the signal to noise of the carrier beccmes lower, causing a higher level of baseline noise on the phase. Clearly, for a multichord simultaneous measurement of Faraday rotation and density, very significant improvements in laser power at the machine are required. For this reason, a simultaneous measurement $-f_{\text {ter }}$ the polarimetric sensitivity problems were solved was not repeated, because laser power improvements were considered to be mich more important, and efforts turned to implementing such inprovements. Techniques for improving the laser power at the machine will be a major topic of the following chapter. 


\section{Chapter 7}

\section{Summary, Conclusions, and Recommended Future Work}

\subsection{Summary}

The development of an accurate and routine irternal magnetic field diagnostic is perhaps one of the final frontiers in plasma diagnostics for fusion applications. There is little question that information obtained by such measurements would be very useful in advancing the understanding of confinement issues. The interest in magnetic fisld measurement in tokamak plasmas is motivated by a lack of knowledge of the toroidal current distribution, which plays an important role in tokamak stability. In a reversed-field pinch, there is as much uncertainty in the toroidal magnetic field profile as in the poloidal magnetic field profile, and both profiles are intertwined in RFP equilibrium and stability. The real interest in the RFP is a measurement of both field profiles.

sin ideal magnetic field diagnostic would have the following characteristics:

- the ability to provide accurate and unambiguous single-shot magnetic field profiles,

- applicability to general plasma configurations, both present laboratory plasmas and reactor-grade plasmas of the future,

- the ability to make measurements with diagnostic access that is available on most machines, or at least not requiring access that may violate the integrity or hamper the performance of the machine, and 
- simple enough that the magnetic field measurements are reliable and routine.

An analysis of the various proposed measurement techniques reveals that there is no measurement method that has all of these characteristics. A:i of the possible techniques fall short in one or more of these areas. Many of the techniques have fundamental problems in some of these areas, making it unlikely that they will be developed further.

Measurement of the internal magnetic field by Faraday rotation is one of the few options that does not have a fundamental problem in meeting the above requirements. It does have some disadvantage in complexity and uncertainties in the profile determination because of the requirement for inversion of chord-averaged data. It is, however, applicable to any confinement scheme (with the appropriate choice of wavelength). Although there is some difficulty in measuring presentday laboratory plasmas (due to the smali amount of Faraday rotation at a wavelength that is appropriate), Faraday rotation measurements will become easier as reactor conditions are approached. Faraday rotation measurements for poloidal magnetic field determination uses diagnostic access that is routinely available for multichord diagnostics, and is closely related to interferometric techniques that are well-established and almost universally employed. In all, there is clear motivation for the development of Faraday rotation measurements as an internal magnetic field diagnostic.

Considerable effort has been devoted to the developrnent of the capability of making Faraday rotation measurements. The most impressive system to date is the polarimeter/interferometer that has been implemented on the TEXTOR tokamak [40]. The system has provided information on the current deinsity profile, and is used as a plasma position monitor [44]. Other polarimeter/interferometers have been assembled and demonstrated on ISX-B [43] and TEXT [47]. These systems have made multichord Faraday rotation and density measurements, although they have not produc d data as routinely as the system on TEXTOR. The multichannel interferometer on TFTR is being extended to make Faraday rotation measurements with a technique that is very similar to that of the TEXTOR system [45].

One of the major drawbacks of the polarimetric techniques that are used in these systems is sensitivity to spurious changes in signal amplitude. Each of these methods (see Figures 3.4 and 3.5) measures the Faraday rotation by sensing the 
amplitude of the component of the final polarization that is orthogonal to the input polarizatios. As a result, amplitude changes that are not due to the Faraday rotation (such as refractive bending in the plasma) cause direct errors in the Faraday rotation measurement.

This fundamental problem can be avoided by the use of heterodyne polarimetry techniques, in which the Faraday rotation produces changes in the time signature of the detected signal. Heterodyne polarimetry involves the use of a probe beam that has a rotating linear polarization. With no plasma, the signal transmitted through a polarization analyzer is sinusoidal (the polarization orientation varies relative to the analyzer). The plasma-induced Faraday rotation will generate a phase-shift of the sinusoidal signal. The data reduction is identical to the analysis required for heterodyne interferometry, in which the plasma refractive index causes phase shifts in a carrier signal. The time resolution that can be obtained with heterodyne polarimetry is comparable to that of heterodyne interferometry, which is an additional advantage over amplitude polarimetry.

This technique was first proposed by Dodel and Kunz [48] and independently by the diagnostic development group at Los Alamos [49]. The first experimental demonstration was published by Fulsuda, et al. [50], who reported the implementation of heterodyne polarimetry on a field-reversed configuration at Osaka University. Young [51] atternpted to implement a simultaneous measurement of Faraday rotation and density using, heterodyne techniques on Microtor at UCLA. Sensitivity problems were observed (at Faraday rotation levels well below the resolution obtained at Osaka University) that were not well understood. The Faraday rotation observed on adjacent chords had inconsistencies that prohibited the inferenc? of a current density profile. Gomez and Wolfe [52] proposed a heterodyne techrique that was tested on ALCATOR C. Work was not continued beyond the in:tial tests because of sensitivity problems that were observed.

This thesis research was done in parallel with these other efforts, with the objective of developing a heterodyne polarimeter/interferometer for poloidal magnetic field measurement on ZT-40M, a reversed-field pinch at Los Alamos National Laboratory. Some of the equipment development and assembly was done before this thesis research was initiated [49]. Without this effort, the final diagnostic development would not have been possible in the scope of a thesis. Further development was required in order to illow interferometric and polarimetric measurements to be 
made. The primary development involved the installation of a reference detector and the implementation of a four-signal data analysis technique (see Section 4.2.4) in order to eliminate the effects of imperfections of the rotating grating.

Significant sensitivity problems were observed with the polarimeter that were ultimately found to be caused by interaction between the probing laser beam and the tubulations that comprise the diagnostic access on ZT-40M. Reflectio.1s off the interna! metal walls of the tubulations caused polarization changes. Had the tubulations been stationary, this interaction would not have caused problems in the heterodyne polarimeter. However, because the tubulations vibrated when the machine fired, they introduced a time-varying birefringence, and induced "anomalous Faraday rotation." Installation of internally threaded inserts within the existing tubulations (to baffle the metallic reflections) suppressed the anomaly and allowed useful measurements to be made.

This effect may have caused the problems observed in the initial testing of the polarimeter on ALCATOR C, where the diagnostic access has exposed metal surfaces that vibrate when the machine fires. If even minute fractions of the beam are incident on metallic surfaces, significant polarization changes can take place.

While the primary task of developing a magnetic field diagnostic was in progress, preliminary measurements made with the FIR risstem contributed to the understanding of ZT-40M confinement physics. Sume of the contributions of the initial density measurements include the following:

- Off-axis density measured with the FIR system, when compared with the central chord measurements made by the two-color interferometer, indicated that the density profile was becoming flatter as the plasma current was increased.

- Edge-chord density measurements contributed to the determination of the inversion layer radius $c:$ sawtooth oscillations observed on ZT-40M.

- The FIR interferometer provided back-up measurements on ZT-40M during important data runs, and provided the primary density measurements when other interferometers were not operational.

Some of the interesting observations from the preliminary Faraday rotation measurements are listed below. 
- Observation of the amplitude changes of the carrier signal demonstrated that heterodyne polarimetry was essential for Faraday rotation measurements on ZT-40M.

- Faraday rotation enhancement was experimentally verified. Intentional misaligninent of optical components resulted in $\sim 1$ order of magnitude increase in the phase shift induced by the Faraday rotation on the carrier signal.

- The evolution of the Faraday rotation during plasma formation indicated that the initial breakdown position of the discharge changed with fill pressure, and that the current formed as an annular sheath that diffused inward on a $\sim 100 \mu \mathrm{sec}$ timescale.

- The Faraday rotation levels nicusured during high-current design-mode (or round-top) discharges agreed with expected levels based on independent density measurements and theoretical current profiles.

- The Faraday rotation level measured during high-current sustained (or flattop) discharges was much less than expected from theoretical profiles, indicating that the density and/or current profiles were broader than one would predict. The profiles became broader (or even flat) as the plasma current was increased, in stark contrast to the observations at the same plasma current levels for design-mode discharges. This suggests that the current scaling results obtained from the design-mode discharges (which were implemented to extend the scaling data to high current) are not representative of what can be achieved with sustained discharges.

- Fluctuations of the Faraday rotation caused by sawtooth oscillations, when compared with density measurements (made simultaneously), indicate that the current density profile peaks significantly during the sawtooth rise and flattens during the sawtooth crash. These observations agree with theoretical predictions [107] of the current density profile evolution during sawtooth oscillations.

Mosit of these observations require multichord measurements for the specific effects to be documented in detail. 


\subsection{Conclusions and Recornmendations}

As a result of this thesis research, the ability to measure Faraday rotation on a reversed-field pinch has been demonstrated. The problems that were encountered in the implementation of Faraciay rotation measurements on 2T-40M may be characteristic of any machine that has constrained diagnostic access, and may explain why the initial tests of Faraday rotation on ALCATCR $\mathrm{C}$ were unsuccessful. In any case, if Faraday rotation measurements are to be inplemented on a machine that has constraitied access, steps to eliminate reflection off metal surfaces must be taken. For machines that are yet to be built, and have plans for implementing farinfrared Faraday rotation measurements, specifications for the access requirements can be made before the machine is designed.

The capability of making polarimetric and interferometric measurements simultaneously with heterodyne phase-shift methods has also been demonstrated. As a result, the ability to make internal magnetic field measurements with a heterodyne polarimeter/interferometer is no longer in question. Multichord measurements could be made with an extension of this work, with a rather predictable commitment of inanpower and funds.

Given the potential information that could be obtained from internal magnetic field measurements, it is recommended that the extension to multichord operation proceed. The following paragraphs will outline briefly what needs to be done to achieve this capability.

With the development required to make Faraday rotation measurements on $2 \mathrm{~T}$ $40 \mathrm{M}$ completed, the polarirnetric sensitivity is now limited by laser power at the machine. The system that was used for single-chord measurements did not provide enough power to support multichord operation. The development that remains, before simultaneous density and magnetic field profiles can be measured on ZT$40 \mathrm{M}$, is the improvement of laser power at the machine. Work can then proceed with the design and assembly of the multichord hardware on $2 \mathrm{~T}-40 \mathrm{M}$.

This improvement can come from a direct increase in FIR laser power or by improving the efficiency of the components used to propagate the beam to and around the machine. Methods of improving the system to the point that multichord measurements are possible are itemized below. Some of the improvements are already in progress. 
Far-Infrared Laser The present laser produces $\leq 100 \mathrm{~mW}$ at $185 \mu \mathrm{m}$. Recent developments in FIR lasers have resulted in the capability of producing $\sim 400 \mathrm{~mW}$ at $185 \mu \mathrm{m}$ [108]. The primary improvement comes from increased $\mathrm{CO}_{2}$ power. Use of a two-laser system for heterodyning, rather than a rotating grating, could double the effective laser power at the machine, since a single laser beam would not have to be used to produce the two frequencies.

Waveguide Improvements in the waveguide would improve the laser power at the machine. A new waveguide has already been assembled on ZT-40M. It has a shorter pathlength (11 meters rather than 17), fewer $90 \%$ bends ( 3 rather than 7 ), and is made of less attenuating waveguide (1.5" precision-bore glass rather than 1" non-precision-bore plastic). These changes have improved the efficiency of the waveguide transmission from $5-10 \%$ to $\sim 50 \%$.

Polarizing Beam Splitters The 500 lp $i$ gold on mylar polarizing beam splitters are only $\sim 60 \%$ efficient in transmission. The polarizing beam splitters at the machine also need to be backed with TPX flats to suppress membrane-like vibrations, causing further losses (see Appendix $\mathrm{C}$ ). The heterodyne configuration (with the rotating grating) is only $\sim 25 \%$ efficient. The attenuation of the polarizing beam splitters accounts for most of the losses. If gold lines are coated directly on the TPX flats, and if a free-standing wire polarizer is used for the rotating grating configuration, large increases in effective laser power would result. A factor of $\sim 3$ could be gained at the rotating grating alone.

Miscellaneous Other improvements would contribute to the system performance in other ways. Schottky diode detectors have been installed on ZT-40M to avoid the high operating cost and degraded reliability of cryogenic detectors. The Schotiky diodes are not quite as sensitive as the GaAs photoconductors at $40 \mathrm{kHz}$; however the improvements attained above should help offset the reduced sensitivity. The Schottky diode sensitivity could be improved if a higher frequency were employed. This would also improve the time response of the measurements which presently are not able to track the fast density rise at plasma formation.

A new rotating grating, ruled completely around the circumference, was installed on the ZT-40M system. This should have eliminated the limit on the het- 
erodyne frequency imposed by the segmented grating (see Section 4.2.4). It was found that the new grating pattern was not as sharp as the old grating pattern, causing a decrease in reflectivity. In addition, mistakes in machining the grating cause segments of the wheel to be useless. As a result, the heterodyne frequency could only be increased to $\sim 100 \mathrm{kHz}$ - still not fast enough to track the density rise or take advantage of the frequency response of the Schottky diode detectors. Either a new grating wheel (fabricated with improved techniques [109]) or a twolaser heterodyning system should be considered.

The extension of the present work requires a significant investment of time and money. A new FIR laser may easily cost $\sim \$ 100,000$ (a new grating with improved efficiency may only cost $\sim \$ 10,000)$. The multichord optical components could easily cost $\sim \$ 25,000$. The system assembly and testing will take 3-5+ manyears (also a significant amount of money). The importance of the information that could be obtained, however, warrants the comriitment of manpower and funds. 


\section{Appendix A}

\section{Definitions in Toroidal Coordinates}

Although magnetic field measurements are of interest in all shapes of plasmas, most of the development and published measurements have been performed on toroidal plasmas. Therefore, toroidal coordinates are used throughout this work. The techniques described for making internal field measurements can also be applied to other plasmas; however, there is no generic or common coordinate system or notation, so confusion is generated unless one geometry is adhered to. The basic coordinate system for toroidal geometry is shown in Figure A.1. The unit vectors $\hat{r}, \hat{\theta}$ and $\hat{\phi}$ form a left-handed system, where $\hat{r}$ is the minor radial component, $\theta$ is the poloidal component, and $\phi$ is a toroidal component. For problems where toroidal effects are unimportant, and the plasma can be approximated as cylindricai, the angle $\theta$ becomes the cylindrical azimuth.

Another coordinate system often used is formed by the $\hat{R}, \hat{\phi}$, and $\hat{z}$ unit vectors, where these represent the major radial, toroidal, and vertical components, respectively. Often toroidal components are subscripted as $B_{T}$ rather than $B_{\phi}$ and $B_{p}$ rather than $B_{\theta}$, although this work attempts tc consistently use the $\phi$ and $\theta$ subscripts. Variations of this notation are sometimes found in the literature, but definitions are usually explicitly presented or the implications are obvious from context. Some of the terms encountered in descriptions of toroidal systems are defined below.

A fundamental quantity in toroidal magnetic fields describes the field line trajectory, relating the rate of change of the toroidal coordinate compared to the 


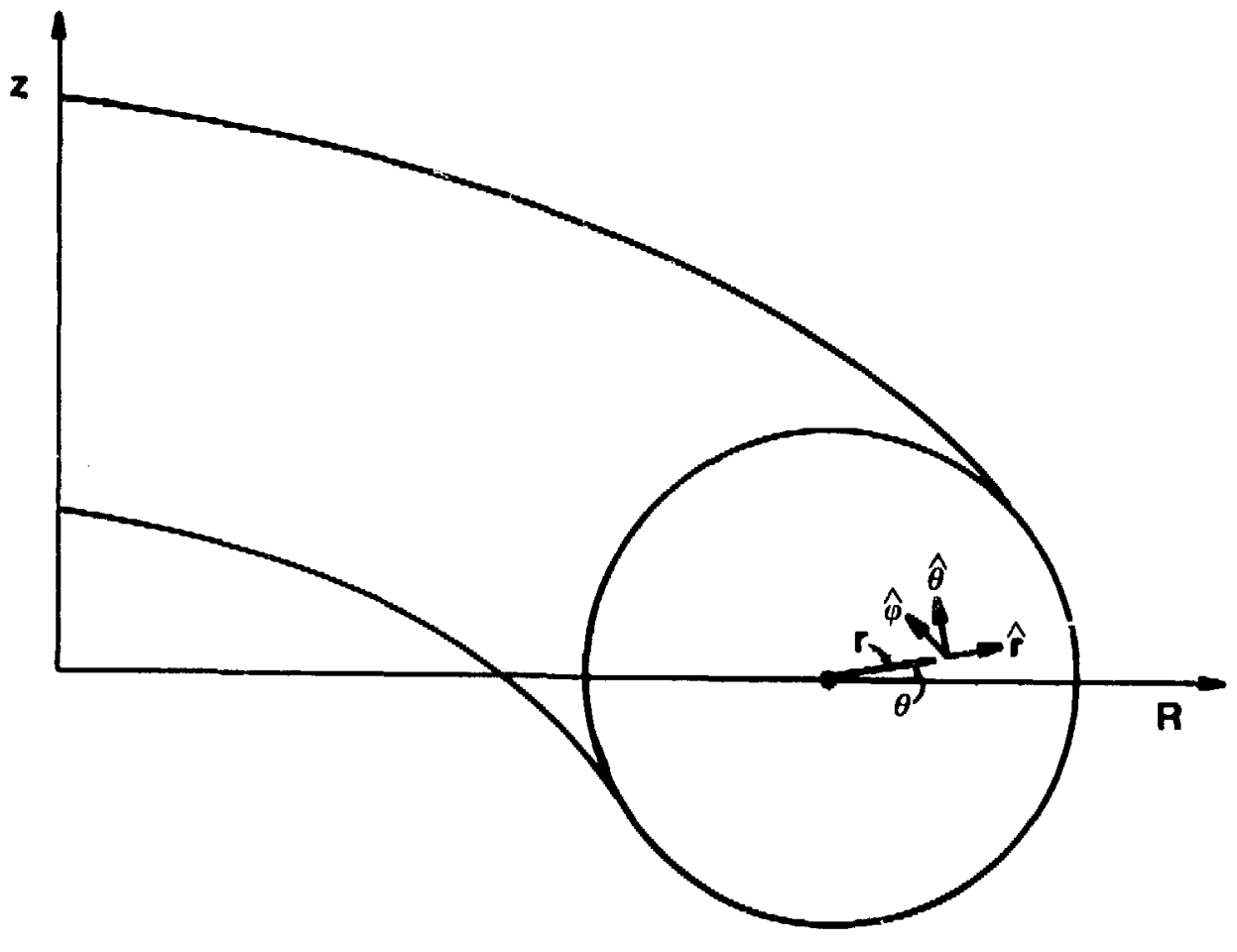

Figure A.1: Coordinate System for Toroidal Geometry 
poloidal component:

$$
\frac{d \phi}{d \theta}=\frac{r B_{\phi}(r)}{R B_{\theta}(r)} \equiv q(r)
$$

This ratio is commonly known as the "safety factor" because $q=1$ is found to be a stability threshold in tokamaks. Another quantity frequently found is the rotational transform,

$$
\iota(r)=\frac{2 \pi R B_{\theta}(r)}{r B_{\phi}(r)},
$$

which relates the change in poloidal angle a field line makes in a complete cycle in the toroidal direction.

The pitch of a field line is often referred to, and is given by:

$$
b=\frac{r B_{\phi}}{B_{\theta}}
$$

and the pitch length, the orthogonal analogue of the rotational transform, is:

$$
\ell_{p}=\frac{2 \pi r B_{\phi}}{B_{\theta}}
$$

which represents the toroidal distance required to make a complete poloidal cycle of the field line.

Another parameter that enters into stability criteria is the magnetic shear:

$$
s=\frac{d}{d r}\left(\frac{B_{\theta}}{r B_{\phi}}\right)=\frac{d}{d r}\left(\frac{1}{b}\right),
$$

which is related to the radial rate of change of the field line pitch as a function of minor radius -

Sometimes, knowledge of the toroidal current density profile is of interest. The current densi ty is related to the poloidal field by Ampere's law:

$$
B_{\theta}(r)=\frac{\mu_{o}}{r} \int_{c}^{r} j_{\phi}(r) r d r .
$$

Therefore, measurement of the poloidal field is equivalent to the measurement of the toroidal current density. Similar expressions can relate other currents and fields. 


\section{Appendix B}

\section{Mathematical Methods for}

\section{Calculating Polarization Effects}

on $E M$ W aves

\section{B.1 The Crystal Optic Analogy of a Magnetized Plasma}

The formalism for describing the effects of propagation through a magnetized plasma is taken from crystal optic theory. This choice is motivated by realizing that the plasma causes effects on the $E M$ wave that are more cammonly seen in work with crystal optics. Many mathematical techniques that were developed for the study of crystals can be applied directly to the case of a magnetized plasma. A very extensive and useful review of crystal optic theory is presented by Ramachandran and Ramaseshan [110].

Crystals of interest are often anisotropic. As a result, the effects imposed on the $E M$ wave depend on the relative orientation of the wave propagation vector and the crystalline axes. For any orientation, there is a set of two orthogonal wave polarizations that have a uniqua property in the crystal. Their state of polarization is independent of position along the pathlength, and the refore the waves propagate through the crystal with no change in polarization. These waves are called characteristic waves, because they are the characteristic vectors of Equation 2.14. A general polarization can be resolved into characteristic components as it propagates in the crystal, and the final polarization is found when the characteristic waves, which may propagate at different phase velocities, are recombined. 
To determine the characteristic polarizations, the characteristic value problem needs to be solved. Brandstatter $[54 \mid$ discusses the solution of this problem. An alternative approach is presented by Heald and Wharton [53] where one defines a wave polarization coefficient $E_{x} / E_{y}$. If this ratio is real, the characteristic wave is linearly polarized. If it is complex, the characteristic wave polarization is elliptical. For the special case where $E_{x} / E_{y}= \pm i$, the characteristic waves have left- and right-hand circular polarizations, respectively. For polarizations with an electrostatic component, a longitudinal polarizatior coefficient, $E_{2} / E_{x}$, can be used to describe the state of polarization $\left(E_{\mathrm{z}} / E_{\mathrm{v}}\right.$ can also be used). Again, if this coefficient is real, the characteristic wave is linearly polarized; if it is imaginary, it is elliptically polarized.

Polarization changes can only result if the medium exhibits birefringence or dichroism. Birefringence refers to the case when the two characteristic waves travel at different phase velocities in the medium. Polarization changes occur when the final relative phase between characteristic polarizations differs from the initial relative phase. Dichroism refers to the selective attenuation of one wave over the other. In this case, polarization changes take place because the ratio of amplitudes of the final wave differs from the ratio of the initial waves. Birefringence and dichroism are classified by the types of polarization that are characteristic. A medium with characteristic waves that are circularly polarized it is termed circularly dichroismatic or circularly birefringent (often called optically active). For linearly polarized characteristic waves the medium is said to exhibit linear birefringence or dichroism, although the adjective "linear" is commonly dropped in this case. If a medium is described as birefringent, it is assumed that it exhibitc linear birefringence. The general case is seen when the characteristic polarizations are elliptical, and the medium is said to be elliptically birefringent or dichroismatic

For a general problem in crystal optics (and magnetized plasmas), there is a systematic treatment of polarization effects that requires tlaat one:

1. determine the characteristic waves for the orientation of interest,

2. calculate the phase velocity (or related quantity) for these characteristic waves, and

3. calculate the attenuation efiects for these waves. 


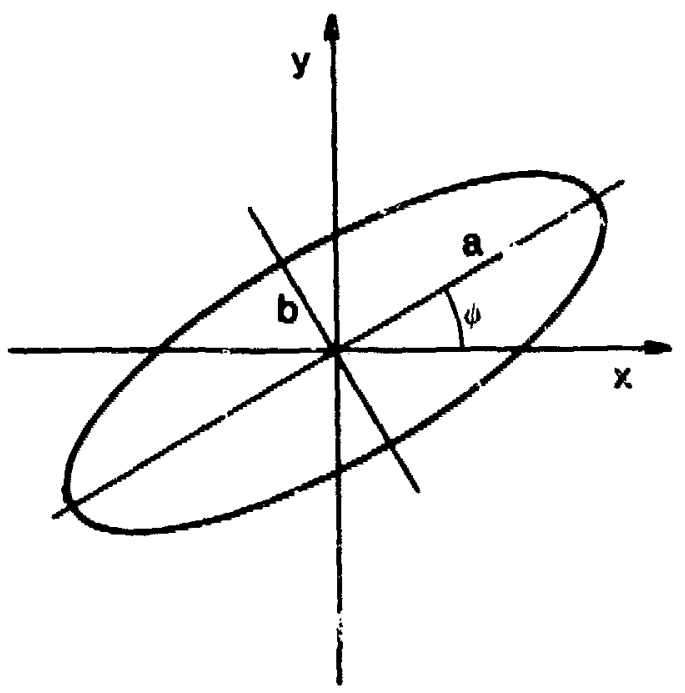

Figure B.1: Polarization Defined in Terms of Ellipticity and Azimuth

Use of these elements allows the determination of the final polarization state once the inpu: polarization conditions are known.

Dichroism in a plasma is a result of collisional effects, which are negliyible for the conditions of interest. The plasma can be taken as lossless for the radiation frequencies that are used for $E M$ wave probing of most plasratas. For highly collisional plasmas or low radiation frequencies, dichroism can have effects on the wave polarization. A threshold condition is defined and dichroismatic efiects are described by Vuolo and Galvão |111].

\section{B.2 Polarization Description and Evolution: The Poincaré Sphere}

It is conventiona? to describe the polariagtion of an EM wave in terms of its ollipticity and aximuth (e.g., [88]). Figure B.1 shows how these quantities are used to define the state of polarization of an $E M$ wave. The polarization refers to the locus of points that correspond to the tip of the electric field vector for a wave period. The eilipticity of the polarization is defined by the angle $\gamma$, where $\tan \gamma= \pm b / a$ for $b \leq a$. Negative values denote the case when the vector ratates in a clockwise sense when viewed by an observer looking towards the source. Positive values cor- 


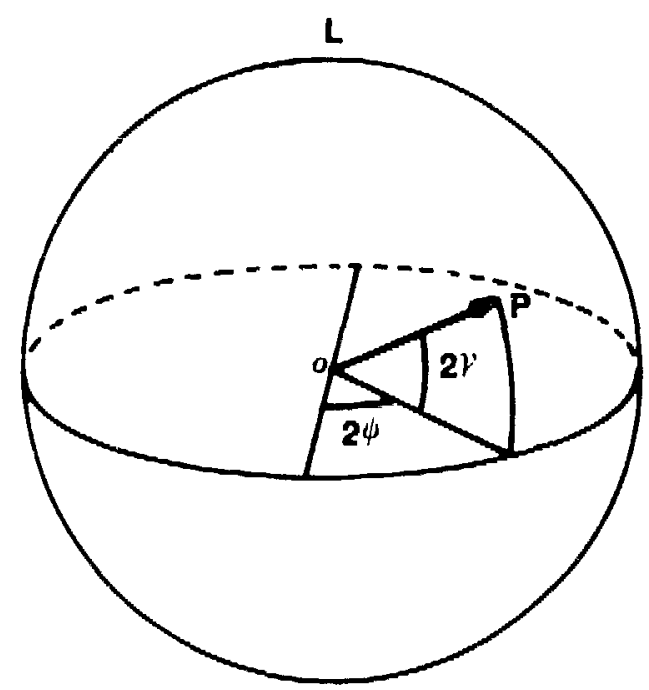

A

Figure B.2: Description of Polarization on Poincaré Sphere

respond to counter-clockwise vector rotation. The azimuth of the polarization is defined to be the angle between the major axis of the ellipse and some reference. In Figure B.1 the reference is chosen to be the $x$-axis. The state of polarization is uniquely defined when the azimuthal and elliptic angles are spezified.

This state of polarization ir terms of the angles and can conveniently be represented as a point on the surface of a unit sphere, called the Poincaré sphere, after H. Poincaré $[112]$, who first suggested this representation. An example of this representation is shown in Figure B.2. The north pole of the sphere represents a left-handed circular polarization and the south pole a right-handed circular polarization. The equator of the sphere is where linear polarizations are represented. A point on the sphere at longitude $2 \psi$ and latitude $2 \gamma$ represents a polarization with azimuth $\psi$ and ellipticity $\gamma$. Therefore, the state of polarization is also uniquely defined by a point on the surface of the Poincare sphere.

The real utility of the Poincare sphere is not in polarization representation, but in the simple operation required to determine polarization changes that result from propagation through an optical element. The characteristic polarizations of an optical element are directly opposed on a Poincaré sphere. The final polarization of the $E M$ wave is obtained from the initial polarization by rotating the sphere through an angle about the axis defined by the characteristic polarizations, where 


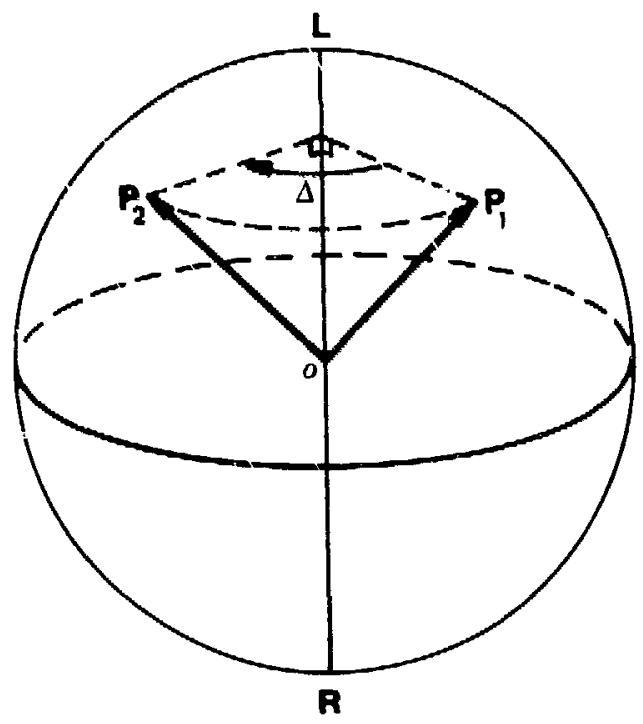

a) Polarization Evolution in a Ciroularly Birafringent Medium

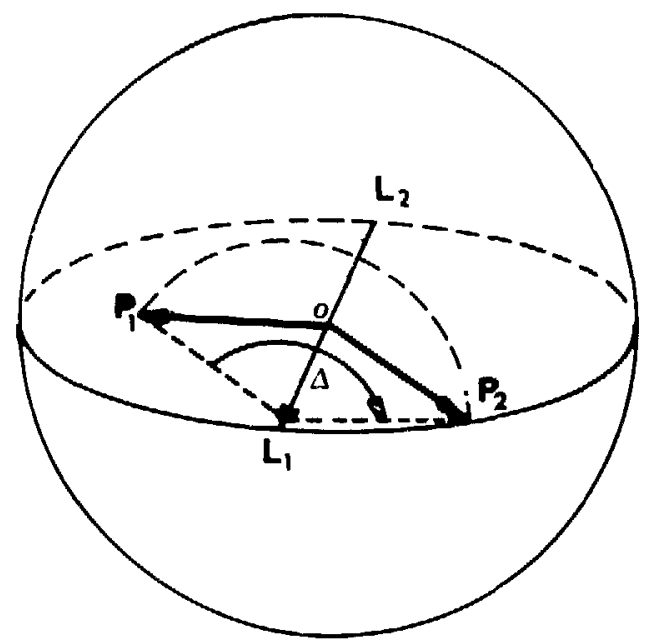

b) Polarization Evolution in a Linearly Birofringent Medium

Figure B.3: Operations Required to Determine Polarization Evolution 


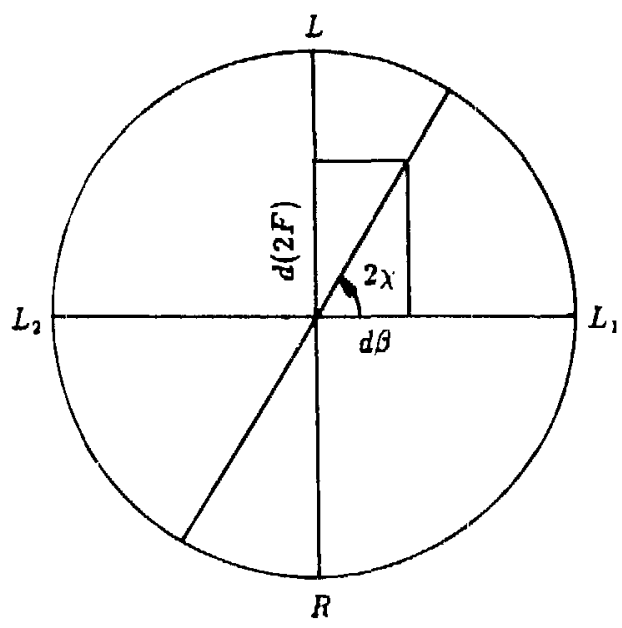

Figure B.4: Graphical Determination of Axis of Rotation for Elliptical Birefringence

where $\vec{\Theta}=(d \Delta / d z) \hat{c}_{f}$ with $\hat{c}_{f}$ being a unit vector in the direction of the fast characteristic polarization. In this way, the effect of linear, circular, or elliptical birefringence and iae resultant phase difference $\Delta$ between the orthogonal characteristic polarizations can be determined.

Sometimes use is made of Stokes parameters for descriptions of polarization effects. The zeroth Stokes parameter, $s_{0}$, represents the wave amplitude, and the other three parameters, $s_{1}-s_{3}$, are the cartesian coordinates of the point on the surface of the Poincare sphere that represents the polarization. The relation between the Poincaré representation and the Stokes representation is found in the following expression:

$$
\vec{P}=\left\{\begin{array}{c}
s_{1}=\cos 2 \gamma \cos 2 \psi \\
s_{2}=\cos 2 \gamma \sin 2 \psi \\
s_{3}=\sin 2 \gamma .
\end{array}\right.
$$

This notation facilitates the use of Eq. B.4 for studying polarization effects.

With the aid of the Poincaré representation, some of the optical elements used in many types of polarimeters can be better understood. Common optical elements, used because of their linearly birefringent characteristics, are called retardation plates. The two most frequently employed retardation plates are the $\lambda / 4$ plate and $\lambda / 2$ plate. Although they can be oriented in any direction, their function can be most clearly understood by placing them in a linearly polarized beam, with the 
exes at $\pm 45^{\circ}$ to the polarization. On the Poincaré sphere, the input polarization is represented as a point on the equator, at a position equally spaced between the points representing the characterictic polarizations, or the retardation plate axes.

A $\lambda / 4$ plate induces a phase difference of $\pi / 2$ between the characteristic polarizations; therefore, the function of a $\lambda / 4$ plate is seen by rotating the sphere by $\pi / 2$ about the characteristic axis. Depending on which plate axis is the fast axis, the output polarization will be either a right- or leit-handed circular polarization. At orientations other than $\pm 45^{\circ}$, a $\lambda / 4$ plate converts linear polarizations to elliptical. However, the most common application of a $\lambda / 4$ plate is to convert linear polarizations to circular, or circular polarizations to linear.

Similarly, the function of a $\lambda / 2$ plate is seen by rotating the sphere through an angle $\pi$, which restores a linear polarization, with the polarization azimuth rotated $90^{\circ}$. However, unlike the $\lambda / 4$ plate, the $\lambda / 2$ plate also retains linear polarizations at orientations other than $\pm 45^{\circ}$, but the azimuthal rotation is changed. This illustrates the most common application of a $\lambda / 2$ plate, where by adjusting the orientation of the axes relative to the input polarization, the azimuth of linear polarization can be rotated through any angle.

Although the Poincaré sphere and Stokes parameter representations are useful tools for visualizing and calculating the effects that a single optical element has on $E M$ wave polarization, for calculations of $E M$ wave propagation in more complicated systems these techniques are quite cumbersome. For such calculations, the analysis techniques to be presented in the next section prove to be more useful.

\section{B.3 Jones Matrix Calculus for $E M$ Wave Prop- agation}

R.C. .Jones developed a matrix calculus for describing the evolution of a transverse monochromatic $E M$ wave in an optical system $\{113\},\{114\},\{115\}$. This method is based on the idea that any elliptical vibration can be decomposed into two orthogonal linear vibrations with the appropriate amplitudes and phases. The general vibrations can then be represented completely by the columu vector:

$$
\vec{E}=\left(\begin{array}{c}
A_{1} \\
A_{2}
\end{array}\right)
$$


where $A_{1}$ and $A_{2}$ are the resolver components of the elliptical vibration, and are generally complex numbers. The wave intensity is given by:

$$
I=A_{1} A_{1}^{*}+A_{2} A_{2}^{*}
$$

and the ratio $A_{2} / A_{1}$ describes the polarization state (see Section B.1). This treatment assumes that the incident wave is completely polarized, and none of the components within the system depolarize the wave. Analysis of depolarizing systems requires the use of the more complicated Mueller matrices [114]. Jones matrix calculus allows the evolution of the electric field vector to be expressed as:

$$
\vec{E}=\overline{\bar{M}} \cdot \vec{E}_{\text {。 }}
$$

where $\dot{E}_{\alpha}$ is the input electric field vector, $\vec{E}$ is the final electric field vector, and $M$ is a matrix which represents a single optical element, or can be derived by multiplication of single element matrices to represent an entire optical system.

A useful representation of the more common matrices that apply to most optical systems is found in Reference [116]. Some of the matrices are presented here. The matrix for an element with linear birefringence $\beta$ is:

$$
\left(\begin{array}{cc}
\cos \beta / 2+i \cos 2 \theta \sin \beta / 2 & i \sin 2 \theta \sin \beta / 2 \\
i \sin 2 \theta \sin \beta / 2 & \cos \beta / 2-i \cos 2 \theta \sin \beta / 2
\end{array}\right)
$$

where $\theta$ is the azimuth of the fast axis (relative to the $x$-axis). The matrix for an element with circular birefringence $2 F$ is given by:

$$
\left(\begin{array}{cc}
\cos F & \pm \sin F \\
\mp \sin F & \cos F
\end{array}\right)
$$

where the upper signs are chosen if the right circular polarization is the fast characteristic polarization.

For an element with linear birefringence $\beta$ and circular birefringence $2 F$, Tabor and Chen $|117|$ give the following matrix:

$$
\left(\begin{array}{cc}
\cos \Delta / 2-i \cos (2 \chi) \sin \Delta / 2 & -\sin (2 \chi) \sin \Delta / 2 \\
\sin (2 \chi) \sin \Delta / 2 & \cos \Delta / 2-i \cos (2 \chi) \sin \Delta / 2
\end{array}\right)
$$

where, as before (see Eqs. B.2 and B.3), $\Delta \equiv\left(4 F^{2}+\beta\right)^{1 / 2}, 2 \chi \equiv \arctan (2 F / \beta)$. The matrix for a linear polarizer is:

$$
\frac{1}{\sqrt{1+\epsilon^{2}}}\left(\begin{array}{cc}
\cos ^{2} \alpha+\epsilon \sin ^{2} \alpha & (1-\epsilon) \sin \alpha \cos \alpha \\
(1-\epsilon) \sin \alpha \cos \alpha & \sin ^{2} \alpha+\epsilon \cos ^{2} \alpha
\end{array}\right)
$$


where $\alpha$ is the azimuth of the direction of transmission, and $\epsilon$ is the extinction ratio of the polarizer. For arbitrary orientation of these components in a cartesiun coordinate system, pre- and post-multiplication by a general rotation matrix is often useful. These matrices are applied as follows:

$$
\left(\begin{array}{cc}
\cos R & -\sin R \\
\sin R & \cos R
\end{array}\right)\left(\begin{array}{c}
\text { component } \\
\text { matrix }
\end{array}\right)\left(\begin{array}{cc}
\cos R & \sin R \\
-\sin R & \cos R
\end{array}\right)
$$

where $R$ is the rotation angle.

The Poincaré sphere and Jones matrix notations are equivalent for describing $E M$ wave propagation. As mentioned earlier, the Poincaré sphere is very useful for calculating and visualizing the polarization evolution of an EM wave as it propagates through a single optical element. The Jones matrix representation is useful for analyzing the effects of propagation through a more complicated optical system without the advantage of physical insight. With these mathematical tools in hand, the limiting cases of Appleton's equation can be analyzed in detail to determine what effects or plasma parameters can be measured by $E M$ wave probing. 


\section{Appendix C}

\section{Description of Far-Infrared Optical Components}

In this appendix, various optical components that have been used in the FIR polarimeter/interferometer will be described. One of the disadvantages of working in the far-infrared region of the electromagnetic spectrum is the lack of refined, commercially available optics. One reason for this problem is that FIR technology is quite new compared to optical, infrared, and microwave technology. Reasonably high-power radiation sources have only recently become reliable. Far-infrared sources are still quite weak compared to infrared and microwave sources, so the applications of FIR technology may still be limited in yearsi to come. As a result, there is not enough of a market for FIR components, and one is often forced to work with home-made components.

Techniques that are used in the far-infrared are often extensions of optical techniques to ionger wavelengths or microwave techniques to shorter wavelengths. Optical techniques need to be modified because of the large beam divergence for FIR radiation. Microwave techniques are sometimes limited because the physical scale of some components gets impractically small.

A description of the more commonly used FIR components will follow. In addition to a physical description of the components, some comments about the important practical details in the component application and operation will be given.

Retardation Plates The retardation plates are made of c-cut crystalline quartz. The thicknesses of the plates are based on optical constants reported by Lowenstein, 
et al.[72], which, for $185 \mu \mathrm{m}$ radiation, are $n_{o}=2.117, n_{\mathrm{e}}=2.159$. Unavcidably, there is also a differential abscrption, with $\alpha_{o}=0.1 \mathrm{~cm}^{-1}, \alpha_{e}=0.5 \mathrm{~cm}^{-1}$. The quarter wave plates are 1.5 inches in diarneter and 0.0433 inches thick. The half wave plates are 1.5 inches in diameter and 0.0866 inches thick.

Lenses Lenses used on the FIR system are made of TPX plastic [118|. TPX has the advantage of low absorption at boih FIR and visible wavelengths, which facilitates alignment. They are available commercially from various sources, but lenses designed for a specific application may be difficult to find. It was found that machining techniques could be refined to the point that lenses could be home-made. To make long focal-length lenses, machining the lens on a mill was preferable to a lathe. By tilting the spindle of the mill, and using a fy-cutter, the lens could be cut by positioning the material so the fly-cutter passed over the center, and the surface was cut by rotating the lens. By tilting the spindle further, sinaller radius surfaces could be formed. The machining marks that result are radial rather than circular, avoiding any Fresnel effects that might otherwise occur. The final finish was applied by polishing with a mild abrasive.

Polarizing Beam Splitters The polarizing beam splitters that are used in the system are 500 line-per-inch gold wires deposited on a thin mylar sheet. ${ }^{1}$ They are $\sim 60 \%$ transmitting for the peak polarization. They are $\sim 95 \%$ reflecting for the orthogonal polarization. They are 30:1 polarizers in transmission, but only $6: 1$ in reflection. The poor polarization upon reflection is a result of reflection off the mylar sheet (which contributes to transmissive losses). The beam splitters are typically mounted by stretching the material over a frame and bonding them to the frame with epoxy. For the beam splitters that were used in the MachZehnder configuration around the machine, thics mounts were found to vibrate when the machine fired, introducing errors in the interferometric measurements. The vibrations were suppressed by backing the beam splitter with a TPX flat. This increased the attenuation in transmission as well as the reflection of the wrong polarization, but eliminated the vibration problem. For use in transmission, simple pellicle mount vibrations do not adversely affect the beam.

\footnotetext{
${ }^{1}$ available from Buckbee-Mears, St. Paul, Minnesota
} 
Polarization analyzers are made of the same material as polarizing beam splitters, but are oriented differently for that application.

Non-polarizing Beam Splitters The non-polarizing beam splitters that were used deperided on the reflectivity required. The bean splitter used to reflect a small portion of the beam to the reference detector just after the rotating grating is $\sim 10 \%$ reflecting mylar film. TPX could also be used as a weak beam splitter when a more rigid optical component is required.

For applications where higher reflectivities are needed, inductive mesh beam splitters 119| are used. ${ }^{2}$ A wide variety of wire spacing and material are available, depending on the wavelength and reflectivity of interest. These meshes also have vibration problems in simple pellicle mounts. Therefore, backing the mesh with a TPX flat is required for these beam splitters as well.

It was found that both the dielectric and inductive mesh beam splitters were not perfectly non-polarizing when mounted at a $45^{\circ}$ angle of incidence. For dielectrics, it is a result of differences between $s$ and $p$ reflectivity, and for the inductive grids, it is because of a difference in the effective spacing seen by $s$ and $p$ components. The only location where this effect could be important is within the Mach-Zehnder contiguration (sce Section 6.3), where extinction-ratio errors could result. It was found that the polarizing effects of the inductive grids could be minimized by orienting them properiy. If the inductive grid (oriented at a $45^{\circ}$ angle of incidence to produce a $90^{\circ}$ bend) is rotated within the mount, there will be positions where the projection of the square pattern appears rectangular, and positions where the projection of the pattern is diamond-shaped. It was found that the diamond-shaped patterns exhibited much weaker polarizing effects than the rectangular. With the diamond-shaped orientation, the polarization effects were acceptably weak.

\footnotetext{
${ }^{2}$ Also available from Buckbee-Mears, St. Paul, Minnesola.
} 


\section{Bibliography}

(1) N. J. Peacock. Measurement of magnetic fields in : roidal systems. In Sindoni and Wharton, editors, International Schoo ", Plasma Physics, pages 367-402, Euratom, Brussels, 1978.

(2) D. G. Muir and P. G. Carolan. Faraday rotation applied to a reverse field pinch. Journal de Physique, 7(40):833-834, 1979.

3] K. M. Ling and D. A. Baker. Analytic Solutions of $\nabla \times \vec{B}=\alpha \vec{B}$ in a Straight Cylinder with $\alpha=\alpha(r)$. Technical Report LA-10345-MS, Los Alamos National Laboratory, June 1985.

14) R. Gerwin. A Method of Inferring the Magnetic Field Profiles in the Reversed-Field Pinch. Technical Report LA-8738-MS, Los Alamos Scientific Laboratory, March 1981.

(5) Équipe TFR. Tokamak plasma diagnostics. Nuclear Fusion, 18(5):647-731, 1978.

6] J. B. McEride et al. Current penetration in tokamak-type discharges. $N u$ clear Fusion, 15:393-405, 1975.

17| H. Knoepfel and D. A. Spong. Runaway electrons in toroidal discharges. Nuclear Fusion, '9(6):785-829, 1979.

18 D. A. Baker et al. Experimental and theoretical studies of the ZT-40M reversed-field pinch. In Plasma Physics and Controlled Nuclear Fusion Research, 1984, Volume 2, pages 439-447, International Atomic Energy Agency, Vienna, 1985.

[9] A. Sykes and J. A. Wesson. Field reversal in pinches. In Eighth European Conference on Controlled Fusion, Prague, 1977. 
[10] R. W. Moses. Physies of reversed-field pinch sustainment. In M. Q. Tran and R. I. Verbeek, editors, 1984 International Conference on Plasma Physics, pages 455-471, Lausanne, Switzerland, June 1984.

11] E. J. Caramana, R. A. Nebel, and D. D. Schnack. Nonlinear, singlehelicity magnetic reconnection in the reversed-field pinch. Physics of Fluids, 26(5):1305-1319, May 1983.

12| R. A. Nebel et al. Comparison of Zero-Dimensional and One-Dimensional Thermonuclaar Burn Computations for the Reversed-Field Pinch Reactor (RFPR). Technical Report LA-8185-MS, Los Alamos Scientific Laboratory, January 1980.

[13] J. P. Christiansen and K. V. Roberts. Evolution of the re"ersed-field pinch. Nuclear Fusion, 18(2):181-197, 1978.

14 R. H. Lovberg. Magnetic probes. In R. H. Huddlestone and S. L. Leonard, editors. Plasma Diagnostic Techniques, chapter 3, pages 69-112, Academic Press, 1965.

|15| I. H. Hutchinson. Magnetic probe investigation of the disruptive instibility in tokāmak LT-3. Physical Review Letters, 37(6):338-341, 1976.

16| L. A. Charlton, R. M. Wirland, and G. H. Nelson. Equilibrium modeling of ISX-B tokamak discharges. Physics of Fluids, 27(7):1738-1742, July 1984.

[17] J. L. Luxon and B. B. Brown. Magnetic analysis of non-circular cross-section tokiamaks. Nuclear Fusion, 22(6):813-821, 1982.

18 A. E. Costley and Équipe TFR. Measurements of cyclotron emission from high-density tokamak plasmas in TFR. F'hysical Review Letters, 38(25):14771480, June 1977.

[19 1. H. Hutchinson. The polarization of electron cyclotron emission from ALCATOR tokamak. Plasma Physics, 21:1043-1052, 1979.

[20] William W. Heidbrink. Tokamak Diagnostics Using Fusion Products. PhD thesis, Princeton University, June 1984. 
[21] R. Cano, I. Fidone, and J. C. Hosea. Poloidal field measurements in the ST tokamak by harmonic generation at the upper hybrid layer. Physics of Fluids, 18(9):1183-1186, September 1975.

[22] R. B. Howell and T. Cayton. Magnetoacoustic response of a plasma for determining magnetic feld profiles. Plasma Physics, 24(9):1051-1064, 1982.

(23) M. H. Brennan, B. L. Jessup, and I. R. Jones. An investigation of the composition of a hydrogen plasma using magnetcacoustic oscillations. Australiun Journal of Physics, 31:477-487, 1978.

[24] S. M. Mahajan, D. W. Rose, G. L. Chen, and R. D. Bengtson. A New Diagnostic for $B_{p}(r)$ and $q(r)$. Technical Report DOE/ET/53036-28, Depart ment of Energy, April 1981.

[25] F. C. Jobes. Heavy ion beam probing. In H. Eubank and E. Sindoni, editors, International School of Plasma Physics, pages 158-177, Varenna, 1975.

[26 S. Masamune, M. Fukno, and H. Nishihara. A proposal for neutral beam probing of plasma current profile. Jou. 14. 1 of the Physical Society of Japan, 50(7):2426-2432, July 1981.

[27| R.J. Goldston, E. Mazzucato, R.E. Slusher, and C.M. Surko. Experiments on the ATC tokamak. In Sixth IAEA Conference on Plasma Physics and Controlled Fusion Research, pages 371-383, International Atomi: Finergy Agency, Vienna, 1977.

[28] F. C. Jobes. A technique for measuring the poloidal field via a perpendicular neutral beam of $H_{2}^{\circ}$. In Second International Conference on High Temperature Plasme Diagnostics, Santa Fe, New Mexico, 1978.

29] P. R. Forman and W. Riesenfeld. Optical Mixing as a Plasma Diagnostic. Technical Report LA-8262-MS, Los Alamos National Laboratory, 1982.

[30] R. A. Stern. Harmonic generation and frequency mising at plasma resonance. Physical Review Letters, 14(14):538-540, April 1965.

[31] R. A. Stern, D. N. Hill, and N. Rynn. Direct ion-transport measurement by optical tagging. Physics Letters, 93A(3):127-130, January 1983. 
[32] F. Alladio and M. Martone. Thomson scattering measurements from a current carrying plasma and the determination of local current density in a tokamak (T.T.F.2). Physics Letters, 60A(1):39-4i, January 1977.

\%.

|33| J. Sheffield. Plasma Scattering of Electromagnetic Radiation. Academic Press, New York, 1975.

34 M. J. Forrest, P. G. Carolan, and N. J. Peacock. Measurement of magnetic fields in a tokamak using laser light scattering. Nature, 271:718-722, February 1978.

35 D. Wroblewski and H. W. Moos. Demonstration of Zeeman effect polarimetry as a magnetic field ineasurement method in a tokamak. Bulletin of the American Physical Society, 30(9):1569, 1985.

36 K. McCormick et al. Measurement of the current distribution in Asdex during lower hybrid current drive using the Li-Beam/Zeeman effect technique. In 12th European Conference on Conirolled Fusion and Plasma Physics, European Physical Society, 1985.

37: W. P. West. Spectroscopic measurement of magnetic fields in tokamaks. In Fourth APS Topical Conference on High Temperature Plasma Diagnostics, Boston, Massachusetts, 1982.

38 D. M. Thomas and W. P. West. The Zeeman $j(r)$ development program ai TEXT. Bulletin of the American Physical Society, 29:1190, 1984.

139 P. G. Weber and G. A. Wurden. Lithium heam Zeeman spectroscopy diagnostic development. Review of Scientific Instuments, 56(5):1070, May 1985.

:40| H. Soltwisch and Équipe T.F.R. Experimental test of far-infrared polarimetry for Faraday rotation measurements on the TFR 600 tokamak. Infrared Physics, 21:287-298, 1981.

[41 S. E. Segre. The measurement of poloidal magnetic field in a tokamak by the change of polarization of an electromagnetic wave. Plasma Fhysics, 20:295307,1978 . 
[42] W. Kunz and G. Dodel. On the measurement of poloidal field distributions in tokamaks by far-infrared polarimetry. Plasma Physics, 20:171-174, 1978.

[43] C. H. Ma, D. P. Hutchinson, P. A. Staats, and K. L. Vander Sluis. Measurements of electron density and plasma current distribution in tokamak plasma. Internationai Journal of Infrared and Millimeter Waves, 3(2):263-277, 1982.

[44] H. Soltwisch. Plasma position detection and control in the TEXTOR tokamak by means of a far-infrared interferometer/polarimeter. Nuclear Fusion, 23(12):1681-1685, 1983.

[45] D. K. Mansfield et al. Multichannel far-infrared interferometer/polarimeter for TFTR. Bulletin of the American Physical Society, 30(9):1519, 1985.

[46] D. P. Hutchinson, C. H. Ma, P. A. Staats, and K. L. Vander Sluis. Simultaneous measurement of line electron density and Faraday rotation in the ISX-B tokamak. Nuclea, Fusion, 21(12):1535-1540, 1981.

[47 T. R. Price. Current density measurements on the TEXT tokamak. Bulletin of the American Physical Society, 29(8):1395, 1984.

[48] G. Dodel and W. Kunz. A far-infrared polari-interferometer for simultaneous electron density and magnetic field measurements in plasmas. Infrared Physics, 18:773-776, 1978.

[49] M. D. Bausman, P. R. Forman, F. C. Jahoda, and R. W. Peterson. Far infrared Faraday rotation measurements on ZT-40. In Fourth International Conference on Infrared and Millimeter Waves, page 48, Institute of Electrical and Electronics Engineers, Miami Beach, December 1979.

[50] T. Fukuda, S. Goto, T. Ishimura, and H. Ito. A heterodyne interferometric polarimeter with $28 \mu \mathrm{m} \mathrm{H}_{2} \mathrm{O}$ laser for Faraday rotation measurement. International Journal of Infrared and Millimeter Waves, 5(7):1039-1052, 1984.

[51] P. E. Young. Multichannel Far-Infrared Interferometer/Polarimeter. PhD thesis, University of California Los Angeles, August 1984.

[52] C. Gomez and B. Lax. Faraday rotation in alcator tokamaks. Journal of Applied Physics, 52(11):6572-6575, November 1981. 
[53] M. A. Heald and C. B. Wharton. Plasma Diagnostics with Microwaves. Robert E. Krieger Publishing Company, Huntington, New York, second edition, 1978.

[54] J. J. Brandstatter. An Introduction to Waves, Rays and Radiation in Plasma Media. McGraw-Hill, New York, New York, 1963.

|55| Schiff. Quantum Mechanics. McGraw-Hill, New York, 1955.

56 W. M. Barr. Method for computing the radial distribution of emitters in a cylindrical source. Journal of the Optical Society of America, 52(8):885-888, August 1962.

[57| W. Magnus and F. Oberhettinger. Special Functions of Mathematical Physics. Chelsea Publishing Company, New York, 1949.

58) M. Deutsch and I. Beniaminy. Derivative-free inversion of Abel's integral rquat: Applied Physics Letters, 41(1):27-28, July 1982.

[59] Y. Yasutomo, K. Miyata, S. Himeno, T. Enoto, and Y. Ozawa. A new numerical method for asymmetrical A bel inversion. IEEE Transactions on Plasma Science, PS-9(1):18-21, March 1981.

60| N. G. Demas, C. H. Ma, D. P. Hutchinson, and P. A. Staats. Determination of spatial distribution of plasma electrons by multibeam fir interferometry. IEEE Transactions on Plasma Science, PS-13(1):41-44, February 1985.

61) D. G. Shirk and N. M. Hoffman. Monte Carlo Error Analysis in X-Ray Spectral Deconvolution. Technical Report LA-UR. 84-2906, Los Alamos National Laboratory, September 1984.

[62] D. A. Boyd. Effects of density inhomogeneities on reflectometry. Bulletin of the American Physical Society, 30(9):1420, October 1985.

[63] F. C. Jahoda and G. A. Sawyer. Optical refractivity of plasmas. In Lovberg and Griem, editors, Methods of Experimental Physics, Volume 9B, chapter 11, Academic Press, New York, New York, 1971. 
[64] D. Véron. Submillimeter interferometry of high-density plasmas. In K. J. Button, editor, Infrared and Millimeter Waves, Volume 2, Academic Press, London, 1979.

[65] A. D. Craig. The measurement of electron density and poloidal magnetic field in a tokamak by polarization changes in an EM wave. Plasma Physics, 18:777-790, 1976.

[66] S. E. Segre. On the polarimetric measurement of electron density and poloidal magnetic field in tokamaks. Il Nuovo Cimento, 66B(2):153-163, December 1981.

[67] W. Kunz. First measurement of poloidal-field induced Faraday rotation in a tokamak plasma. Nuclear Fusion, 18(12):1729-1732, 1978.

[68 C. J. Buchenauer and A. R. Jacobson. Quadrature interferometer for plasma density measurements. Review of Scientific Instruments, 48(7), July 1977.

[69| D. R. Baker and S. T. Lee. Dual laser interferometer for plasma density measurements on large tokamaks. Review of Siientific Instruments, 49:919922,1978 .

[70] D. K. Mansfield, L. C. Johnson, and A. Mendelsohn. A vibrationally compensated far infrared laser interferometer for plasma density measurements. International Journal of Infrared and Millimeter Waves, 1(4):631-640, 1980.

[71] R. M. Erickson, P. R. Forman, F. C. Jahoda, and J. R. Roberts. Polarimetric measurement of plasma polcidal magnetic field via heterodyne phase shift methods. IEEE Transactions on Plasma Science, PS-12(4):275-280, December 1984.

[72] E. V. Lowenstein, D. R. Smith, and R. L. Morgan. Optical constants of far infrared materials. 2: Crystalline solids. Applied Optics, 12(2):398-406, February 1973.

[73] J. Shmoys. Proposed diagnostic mettiod for cylindrical plasmas. Journal of Applied Physics, 32(4):689-695, April 1961. 
|74| M. A. Pollack. Molecular gas lasers. In R. J. Pressley, editor, Handbook of Laser 3 with Selected Data on Optical Technology, chapter 8, pages 298-349, The Chemical Rubber Company, Cleveland, Ohio, 1971.

(75] D. T. Hodges, F. B. Foote, and R. D. Reel. Efficient high-power opcration of the cw far-infrared waveguide laser. Applied Physics Letters, 29(10):662-664, November 1976.

176| D. T. Hodges and F. B. Foote. Design and Performance of Flowing-Gas Electrical-Discharge $C O_{2}$ Lasers. Technical Report SAMSO-TR-78-141, The Acrospace Corporation, El Segundo, California, Dacember 1978.

$\{77 \mid$ G. Busse, E. Basel, and A. Pfaller. Application of the opto-acoustic effect to the operation of optically pumped far-infrared gas lasers. Applied Physics, $12: 387-389,1977$.

;8| E. J. Danielewicz, T. A. Galantowicz, et al. High performance at new FIR wavelengths from optically pumped $\mathrm{CH}_{2} \mathrm{~F}_{2}$. Optics Letters, 4(9):280-282, September 1979.

79 D. J. E. Knight. Ordered list of far infrared laser lines (continuous, $\lambda>$ $12 \mu \mathrm{m})$. Technical Report Qu 45, National Physical Laboratory, Teddington, Middlesex, U. K., February 1981.

(80 T. Vogel and G. Dodel. Acousto-optic modulation in the far-infrared. Infrared Physics, 25:315-318, 1955.

181| P. R. Forman. Producing a Rotating Linear Polarization Electro-optically. Technical Report LAUR-85-XXXX, Los Alamos National Laboratory, 1985. To be published.

$82 \mid$ D. K. Mansfield and L. C. Iolnnson. A magneto-optically modulated $\mathrm{CH}_{3} \mathrm{OH}$ laser for Faraday rotation measurements in tokamaks. International Journal of Infrared and Millimeter Waves, 2(4):651-663, 1981.

[83 S. M. Wolfe, K. J. Button, J. Waldman, and D. R. Cohn. Modulated submillimeter laser interferometer system for plasma density measurements. Applied Optics, 15(11):2645-2648, November 1976. 
[84] T. Lehecka et al. Development of high power, twin frequency far-infrared lasers for fusion diagnostics. Review of Scientific Instruments, 56(5):940, May 1985.

[85] D. Véron. High sensitivity HCN laser interferometer for plasma electron density measurements. Optics Communications, 10(1):95-98, January 1974.

|86| R. W. Peterson, F. C. Jahoda, and D. A. Platts. Doppler shift of $1 \mathrm{MHz}$ at $119 \mu \mathrm{m}$. Bulletin of the American Physical Society, 23(7):773, September 1978.

[87| Francis A. Jenkins and Harvey E. White. Fundementals of Optics. McGrawHill, Inc., New York, fourth edition, 1976.

(88) Max Born and Emil Wolf. Principles of Optics. Pergamon Press, Oxford, England, sixth edition, 1980.

[89] E. A. J. Marcatili and R. A. Schmeltzer. Hollow metallic and dielectric waveguides for long distance optical transmission and lasers. The Bell Syaten。 Technical Journal, 43:1783-1809, July 1954.

[90| J. P. Crenn. A study of waveguides for far infrared interferometers measuring electron density of tokamak plasmas. IEEE Transactions on Microwave Theory and Techniques, MTT-27(6):573-577, April 1979.

(91) J. P. Crenn. Optical theory of Gaussian beam transmission through a hollow circular dielectric waveguide. Applied Optics, 21(24):4533-4541, December 1982.

992 ? G. E. Stillman et al. Far-infrared photoconductivity in high-purity epitaxial GaAs. Applied Physics Letters, 13(3):162-163, August 1968.

93) L W. Kunz and J. M. J. Madey. A fast sensitive GaAs photoconductor system with a cryogenic preamplifier. IEEE Transactions on Microwave Theory and Techniqucs, MTT-22(12):1118, December 1974.

[94 H. R. Fetterman, P. E. Tannenwald, B. J. Clifton, C. D. Parker, W. D. Fitzgerald, and N. R. Erickson. Far-ir heterodyne radiometric measurements 
with quasioptical Schottky diode mixers. Applied Physics Letters, 33(2):151154, July 1978.

195 D. P. Neikirk et al. Far-infrared imaging antenna arrays. Applied Physics Letters, 40(3):203-205, February 1978.

|96| D. B. Rutledge, D. P. Neikirk, and D. P. Kasilingam. Integrated-circuit antennas. In K. J. Button, editor, Infrared and Millimeter Waves, Voiume 10, Academic Press, London, 1983.

97 B. J. Clifton. Schottky-barrier diodes for submillimeter heterodyne detection. IEEE Transactions on Microwave Theory and Techniques, MTT-25(6):457463, June 1977.

98) K. A. Klare. One-trace phase-angle reconstruction. In Third Topical Conference on High Temperature Plasma Tiry.ostics, page 126, American Physical Society, March 1980. UCLA Report No. PPG 460.

99| The author is grateful for the efforts of Dr. P. R. Forman (LANL CTR-8) for providing the results of this straight-forward but tedious calculation.

[100] A. E. Siegman. An Introduction to Lasers and Masers. McGraw-Hill, New York, 1971.

101| J. D. Gaskill. Linear Systems, Fourier Transfı'ms, and Optics. John Wiley \& Sons, New York, 1978.

102. J. L. Monroe. Calculating beam propagation. Lasers \& Applications, 2:76 78, June 1983.

[103| These measurements werc provided by R. Bastasz of Sandia Livermorc.

104 C. J. Buchenauer. Acoustically Compensated Two-Wavelength Interferometry for Plasma Density Measurements: One-Dimensional Theory and Applications. Technical Report LA-9880-MS, Los Alamos National Laboratory, February 1984.

[105] J. A. Phillips et al. Observations of Ramped Current Operation in ZT-\{OM. Technical Report LA-10060-MS, Los Alamos National Laboratory, May 1984. 
[106] R. G. Watt and R. A. Nebel. Sawteeth, magneti. disturbances, and magnetic fiux generation in the reversed-field pinch. Physics of Fluids, 26(5):11681170, May 1983.

[107] K. A. Werley, R. A. Nebel, and G. A. Wurden. Transport description of the rise time of sawtooth oscillations in reversed-field pinches. Physics of Fluids, 28(5):1450-1453, May 1985.

[108] T. Lehecka et al. High power FIR lasers for diagnostic experiments. Bulletin of the American Physical Society, 30(9):1419, 1985.

\{109\} R. Howell and R. Erickson. Design and Fabrication of Rotating Diffraction Gratings for Far-Infrared Heterodyne Applirations. Technical Report LAUR-85-4216, Los Alamos National Laboratory, 1985.

[110] G. N. Ramachandran and S. Ramaseshan. Crystal optics. In S. Flügge, editor, Encyclopedia of Physics, Volume $X X V / 1$, Springer-Verlag, Berlin, 1961.

111) J. H. Vuolo and R. M. O. Galvão. Linear birefringence and optical activity in the far-infrared propagation in a tokamak. Plasma Physics, 25:1215-1235, 1983.

[112] H. Poincaré. Théorie Mathématique de la Lumière. Volume 2, G. Carré, Paris, 1892.

[113] R. C. Jones. A new calculus for the treatment of optical systems I. Description and discussion of the calculus. Journal of the Optical Society of America, 31:488-503, July 1941 .

[114] R. C. Jones. A new calculus for the treatment of optical systems V. A more general formulation, and description of another calculus. Journal of the Optical Society of America, 37(2):107-112, February 1947.

[115] R. C. Jones. A new calculus for the treatment of optical systems VII. Properties of the n-matrices. Journal of the Optical Society of America, 38(8):671 685, August 1948. 
(116) R. M. A. Azzam and N. M. Bashara. Ellipsometry and Polarized Light. North-Holland, Amsterdam, 1977.

117 W. J. Tabor and F. S. Chen. Electromagnetic propagation through materials possessing both Faraday rotation and birefringence: experiments with $\mathrm{yt}^{+}$erbium orthoferrite. Journal of Applied Physics, 40(7):2760-2765, June 1969.

118 G. W. Chantry et al. TPX, a new unaterial for optical components in the far infra-red spectral region. Infrared Physics, 9:31-33, 1969.

119| R. (.. McPhedran and D. Maystre. On the theory and solar application of inductive grids. Applied Physics, 14:1-20, 1977. 